\title{
Role for protein prenylation and "CAAX" processing in photoreceptor neurons
}

\author{
Nachiket D. Pendse
}

Follow this and additional works at: https://researchrepository.wvu.edu/etd

\section{Recommended Citation}

Pendse, Nachiket D., "Role for protein prenylation and "CAAX" processing in photoreceptor neurons" (2016). Graduate Theses, Dissertations, and Problem Reports. 6397.

https://researchrepository.wvu.edu/etd/6397

This Dissertation is protected by copyright and/or related rights. It has been brought to you by the The Research Repository @ WVU with permission from the rights-holder(s). You are free to use this Dissertation in any way that is permitted by the copyright and related rights legislation that applies to your use. For other uses you must obtain permission from the rights-holder(s) directly, unless additional rights are indicated by a Creative Commons license in the record and/ or on the work itself. This Dissertation has been accepted for inclusion in WVU Graduate Theses, Dissertations, and Problem Reports collection by an authorized administrator of The Research Repository @ WVU.

For more information, please contact researchrepository@mail.wvu.edu. 


\title{
Role for protein prenylation and " $C A A X$ ” processing in photoreceptor neurons
}

\author{
Nachiket D. Pendse \\ Dissertation submitted to the Department of Biology at \\ West Virginia University in partial fulfillment of \\ the requirements for the degree of \\ Doctor of Philosophy in \\ Biology
}
Visvanathan Ramamurthy, Ph.D., Chair
Maxim Sokolov, Ph.D.
Peter Mathers, Ph.D.
Andrew Dacks, Ph.D.
Shuo Wei, Ph.D.

\author{
Graduate Program in Biology \\ West Virginia University School of Medicine \\ Morgantown, West Virginia \\ 2016
}

Key Words: Prenylation, photoreceptor neurons, retina, phototransduction and vision

Copyright 2016

Nachiket Pendse 


\title{
ABSTRACT \\ Role for protein prenylation and " $C A A X$ ” processing in photoreceptor neurons
}

\author{
Nachiket D. Pendse
}

The efficient folding, assembly and trafficking of phototransduction proteins from site of synthesis in the inner segment (IS) to the site of action in the outer segment (OS) is crucial for photoreceptor cell survival and function. Defects in this process are known to cause blinding diseases in humans including retinitis pigmentosa (RP) and Leber's congenital amaurosis (LCA). The lack of a therapeutic approach for treatment of various retinal degenerations is likely due a knowledge gap in the mechanisms underlying the folding, assembly and transport of prenylated phototransduction proteins. Prenylation and " $C A A X$ " processing is thought to be involved not only in membrane anchorage of proteins but also in trafficking and regulating interactions between proteins. However, in-vivo experimental evidence scrutinizing the role of prenylation in retinal neurons is absent. Moreover, there are a growing number of discoveries linking prenylation defects with different retinal disorders, such as RP, LCA, rod and cone dystrophy, achromatopsia. The purpose of my dissertation is to understand the role of prenylation in biosynthesis, transport and function of key players of phototransduction cascade in photoreceptor neuron. To investigate the role of prenylation and methylation in photoreceptor neurons, we created mice models lacking prenyl transferases (chapter 2 and 3) and methyl transferases (chapter 4) in photoreceptor neurons. In Chapter 1 of this dissertation, we discuss the general significance of prenylation in photoreceptor neurons. We focused on the essential role of prenylation in the function and stability of a variety of prenylated proteins involved in phototransduction pathway. In Chapter 2, data is presented from the first animal model we generated that lacks prenylation in cone photoreceptor neurons. In the study, we demonstrated that the geranylgeranyl lipid anchors on cone PDE6 acts as a "molecular grip" to facilitate either the interaction between cone PDE6 and chaperone AIPL1 or assembly, a step needed for synthesis of functional PDE6 in cones. In chapter 3, we investigated defects in retina and in various phototransduction protein due to lack of prenylation in retina. Here we illustrate that lack of farnesylation affects the proper localization of rod transducin and also results in defective translocation kinetics. Our findings from this works also shows that single lipid anchor on PDE6 is sufficient for its assembly. Chapter 4, we discuss the potential role for ICMT mediated methyl esterification in photoreceptor morphogenesis and function. Our study demonstrates the in-vivo requirement of ICMT- mediated methylation of transducin $\gamma$ and cone PDE6 for their membrane anchorage highlighting the key role of ICMT in retinal neurons. Finally, in Chapter 5 we discuss the most significant and novel findings from our work and strategies to fill the gaps in knowledge that remain concerning the role of prenylation in photoreceptor cells. Overall, our findings highlight the intricate role of prenylation and methylation in photoreceptor neurons in vivo. 


\section{ACKNOWLEDGEMENTS}

This work would not be possible first and foremost without my advisor, Dr. Visvanathan Ramamurthy. I began fresh out of my second year of graduate school where my schedule was regular and decided for me daily. Dr. Ramamurthy helped me understand how to manage my time properly and realize that very little happens as expected, at least in a Ph.D., and how to plan accordingly. Our weekly meetings helped keep me on track toward larger goals by assessing the value of each experiment and properly planning it from the start. Taking ownership of projects is something that I learned from him. I must also acknowledge the help of Dr. Maxim Sokolov and Dr. Saravanan Kolandaivelu who provided much support and undivided attention whenever requested. Among my peers, I must first acknowledge my lab mates Zach Wright, Abi Hayes, Tanya Dilan, and Jesse Sundar. They really made grabbing a coffee much more enjoyable. I especially want to thank Dr. Nidhi Saraiya, Dr. Dan Murphy and Joe Murphy for their friendship and support both academically and personally.

I would not be here without the love and support of my family. I must thank my parents for always supporting me without question and advocating for whatever made me happy. Likewise, my siblings, Hrishi and Shona, have been a continuous source of support and relief from the day-to-day struggles of graduate school. A special thanks and my love goes to my greatest advocate, Devangi Patankar, who has been there every single day of this rewarding and often stressful experience. She has supported without repose and deserves all my gratitude. 


\section{TABLE OF CONTENTS}

CONTENTS

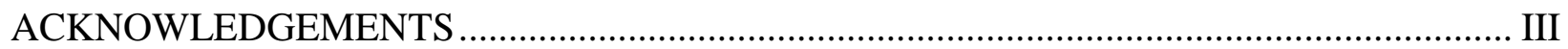

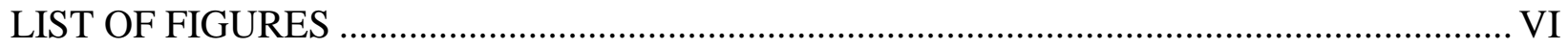

LIST OF ABBREVIATIONS ................................................................................ VIII

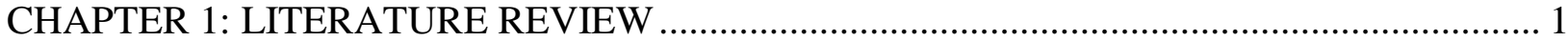

ISOPRENYL LIPIDS: MORE THAN JUST THE STICKY ANCHORS ..................................... 1

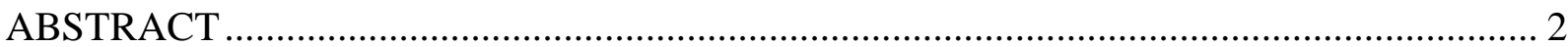

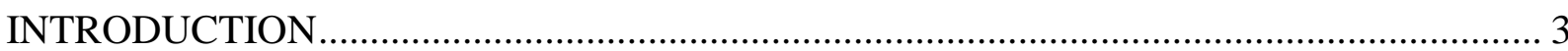

NEED OF PRENYLATION IN PHOTORECEPTOR NEURONS ………........................... 4

AN OVERVIEW OF PRENYLATION IN PHOTORECEPTOR NEURONS ……………... 5

PRENYLATED PROTEINS REQUIRE ADDITIONAL PROCESSING FOR

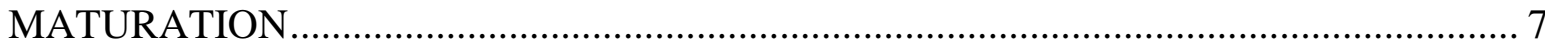

PHOTOTRANSDUCTION AND PRENYLATED PROTEINS IN PHOTORECEPTOR

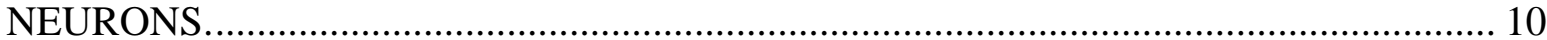

DEFECTS IN PRENYLATION LEAD TO RETINAL DEGENERATIVE DISEASES.... 19

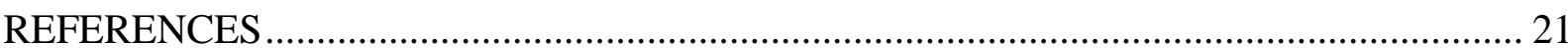

PGGT1B-MEDIATED PROTEIN GERANYLGERANYLATION IS CRUCIAL FOR ASSEMBLY OF PHOSPHODIESTERASE 6 AND FUNCTION OF CONE

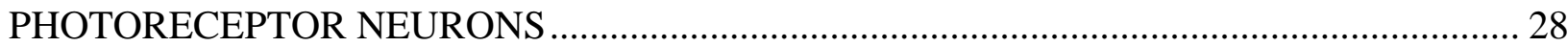

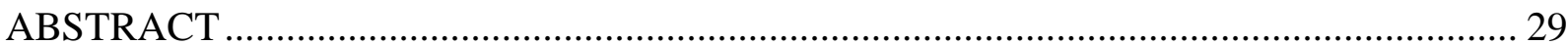

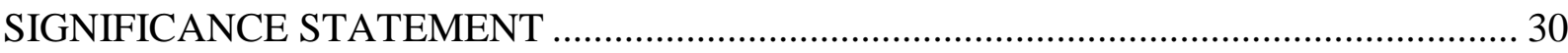

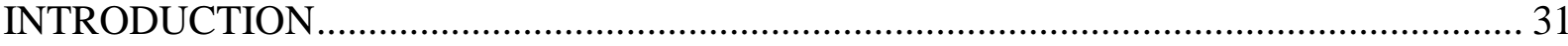

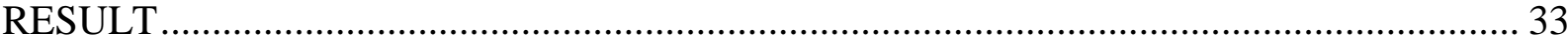

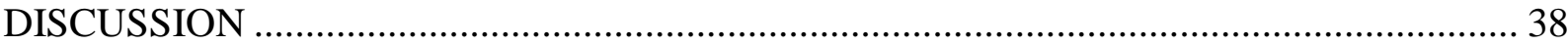

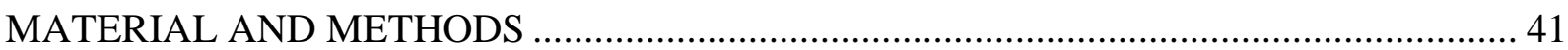

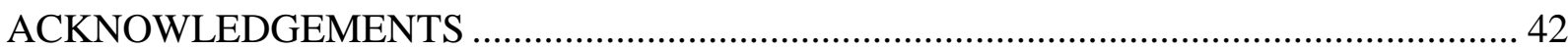

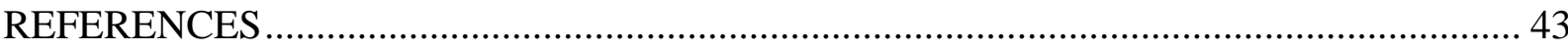

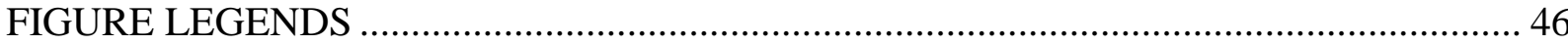

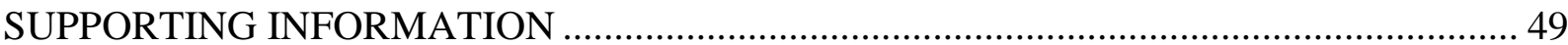

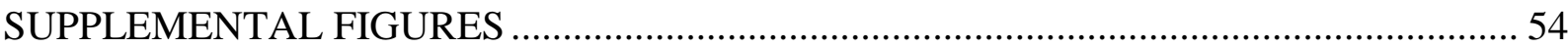

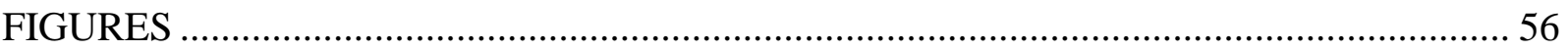

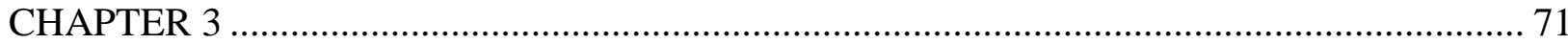


GENETIC ABLATION OF PRENYL TRANSFERASES LEADS TO LOSS OF PHOTORECEPTOR FUNCTION .............................................................................. 71

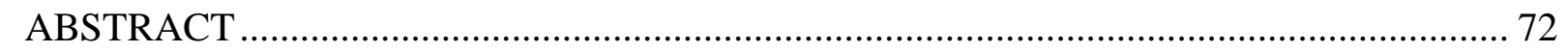

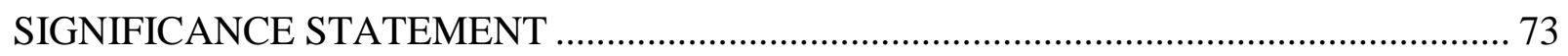

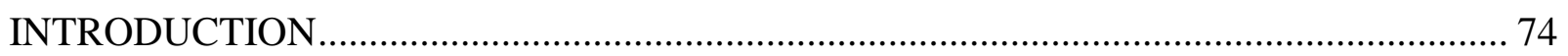

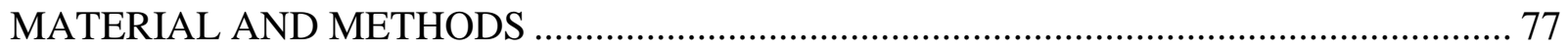

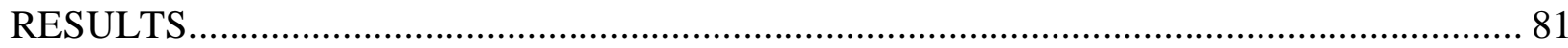

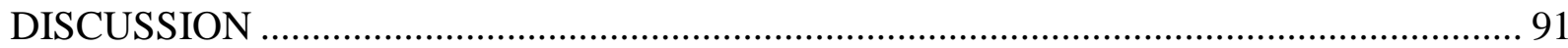

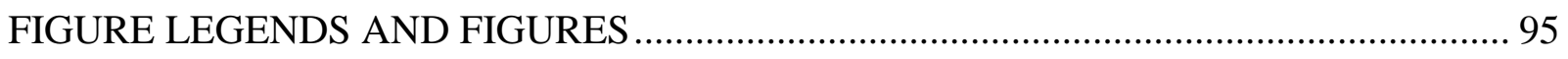

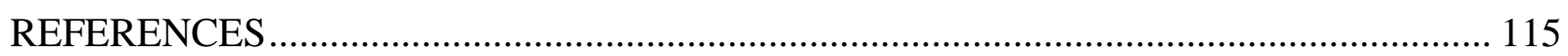

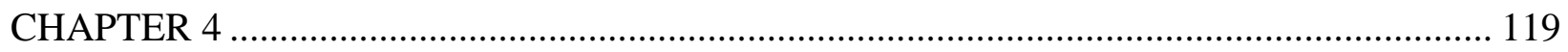

DEFICIENCY OF ISOPRENYLCYSTEINE CARBOXYL METHYLTRANSFERASE (ICMT)

LEADS TO PROGRESSIVE LOSS OF PHOTORECEPTOR FUNCTION........................... 119

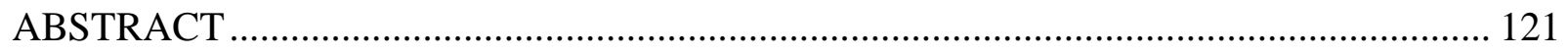

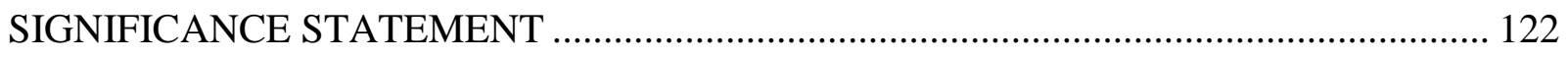

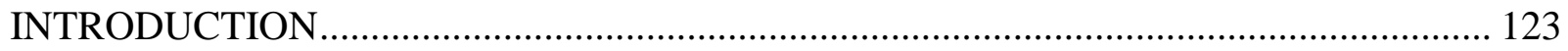

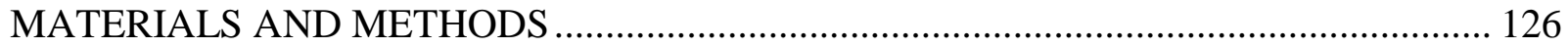

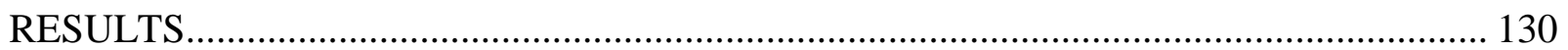

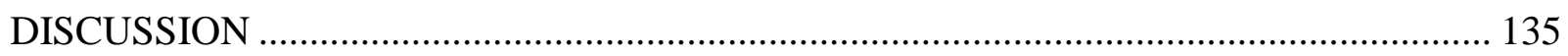

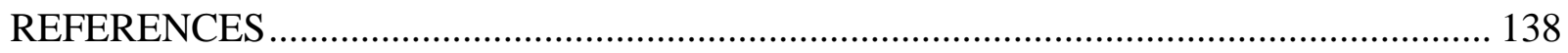

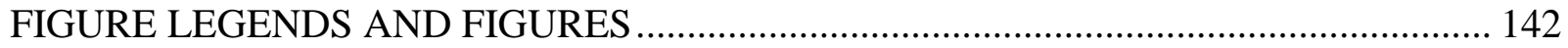

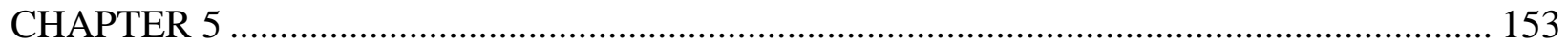

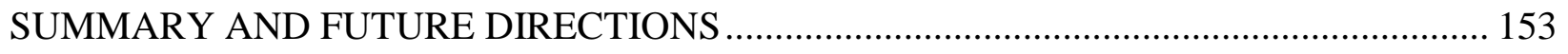

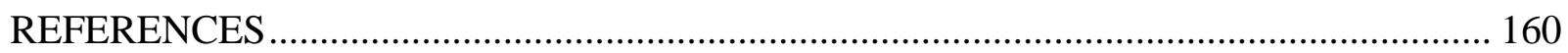




\section{LIST OF FIGURES}

\section{CHAPTER 1}

Figure. 1 Schematic representation of prenylation and postprenylation processing. ................... 7

Figure. 2 Rod and cone photoreceptor neurons are polarized cells. ......................................... 11

Figure. 3 Rod phototransduction cascade. ...................................................................... 12

\section{CHAPTER 2}

Fig. 1 Protein geranylgeranylation is crucial for cone photoreceptor function. .......................... 56

Fig. 2 Defective protein geranylgeranylation leads to reduction in the levels of phosphodiesterase and transducin in cone photoreceptors.

Fig. 3 Impaired membrane partitioning of cone phosphodiesterase and transducin in the absence

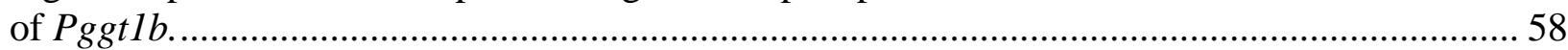

Fig. 4 Inefficient partitioning of cone phosphodiesterase and cone transducin- $\gamma$ subunit in Triton

X-114 detergent in the absence of Pggtlb. ......................................................................... 59

Fig. 5 Pggtlb-mediated protein geranylgeranylation is important for the assembly of cone

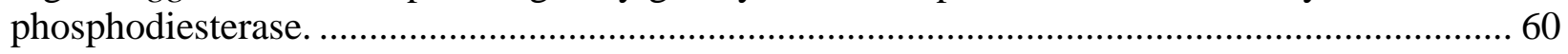

Fig. 6 Interaction of AIPL1 with cone phosphodiesterase requires protein geranylgeranylation.

Fig. 7 Rhodopsin kinase (GRK1) and Retinal guanylate cyclase (RetGC1) are absent in cones

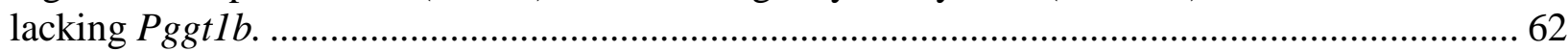

Fig. 8 Normal trafficking of phosphodiesterase to the cone OS in the absence of protein geranylgeranylation.

Fig. S 1 Generation of mice with cone-specific conditional removal of prenyl transferases. ...... 64 Fig. S 2 Progressive loss of photoreceptor function in mice lacking protein geranylgeranylation.

Fig. S 3 Rod photoreceptor function is unaltered in mice with cone-specific deletion of prenyl transferases. 65

Fig. S 4 Defective protein farnesylation does not affect the protein levels in cone photoreceptors.

Fig. S 5 Membrane association of cone-specific proteins does not require protein farnesylation. 67

Th. S 5 Me

Fig. S 6 Localization of GRK1 and cone PDE6 is unaffected in the absence of protein farnesylation.

Fig. S 7 Cone transducin localization is unaffected in the absence of protein geranylgeranylation.

\section{CHAPTER 3}

Figure. 1 Retinal morphology is unaltered in the absence of protein prenylation ......................... 96

Figure. 2 Light evoked ERGs are diminished in the absence of prenyl transferases..................... 98

Figure. 3 Photoreceptors degenerate progressively in absence of Pggtlb or Fntb .................... 100 Figure. 4 Lack of Pggtlb and Fntb in photoreceptors affects subset of phototransduction proteins 
Figure. 5 Membrane association of sub-set of prenylated impaired in the absence of prenyl

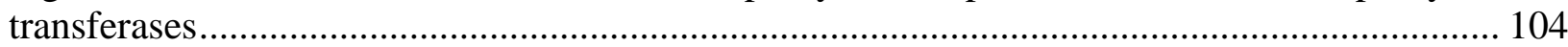

Figure. 6 Defective partitioning of phosphodiesterase and transducin- $\gamma$ subunit in Triton X-114

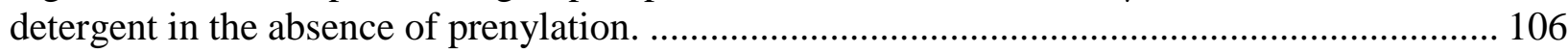

Figure. 7 Transducin is mis-localized in the IS in the absence of farnesylation ..................... 108

Figure. 8 Lack of Fntb affects the reappearance of translocated transducin to rod outer segments

Figure. 9 Levels of rod PDE6 and GRK-1 are severely affected in the absence of Pggtlb or Fntb

Figure. 10 Rod PDE6 assembly is not affected in the photoreceptors lacking Pggt $1 b$ and Fntb114

\section{CHAPTER 4}

Figure 1 Schematic representation of prenylation and postprenylation processing. ................ 146

Figure 2 Photoreceptor development is unaltered in the absence of Icmt expression. ............ 147

Figure 3 Visual deficit in mice lacking Icmt in the retina................................................ 148

Figure 4 Levels of subset of isoprenylated proteins are reduced in retinas lacking Icmt

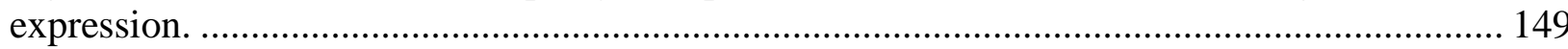

Figure 5 Membrane association of transducin and cone PDE6 is impaired in retinas lacking Icmt

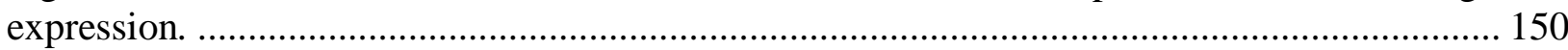

Figure 6 Rod PDE6 and transducin do not require ICMT-mediated protein methylation to be

transported to the OS

Figure 7 PDE6 and GRK1 levels in cones are reduced along with subset of other indicated

proteins in mice lacking ICMT-mediated protein methylation 


\section{LIST OF ABBREVIATIONS}

FTases-Farnesyl transferase

GGTase- Geranylgeranyl transferases

Fntb- Farnesyl transferase beta subunit

Pggt1b- Geranylgeranyl transferases beta subunit

PDE6 - Phosphodiesterase 6

PDE6 $\alpha$ - phosphodiesterase, alpha subunit

PDE6 $\alpha$ '- cone phosphodiesterase, alpha prime subunit

PDE6 $\beta$ - phosphodiesterase, beta subunit

PDE $\boldsymbol{\gamma}$ - phosphodiesterase, gamma subunit

GaT1- rod transducin, alpha subunit

G及1- rod transducin, beta subunit

G $\boldsymbol{\gamma}$ T1- rod transducin, gamma subunit

GaT2- cone transducin, alpha subunit

G及3- cone transducin, beta subunit

G $\boldsymbol{\gamma} \mathbf{T} 2$ - cone transducin, gamma subunit

GRK1 - Rhodopsin Kinase (G-protein receptor kinase 1)

RetGC-1- Retinal guanylate cyclase

PNA-Peanut agglutinin

DAPI - 4',6-diamidino-2-phenylindole

ERG - electroretinogram

OS - outer segment

ROS - rod outer segment

COS - cone outer segment

IS - inner segment

ONL - outer nuclear layer

INL - inner nuclear layer

IP - immunoprecipitation

CC - connecting cilium

RPE - retinal pigmented epithelium 


\section{CHAPTER 1: LITERATURE REVIEW}

\section{Isoprenyl Lipids: More than just the sticky anchors}

Nachiket D. Pendse ${ }^{1 \mathrm{a}}$ and Visvanathan Ramamurthy ${ }^{1 \mathrm{a}, \mathrm{b}, \mathrm{c} *}$

Departments of ${ }^{\mathrm{a}}$ Biology, and ${ }^{\mathrm{b}}$ Biochemistry, ${ }^{\mathrm{c}}$ Ophthalmology, ${ }^{1}$ West Virginia University, Morgantown, West Virginia 26506, USA;

*Address Correspondence to: Visvanathan Ramamurthy, West Virginia University Eye Institute, One Medical Drive, E-363, Morgantown, WV 26506-9193, Tel: 304598 6940; Fax: 304-5986928; Email: ramamurthyv@ wvumedicine.org

Key words: Prenylation and $-C A A X$ processing, photoreceptor neurons, phototransduction pathway, protein folding and assembly, retinal degenerative diseases.

Conflict of interests: The authors have no conflicts of interest 


\begin{abstract}
The photoreceptor cell sensory cilium is a complex organelle optimized for capturing light signal and transduction of that signal to downstream neurons. This specialized cilium requires a regulated series of interactions to produce the final functional outer segment with organized membrane discs loaded with phototransduction proteins. There is growing evidence that prenylation and $-C A A X$ processing play a major role in photoreceptor cell biology. Prenylation of proteins is considered as a static anchor needed only for retaining proteins in disc membranes. However, the extensive processing of prenylated proteins and reversibility of methylation (CAAX processing) suggests a critical role for lipid modification, not only in anchoring but also in multimeric subunit assembly, folding and regulation of interactions between proteins in the phototransduction pathway. Here we review the recent advances in our understanding of a dynamic role for prenylation and $C A A X$ processing in photoreceptor neurons. Prenyl transferases and number of their substrates have been implicated in retinal degenerative diseases and ciliopathies. The underlying mechanisms of retinal degeneration due to defects in the prenylated proteins will provide insights into pathological states that may guide therapeutic intervention.
\end{abstract}




\section{INTRODUCTION}

The molecular environment in which proteins interact with one another consists of two diverse settings; aqueous and membranous. One strategy that allows for protein-protein interactions in membranous settings is lipid posttranslational modification (PTM). In general, a lipid group is covalently attached to a protein at a consensus sequence to confer affinity to membrane domains or protein-interacting partners. Many proteins require PTM to function in several cellular processes. Examples of lipid PTMs are palmitoylation, myristoylation, glycosylphosphatidylinositol anchoring, and isoprenylation, which is the focus of this review. Protein isoprenylation is a lipid PTM that aids the interaction between the proteins and is thought to be needed for correct cellular localization of the protein. There are two types of protein isoprenylation, either a covalent attachment of a C15 lipid group (farnesyl) or a slightly longer C20 lipid group (geranylgeranyl group). Protein farnesylation and geranylgeranylation may provide lipid residues necessary to anchor the respective proteins to cell membranes or intracellular membranous structures. The attachment of prenyl groups occurs covalently through a thioether bond to one or two cysteines, the carboxyl-terminus of the protein.

It is believed that the prenylation of proteins is a static anchor needed only for retaining proteins in disc membranes. However, the extensive processing of prenylated proteins suggests a dynamic role for lipid modification, in biosynthesis, assembly and function of proteins in the phototransduction pathway. In this review, we highlight the crucial role of prenylation in the function and stability of a variety of prenylated proteins involved in phototransduction pathway in photoreceptor neurons. 


\section{Need of prenylation in photoreceptor neurons}

Retinal photoreceptor cells are highly compartmentalized membranous structures that are capable of detecting light and converting it into an electrical response via phototransduction. The unique structure that enables this remarkable process is a modified cilium with tightly packed membrane discs referred to as the outer segment (OS). The OS is critical for phototransduction because key signaling proteins are integrally and peripherally associated with the membrane. It is important to note that the machinery to synthesize these signaling proteins is present in a different cellular compartment, the inner segment (IS). Transport of proteins and membranes from IS to OS occurs along the connecting cilia (CC). The polarized nature of photoreceptor neurons requires a mechanism that can satisfy the extensive demand for efficient protein trafficking (1-4). On top of this, replacement of the entire OS and its contents occurs every 10 days due to phagocytosis by retinal pigment epithelial cells $(3,5)$. Thus the process of transport of proteins from IS to OS has to be rapid and continuous to populate the proteins in OS. For example, it is believed that about 80 rhodopsin molecules must be synthesized and delivered every second to replenish the OS (6).

Human disease may result from inefficiency in the process of protein trafficking in

photoreceptor cells. Notably, mutations of phosphodiesterase 6 (PDE6) or proteins necessary for its proper processing result in retinitis pigmentosa (RP) or Leber's congenital amaurosis (LCA), blinding diseases that are present at birth or during early childhood (7-9). Treatment for these diseases is scant and ineffective and this may be due to a knowledge gap in the mechanisms underlying the folding, assembly and transport of proteins to the site of action in the outer segment. Specifically, very little is known about the protein assembly and trafficking of PDE6. 
For PDE6, this lack of understanding is due to the inability to create a heterologous system to study it (10), which necessitates the limited and more time consuming use of animal models.

The high turnover rate of membrane discs as well as the protein machinery contained therein requires an efficient protein trafficking process. How are phototransduction proteins trafficked to OS? Rhodopsin is vectorially transported by rhodopsin transport carriers (RTC) $(1,11,12)$. On the other hand, transducin has been proposed to move by either vesicular transport or diffusion $(1,13)$. This leads to a pertinent question which is: How are proteins present in OS retained in disc membranes? One major contributor to protein retention in OS is prenylation of phototransduction proteins.

\section{An overview of prenylation in photoreceptor neurons}

Prenylation is the addition of a lipid, either a farnesyl or a geranylgeranyl group, to the Cterminal cysteine of proteins with a " $C A A X$ " motif, where $C$ stands for cysteine, $A$ for an aliphatic amino acid and $X$ for any amino acid. A farnesyl (C15) group is added by farnesyl transferase (FTase) enzyme, if the amino acid in the $X$ position is $S, L, Q, M, A, C, T$, or $H$ and a geranylgeranyl (C20) group is added by geranylgeranyl transferase (GGTase-I) enzyme, if $X$ is $L, F, I, V$, or $M(14,15)$. After prenylation of the "CAAX" proteins, the last three amino acid residues (-AAX) are cleaved by the protease RAS-converting enzyme 1 (RCE1) (16-20). Subsequently, isoprenylcysteine methyltransferase (ICMT) catalyzes the addition of a methyl group to the newly exposed isoprenylcysteine in endoplasmic reticulum (ER) membranes. The third prenyltransferase, called non-CAAX-prenyl transferase, Rab geranylgeranyl transferase or geranylgeranyl transferase-II (GGTase-II; used hereafter), recognizes Rab (member RAS oncogene family) proteins with a $C C, C X C, C C X, C C X X, C C X X X$ or $C X X X$ termination sequence $(15,21)$, where methyl esterification only occurs in the $C X C$ residues. In contrast to the 
$C A A X$-prenyl transferases, the heterodimer GGTase-II is unable to prenylate any peptides without the use of an accessory Rab Escort Protein (REP) as a substrate (22).

Addition of a prenyl group increases the hydrophobicity of proteins, enabling their interaction with membranes (23). Traditionally, prenyl groups have been thought to directly intercalate into the phospholipid bilayer, however the double-bonded nature of prenyl groups makes this interaction less likely (24). Another proposed role of prenylation is to aid in protein/protein interactions. Several examples of proteins with prenyl binding pockets exist including RhoGDI and $\operatorname{PrBP} \delta(12,25-30)$.

FTase and GGTase-I are heterodimeric enzymes with a common $\alpha$ - subunit coded by Fnta gene and unique catalytic $\beta$ - subunit, coded by Fntb gene in case of FTase and Pggtlb gene in case of GGTase-I. The $\alpha$ subunit recognizes and binds both substrates, farnesyl pyrophosphate (FPP) and geranylgeranyl pyrophosphate (GGPP). The $\beta$ - subunit catalyzes the transfer of farnesyl or geranylgeranyl group to C-terminal cysteine of prenylated proteins $(14,15,19,31,32)$. Germline knockouts of Fntb and Pggtlb are embryonically lethal indicating the importance of protein farnesylation and geranylgeranylation in normal embryonic development (33). Tissue specific removal of FTase or GGTase-I shows the importance of prenylation in liver (34). Altogether, these results emphasize the crucial role played by protein prenylation. However, the need for prenylation in neurons, where protein prenylation was discovered, is not known. Among neurons, photoreceptor cells contain multiple prenylated proteins, but the role for this lipid modification is not completely understood as yet.

The first prenylation defects that affect our vision were identified in persons with choroideremia (CHM), a condition with a defect in the gene that encodes a subunit of the (GGTase-II) protein complex, Rab escort protein-1 (REP-1) (22,35). In the retina, the cyclic 
GMP (cGMP) PDE6 $\alpha$ subunit, rod transducin $\gamma$ subunit, rhodopsin kinase and ceroid lipofuscins neuronal 3 proteins are substrates for FTases (36-40), whereas the cGMP PDE6 $\beta$ subunit, cone PDE6 and X-linked retinitis pigmentosa (RP) are substrates for GGTase-I $(38,41)$.

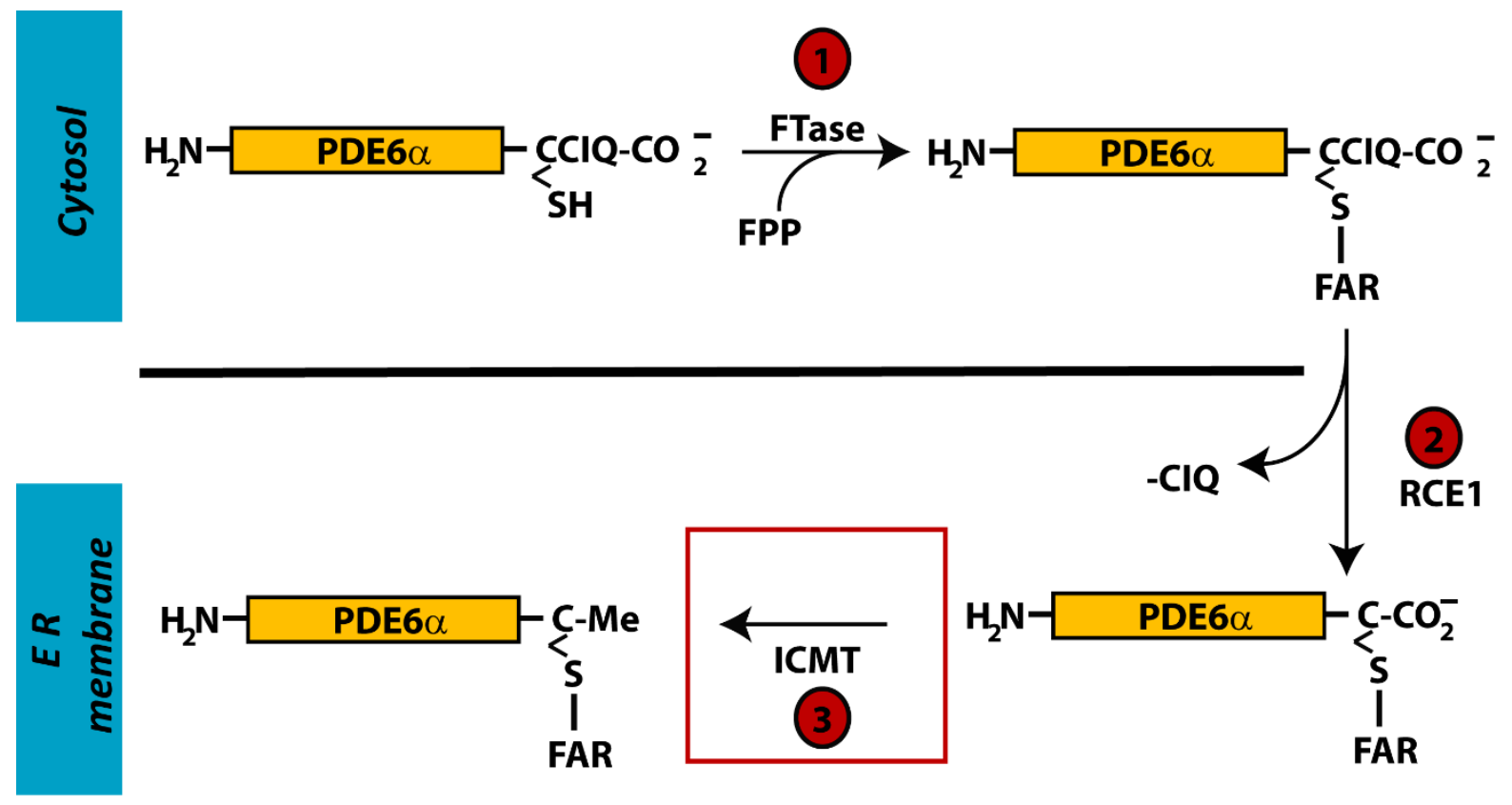

Figure. 1 Schematic representation of prenylation and postprenylation processing.

Rod PDE6 $\alpha$ is farnesylated. (1) Protein FTase-I in the cytosol adds a farnesyl lipid (FPP) to the cysteine of the $C A A X(C A A X=C C I Q$ for PDE6 $\alpha)$ motif. (2) RCE1-mediated endoproteolysis at the endoplasmic reticulum (ER) membrane, cleaves the last three amino acids of the $C C I Q$ motif (i.e., $-C I Q$ ). (3) ICMT, an ER membrane protein, catalyzes the methyl esterification of the farnesyl cysteine. After methylation, prenylated proteins are extracted from ER membranes by proteins such as PrBP $\delta$ and are further transported to photoreceptor OS (42).

Prenylated proteins require additional processing for maturation

Unlike other lipid modifications, prenylated proteins must undergo additional processing steps before becoming fully functional. After adding of the prenyl group, the last three amino acids are cleaved by the endoprotease RCE1. Subsequently, ICMT catalyzes the addition of a methyl group to the newly exposed isoprenylcysteine. " $C A A X$ " processing further increases the hydrophobicity of prenylated proteins and aids in their interaction with other proteins. For 
instance, in vitro liposome binding studies have shown farnesylated proteins exhibit 20 -fold increase in binding affinity when they are methyl-esterified (38).

\section{RCE1-mediated endoproteolysis of prenylated proteins}

After prenylation, proteins proceed to the ER for cleavage of the last three amino acids of the "CAAX" box by RCE1 (Fig. 1, Step 2). RCE1 is a multi-pass integral ER membrane zinc metalloproteinase that was identified in yeast in a sterile yeast mutant screen (43). The importance of RCE1 in mammals was established through genetic knockout of RCE1 which resulted in embryonic lethality (17). Embryos survived until embryonic day E15.5 without any gross morphological defects. Subsequent conditional knockout (CKO) studies revealed the importance of RCE1 in the heart and photoreceptor neurons, but liver and spleen CKO were unaffected $(18,44)$. The cause of lethality in the germline and heart knockout of Rcel has been suggested to be due to defects in Ras membrane association (44). Indeed, studies investigating the localization of K-Ras from germline knockout mice have shown that K-Ras is not localized at the plasma membrane (45). A requirement for $C A A X$ protein processing in retinal function was demonstrated by a study in which Rcel was inactivated in the neural retina (46). An absence of RCE1-mediated protein processing disrupted the transport of PDE6 to the outer segment (OS).

\section{ICMT-mediated methyl esterification is the final step of "CAAX" processing}

The final event of "CAAX" protein processing is methyl esterification of the newly exposed isoprenyl cysteine by ICMT (Fig. 1, Step 3) (47). ICMT catalyzes the addition of a methyl group utilizing S-adenosyl methionine (SAM) as the methyl donor. ICMT is a multi-pass integral ER membrane protein that is present as a dimer or higher order oligomer $(48,49)$. Experiments designed to identify methylated photoreceptor proteins uncovered a subset of rod 
OS proteins that incorporated a radioactive methyl group (50). The identity of the methylated proteins and their ability to incorporate a methyl group has been studied extensively, but the importance of this modification to photoreceptor function is not known $(37,39,51)$. Rod phosphodiesterase 6 (PDE6), the effector enzyme of the visual signal transduction cascade, was the first methylated protein to be identified in retinal lysates (50). The carboxyl terminus of PDE6 catalytic subunits terminates with a "CAAX motif," which triggers isoprenylation of the carboxyl-terminal cysteine (the " $C$ " of the $C A A X$ motif). PDE6 $\alpha$ and $\beta$ catalytic subunits are isoprenylated by farnesyl and geranylgeranyl lipids, respectively. Interestingly, rod PDE6 $\alpha$ incorporated a methyl group in an in vitro radioactive methylation assay, whereas PDE6 $\beta$ was not an efficient substrate for protein methylation (38). The methylation status of PDE6 $\alpha^{\prime}$, which is thought to be geranylgeranylated (41), is not known. Additional isoprenylated (farnesylated) photoreceptor proteins include the $\gamma$-subunit of rod transducin $(\mathrm{G} \gamma \mathrm{T} 1)$ and rhodopsin kinase (GRK1) (37,52).

Initially a milder phenotype was expected from an ICMT conditional knockout (CKO) mice than the RCE1 conditional knockout mice, because multiple methyltransferase enzymes were predicted. However, elimination of Icmt resulted in embryonic lethality of mice five days earlier than RCE1 CKO (53). To date, ICMT is the only protein known to catalyze the methyl esterification of " $C A A X$ " box proteins $(49,54)$. An implication of this result is that ICMT processes more proteins than RCE1 (53). Another likely cause of increased lethality is that lack of methylation exposes a carboxylate anion adjacent to the prenylated cysteine residue. A carboxylate anion in this particular position is thought to be lethal to cells based on "carboxylate anion positioning hypothesis" (53). Additional consequence of Icmt elimination is an increased rate of protein turnover (55). 
Methylation of isoprenylated cysteines is thought to increase the hydrophobicity of the protein and facilitate interactions with membranes (56). Also, in vitro approaches have shown that carboxyl methylation enhances certain protein-protein interactions. For example, methylation of $\mathrm{G} \gamma \mathrm{T} 1$ is thought to enhance the interaction of the transducin complex with metarhodopsin II (51). Furthermore, the interaction between C-terminal isoprenylated PDE6 peptides and prenyl binding protein $\delta(\operatorname{PrBP} \delta)$ is influenced by the methylation status of PDE6 (57).

Methylation has been proposed to be a dynamic modification but evidence for reversibility of this modification is still lacking. Early studies suggest that methylation of G $\gamma \mathrm{T} 1$ is reversible, but experiments purifying transducin from photoreceptors failed to identify a nonmethylated isoform $(51,58)$. We recently demonstrated that photoreceptors require carboxyl methylation of the C-terminus of a subset of their signal transduction proteins for function and survival (59), see chapter 4 of thesis. Our study demonstrates the in-vivo requirement of ICMTmediated methylation of rod T $\gamma$ and cone PDE6 for their membrane anchorage (59).

\section{Phototransduction and prenylated proteins in photoreceptor neurons.}

Proteins that play critical role in phototransduction (rhodopsin, transducin complex, cGMP phosphodiesterase 6 [PDE6], cyclic nucleotide-gated [CNGA1/3] channel subunits) and accessory proteins (guanylate cyclase [GC], GC-activating proteins or GCAPs and the GTPaseactivating protein [GAP] complex, rhodopsin kinase or GRK1, arrestin) are synthesized in the IS and must be transported through the $\mathrm{CC}$ to the OS. These proteins are either transmembrane (TM) proteins or peripherally associated membrane proteins that are attached to the membrane surface. Maintenance of the OS is energetically demanding due to continual signaling and the replacement of the entire OS and its contents every 10 days $(60,61)$. 


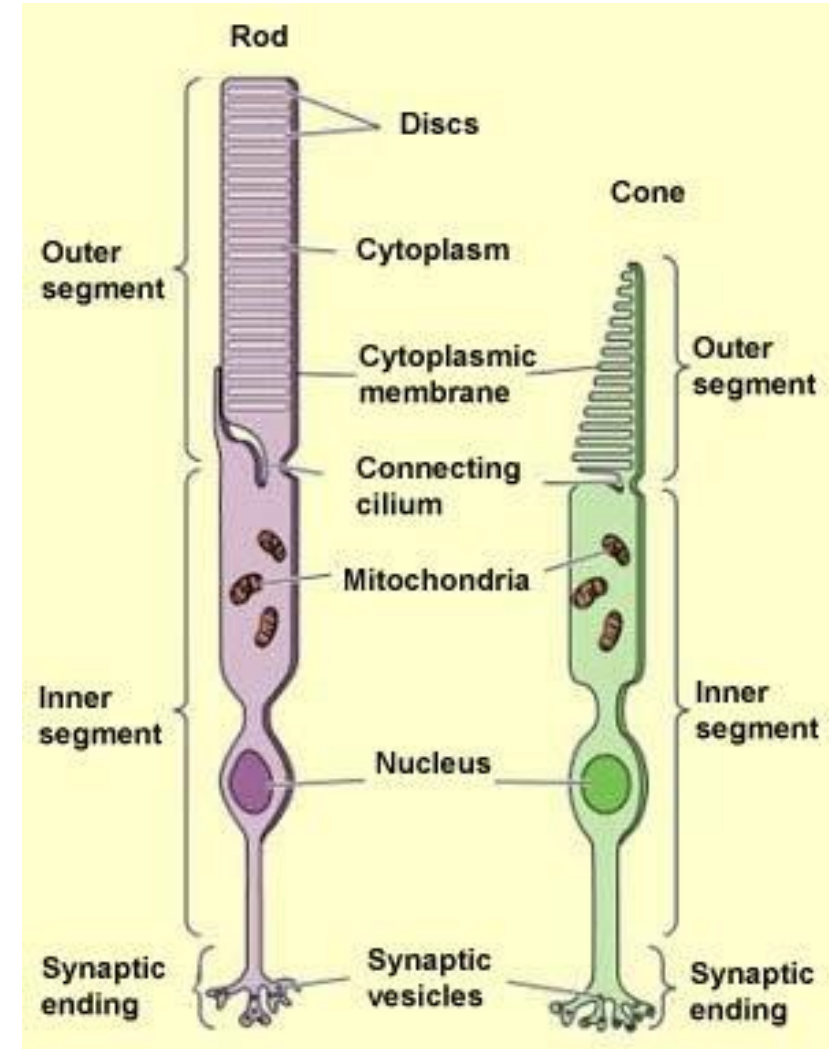

Figure. 2 Rod and cone photoreceptor neurons are polarized cells.

Photons are absorbed by rhodopsin in the disc membranes of the outer segment (OS). Phototransduction results in membrane hyperpolarization that is transmitted to bipolar cells at the synaptic terminal. Proteins are synthesized and processed in the inner segment (IS). Transport of proteins occurs along the connecting cilia (CC) at the transition zone. Retinal pigmented epithelium (RPE) cells phagocytose OS disc membranes. Nucleus is present in the cell body. Adapted from (62).

Two types of photoreceptors cells are present in the retina, rods to detect dim light and cones for color and bright light vision. Both cell types utilize similar signal transduction pathways, but the specific proteins and their post translational modifications differ between rod and cone cells. Vision begins in the OSs of rod and cone photoreceptors upon the absorption of photons by visual pigment molecules; the opsins (either rod or cone) become activated and start the phototransduction cascade. In general, the phototransduction pathway is initiated when a photon of light alters the conformation of an opsin molecule. Activated opsin exchanges GDP for GTP on the $\alpha$-subunit (GaT1) of heterotrimeric rod G-protein transducin (Fig. 3). GTP-bound 
$(\mathrm{G} \alpha \mathrm{T} 1)$ dissociates from rod $(\mathrm{G} \beta \gamma \mathrm{T} 1)$ and removes the inhibitory subunit from the membrane bound effector enzyme, phosphodiesterase 6 (PDE6)(63-65). PDE6 lowers intracellular concentrations of cGMP by hydrolysis. Reduction of cGMP closes cyclic nucleotide gated anion channels (CNGA), causing the photoreceptor cell to become hyperpolarized and initiate downstream signaling (66). Rhodopsin signaling is turned off by rhodopsin kinase (GRK1) mediated
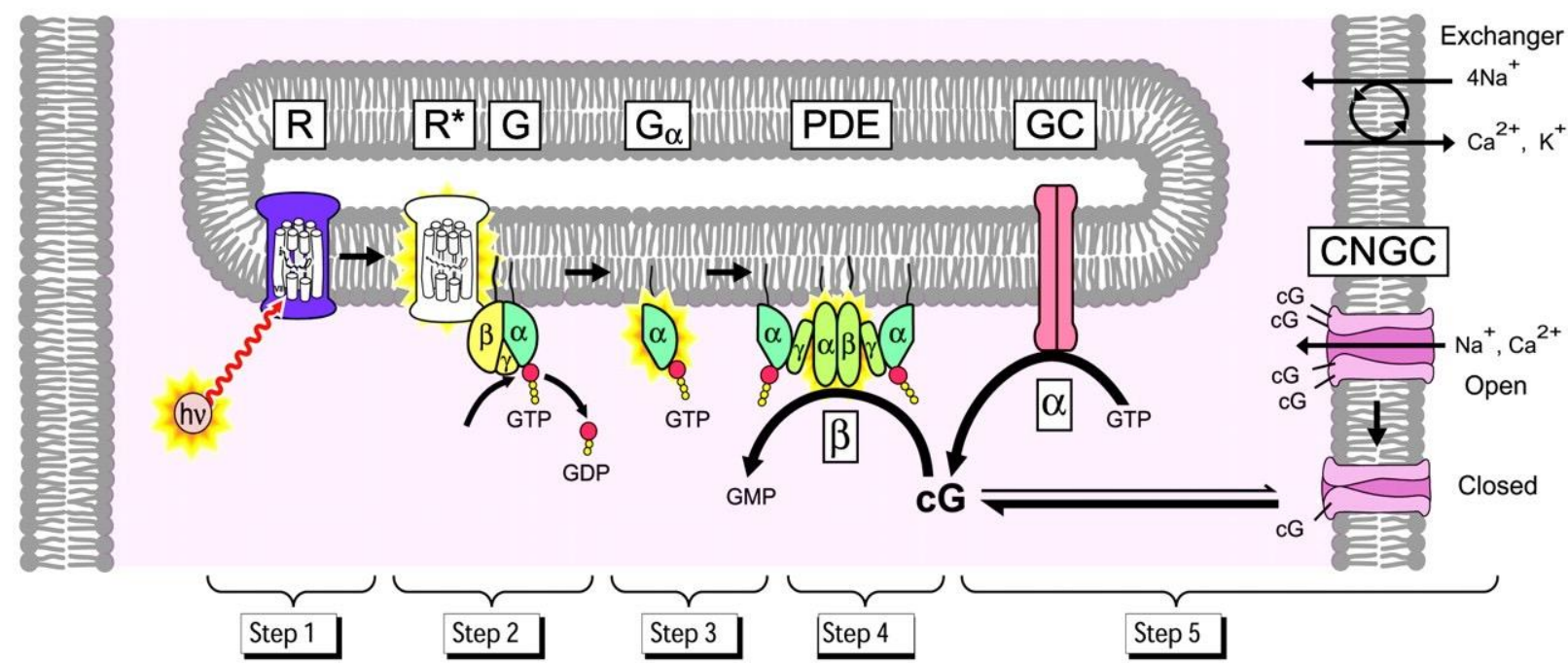

Figure. 3 Rod phototransduction cascade.

Light-activated rhodopsin (step1) interacts with heterotrimeric transducin (step2) resulting in the exchange of GDP for GTP on Ta. Nucleotide exchange results in dissociation of Ta and the obligate T $\beta \gamma$ heterodimer. GTP bound cytosolic T $\alpha$ activates PDE6 (step3), resulting in hydrolysis of cGMP produced by guanylyl cyclase. Intracellular concentrations of cGMP are lowered leading to closure of CNGA channels and hyperpolarization of the plasma membrane. Adapted from (2) 
phosphorylation of rhodopsin. Arrestin (Arr) binds phosphorylated rhodopsin, inhibiting its interaction with transducin $(1,2,13)$. As stated earlier, three of the above proteins, rod $\mathrm{G} \gamma$, PDE6 $\alpha \beta$, and GRK1, are prenylated and undergo “ $C A A X$ ” processing $(37,38,50)$.

\section{G-protein transducin complex}

Transducin is a membrane-bound heterotrimer containing $\alpha, \beta$ and $\gamma$ subunits, and in its inactive state, it is bound to a guanosine diphosphate (GDP) molecule (67-70). Light dependent activation by rhodopsin promotes the binding of guanosine triphosphate (GTP) and results in detachment of the $\alpha$ subunit from the membrane, which results in the activation of PDE6. The remaining subunits $(\mathrm{G} \beta \gamma)$ then diffuse to the IS of the rod photoreceptor $(1,13)$. Amplification of signaling requires proper assembly and localization of transducin in relationship to its signaling partners. This is likely achieved through a series of PTMs which includes myristoylation of the catalytic $\mathrm{G} \alpha$-subunit and farnesylation of the $\mathrm{G} \gamma$ subunit of the obligate $\mathrm{G}-\alpha \beta \gamma$ complex in both rods and cones (52). Peripheral membrane proteins generally require at least two hydrophobic lipid group signals for membrane association. Several lines of evidence suggest lipid PTMs of transducin are essential for its membrane association. First, the absence of the myristoylated group on rod $\mathrm{G} \alpha \mathrm{T} 1$ resulted in a gross mislocalization as well as a marked reduction in phototransduction (71). Furthermore, genetic elimination of rod specific G $\gamma \mathrm{T} 1$ resulted in increased solubility of GaT1 and decreased amplification of the signal transduction cascade (72). The single prenyl group alone is insufficient for assembly and localization of heterotrimeric Gproteins in vitro. In addition to a prenyl group, methylation is also required for membrane association. ICMT-deficient fibroblast in retina demonstrated farnesylated rod G $\gamma \mathrm{T} 1$ requires methyl esterification for endomembrane association, while the more hydrophobic geranylgeranylated $\mathrm{G} \gamma \mathrm{T} 1$ does not (73). In-vivo studies utilizing retina lacking ICMT in mice 
(59), showed that methylation of $\mathrm{G} \gamma \mathrm{T} 1$ is essential for its proper membrane association and signal transduction. Collectively, this data suggests that rod transducin requires myristoylation of

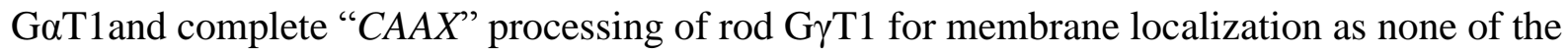
modifications alone are sufficient.

Although the $C A A X$ motif is present on the $\mathrm{G} \alpha$ and $\mathrm{G} \gamma$ subunits of rod transducin, only the $\mathrm{G} \gamma$ subunit has been found to be farnesylated $(36,51)$. This modification is necessary for efficient translocation in rods (74). During dark adaptation, dislocated transducin subunits must return to the OS for rod sensitivity restoration. It was established that this process may be mediated by the farnesyl residue (74). We recently demonstrated that in the absence of farnesyltransferase $(F n t b)$ in mice retina, the translocation kinetics of $\mathrm{T} \beta \gamma$ in rod photoreceptors cells are impaired. This impairment leads to progressive loss of photoreceptor function and photoreceptor degeneration (chapter 3 of this thesis). The experiments with mice models lacking the gene encoding the rod transducin $\gamma$ subunit, resulted in severe down-regulation of $\alpha$ and $\beta$ subunits of transducin, despite normal mRNA levels, and rapid photoreceptor degeneration (75). Rod T $\gamma$ encoding gene, GNGT1, has previously been suggested to play a role in human retinal degeneration disorders, but thus far no mutations have been found in this gene in human retinal

disorders. Mutations in the gene encoding the transducin $\alpha$ subunit, were identified in congenital stationary night blindness $(76,77)$. These findings, along with the lack of prenylation of rod $\mathrm{T} \gamma$ that leads to photoreceptor degeneration in one of our mouse models, suggest that mutations in GNGT1 may be involved in a similar disease.

\section{Phosphodiesterase6 (PDE6)}

Rod PDE6, the effector enzyme downstream of transducin, is a heterotetramer composed of a farnesylated $\alpha$ subunit, a geranylgeranylated $\beta$ subunit as well as two inhibitory $\gamma$ subunits 
$(38,78)$. In contrast, Cone PDE6 is a heterotetramer composed of two catalytic subunits ( $\left.\alpha^{\prime}\right)$ and two inhibitory subunits $(\gamma)$. Cone PDE6, is thought to be geranylgeranylated based on the $-C A A X$ sequence analysis $(12,41,78-80)$. Rod PDE6 is the only known protein to be differentially prenylated, however its contribution to PDE6 function has not been conclusively determined (39). Evidence from a patient with compound heterozygous mutations in PDE $\beta$ suggests differential prenylation is crucial to rod function (81). A mutation in one allele introduces a stop codon producing a non-functional protein which by itself would not be problematic; however, the other allele has a mutation in the " $C A A X$ " motif that is predicted to substitute a valine for leucine at the X position. Valine is predicted to specify farnesylation instead of geranylgeranylation $(14,81)$. These findings implicate differential prenylation in the maintenance of high fidelity phototransduction in rod photoreceptors.

The final two steps of " $C A A X$ " protein processing also appear to be critical to PDE6 transport and maintenance in the OS. Purifications of PDE6 identified a putative fourth subunit, later referred to as prenyl binding protein/delta $\operatorname{PrBP} \delta$ (79). It has since been shown to be able to solubilize PDE6 and thought to be important for transporting PDE6 to the OS $(28,30)$. To identify the site of interaction between PDE6 and $\operatorname{PrBP} \delta$, peptides corresponding to the last six amino acids of PDE6 catalytic subunits were tested for binding affinity with PrBP $\delta$. Prenylated and methylated peptides were able to interact with $\operatorname{PrBP} \delta$, however lack of either prenylation or methyl esterification was sufficient to abolish this interaction in vitro $(28,29,57)$.

The importance of " $C A A X$ ” processing for PDE6 function was recently shown in vivo. In a mouse model lacking RCE1-mediated endoproteolysis in the retina, a specific accumulation of PDE6 was observed below the CC (46). Interestingly, absence of ICMT-mediated methyl esterification did not affect the transport of PDE6 to OS, but did affect PDE6 protein stability in 
the OS (59). The same study also demonstrates that lack of methylation results in defective membrane association of cone PDE6. Overall, these results implicate the importance of prenylation and postprenylation processing in the transport, maintenance and function of PDE6. To understand the in-vivo importance of prenylation in rod photoreceptors, we generated the conditional knockout (CKO) mouse model lacking prenylation in retinal neurons (chapter 3). These studies highlight that although the assembly of rod PDE6 is not affected in the absence of protein farnesylation or geranylgeranylation, the protein levels and membrane association of rod PDE6 is significantly affected leading to progressive loss of photoreceptor function and photoreceptor degeneration.

In contrast to rod PDE6, cone photoreceptor cells are predicted to utilize a geranylgeranylated $\alpha^{\prime}$ homodimer to form their holoenzyme $(12,41,82)$. In the studies, with cone photoreceptor specific CKO of prenyl transferases, in the absence of geranylgeranylation, the cone function was abolished at one month of age. This was accompanied with drastic reduction in cone PDE6 levels. Lack of protein geranylgeranylation lead to impaired membrane anchorage of cone PDE6. Interestingly, cone PDE6 failed to assemble in the absence of Pggtlb. These studies highlight Pggt1b mediated geranylgeranylation is crucial for cone PDE6 assembly and cone photoreceptor function (Pendse et al. 2016, unpublished findings).

\section{Aryl-hydrocarbon-interacting protein-like 1 (AIPL1) and Rod PDE6}

Aryl-hydrocarbon-interacting protein-like 1 (AIPL1) is thought to function as a chaperone for farnesylated proteins. AIPL1 is expressed in both rods and cones in the early stages of development, whereas in later stages the expression is restricted to rods. Earlier studies with complete knockouts of AIPL1 showed dramatic photoreceptor degeneration. AIPL1 has been found to be mutated in persons with LCA or cone-rod dystrophy. AIPL1 has been shown to 
interact with DNAJA2 which is DNAJ family protein, rod specific transducin $\gamma$ subunit in yeast and the $\alpha$ and $\beta$ subunits of rod PDE6 in mice, in the presence of FTases $(8,9,83)$. The underlying molecular mechanism behind degeneration of both rods and cones in mice model lacking AIPL1, is due to disassembly of PDE6. Detailed biochemical analysis showed that in absence of AIPL1, rod PDE6 subunits (catalytic $\alpha, \beta$ and inhibitory $\gamma$ ) are synthesized normally but are not stable and do not assemble properly (84-86). Misassembled PDE6 subunits are likely to be rapidly degraded by proteasome. Interestingly, it was observed that immunoprecipitation with AIPL1 specific monoclonal antibodies show PDE6 $\alpha$ as the primary interacting partner. Although AIPL1 is thought to be involved in folding of PDE6 subunits, the importance of farnesylation of PDE6 $\alpha$ in interaction with AIPL1 is poorly understood.

\section{AIPL1 and Cone PDE6}

The study of PDE6 has been slow because the creation of a heterologous system, such as tissue culture or retinal cell lines, has not been accomplished $(10,64)$. In cones, PDE6 is thought to be geranylgeranylated. Although AIPL1 is not expressed in adult mouse cones, AIPL1 might play crucial role in cones. In AIPL1 complete knockout mice, cone mediated-photopic ERG response was completely absent at any age tested. Using transgenic mice model with human AIPL1 expression in rods, it was shown that with the absence of rod degeneration, cone cells were lost but at a much slower rate signifying a direct and an important role for AIPL1 in cones (85-87). Slower rate of cone degeneration in these animal models suggests that rapid rod cell death was partly responsible for fast rate of cone cell death observed in mice with complete knockout of AIPL1. This finding suggests a role for AIPL1 in stability of cone PDE6 subunits $(82,85)$. It also showed that AIPL1 interacts with cone PDE6 catalytic subunits thought to be 
geranylgeranylated. Our recent observations with prenyl transferases knockouts in mice retina, suggest that lipid anchors, farnesyl or geranylgeranyl, acts as a molecular grip facilitating the interaction AIPL1 and PDE6.

\section{G-protein coupled receptor kinase or rhodopsin kinase (GRK1)}

Rhodopsin signaling is inactivated by phosphorylation of rhodopsin by GRK1. Phosphorylation allows for the binding of arrestin, which blocks rhodopsin's interaction with transducin (Fig. 4). It has been shown that GRK1 is a monomeric kinase which is farnesylated and methyl esterified in vitro $(37,88,89)$. Mutations in GRK1 lead to Oguchi disease in humans, a form of congenital stationary night blindness (90). In the knockout of $\operatorname{PrBP} \delta$, reduced levels of GRK1 were observed in mouse retinas. (36) $\operatorname{PrBP} \delta$, a chaperone, binds to multiple prenylated proteins (28-30), but the specific reduction in levels of GRK1 strongly suggests a requirement for prenylation and postprenylation processing in its maintenance and transport. Fascinatingly, the membrane association of GRK1 occurs after its farnesylation (29). Our recent observation in the mice retina lacking prenyl transferases, the GRK1 protein levels and membrane association were severely affected suggesting the importance of prenylation in stability and membrane anchorage of GRK1. Similarly, ICMT CKO animal models illustrate the importance of methylation in the GRK1 stability in cones (59).

\section{RAB28 (Ras-related in brain) and CLN3 (Ceroid Lipofuscinosis Neuronal 3)}

RAB28 is a member of the Rab subfamily of small GTPases. It was only recently implicated in photoreceptor function through a genomic analysis in multiple families identifying mutations in the $R A B 28$ gene that result in cone-rod dystrophy $(91,92)$. Rab GTPases are involved in a wide range of cellular processes mostly involving dynamics of vesicle trafficking, vesicle fusion, and 
membrane fission events. However, whether Rab28 plays a role in these processes is yet to be determined. Although the exact role of RAB28 in cones and rods is poorly understood, isoforms 1 and 2 of RAB28 are predicted to be farnesylated at their $C A A X$ sequence (91-93).

This is in contrast to majority of other RAB GTPases that are geranylgeranylated by enzyme GGTase-II (31). A few other members of Rab family, Rab 3a, Rab8 and Rab11, are also found to be involved in rhodopsin trafficking and ciliogenesis (1). In addition, Rab17 and Rab23 were found to be essential for ciliogenesis $(35,92,94-96)$. Of note, RAB28 is localized to the basal body and ciliary rootlet, suggesting its putative role in ciliogenesis or ciliary transport $(91,97)$. The underlying mechanism behind photoreceptor degeneration due to mutations in RAB28 remains to be elucidated. The retina specific phenotype is surprising in view of the wide expression of this gene throughout the human body, however protein prenylation in stability and function of RAB28 is something that remains to be investigated.

CLN3 encodes a lysosomal protein, which if mutated results in Batten disease. The first clinical symptoms of this disease are often vision loss and progressive neurodegeneration (98101). A recent study shows a patient mutation in CLN3 results into a non-syndromic LCA $(99,100,102)$ The CLN3 protein is thought to be farnesylated (40) and it has been shown that mutations in the CLN3-binding motif of FTase results in impaired protein.

\section{Defects in prenylation lead to retinal degenerative diseases}

The underlying mechanisms of retinal degeneration due to defects in the prenylated proteins as described above are not fully understood, but can be inferred from their known roles in the phototransduction cascade (transducin and PDE6), transport processes at the connecting cilium (RAB28), or lysosomes (CLN3). The importance of prenylation in photoreceptor neurons 
is highlighted by the fact that defects in this process cause choroideremia, a disease primarily affecting rods (103).

A patient mutation in the amino acid at $\mathrm{C}$-terminal end of the PDE6 $\beta$ subunit provides clinical evidence of the importance of differential PDE6 lipid modifications. Based on studies of prenyl transferase " $C A A X$ " box specificity, the mutation is predicted to alter the prenyl group from a geranylgeranyl to a farnesyl group. Animal models phenocopying a similar mutation in PDE6 $\beta$ show progressive rod cell degeneration and dysfunction (Singh and Ramamurthy, unpublished findings). The prenyl moieties of PDE6 subunits were recently shown to bind AIPL1, the chaperone protein involved in LCA $(8,9,83)$. PDE6 subunits form a complex containing prenyl binding protein delta $(\mathrm{PrBP} / \delta)$, which is highly expressed in photoreceptors. Mutations in both PDE6A and PDE6B (phosphodiesterase 6A, cGMP-specific, rod, $\alpha$ and $\beta$, respectively), encoding the $\alpha$ and $\beta$ subunits, are responsible for a small subset of autosomalrecessive RP cases. Further evidence for the importance of prenylation in retinal function comes from a study showing a link between changes in rod PDE $\beta$ subunit and rod-cone dysplasia (104). In this canine model for RP, a non-sense mutation that removed the last 49 amino acid residues including the " $C A A X$ " box resulted in non-functional PDE and severe degeneration of rods (105). Additionally, the mutations affecting chaperone AIPL1 and PrBP/ $\delta$ exert a more detrimental effect, since both serve as chaperones for the above-mentioned prenylated retinal proteins $(8,9,28-30,85,86)$. Altogether, these studies demonstrate the importance of protein prenylation in proper functioning and survival of retinal neurons. 


\section{REFERENCES}

1 Pearring, J.N., Salinas, R.Y., Baker, S.A. and Arshavsky, V.Y. (2013) Protein sorting, targeting and trafficking in photoreceptor cells. Progress in retinal and eye research, 36, 24-51. 2 Lamb, T.D. and Pugh, E.N. (2006) Phototransduction, dark adaptation, and rhodopsin regeneration the proctor lecture. Investigative ophthalmology \& visual science, 47, 5138-5152. 3 Young, R.W. (1967) The renewal of photoreceptor cell outer segments. The Journal of cell biology, 33, 61-72.

4 Oesterhelt, D. and Stoeckenius, W. (1973) Functions of a new photoreceptor membrane. Proceedings of the National Academy of Sciences, 70, 2853-2857.

5 Linton, J.D., Holzhausen, L.C., Babai, N., Song, H., Miyagishima, K.J., Stearns, G.W., Lindsay, K., Wei, J., Chertov, A.O. and Peters, T.A. (2010) Flow of energy in the outer retina in darkness and in light. Proceedings of the National Academy of Sciences, 107, 8599-8604.

6 Besharse, J.C., Hollyfield, J.G. and Rayborn, M.E. (1977) Turnover of rod photoreceptor outer segments. II. Membrane addition and loss in relationship to light. The Journal of cell biology, 75, 507-527.

7 Yamamoto, H., Simon, A., Eriksson, U., Harris, E., Berson, E.L. and Dryja, T.P. (1999) Mutations in the gene encoding 11-cis retinol dehydrogenase cause delayed dark adaptation and fundus albipunctatus. Nature genetics, 22, 188-191.

8 Schwartz, M.L., Hurley, J.B. and Ramamurthy, V. (2006), In Retinal Degenerative Diseases. Springer, in press., pp. 89-94.

9 Ramamurthy, V., Roberts, M., van den Akker, F., Niemi, G., Reh, T. and Hurley, J.B. (2003) AIPL1, a protein implicated in Leber's congenital amaurosis, interacts with and aids in processing of farnesylated proteins. Proceedings of the National Academy of Sciences, $\mathbf{1 0 0}$, 12630-12635.

10 Muradov, H., Boyd, K.K., Haeri, M., Kerov, V., Knox, B.E. and Artemyev, N.O. (2009) Characterization of human cone phosphodiesterase-6 ectopically expressed in Xenopus laevis rods. Journal of Biological Chemistry, 284, 32662-32669.

11 Deretic, D. (2006) A role for rhodopsin in a signal transduction cascade that regulates membrane trafficking and photoreceptor polarity. Vision research, 46, 4427-4433.

12 Baehr, W. (2014) Membrane Protein Transport in Photoreceptors: The Function of PDE $\delta$ The Proctor Lecture. Investigative ophthalmology \& visual science, 55, 8653-8666.

13 Calvert, P.D., Strissel, K.J., Schiesser, W.E., Pugh, E.N. and Arshavsky, V.Y. (2006) Light-driven translocation of signaling proteins in vertebrate photoreceptors. Trends in cell biology, 16, 560-568.

14 Hartman, H.L., Hicks, K.A. and Fierke, C.A. (2005) Peptide specificity of protein prenyltransferases is determined mainly by reactivity rather than binding affinity. Biochemistry, 44, 15314-15324.

15 Zhang, F.L. and Casey, P.J. (1996) Protein prenylation: molecular mechanisms and functional consequences. Annual review of biochemistry, 65, 241-269.

16 Bergo, M.O., Lieu, H.D., Gavino, B.J., Ambroziak, P., Otto, J.C., Casey, P.J., Walker, Q.M. and Young, S.G. (2004) On the Physiological Importance of Endoproteolysis of CAAX Proteins heart-specific rce1 knockout mice develop a lethal cardiomyopathy. Journal of Biological Chemistry, 279, 4729-4736. 
17 Kim, E., Ambroziak, P., Otto, J.C., Taylor, B., Ashby, M., Shannon, K., Casey, P.J. and Young, S.G. (1999) Disruption of the mouse Rcel gene results in defective Ras processing and mislocalization of Ras within cells. J Biol Chem, 274, 8383-8390.

18 Wahlstrom, A.M., Cutts, B.A., Karlsson, C., Andersson, K.M., Liu, M., Sjogren, A.K., Swolin, B., Young, S.G. and Bergo, M.O. (2007) Rce1 deficiency accelerates the development of K-RAS-induced myeloproliferative disease. Blood, 109, 763-768.

19 Cox, A.D. and Der, C.J. (1992) Protein prenylation: more than just glue? Current opinion in cell biology, 4, 1008-1016.

20 Hrycyna, C., Bergo, M.O. and Tamanoi, F. (2011) Protein Prenylation. Academic Press.

21 Roosing, S., Collin, R.W., den Hollander, A.I., Cremers, F.P. and Siemiatkowska, A.M.

(2014) Prenylation defects in inherited retinal diseases. Journal of medical genetics, 51, 143-151.

22 Seabra, M.C., Ho, Y.K. and Anant, J.S. (1995) Deficient geranylgeranylation of

Ram/Rab27 in choroideremia. Journal of Biological Chemistry, 270, 24420-24427.

23 Winter-Vann, A.M. and Casey, P.J. (2005) Post-prenylation-processing enzymes as new

targets in oncogenesis. Nat Rev Cancer, 5, 405-412.

24 Magee, A.I. and Seabra, M.C. (2003) Are prenyl groups on proteins sticky fingers or greasy handles? Biochem J, 376, e3-4.

25 Kloog, Y. and Cox, A.D. (2004) Prenyl-binding domains: potential targets for Ras inhibitors and anti-cancer drugs. Semin Cancer Biol, 14, 253-261.

26 Norton, A.W., Hosier, S., Terew, J.M., Li, N., Dhingra, A., Vardi, N., Baehr, W. and Cote, R.H. (2005) Evaluation of the 17-kDa prenyl-binding protein as a regulatory protein for phototransduction in retinal photoreceptors. J Biol Chem, 280, 1248-1256.

27 Norton, A.W., Hosier, S., Terew, J.M., Li, N., Dhingra, A., Vardi, N., Baehr, W. and Cote, R.H. (2005) Evaluation of the 17-kDa prenyl-binding protein as a regulatory protein for phototransduction in retinal photoreceptors. Journal of Biological Chemistry, 280, 1248-1256. 28 Zhang, H., Hosier, S., Terew, J.M., Zhang, K., Cote, R.H. and Baehr, W. (2005) Assay and functional properties of $\operatorname{PrBP}(\mathrm{PDEdelta})$, a prenyl-binding protein interacting with multiple partners. Methods Enzymol, 403, 42-56.

29 Zhang, H., Li, S., Doan, T., Rieke, F., Detwiler, P., Frederick, J. and Baehr, W. (2007) Deletion of $\mathrm{PrBP} / \delta$ impedes transport of GRK1 and PDE6 catalytic subunits to photoreceptor outer segments. Proceedings of the National Academy of Sciences, 104, 8857-8862.

30 Zhang, H., Li, S., Doan, T., Rieke, F., Detwiler, P.B., Frederick, J.M. and Baehr, W. (2007) Deletion of PrBP/delta impedes transport of GRK1 and PDE6 catalytic subunits to photoreceptor outer segments. Proc Natl Acad Sci U S A, 104, 8857-8862.

31 Casey, P.J. and Seabra, M.C. (1996) Protein prenyltransferases. Journal of Biological Chemistry, 271, 5289-5292.

32 Magee, A.I. and Seabra, M.C. (2003) Are prenyl groups on proteins sticky fingers or greasy handles? Biochemical Journal, 376, e3-e4.

33 Mijimolle, N., Velasco, J., Dubus, P., Guerra, C., Weinbaum, C.A., Casey, P.J., Campuzano, V. and Barbacid, M. (2005) Protein farnesyltransferase in embryogenesis, adult homeostasis, and tumor development. Cancer cell, 7, 313-324.

34 Sjogren, A.-K.M., Andersson, K.M., Liu, M., Cutts, B.A., Karlsson, C., Wahlstrom, A.M., Dalin, M., Weinbaum, C., Casey, P.J. and Tarkowski, A. (2007) GGTase-I deficiency reduces tumor formation and improves survival in mice with K-RAS-induced lung cancer. The Journal of clinical investigation, 117, 1294-1304. 
35 Larijani, B., Hume, A.N., Tarafder, A.K. and Seabra, M.C. (2003) Multiple factors contribute to inefficient prenylation of Rab27a in Rab prenylation diseases. Journal of Biological Chemistry, 278, 46798-46804.

36 Fukada, Y., Takao, T., Ohguro, H., Yoshizawa, T., Akino, T. and Shimonishi, Y. (1990) Farnesylated gamma-subunit of photoreceptor $\mathrm{G}$ protein indispensable for GTP-binding. Nature, 346, 658-660.

37 Inglese, J., Koch, W.J., Caron, M.G. and Lefkowitz, R.J. (1992) Isoprenylation in regulation of signal transduction by G-protein-coupled receptor kinases. Nature, 359, 147-150.

38 Anant, J.S., Ong, O.C., Xie, H.Y., Clarke, S., O'Brien, P.J. and Fung, B.K. (1992) In vivo differential prenylation of retinal cyclic GMP phosphodiesterase catalytic subunits. J Biol Chem, 267, 687-690.

39 Ong, O.C., Ota, I.M., Clarke, S. and Fung, B.K. (1989) The membrane binding domain of rod cGMP phosphodiesterase is posttranslationally modified by methyl esterification at a Cterminal cysteine. Proc Natl Acad Sci U S A, 86, 9238-9242.

40 Pullarkat, R.K. and Morris, G.N. (1997) Farnesylation of Batten disease CLN3 protein. Neuropediatrics, 28, 42-44.

41 Gillespie, P.G. and Beavo, J.A. (1988) Characterization of a bovine cone photoreceptor phosphodiesterase purified by cyclic GMP-sepharose chromatography. Journal of Biological Chemistry, 263, 8133-8141.

42 Zhang, F.L. and Casey, P.J. (1996) Protein prenylation: molecular mechanisms and functional consequences. Annu Rev Biochem, 65, 241-269.

43 Bergo, M.O., Lieu, H.D., Gavino, B.J., Ambroziak, P., Otto, J.C., Casey, P.J., Walker, Q.M. and Young, S.G. (2004) On the physiological importance of endoproteolysis of CAAX proteins: heart-specific RCE1 knockout mice develop a lethal cardiomyopathy. J Biol Chem, 279, 4729-4736.

44 Michaelson, D., Ali, W., Chiu, V.K., Bergo, M., Silletti, J., Wright, L., Young, S.G. and Philips, M. (2005) Postprenylation CAAX processing is required for proper localization of Ras but not Rho GTPases. Mol Biol Cell, 16, 1606-1616.

45 Christiansen, J.R., Kolandaivelu, S., Bergo, M.O. and Ramamurthy, V. (2011) RASconverting enzyme 1-mediated endoproteolysis is required for trafficking of rod phosphodiesterase 6 to photoreceptor outer segments. Proc Natl Acad Sci U S A, in press.

46 Hrycyna, C.A., Sapperstein, S.K., Clarke, S. and Michaelis, S. (1991) The Saccharomyces cerevisiae STE14 gene encodes a methyltransferase that mediates C-terminal methylation of a-factor and RAS proteins. EMBO J, 10, 1699-1709.

47 Griggs, A.M., Hahne, K. and Hrycyna, C.A. (2010) Functional oligomerization of the Saccharomyces cerevisiae isoprenylcysteine carboxyl methyltransferase, Ste14p. J Biol Chem, 285, 13380-13387.

48 Anderson, J.L. and Hrycyna, C.A. (2006) 9 Structure and function of isoprenylcysteine carboxylmethyltransferase (Icmt): A key enzyme in CaaX processing. The Enzymes, 24, 245272.

49 Swanson, R.J. and Applebury, M.L. (1983) Methylation of proteins in photoreceptor rod outer segments. J Biol Chem, 258, 10599-10605.

50 Ohguro, H., Fukada, Y. and Akino, T. (1991) Structure and function of gamma-subunit of photoreceptor G-protein (transducin). Comp Biochem Physiol B, 100, 433-438. 
51 Fukada, Y., Takao, T., Ohguro, H., Yoshizawa, T., Akino, T. and Shimonishi, Y. (1990) Farnesylated gamma-subunit of photoreceptor G protein indispensable for GTP-binding. Nature, 346, 658-660.

52 Bergo, M.O., Leung, G.K., Ambroziak, P., Otto, J.C., Casey, P.J., Gomes, A.Q., Seabra, M.C. and Young, S.G. (2001) Isoprenylcysteine carboxyl methyltransferase deficiency in mice. $J$ Biol Chem, 276, 5841-5845.

53 Svensson, A.W., Casey, P.J., Young, S.G. and Bergo, M.O. (2006) Genetic and pharmacologic analyses of the role of Icmt in ras membrane association and function. Methods in enzymology, 407, 144-159.

54 Bergo, M.O., Gavino, B.J., Hong, C., Beigneux, A.P., McMahon, M., Casey, P.J. and Young, S.G. (2004) Inactivation of Icmt inhibits transformation by oncogenic K-Ras and B-Raf. J Clin Invest, 113, 539-550.

55 Parish, C.A. and Rando, R.R. (1996) Isoprenylation/methylation of proteins enhances membrane association by a hydrophobic mechanism. Biochemistry, 35, 8473-8477.

56 Cook, T.A., Ghomashchi, F., Gelb, M.H., Florio, S.K. and Beavo, J.A. (2000) Binding of the delta subunit to rod phosphodiesterase catalytic subunits requires methylated, prenylated Ctermini of the catalytic subunits. Biochemistry, 39, 13516-13523.

57 Ohguro, H., Fukada, Y., Takao, T., Shimonishi, Y., Yoshizawa, T. and Akino, T. (1991) Carboxyl methylation and farnesylation of transducin gamma-subunit synergistically enhance its coupling with metarhodopsin II. The EMBO journal, 10, 3669.

58 Christiansen, J.R., Pendse, N.D., Kolandaivelu, S., Bergo, M.O., Young, S.G. and Ramamurthy, V. (2016) Deficiency of Isoprenylcysteine Carboxyl Methyltransferase (ICMT) Leads to Progressive Loss of Photoreceptor Function. The Journal of Neuroscience, 36, 51075114 .

59 Linton, J.D., Holzhausen, L.C., Babai, N., Song, H., Miyagishima, K.J., Stearns, G.W., Lindsay, K., Wei, J., Chertov, A.O., Peters, T.A. et al. (2010) Flow of energy in the outer retina in darkness and in light. Proc Natl Acad Sci U S A, 107, 8599-8604.

60 Young, R.W. (1967) The renewal of photoreceptor cell outer segments. J Cell Biol, 33, $61-72$.

61 Liu, Q., Tan, G., Levenkova, N., Li, T., Pugh, E.N., Jr., Rux, J.J., Speicher, D.W. and Pierce, E.A. (2007) The proteome of the mouse photoreceptor sensory cilium complex. Mol Cell Proteomics, 6, 1299-1317.

62 Artemyev, N.O., Natochin, M., Busman, M., Schey, K.L. and Hamm, H.E. (1996) Mechanism of photoreceptor cGMP phosphodiesterase inhibition by its gamma-subunits. Proceedings of the National Academy of Sciences, 93, 5407-5412.

63 Muradov, K.G., Granovsky, A.E., Schey, K.L. and Artemyev, N.O. (2002) Direct interaction of the inhibitory $\gamma$-subunit of rod cGMP phosphodiesterase (PDE6) with the PDE6 GAFa domains. Biochemistry, 41, 3884-3890.

64 Tcheudji, J.F.K., Lebeau, L., Virmaux, N., Maftei, C.G., Cote, R.H., Lugnier, C. and Schultz, P. (2001) Molecular organization of bovine rod cGMP-phosphodiesterase 6. Journal of molecular biology, 310, 781-791.

65 Burns, M.E. and Arshavsky, V.Y. (2005) Beyond counting photons: trials and trends in vertebrate visual transduction. Neuron, 48, 387-401.

66 Angleson, J.K. and Wensel, T.G. (1993) A GTPase-accelerating factor for transducin, distinct from its effector cGMP phosphodiesterase, in rod outer segment membranes. Neuron, 11, 939-949. 
67 Lobanova, E.S., Finkelstein, S., Song, H., Tsang, S.H., Chen, C.-K., Sokolov, M., Skiba, N.P. and Arshavsky, V.Y. (2007) Transducin translocation in rods is triggered by saturation of the GTPase-activating complex. The Journal of neuroscience, 27, 1151-1160.

68 Matsuda, T., Takao, T., Shimonishi, Y., Murata, M., Asano, T., Yoshizawa, T. and Fukada, Y. (1994) Characterization of interactions between transducin alpha/beta gammasubunits and lipid membranes. Journal of Biological Chemistry, 269, 30358-30363.

69 Skiba, N.P., Hopp, J.A. and Arshavsky, V.Y. (2000) The effector enzyme regulates the duration of $\mathrm{G}$ protein signaling in vertebrate photoreceptors by increasing the affinity between transducin and RGS protein. Journal of Biological Chemistry, 275, 32716-32720.

70 Kerov, V., Rubin, W.W., Natochin, M., Melling, N.A., Burns, M.E. and Artemyev, N.O. (2007) N-terminal fatty acylation of transducin profoundly influences its localization and the kinetics of photoresponse in rods. J Neurosci, 27, 10270-10277.

71 Kolesnikov, A.V., Rikimaru, L., Hennig, A.K., Lukasiewicz, P.D., Fliesler, S.J., Govardovskii, V.I., Kefalov, V.J. and Kisselev, O.G. (2011) G-protein betagamma-complex is crucial for efficient signal amplification in vision. $J$ Neurosci, 31, 8067-8077.

72 Michaelson, D., Ahearn, I., Bergo, M., Young, S. and Philips, M. (2002) Membrane trafficking of heterotrimeric $\mathrm{G}$ proteins via the endoplasmic reticulum and Golgi. Mol Biol Cell, 13, 3294-3302.

73 Kassai, H., Aiba, A., Nakao, K., Nakamura, K., Katsuki, M., Xiong, W.-H., Yau, K.-W., Imai, H., Shichida, Y. and Satomi, Y. (2005) Farnesylation of retinal transducin underlies its translocation during light adaptation. Neuron, 47, 529-539.

74 Finkelstein, S., Lobanova, E. and Arshavsky, V. (2009) Knockout of the Rod Transducin Gamma Subunit Induces Cell Death. Investigative Ophthalmology \& Visual Science, 50, 44724472.

75 Miyake, Y., Yagasaki, K., Horiguchi, M., Kawase, Y. and Kanda, T. (1986) Congenital stationary night blindness with negative electroretinogram: a new classification. Archives of ophthalmology, 104, 1013-1020.

76 Sandberg, M.A., Pawlyk, B.S., Dan, J., Arnaud, B., Dryja, T.P. and Berson, E.L. (1998) Rod and cone function in the Nougaret form of stationary night blindness. Archives of Ophthalmology, 116, 867-872.

77 Baehr, W., Devlin, M.J. and Applebury, M.L. (1979) Isolation and characterization of cGMP phosphodiesterase from bovine rod outer segments. J Biol Chem, 254, 11669-11677. 78 Gillespie, P.G., Prusti, R.K., Apel, E.D. and Beavo, J.A. (1989) A soluble form of bovine rod photoreceptor phosphodiesterase has a novel 15-kDa subunit. J Biol Chem, 264, 1218712193.

79 Cote, R.H. (2006) Photoreceptor phosphodiesterase (PDE6): a G-protein-activated PDE regulating visual excitation in rod and cone photoreceptor cells. in press.

80 Veske, A., Orth, U., Ruther, K., Zrenner, E., Rosenberg, T., Baehr, W., Gal, A. (1995) Mutations in the gene fro the b-subunit of rod photoreceptor cgmp-specific phosphodiesterase (PDEB) in patients with retinal dystrophies and dysfunctions. Degenerative Diseases of the Retina, in press., 313-322.

81 Kolandaivelu, S., Chang, B. and Ramamurthy, V. (2011) Rod Phosphodiesterase-6 (PDE6) catalytic subunts restores cone function in a mouse model lacking cone PDE6 catalytic subunit. J Biol Chem, in press.

82 Ramamurthy, V., Niemi, G.A., Reh, T.A. and Hurley, J.B. (2004) Leber congenital amaurosis linked to AIPL1: a mouse model reveals destabilization of cGMP phosphodiesterase. 
Proceedings of the National Academy of Sciences of the United States of America, 101, 1389713902.

83 Kolandaivelu, S., Huang, J., Hurley, J.B. and Ramamurthy, V. (2009) AIPL1, a protein associated with childhood blindness, interacts with $\alpha$-subunit of rod phosphodiesterase (PDE6) and is essential for its proper assembly. Journal of Biological Chemistry, 284, 30853-30861.

84 Kolandaivelu, S. and Ramamurthy, V. (2014), In Retinal Degenerative Diseases. Springer, in press., pp. 43-48.

85 Kolandaivelu, S., Singh, R.K. and Ramamurthy, V. (2013) AIPL1, A protein linked to blindness, is essential for the stability of enzymes mediating cGMP metabolism in cone photoreceptor cells. Human molecular genetics, in press., ddt496.

86 Kirschman, L.T., Kolandaivelu, S., Frederick, J.M., Dang, L., Goldberg, A.F., Baehr, W. and Ramamurthy, V. (2009) The Leber congenital amaurosis protein, AIPL1, is needed for the viability and functioning of cone photoreceptor cells. Human molecular genetics, in press., ddp571.

87 Horner, T.J., Osawa, S., Schaller, M.D. and Weiss, E.R. (2005) Phosphorylation of GRK1 and GRK7 by cAMP-dependent protein kinase attenuates their enzymatic activities. Journal of Biological Chemistry, 280, 28241-28250.

88 Weiss, E.R., Ducceschi, M.H., Horner, T.J., Li, A., Craft, C.M. and Osawa, S. (2001) Species-specific differences in expression of G-protein-coupled receptor kinase (GRK) 7 and GRK1 in mammalian cone photoreceptor cells: implications for cone cell phototransduction. The Journal of Neuroscience, 21, 9175-9184.

89 Chen, C.K., Burns, M.E., Spencer, M., Niemi, G.A., Chen, J., Hurley, J.B., Baylor, D.A. and Simon, M.I. (1999) Abnormal photoresponses and light-induced apoptosis in rods lacking rhodopsin kinase. Proc Natl Acad Sci U S A, 96, 3718-3722.

90 Roosing, S., Rohrschneider, K., Beryozkin, A., Sharon, D., Weisschuh, N., Staller, J., Kohl, S., Zelinger, L., Peters, T.A. and Neveling, K. (2013) Mutations in RAB28, encoding a farnesylated small GTPase, are associated with autosomal-recessive cone-rod dystrophy. The American Journal of Human Genetics, 93, 110-117.

91 Roosing, S., Thiadens, A.A., Hoyng, C.B., Klaver, C.C., den Hollander, A.I. and Cremers, F.P. (2014) Causes and consequences of inherited cone disorders. Progress in retinal and eye research, 42, 1-26.

92 Ávila-Fernández, A., Cantalapiedra, D., Aller, E., Vallespín, E., Aguirre-Lambán, J., Blanco-Kelly, F., Corton, M., Riveiro-Álvarez, R., Allikmets, R. and Trujillo-Tiebas, M.J. (2010) Mutation analysis of 272 Spanish families affected by autosomal recessive retinitis pigmentosa using a genotyping microarray. in press.

93 Armstrong, S.A., Brown, M.S., Goldstein, J.L. and Seabra, M.C. (1995) [5] Preparation of recombinant Rab geranylgeranyltransferase and Rab escort proteins. Methods in enzymology, 257, 30-41.

94 Pereira-Leal, J.B., Hume, A.N. and Seabra, M.C. (2001) Prenylation of Rab GTPases: molecular mechanisms and involvement in genetic disease. FEBS letters, 498, 197-200.

95 Lim, Y.S., Chua, C.E.L. and Tang, B.L. (2011) Rabs and other small GTPases in ciliary transport. Biology of the Cell, 103, 209-221.

96 Sanders, A.A.W.M. (2014) Identification and functional characterisation of new ciliary base proteins and investigation of diffusion kinetics across the ciliary transition zone in Caenorhabditis elegans roundworms. in press. 
97 Järvelä, I., Lehtovirta, M., Tikkanen, R., Kyttälä, A. and Jalanko, A. (1999) Defective intracellular transport of CLN3 is the molecular basis of Batten disease (JNCL). Human molecular genetics, 8, 1091-1098.

98 Katz, M.L., Gao, C.-L., Prabhakaram, M., Shibuya, H., Liu, P.-C. and Johnson, G.S. (1997) Immunochemical localization of the Batten disease (CLN3) protein in retina. Investigative ophthalmology \& visual science, 38, 2375-2386.

99 Lerner, T.J., Boustany, R.-M.N., Anderson, J.W., D'Arigo, K.L., Schlumpf, K., Buckler, A.J., Gusella, J.F. and Haines, J.L. (1995) Isolation of a novel gene underlying Batten disease, CLN3. Cell, 82, 949-957.

100 Seigel, G.M., Lotery, A., Kummer, A., Bernard, D.J., Greene, N.D., Turmaine, M., Derksen, T., Nussbaum, R.L., Davidson, B. and Wagner, J. (2002) Retinal pathology and function in a Cln3 knockout mouse model of juvenile Neuronal Ceroid Lipofuscinosis (batten disease). Molecular and Cellular Neuroscience, 19, 515-527.

101 Luiro, K., Kopra, O., Lehtovirta, M. and Jalanko, A. (2001) CLN3 protein is targeted to neuronal synapses but excluded from synaptic vesicles: new clues to Batten disease. Human molecular genetics, 10, 2123-2131.

102 Pereira-Leal, J.B., Hume, A.N. and Seabra, M.C. (2001) Prenylation of Rab GTPases: molecular mechanisms and involvement in genetic disease. FEBS Lett, 498, 197-200.

103 Petersen-Jones, S.M., Entz, D.D. and Sargan, D.R. (1999) cGMP phosphodiesterasealpha mutation causes progressive retinal atrophy in the Cardigan Welsh corgi dog. Invest Ophthalmol Vis Sci, 40, 1637-1644.

104 Suber, M.L., Pittler, S.J., Qin, N., Wright, G.C., Holcombe, V., Lee, R.H., Craft, C.M., Lolley, R.N., Baehr, W. and Hurwitz, R.L. (1993) Irish setter dogs affected with rod/cone dysplasia contain a nonsense mutation in the rod cGMP phosphodiesterase beta-subunit gene. Proc Natl Acad Sci U S A, 90, 3968-3972. 
Pggtlb-mediated protein geranylgeranylation is crucial for assembly of phosphodiesterase 6 and function of cone photoreceptor neurons

\author{
Nachiket D. Pendse ${ }^{\mathrm{a}}$, Martin O. Bergo ${ }^{\mathrm{b}}$, Stephen G. Young ${ }^{\mathrm{c}}$, and Visvanathan \\ Ramamurthy $^{\mathrm{a}, 1}$
}

aDepartments of Ophthalmology, Biochemistry and Biology, West Virginia University, Morgantown, WV; 'bahlgrenska Cancer Center, Gothenburg, Sweden; and ${ }^{\mathrm{c} D e p a r t m e n t s ~ o f ~}$ Medicine and Human Genetics, University of California, Los Angeles, CA

To whom correspondence should be addressed. E-mail: ramamurthyv@wvumedicine.org.

Key words: Prenylation, photoreceptor neurons, proteins-lipid modification, phosphodiesterase, protein assembly

Conflict of interests: The authors have no conflicts of interest

Manuscript information: Total 42 pages and 8 figures. Supporting information with 7 supplemental figures. 


\begin{abstract}
Multiple proteins in photoreceptor neurons undergo prenylation, a post-translational modification in which a farnesyl or a geranylgeranyl lipid group is added to a $\mathrm{C}$-terminal $C A A X$ motif. In cone photoreceptors, the catalytic subunit of phosphodiesterase 6 (PDE6) is thought to be geranylgeranylated. Multimeric PDE6 is required as an effector enzyme in the phototransduction cascade. However, it is not known whether lipid modification is important in cone PDE6 biosynthesis or whether prenylation is required in photoreceptors. To address these issues, we generated mice lacking Pggtlb (catalytic subunit of geranylgeranyl transferases) or Fntb (catalytic subunit of farnesyltransferases) in cone photoreceptor cells. Cone photoreceptors developed normally in the absence of Pggt1b and did not degenerate. However, at 1 month of age, cone function was abolished with drastic reduction in cone PDE6 levels, with impaired membrane anchorage. Moreover, cone PDE6 failed to assemble, because PDE6 could not to interact with its chaperone, aryl hydrocarbon receptor interacting protein like 1. Also reduced in Pggtlb-deficient mice were levels of other prenylated proteins, such as cone transducin, Gprotein-coupled receptor kinase, and nonprenylated retinal guanylate cyclase. In contrast, loss of farnesylation in cone photoreceptors did not result in any such defects, even at 6 months. We conclude that protein geranylgeranylation is required for cone-mediated vision.
\end{abstract}




\section{SIGNIFICANCE STATEMENT}

Here we show that cone photoreceptor function requires protein geranylgeranylation, as demonstrated by the absence of cone mediated visual response in mice lacking Pggtlb. The survival of cone photoreceptors did not depend on protein prenylation. The cone dysfunction was caused by the failure of a chaperone, aryl hydrocarbon receptor interacting protein like-1, to interact with catalytic subunits of cone PDE6, leading to defective assembly and instability of cone PDE6, the effector enzyme needed for phototransduction. Such defects were not observed in cones lacking functional farnesyltransferase. We conclude that protein geranylgeranylation is required for cone-mediated vision. 


\section{INTRODUCTION}

Prenylation is the addition of a lipid, either a farnesyl or a geranylgeranyl group, to the Cterminal cysteine of proteins with a $C A A X$ motif, where $C$ is cysteine, $A$ is an aliphatic amino acid, and $X$ is any amino acid (15). A farnesyl (C-15) group is added to the C-terminal cysteine, by farnesyl transferase (FTase) enzyme, if the amino acid residue in the $X$ position is preferentially methionine, serine, alanine, or glutamine. A geranylgeranyl (C-20) group is added to the C-terminal cysteine, by geranylgeranyl transferase (GGTase-I) enzyme, if the amino acid in the $X$ position is leucine or phenylalanine (14). After prenylation, the last three amino acid residues $(-A A X)$ are cleaved by the protease RAS-converting enzyme 1 and isoprenylcysteine methyltransferase catalyzes the addition of a methyl group to the newly exposed isoprenylcysteine in endoplasmic reticulum membranes $(15,49,59,106)$.

Although prenylated substrates are present in photoreceptor neurons, the role of prenylation in their development and function is not known. These highly specialized ciliated cells have elaborated membrane-enriched compartments, called outer segments (OS), containing the proteins needed for phototransduction. Several of these proteins including G-protein-coupled cone transducin- $\gamma$ (G $\gamma \mathrm{T} 2)$, G-protein-coupled rhodopsin kinase (GRK1), and phosphodiesterase 6 (PDE6) (36,107-111) undergo prenylation, which may be required for their anchorage, trafficking, and retention in the membranous disks in the OS.

Cone transducin, a heterotrimeric $\mathrm{G}$ protein consisting of a myristoylated $\mathrm{G} \alpha \mathrm{T} 2$ subunit, a G $\beta 3$ subunit, and a G $\gamma \mathrm{T} 2$ subunit, is thought to be farnesylated $(66,111,112)$. Previously, we showed that methyl esterification mediated by isoprenylcysteine methyltransferase is essential for the stability and membrane association of G $\gamma \mathrm{T} 2$ (59). Another farnesylated protein, GRK1, is present in mouse rods and cones. Cones in human contain GRK7, a homologue of GRK1 that is thought to be geranylgeranylated $(88,89)$. 
Cone PDE6, the crucial effector enzyme in phototransduction pathway, is composed of two identical catalytic subunits (PDE6 $\left.\alpha^{\prime}\right)$ that are thought to be geranylgeranylated $(12,109,113-115)$, and two inhibitory subunits (PDE6 $\left.\gamma^{\prime}\right)(41,66,116-118)$.The need for lipid modification in PDE6 is not clear. Recent structural studies suggest a need for prenylation in promoting PDE6 membrane interaction which in turn is important in optimizing the phototransduction cascade either by enhancing PDE6-transducin interaction or affecting the conformation of PDE6 (119). Based on our previous findings, we proposed that prenylation is likely needed in the earlier step of PDE6 biosynthesis, the folding and assembly of functional PDE6 in photoreceptor inner segments by a chaperone, aryl hydrocarbon receptor interacting protein like-1 (AIPL1) $(84,86,87,120)$.

In this study, we investigated the role of prenylation in cone photoreceptors. To identify cellular, molecular, and visual consequences of the lack of prenylation in cones, we used $\mathrm{Cre}$ loxP recombination in mice to eliminate $F n t b$ (encoding the catalytic subunit of FTase) or Pggtlb (encoding the catalytic subunit of GGTase-I). These experimental mice were generated by breeding $F n t b^{f l f l}$ or Pggtl $b^{f l f l}$ mice with mice expressing Cre recombinase driven by the conespecific HRGP promoter (121). 


\section{RESULT}

Generating Conditional Knockout Mice. Pggtlb or Fntb was eliminated by Cre-loxP recombination (Fig. S1). Pggt1b conditional mice were created by mating Pggt $1 b^{f l / w t}:$ HRGP-Cre males with $P g g t 1^{b f l f l}$ females to generate $P g g t 1 b^{f l f l}:$ HRGP-Cre mice (hereafter referred to as $P_{\text {ggtl }} b^{-/-}$mice $) . P g g t 1 b^{+/+}\left[\left(P g g t 1 b^{f l / w t}:\right.\right.$ HRGP-Cre, Pggtl $b^{f l f l}$, Pggt $\left.\left.1 b^{f l / w t}\right)\right]$ littermate controls were similar to C57BL/6J mice and had identical photoreceptor responses (not shown). A similar strategy was used to generate $F n t b$ conditional knockout mice $\left(F n t b^{--}\right)$and littermate controls $\left[\left(F n t b^{+/+}\left(F n t b^{f l / w t}:\right.\right.\right.$ HRGP-Cre, Fntb $\left.\left.b^{f l f l}, F n t b^{f l / w t}\right)\right]$.

In our animal models, excision of Pggtlb or Fntb in cone photoreceptors begins at postnatal day 4 (P4) (121). Cre was exclusively localized to the nucleus of cone photoreceptor cells, as shown by staining with polyclonal anti-Cre antibody and confirmed by double labeling with Cre and peanut agglutinin (Fig. S1 $C$ and $E$ ). Expression of Cre in $C r e$-positive mouse lines (Pggt $1 b^{-}$ ${ }^{/-}$and $\mathrm{Fntb}^{-{ }^{-}}$) was confirmed by western blotting (Fig. S1D).

Protein Geranylgeranylation Is Crucial for Cone Photoreceptor Function. To assess cone function, we used electroretinography (ERG). In ERG studies, the rod response was suppressed under saturating background light conditions, and the cone response (photopic ERGs) was assessed with flashes of light at increasing intensity. Cone-mediated responses were not altered at P16 in $\mathrm{Pggt}_{1 b^{-1-}}$ mice but were reduced by $90 \%$ at P25 and absent at P85 (both $P<0.02$ ) (Fig. 1A). The loss of photoreceptor function over time is shown in Fig. S2A.

Surprisingly, cone-mediated ERGs were similar between $F n t b^{-/}$and littermate controls, even at 6 months of age (P180) (Fig. $1 B$ and $\mathrm{S} 2 B$ ). Thus, Fntb-mediated protein farnesylation is not crucial for cone photoreceptor function. As expected, removal of Pggt1b or Fntb in cone 
photoreceptors did not affect rod function (Fig. S3). Altogether, our results highlight the need for Pggtlb-mediated protein geranylgeranylation in cone photoreceptor function.

\section{Survival of Cone Photoreceptors Is Unaffected in The Absence of Protein Prenylation. To} determine whether cone degeneration was responsible for the loss of cone function at P85 in $\operatorname{Pggtl}^{-/-}$mice, we checked the abundance and density of cone photoreceptor cells in retinal flat mounts. Staining for the cone OS marker, M-opsin, revealed no alteration in photoreceptor density in $\mathrm{Pggtl}^{-/-}$mice at P85 or in $\mathrm{Fntb}^{-/-}$mice at P180 (Fig. 1 C I and D I). In addition, we found no changes in the density of M-opsin positive photoreceptor cells in the dorsal, ventral central, nasal, and temporal regions of the retina (not shown). The morphology of cone photoreceptors appeared normal as shown by peanut agglutinin that stains the cone sheath in retinal cryosections (Fig. $1 C I I$ and $D I I$ ).

\section{Defective Protein Geranylgeranylation Leads to Reduction in The Levels of}

Phosphodiesterase and Transducin in Cone Photoreceptors. To determine whether the decreased photopic ERG response in cones lacking protein geranylgeranylation resulted from altered levels of prenylated phototransduction proteins, we immunoblotted retinal extracts from Pggtl $^{+/+}$and Pggtl $^{-/-}$mice at P85, when cone-mediated responses were absent. In Pggtlb ${ }^{-/}$ mice, the level of the catalytic subunit of cone PDE6 (PDE6 $\left.\alpha^{\prime}\right)$ was reduced by $90 \%(P=0.009)$, and the level of cone transducin ( $\alpha$ and $\gamma$ subunits) was reduced by $60 \%$ ( $P=0.001)$ (Fig. $2 A$ and $B)$. The reduction in cone transducin was surprising, since G $\gamma \mathrm{T} 2$ is thought to be farnesylated, and its interacting partner GaT2 is myristoylated. The levels of cone arrestin (cARR), a cone photoreceptor-specific protein that is not prenylated is not altered and served as a control. The levels of rod photoreceptor-specific proteins such as rod phosphodiesterase- $\beta$ (PDE6 $\beta$ ) subunit, transducin- $\alpha(\mathrm{G} \alpha \mathrm{T} 1)$, and rod arrestin (rARR) did not differ in $P_{g g t 1 b^{+/+}}$and Pggtl $^{-/-}$mice at P85. Thus, eliminating Pggtlb in cone photoreceptors does not affect protein levels in rod cells 
(Fig. $2 A$ and $B$ ). Strikingly, no changes occurred in the absence of protein farnesylation, including G $\gamma \mathrm{T} 2$, even at 6 months of age (Fig. S4 $A$ and $B$ ). In conclusion, the lack of Pggt $1 b$ mediated protein geranylgeranylation markedly reduces steady-state levels of cone PDE6 and transducin.

\section{Impaired Membrane Partitioning of Cone Phosphodiesterase and Transducin in The}

Absence of Pggt Ib. The addition of a lipid anchor to the $C A A X$ proteins is thought to increase hydrophobicity and provide firm membrane anchorage. To examine the effects of geranylgeranylation on the membrane attachment of phototransduction proteins, we performed isotonic cellular fractionation of retinal extracts from $\mathrm{Pggt}^{-/} \mathrm{b}^{-\alpha}$ mice at P30 when photopic ERG was reduced by $90 \%$ (Fig. 1) but cone PDE6 levels were reduced by only $60 \%$ (not shown). In $P_{\text {ggtl }} \mathrm{C}^{++}$littermate controls, $65 \%$ of cone PDE6 was in the membrane fraction $(P<0.05)$ versus only $20 \%$ in $P g g t 1 b^{--}$mice (Fig. $3 A$ and B). The membrane association of G $\gamma \mathrm{T} 2$ was also defective (Fig. $3 A$ and $B$ ). Deficiency in Pggt 1 -mediated protein geranylgeranylation did not affect the levels of guanylate cyclase-1 (RetGC1, a transmembrane protein used as a membrane-bound control) or AIPL1 (cytosolic protein control) (Fig.3 A). As expected, the membrane attachment of the rod PDE6 subunits, GRK1, and cone arrestin were unaffected. The membrane partitioning of several prenylated retinal proteins was unaltered in $\mathrm{Fntb}^{-/}$mice (Fig. S5).

Next, to determine whether prenylation of PDE6 and transducin is defective in Pggt1 ${ }^{-/}$

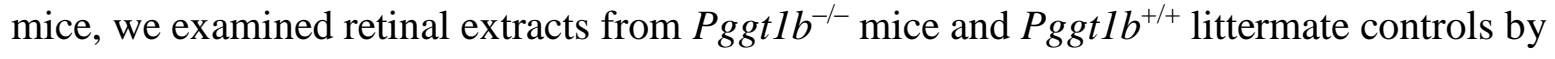
Triton-X-114 phase partitioning (122), which segregates prenylated and membrane proteins into the detergent phase and cytosolic proteins into the aqueous phase. Cone PDE6 was equally distributed between the two phases in the controls but was mostly in the aqueous phase in $P_{g g t 1} b^{--}$mice $(94 \%, P<0.02)$. Moreover, in $P g g t 1 b^{--}$mice, $55 \%$ of G $\gamma \mathrm{T} 2$, a protein thought to be farnesylated, was in the aqueous phase $(P<0.02)$ (Fig. $4 A$ and $B$ ), whereas in the controls, 
$96 \%$ of G $\gamma \mathrm{T} 2$ was in the detergent fraction. No defects in PDE6 and G $\gamma$ T2 were found in Fntb mice. These results show that Pggtlb-mediated protein geranylgeranylation is required for proper membrane attachment of cone PDE6 and G $\gamma$ T2.

\section{Pggt1b-mediated Protein Geranylgeranylation Is Important for The Assembly of Cone}

Phosphodiesterase. Our previous work on AIPL1 showed a link between prenylation, protein stability, and the assembly of heteromeric cone PDE6 $(84,86,106)$. To investigate whether the lack of prenylation in our animal models affects the assembly of heteromeric cone PDE6, we did immunoprecipitation studies. Proteins were immunoprecipitated with ROS-I, a monoclonal antibody that recognizes the assembled heteromeric cone PDE6, separated on SDS-PAGE gels, and immunoblotted with antibodies specific for cone PDE6 $(84,123)$. As expected, assembled PDE6 was present in the controls (Fig. 5 A, top panel) but not in Pggtl P $^{--}$mice, indicating defective assembly (Fig. $5 A$ and $B$ ). The absence of Fntb did not affect cone PDE6 assembly (Fig. 5 A, bottom panels).

\section{Interaction of AIPL1 with Cone Phosphodiesterase Requires Protein Geranylgeranylation.} In our previous studies, we showed that the lipid moiety in PDE6 was crucial for its interaction with AIPL1 $(84,86)$. To investigate whether AIPL1 interacts with cone PDE6 in the absence of Pggtlb or Fntb, we performed immunoprecipitation experiments with monoclonal antibody against AIPL1 (84). Cone PDE6 and AIPL1 co-immunoprecipitated in Pggtlb ${ }^{+/+}$controls but not in $P_{g g t 1 b^{-/}}$mice (Fig. 6, $P<0.05$ ). No such defects were detected in the absence of protein farnesylation (not shown).

\section{Rhodopsin kinase (GRK1) And Retinal Guanylate Cyclase (RetGC1) Are Absent in Cones Lacking Pggt1b. GRK1, a farnesylated protein, and RetGC1, a nonprenylated protein, are normally expressed in rod and cone photoreceptor cells. However, we could not analyze these proteins in whole retinal lysates, as prenylation is eliminated only in cone photoreceptor cells in}


our models. Therefore, we examined retinal cryosections by immunohistochemistry with antibodies specific to GRK1 and RetGC1 (Fig. 7) Both GRK1 and RetGC1 were present in rod and cone photoreceptors in Pggt $1 b^{+/+}$controls but were not detected in cone photoreceptors of Pggtl $b^{--}$mice, as shown by staining with peanut agglutinin (Fig. $7 A$ and $B$ ). We also counted

the number of PNA-positive cones that stain for GRK1 and RetGC1. In Pggtl $b^{--}$mice, 99\% of cone photoreceptors did not express GRK1 or RetGC1 (Fig. $7 D, P<0.02$ ). The expression of cone opsin was unaffected (Fig. 7 C). Expression of GRK1, M-opsin, and RetGC1 was not defective in Fntb mice (Fig. S6 and data not shown). These results highlight importance of protein geranylgeranylation for expression of GRK1 and RetGC1 in cones.

\section{Normal Trafficking of Phosphodiesterase to The Cone OS in The Absence of Protein}

Geranylgeranylation. The OS is thought to be the default location for PDE6 $(124,125)$. To determine whether prenylation participates in the trafficking of PDE6 to the cone OS, we examined the localization of PDE6 in retina lacking Pggtlb or Fntb. In Pggt1 $b^{-1-}$ mice, cone PDE6 was present in the OS and it co-localized with M-opsin, an OS marker (Fig. 8 A). Cones expressing PDE6 in the OS showed robust expression of $\mathrm{Cre}$ recombinase, suggesting efficient elimination of $P$ ggtlb (Fig. 8 B). Additionally, Triton-X-114 results performed from retinal extracts at the same age as our localization study support defective prenylation of cone PDE6 in Pggtlb animals (Fig. 4). Overall our results show that protein geranylgeranylation is not needed for trafficking of cone PDE6 and transducin to the cone OS (Fig. S7). 


\section{DISCUSSION}

This study shows that Pggtlb-mediated protein geranylgeranylation is required for conemediated vision. In $\mathrm{Pggtlb}^{-/}$mice, photopic ERGs were drastically reduced, demonstrating that cone photoreceptor function requires Pggtlb-mediated protein geranylgeranylation. However, the survival of cone photoreceptors did not depend on protein prenylation. The primary cause of cone dysfunction was the failure of AIPL1 to interact with catalytic subunits of cone PDE6, leading to defective assembly and instability of cone PDE6. Such defects were not found in cones lacking functional farnesyl transferase, highlighting the crucial role of Pggtlb-mediated protein geranylgeranylation in cone photoreceptors.

We attribute the absence of light-evoked cone responses in $\mathrm{Pggtlb}^{-/}$mice to the loss of cone PDE6, the key effector enzyme in the cone phototransduction pathway $(41,116,117)$. In Pggtl $b^{-}$ ${ }^{\prime}$ mice, Triton X-114 fractionation experiments showed loss of lipid modification in PDE6 catalytic subunits, and the defective geranylgeranylation was confirmed by the reduced membrane association of PDE6. In contrast, cone PDE6 associated normally with membranes in $\mathrm{Fntb}^{--}$mice. These results agree with the pattern of geranylgeranyl modification predicted from the $C A A X$ motif, $C L M L$, in cone PDE6 catalytic subunits $(12,115)$.

In our previous studies, PDE6 subunits were synthesized normally in the absence of AIPL1 but were unstable and failed to assemble properly $(84,86,87)$. Similarly, in the absence of protein geranylgeranylation in $\mathrm{Pggtlb}^{--}$mice, AIPL1 did not interact with cone PDE6 subunits, leading to its defective assembly. Misassembled PDE6 is likely degraded by proteasomes in the inner segment of photoreceptors culminating in decreased levels of PDE6 $(87,120)$. The need behind the lipid modification in PDE6 assembly is not known. It is possible that lipid anchors in cone PDE6 could potentially direct it to the endoplasmic reticulum, where AIPL1 may participate in its folding and assembly. However, it is unclear whether AIPL1 is cytosolic or bound to the endoplasmic reticulum membrane. Alternatively, the geranylgeranyl lipid anchors on cone PDE6 
may act as a "molecular grip" to facilitate the interaction of AIPL1 with catalytic subunits of PDE6 in the cytosol and thereby promote its assembly with inhibitory subunits $\left(\gamma^{\prime}\right)$, a step needed for the synthesis of functional PDE6. Although our studies show that prenylation is needed for initial folding and assembly of PDE6 heteromer, present investigation does not discount the possibility that prenylation may also play a role in optimizing the phototransduction cascade as suggested earlier (119).

Organized trafficking of newly synthesized phototransduction components from the inner segment to the OS is essential for efficient phototransduction (1). Lipid anchors are thought to be needed to target peripheral membrane proteins to the OS $(1,126)$. Surprisingly, in the absence of protein geranylgeranylation, PDE6 catalytic subunits localized to the cone OS. We believe that the N-terminal GAF domain of PDE6 has a targeting motif that helps direct it to the OS (124). Cross-prenylation is also possible in our mouse model, since FTase and GGTase have similar substrate preferences. However, in Triton X-114 fractionation studies, cone PDE6 in Pggtl $b^{-/}$ mice partitioned to the aqueous phase, demonstrating the absence of any lipid anchors.

Cone-mediated ERGs in Fntb mice were unaffected even at 6 months. This was unexpected because farnesyltransferases in cone photoreceptors are thought, based on $C A A X$ sequence prediction, to have multiple substrates, including GRK1 and G $\gamma \mathrm{T} 2$. Thus, protein farnesylation should have a greater effect on cone function. However, the absence of detrimental effects after removal of FTase in cone cells is consistent with reports that FTase is dispensable in adult tissues (33). The notion that FTase is not essential for mammalian tissues is consistent with studies of farnesyl transferase inhibitors, which have fewer adverse side effects (127). Intriguingly, along with cone PDE6, membrane attachment and Triton X-114 partitioning of cone G $\gamma \mathrm{T} 2$ were altered in $P g g t 1 b^{-/}$mice but not in Fntb mice. Although the CAAX sequence (CVIS) of G $\gamma \mathrm{T} 2$ suggests that it is farnesylated (111), our evidence suggests that it is a substrate of GGTase-I and is likely geranylgeranylated. 
In addition to PDE6 and transducin, RetGC1 and GRK1 levels were also reduced in cones lacking Pggt 1b. RetGC1 is a nonprenylated transmembrane protein, and GRK1 is thought to be farnesylated. It is unclear why RetGC1 and GRK1 levels were reduced. An early study speculated that multiple proteins destined for the cone OS are co-transported in vesicles (128). We suspect that a small GTP protein such as geranylgeranylated Rab is involved in the vesicular trafficking of OS-resident proteins in cones. Defects in the lipid modification of this yet-to-beidentified protein impair vesicular trafficking, leading to the accumulation and proteolytic degradation of RetGC1 and GRK1 in cones.

In conclusion, our findings show that geranylgeranylation of PDE6 is essential for its interaction with its chaperone AIPL1, as well as its subunit assembly, membrane anchorage, and stability but not for the movement of PDE6 to the cone OS. The failure of cone PDE6 to assemble is the underlying cause of cone photoreceptor dysfunction. 


\section{MATERIAL AND METHODS}

Mouse Models. HRGP-Cre mice were from Dr. Yun-Zheng Le (University of Oklahoma Health

Science Center). Both males and females were used in the study. Mouse experiments were done in accordance with the National Institutes of Health guidelines. The protocol was approved by Institutional Animal Care and Use Committee of West Virginia University. A detailed description of all other methods appears in SI Materials and Methods. 


\section{ACKNOWLEDGEMENTS}

We thank Thamarai Saravanan for maintaining animal stocks and Dr. Karen Martin for advice and the use of the West Virginia University Microscopic Imaging Facility. We thank Dr. Yun Zheng Le from University of Oklahoma Health Sciences Center for sharing HRGP-Cre mice. We also thank Dr. Nidhi Saraiya and members of the Ramamurthy laboratory for constructive criticism. This work was supported by National Institutes of Health Grants R01EY017035 (to V.R.) and R01HL126551 (to S.Y.), West Virginia Lions, and an Unrestricted Research to Prevent Blindness Challenge Grant (West Virginia University).

\section{AUTHOR CONTRIBUTIONS}

N.D.P. and V.R. designed the research and wrote the manuscript; N.D.P. performed the research; N.D.P. and V.R. analyzed the data; M.O.B. and S.Y. provided advice and reagents and helped write the manuscript. 


\section{REFERENCES}

1. Zhang FL \& Casey PJ (1996) Protein prenylation: molecular mechanisms and functional consequences. Annual review of biochemistry 65(1):241-269.

2. Hartman HL, Hicks KA, \& Fierke CA (2005) Peptide specificity of protein prenyltransferases is determined mainly by reactivity rather than binding affinity. Biochemistry 44(46):15314-15324.

3. Anderson JL \& Hrycyna CA (2006) 9 Structure and function of isoprenylcysteine carboxylmethyltransferase (Icmt): A key enzyme in CaaX processing. The Enzymes 24:245-272.

4. Christiansen JR, Kolandaivelu S, Bergo MO, \& Ramamurthy V (2011) RAS-converting enzyme 1-mediated endoproteolysis is required for trafficking of rod phosphodiesterase 6 to photoreceptor outer segments. Proceedings of the National Academy of Sciences 108(21):8862-8866.

5. Christiansen JR, et al. (2016) Deficiency of Isoprenylcysteine Carboxyl Methyltransferase (ICMT) Leads to Progressive Loss of Photoreceptor Function. The Journal of Neuroscience 36(18):5107-5114.

6. Qin N \& Baehr W (1994) Expression and mutagenesis of mouse rod photoreceptor cGMP phosphodiesterase. Journal of Biological Chemistry 269(5):3265-3271.

7. Ong OC, Ota IM, Clarke S, \& Fung B (1989) The membrane binding domain of rod cGMP phosphodiesterase is posttranslationally modified by methyl esterification at a Cterminal cysteine. Proceedings of the National Academy of Sciences 86(23):9238-9242.

8. Fukada Y, et al. (1990) Farnesylated gamma-subunit of photoreceptor G protein indispensable for GTP-binding. Nature 346(6285):658-660.

9. Anant J, et al. (1992) In vivo differential prenylation of retinal cyclic GMP phosphodiesterase catalytic subunits. Journal of Biological Chemistry 267(2):687-690.

10. Inglese J, Koch WJ, Caron MG, \& Lefkowitz RJ (1992) Isoprenylation in regulation of signal transduction by G-protein-coupled receptor kinases. Nature 359(6391):147-150.

11. Akhmedov NB, et al. (1998) Canine cone transducin-gamma gene and cone degeneration in the cd dog. Investigative ophthalmology \& visual science 39(10):1775-1781.

12. Bigay J, Faurobert E, Franco M, \& Chabre M (1994) Roles of lipid modifications of transducin subunits in their GDP-dependent association and membrane binding. Biochemistry 33(47):14081-14090.

13. Burns ME \& Arshavsky VY (2005) Beyond counting photons: trials and trends in vertebrate visual transduction. Neuron 48(3):387-401.

14. Weiss ER, et al. (2001) Species-specific differences in expression of G-protein-coupled receptor kinase (GRK) 7 and GRK1 in mammalian cone photoreceptor cells: implications for cone cell phototransduction. The Journal of Neuroscience 21(23):9175-9184.

15. Horner TJ, Osawa S, Schaller MD, \& Weiss ER (2005) Phosphorylation of GRK1 and GRK7 by cAMP-dependent protein kinase attenuates their enzymatic activities. Journal of Biological Chemistry 280(31):28241-28250.

16. Wensel TG (2008) Signal transducing membrane complexes of photoreceptor outer segments. Vision research 48(20):2052-2061.

17. Cote R (2004) Characteristics of photoreceptor PDE (PDE6): similarities and differences to PDE5. International journal of impotence research 16:S28-S33. 
18. Li T, Volpp K, \& Applebury ML (1990) Bovine cone photoreceptor cGMP phosphodiesterase structure deduced from a cDNA clone. Proceedings of the National Academy of Sciences 87(1):293-297.

19. Baehr W (2014) Membrane Protein Transport in Photoreceptors: The Function of PDE 8 The Proctor Lecture. Investigative ophthalmology \& visual science 55(12):86538666.

20. Baehr W, Devlin MJ, \& Applebury ML (1979) Isolation and characterization of cGMP phosphodiesterase from bovine rod outer segments. Journal of Biological Chemistry 254(22):11669-11677.

21. Deterre P, Bigay J, Forquet F, Robert M, \& Chabre M (1988) cGMP phosphodiesterase of retinal rods is regulated by two inhibitory subunits. Proceedings of the National Academy of Sciences 85(8):2424-2428.

22. Gillespie PG \& Beavo JA (1988) Characterization of a bovine cone photoreceptor phosphodiesterase purified by cyclic GMP-sepharose chromatography. Journal of Biological Chemistry 263(17):8133-8141.

23. Hamilton SE \& Hurley JB (1990) A phosphodiesterase inhibitor specific to a subset of bovine retinal cones. Journal of Biological Chemistry 265(19):11259-11264.

24. Zhang Z, et al. (2015) Domain organization and conformational plasticity of the G protein effector, PDE6. Journal of Biological Chemistry 290(20):12833-12843.

25. Kirschman LT, et al. (2009) The Leber congenital amaurosis protein, AIPL1, is needed for the viability and functioning of cone photoreceptor cells. Human Molecular genetics (2010) 19 (6): 1076-1087.

26. Kolandaivelu S, Huang J, Hurley JB, \& Ramamurthy V (2009) AIPL1, a protein associated with childhood blindness, interacts with $\alpha$-subunit of rod phosphodiesterase (PDE6) and is essential for its proper assembly. Journal of Biological Chemistry 284(45):30853-30861.

27. Kolandaivelu S, Singh RK, \& Ramamurthy V (2013) AIPL1, A protein linked to blindness, is essential for the stability of enzymes mediating cGMP metabolism in cone photoreceptor cells. Human molecular genetics:ddt496.

28. Kolandaivelu S, Chang B, \& Ramamurthy V (2011) Rod phosphodiesterase-6 (PDE6) catalytic subunits restore cone function in a mouse model lacking cone PDE6 catalytic subunit. Journal of Biological Chemistry 286(38):33252-33259.

29. Le Y-Z, et al. (2004) Targeted expression of Cre recombinase to cone photoreceptors in transgenic mice. Mol Vis 10:1011-1018.

30. Justice JM, Murtagh JJ, Moss J, \& Vaughan M (1995) Hydrophobicity and subunit interactions of rod outer segment proteins investigated using Triton X-114 phase partitioning. Journal of Biological Chemistry 270(30):17970-17976.

31. Hurwitz RL, Bunt-Milam AH, \& Beavo JA (1984) Immunologic characterization of the photoreceptor outer segment cyclic GMP phosphodiesterase. Journal of Biological Chemistry 259(13):8612-8618.

32. Cheguru P, Zhang Z, \& Artemyev NO (2014) The GAFa domain of phosphodiesterase- 6 contains a rod outer segment localization signal. Journal of neurochemistry 129(2):256263.

33. Muradov H, Boyd KK, \& Artemyev NO (2010) Rod phosphodiesterase-6 PDE6A and PDE6B subunits are enzymatically equivalent. Journal of Biological Chemistry 285(51):39828-39834. 
34. Pearring JN, Salinas RY, Baker SA, \& Arshavsky VY (2013) Protein sorting, targeting and trafficking in photoreceptor cells. Progress in retinal and eye research 36:24-51.

35. Baker SA, et al. (2008) The outer segment serves as a default destination for the trafficking of membrane proteins in photoreceptors. The Journal of cell biology 183(3):485-498.

36. Mijimolle N, et al. (2005) Protein farnesyltransferase in embryogenesis, adult homeostasis, and tumor development. Cancer cell 7(4):313-324.

37. Santucci R, Mackley PA, Sebti S, \& Alsina M (2003) Farnesyltransferase inhibitors and their role in the treatment of multiple myeloma. Cancer Control 10(5):384-387.

38. Karan S, Zhang H, Li S, Frederick JM, \& Baehr W (2008) A model for transport of membrane-associated phototransduction polypeptides in rod and cone photoreceptor inner segments. Vision research 48(3):442-452. 


\section{FIGURE LEGENDS}

Fig. 1. Protein geranylgeranylation is crucial for cone photoreceptor function. ( $A$ and $B$ ) Representative waveforms of cone ERG responses recorded from mice lacking Pggtlb (A) and Fntb $(B)$ with their littermate controls at indicated ages $(n=3)$. ( $C$ and $D)$ Survival of cone photoreceptors is unaffected in the absence of protein prenylation. (C panel I) Representative flat-mounts of retinas from Pggtlb $^{-/}($right $)$and Pggtlb $^{+/+}$mice (left) littermate control at P85 stained for M-opsin (green). Scale bar, $50 \mu \mathrm{M}$. (C panel II) Retinal cross sections from P85 mice Pggtlb $^{-/-}\left(\right.$right) and Pggt $1 b^{+/+}($left $)$littermate controls, labeled with peanut agglutinin (PNA, red) and DAPI (4',6-diamidino-2-phenylindole, blue). Scale bar, $10 \mu \mathrm{M}$. PNA stains cone sheath and DAPI staining marks nuclei. (D panel $I$ ) Representative flat-mounts of retinas from $F n t b^{-1-}$ (right) and $\mathrm{Fntb}^{+/+}$mice (left) littermate controls at P180 stained for M-opsin (green). Scale bar, $50 \mu \mathrm{M}$. (D panel II) Retinal cross sections from P180 Fntb ${ }^{-/}$(right) and $\mathrm{Fntb}^{+/+}$mice (left) littermate controls littermate controls, labeled with PNA (red) and DAPI (blue). Scale, $10 \mu$ M. $n$ = 3. OS, outer segment; IS, inner segment; ONL, outer nuclear layer.

Fig. 2. Defective protein geranylgeranylation leads to reduction in the levels of phosphodiesterase and transducin in cone photoreceptors. (A) Representative immunoblots of retinal protein extracts from $\mathrm{Pggtl}^{-/-}$mice and $\mathrm{Pggtl}^{+/+}$littermate controls at P85 probed with antibodies against the indicated proteins. (B) Quantitation of results derived from panel A. Integrated intensity values were normalized to $\alpha$-tubulin. $n=4,{ }^{*} P<0.001$ ( $t$ test). NS, not significant.

Fig. 3. Impaired membrane partitioning of cone phosphodiesterase and transducin in the absence of Pggtlb. (A) Isotonic cellular fractionation of P30 retinal lysates from $P_{g g t 1 b^{-1}}$ mice and 


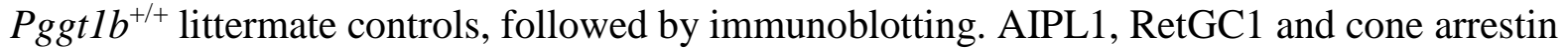
(cARR) served as controls $(n=4)$. (B) Quantitation of western blots from panel $A . n=4, * * P<$ 0.05; T, Total; C, cytosolic fraction (red bar); M, Membrane fraction (black bar); +/+ = $\operatorname{Pggtlb}^{+/+} ;-/-=\operatorname{Pggtlb}^{-/-}$

Fig. 4. Inefficient partitioning of cone phosphodiesterase and cone transducin- $\gamma$ subunit in Triton X-114 detergent in the absence of Pggt1b. (A) Triton X-114 phase partitioning of P30 retinal lysates from $\mathrm{Pggtlb}^{-/-}$and $\mathrm{Fntb}^{-/-}$mice and their respective $\mathrm{Pggtlb}^{+/+}$and $\mathrm{Fntb}^{+/+}$littermate controls, followed by immunoblotting. (B) Quantitation of western blots from panel $A$ (for Pggtlb samples). $n=2$ (four retinae from each genotype in each sample), $* * P<0.05 .+/+=$

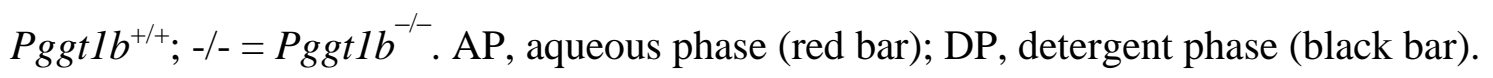

Fig. 5. Pggtlb-mediated protein geranylgeranylation is important for the assembly of cone phosphodiesterase. (A) At P32, cone PDE6 subunit in retinal extracts of Pggtlb and Fntb littermate mice were immunoprecipitated with monoclonal ROS-1 antibody, and immunoblots were probed with antibodies specific for cone PDE6. Nonspecific mouse IgG was used as control. (B) Quantitation of western blots from $A$. Integrated intensity values were determined with Odyssey imaging software $(n=4, * * P<0.02) .+/+=$ Pggtl $^{+/+} ;-/-=$Pggtl $^{-/-}$. T, Total; $\mathrm{U}$, Unbound fraction (red bar) and B, Bound fraction (black bar).

Fig. 6. Interaction of AIPL1 with cone phosphodiesterase requires protein geranylgeranylation.

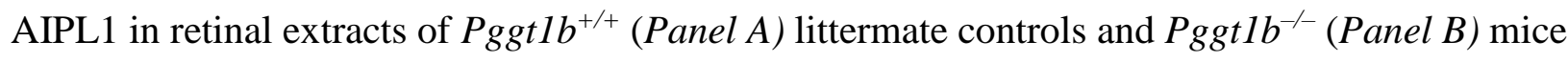
at P28 was immunoprecipitated with a monoclonal antibody (B-6 AIPL1), and immunoblots were probed with antibodies specific for catalytic subunit of cone PDE6 or AIPL1 $(n=4, * * P<$ $0.05)$.

Fig. 7. Rhodopsin kinase (GRK1) and Retinal guanylate cyclase (RetGC1) are absent in cones

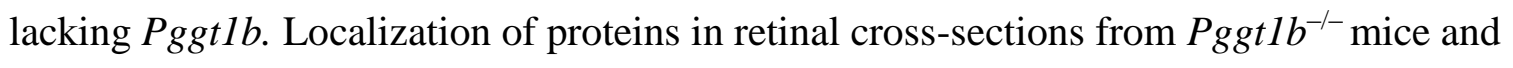


Pggt $1 b^{+/+}$littermate control at P85. (A) GRK1 (green) co-localizes with PNA (red) in Pggt1 $b^{+/+}$ (top panel A). (B) RetGC1 (green) co-localizes with PNA (red) in Pggtlb ${ }^{+/+}$(top panel B). Both RetGC1 and GRK1 are absent, in cones, in Pggt1b ${ }^{-/-}$at P85 (bottom panels in A and B). (C) Mopsin (green) co-localizes with PNA (red) in Pggt $1 b^{-/}$and littermate controls. (D) Quantification of relative number of cone photoreceptors expressing GRK1 and RetGC1. $n=3, * P<0.02$. Scale bar,10 $\mu \mathrm{M} .+/+=$ Pggt $1 b^{+/+} ;-/-=P g g t 1 b^{-/}$, Nuclei are stained with DAPI (blue). Cones expressing GRK1 (black bar); Cones expressing RetGC1 (grey bar)

Fig. 8. Normal trafficking of phosphodiesterase to the cone OS in the absence of protein geranylgeranylation. Localization of cone PDE6 in retinal cross-sections from P30 Pggt $1 b^{-/-}$ mice. (A) M-opsin (green) co-localizes with PDE6 $\alpha^{\prime}$ (red) in the cone OS. $(B)$ Cre positive cells (green) express PDE6 $\alpha^{\prime}$ (red) in the cone OS. OS, outer segment; IS, inner segments; ONL, outer nuclear layer. Scale bar, $10 \mu \mathrm{M}$. 


\section{SUPPORTING INFORMATION}

\section{Supplementary Material and Methods}

Electroretinography (ERG). ERG was performed as described (59). Littermates were darkadapted overnight, and the eyes were dilated (with phenylephrine and tropicamide, 1:1) for 10 min. Mice were placed on a $37^{\circ} \mathrm{C}$ platform, and isoflurane anesthesia (1.5\% in $2.5 \%$ oxygen) was given through a nose cone. A reference electrode was placed subcutaneously in the scalp, and silver wire electrodes were positioned above the cornea; contact was made with methylcellulose solution. Light flashes were presented by placing the mouse in a Ganzfield apparatus. Corneal evoked potentials were collected with UTAS-E4000 Visual Electrodiagnostic Test System and EMWIN 8.1.1 software (LKC Technologies). Background light $\left(30 \mathrm{~cd} \cdot \mathrm{m}^{-2}\right)$ was presented for $10 \mathrm{~min}$, and flicker responses were recorded in the presence of the background light. Representative waveforms are shown.

Immunoblotting. Flash-frozen retinal samples in $1 \times$ PBS containing Pierce protease inhibitor (Thermo Scientific) were solubilized by sonication with four 20 -msec pulses at power setting 6 (Misonix XL-2000). The protein concentration was estimated with a NanoDrop spectrophotometer (Thermo Scientific). Protein samples $(150 \mu \mathrm{g})$ were seperated on 4-20\% polyacrylamide gels (Criterion, Bio-Rad). Proteins were transferred to Immobilon-FL membranes (Millipore) and incubated with the following primary antibodies: anti-PDE6 $\alpha$ (Cytosignal), anti-PDE6 $\beta$ (Thermo Fisher), anti-G $\gamma$ T1 (Santa Cruz Biotechnology), anti-G $\gamma \mathrm{T} 2$ (gift from Dr. Vadim Arshavsky, Duke University Eye Center, Durham, NC), anti-GRK1

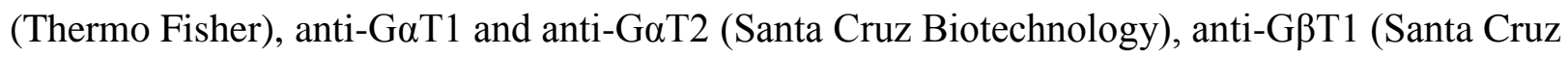
Biotechnology), anti-arrestin, anti-RetGC1 (Affinity Bioreagents), and anti-PDE6 $\alpha^{\prime}$ (3184p$(84,86,120)$. The primary antibodies were detected with Odyssey goat anti-rabbit Alexa 680 and Odyssey goat anti-mouse Alexa 680 secondary antibodies (Li-Cor Biosciences; 1:50,000). 
Membranes were scanned with an Odyssey Infrared Imaging System (Li-Cor Biosciences). Representative images from three or more independent experiments are shown.

Immunoprecipitation. Cone PDE6 assembly was assessed by immunoprecipitation with ROS-1 monoclonal antibody as described $(84,86,106)$. Retinas in Eppendorf tubes containing $1 \times$ PBS and Pierce protease inhibitors (Thermo Scientific) were placed on ice and briefly homogenized by sonication with five 15-msec pulses at power setting 6 (Misonix XL-2000). Triton X-100 $(0.1 \%)$ was added to aid protein solubilization, and samples were nutated on ice for 30 min at $4^{\circ} \mathrm{C}$. After centrifugation at $5000 \times g$ for $5 \mathrm{~min}$ at $4^{\circ} \mathrm{C}$, the supernatant was collected and cellular debris was discarded. The supernatant was incubated with ROS-1-coupled protein A/G beads (UltraLink® Immobilized protein A/G, PIERCE) for $3 \mathrm{~h}$. Unbound proteins were removed, and the beads were washed with Triton buffer (1\% Triton X-100, $50 \mathrm{mM}$ Tris, $\mathrm{pH} 7.5,300 \mathrm{mM}$ $\mathrm{NaCl}, 5 \mathrm{mM}$ EDTA, and $0.02 \% \mathrm{NaN}_{3}$ ). Bound PDE6 was eluted from the beads by adding SDSPAGE sample buffer (62.5 mM Tris, $\mathrm{pH}$ 6.8, 2\% SDS, 10\% glycerol, $0.005 \%$ bromophenol blue, and 5\% 2-mercaptoethanol) and boiling for $5 \mathrm{~min}$. Eluates were resolved on polyacrylamide gels and immunoblotted as described above. The interaction of cone PDE6 with AIPL1 was similarly assessed by immunoprecipitation with B6 AIPL1 monoclonal antibody linked to protein A/G beads.

Triton X-114 Phase Partitioning. TritonX-114 phase partitioning was done as described (122). Briefly, flash-frozen retinal samples were homogenized in buffer C (20 mM Tris-HCL, pH 7.5, $0.5 \mathrm{mM} \mathrm{MgCl}_{2}, 0.05 \mathrm{mM}$ EGTA, $0.5 \mathrm{mM} \mathrm{NaN}_{3}$, and $150 \mathrm{mM} \mathrm{NaCl}$ ) by sonication with five 15-msec pulses at power setting 6 (Misonix XL-2000). After centrifugation at $5000 \mathrm{~g}$ for $5 \mathrm{~min}$ at $4{ }^{\circ} \mathrm{C}$, the supernatant was collected, and cellular debris was discarded. Triton X-114 (final concentration $1 \%$ ) was added to the supernatant, gently mixed, and kept on ice for 5-10 min. After warming at $37^{\circ} \mathrm{C}$ for $5-10 \mathrm{~min}$, the samples were centrifuged at $300 \mathrm{~g}$ for $5 \mathrm{~min}$ at $37^{\circ} \mathrm{C}$ in 
a swinging bucket rotor pre-warmed to $37^{\circ} \mathrm{C}$. After centrifugation, the upper aqueous phase was aspirated, and $1 \%$ Triton $\mathrm{X}-114$ was added to final volume of $100 \mu \mathrm{l}$. To the detergent phase, buffer $\mathrm{C}$ was added to match the volume of the aqueous phase. The proteins were precipitated overnight using Trichloroacetic acid (TCA)/Acetone in the ratio of 1:8:1 (TCA: acetone: retinal extract). The precipitated samples were washed with $1 \mathrm{ml}$ ice-cold acetone, centrifuged at 25,000 rpm for 15 mins at $4^{\circ} \mathrm{C}$ and vacuum dried. The pellets were dissolved in equal volume of buffer C (122). Equal volumes of aqueous and detergent phase samples were analyzed by SDS-PAGE followed by immunoblotting as described above.

Membrane fractionation. Cells were fractionated as described $(86,106)$. Briefly, flash-frozen retinal samples were homogenized in $1 \times$ PBS containing Pierce protease inhibitors (Pierce protease inhibitor, Thermo Scientific) by sonication with five 15 -msec pulses at power setting 6 (Misonix XL-2000). After centrifugation at $5000 \mathrm{~g}$ for $5 \mathrm{~min}$ at $4^{\circ} \mathrm{C}$, the supernatant (total fraction) was collected, and cellular debris was discarded. The supernatant was spun at 45,000 $g$ for $30 \mathrm{~min}$ in TLA-55 rotor (Beckman Coulter) to isolate the soluble fraction. The high-speed supernatant (cytosol soluble fraction) was removed, and the pellet (membrane fraction) was resuspended in an equal volume of $1 \times$ PBS. All protein samples were then analyzed by SDSPAGE followed by immunoblotting to check for the distribution of cytosolic and membrane proteins.

Retinal flat mounts. Retinal flat mounts were performed as described $(84,86,106)$. Whole eyes were enucleated, and the dorsal side was marked and eyes were fixed in $4 \%$ paraformaldehyde in $1 \times$ PBS for $30 \mathrm{~min}$, and the cornea and lens were dissected carefully to preserving the known orientation. The retinas were isolated from the retinal pigment epithelium and placed $4 \%$ paraformaldehyde for $6 \mathrm{~h}$. For immunocytochemistry, the tissue was washed with $1 \times$ PBS three times for 30 min each, and nonspecific binding sites were blocked by incubating with blocking 
buffer for $4 \mathrm{~h}$. The retinas were incubated for $12 \mathrm{~h}$ with primary antibodies $(1: 1000$ in $1 \times \mathrm{PBS}$ with $0.01 \%$ Triton $\mathrm{X}-100$. Whole retinal tissues were washed twice in $1 \times$ PBS solution with $0.01 \%$ Triton $\mathrm{X}-100$ for $30 \mathrm{~min}$ each and $1 \times$ PBS for $45 \mathrm{~min}$ and incubated overnight with Odyssey goat anti-rabbit Alexa 488 IgG secondary antibody (1:1000, Li-Cor Biosciences). Secondary antibody was removed, and the retinae were washed as described above and placed on Superfrost Plus slides (Fisher Scientific) with the photoreceptor cells outer segment facing down. Radial cut was made to flatten the concave tissue and to divide the tissue into four quadrants: dorsal-rostral, dorsal-caudal, ventral-rostral and ventral-caudal. Finally, the whole retinal tissue was flat mounted, vitreal side up, on the slide and was cover slipped for imaging.

Immunohistochemistry. Immunohistochemistry was done as described (59). Mice were euthanized by $\mathrm{CO}_{2}$ inhalation, and the eyes were enucleated. A 2-mm hole was made at the corneal limbus, the eyes were fixed with $4 \%$ paraformaldehyde for $10 \mathrm{~min}$, the anterior chamber was dissected, and the lens was removed. Eyecups for cryosections were then fixed for 50 min in $4 \%$ paraformaldehyde in PBS solution before cryoprotection in $20 \%$ sucrose overnight at $4{ }^{\circ} \mathrm{C}$. Eyecups were embedded in Tissue-Tek optimal cutting temperature compound (Sakura) and fastfrozen in a dry ice-ethanol bath. Blocks were sectioned with a Leica CM1850 Cryostat, and 18 $\mu \mathrm{m}$ sections were mounted on Superfrost Plus slides. Cryosections were washed in PBS solution and incubated in blocking buffer [2\% goat serum (Invitrogen), $0.1 \%$ Triton $\mathrm{X}-100$, and $0.05 \%$ sodium azide in PBS solution] for $1 \mathrm{~h}$ and then with primary antibodies for $4 \mathrm{~h}$ at room temperature or overnight at $4^{\circ} \mathrm{C}$. The slides were washed three times for 10 -min each in PBS solution with $0.1 \%$ Triton $\mathrm{X}-100$ to removed excess antibody and incubated with secondary antibody for $45 \mathrm{~min}$ at room temperature. Slides were washed twice for 10 min with PBS solution with $0.1 \%$ Triton X-100 and for $10 \mathrm{~min}$ in PBS solution. ProLong Gold antifade reagent (Invitrogen) was applied to each section, and coverslips were mounted. Images were collected with a Zeiss LSM 510 Meta confocal microscope using 488-, 543-, and 633-nm laser lines. The 
following antibodies were used: anti-CNGA1/3 (University of California, Davis/ National Institutes of Health NeuroMab Facility), anti-PDE $\beta$ (Affinity Bio Reagents), anti-G $\gamma \mathrm{T} 1$ (Santa Cruz Biotechnology), anti-G $\gamma$ T2 (gift from Dr. Vadim Arshavsky, Duke University Eye Center, Durham, NC), anti-PDE6 $\alpha^{\prime}$ (3184p) $(84,86,120)$ and anti-GRK1 (Ching-Kang Chen, Virginia Commonwealth University, Richmond, VA). DAPI nuclear stain (Invitrogen) was added to dilutions of Alexa Fluor secondary antibodies (Invitrogen) in antibody dilution buffer (0.05\% goat serum, $0.1 \%$ Triton $\mathrm{X}-100$, and $0.05 \%$ sodium azide in $1 \times$ PBS solution.

Statistics. Data plotted as mean and standard error of mean. Immunoblotting, distribution of cone photoreceptor cells and ERG data were analyzed by students-t test. 


\section{SUPPLEMENTAL FIGURES}

Fig. S1. Generation of mice with cone-specific conditional removal of prenyl transferases. ( $A$ and $B$ ) Scheme showing the cone photoreceptor-specific knockouts of Pggtlb (A) and Fntb (B). $(C$ and $E$ ) Cre expression in mouse cone photoreceptors lacking Pggtlb $(C)$ or Fntb (E). Peanut agglutinin (PNA) labels the cone sheath. Cre-specific antibody staining (green) is detected in cones in retinal cross sections from Pggtlb ${ }^{--}$mice (C, top, dashed box $)$and $\mathrm{Fntb}^{--}$(E, top, dashed box). In comparison, $\mathrm{Cre}$ is not expressed in littermate controls. Nuclei are stained with DAPI (blue). (D) Immunoblots probed with $C r e$-specific antibody show Cre expression in Crepositive mouse lines $\left(\mathrm{Pggtl}^{---}\right.$and $\left.\mathrm{Fntb}^{--}\right)$. GAPDH was used as a loading control. OS, outer segment; IS, inner segment; ONL, outer nuclear layer.

Fig. S2. Progressive loss of photoreceptor function in mice lacking protein geranylgeranylation. $(A$ and $B$ ) Graphs showing the amplitudes of "b" waves versus age in Pggtlb and Fntb littermates. $n=4)$.

Fig. S3. Rod photoreceptor function is unaltered in mice with cone-specific deletion of prenyl transferases. Representative waveforms of rod ERG responses recorded from $\mathrm{Pggtlb}^{-/}$mice and $\mathrm{Pggtl}^{+/+}$littermate controls at P85 (A) and $\mathrm{Fntb}^{-/-}$mice and $\mathrm{Fntb}^{+/+}$littermate controls at P180 (B). Representative rod waveforms, measured at $-0.8 \log \mathrm{cd} * \mathrm{~s} / \mathrm{m}^{-2}$ (top) and $0.4 \log \mathrm{cd} * \mathrm{~s} / \mathrm{m}^{-2}$ (bottom), $n=3$. ( $n$, number of littermates of each genotype).

Fig. S4. Defective protein farnesylation does not affect the protein levels in cone photoreceptors. (A) Representative immunoblots of retinal protein extracts from P180 Fntb $\mathrm{Fice}^{-/}$mice and $\mathrm{Fntb} \mathrm{b}^{+/+}$ littermate controls probed with antibodies against the indicated proteins. (B) Quantitation of results derived from panel A. Integrated intensity values were normalized to $\alpha$-tubulin. $n=4$. 
Fig. S5. Membrane association of cone-specific proteins does not require protein farnesylation. Isotonic cellular fractionation of P30 retinal lysates of $\mathrm{Fntb}^{-/-}$mice and $\mathrm{Fntb}^{+/+}$littermate controls, followed by western blotting. Retinal RetGC1 and AIPL1 served as controls. $n=3$ of each $\mathrm{Fntb}^{-/-}$mice and $\mathrm{Fntb}^{+/+}$littermate.

Fig. S6. Localization of GRK1 and cone PDE6 is unaffected in the absence of protein farnesylation. Immunofluorescence labeling of retinal cross-sections in $\mathrm{Fntb}^{-/-}$mice and $\mathrm{Fntb} \mathrm{b}^{+/+}$ littermate controls. Nuclei are stained with DAPI (blue). (A) GRK1 (red) appears in both cone and rod photoreceptors; cone PDE6 (green) co-localizes with GRK1 in cones (top). (B) PNA (red) labels the cone sheath, and M-opsin (green) co-localizes with PNA (top). Scale bar, $10 \mu \mathrm{M}$.

Fig. S7. Cone transducin localization is unaffected in the absence of protein geranylgeranylation. Immunofluorescence labeling of retinal cross-sections in $\mathrm{Pggtl}^{-/}$mice and $\mathrm{Pggtl}^{+/+}$littermate controls at P85. (A) GaT2 (green) co-localizes with peanut agglutinin (PNA), a cone marker. (B) GyT2 (green) co-localizes with PNA (red). Nuclei are stained with DAPI (blue). Scale bar, 10 $\mu \mathrm{M}$. 
FIGURES

A

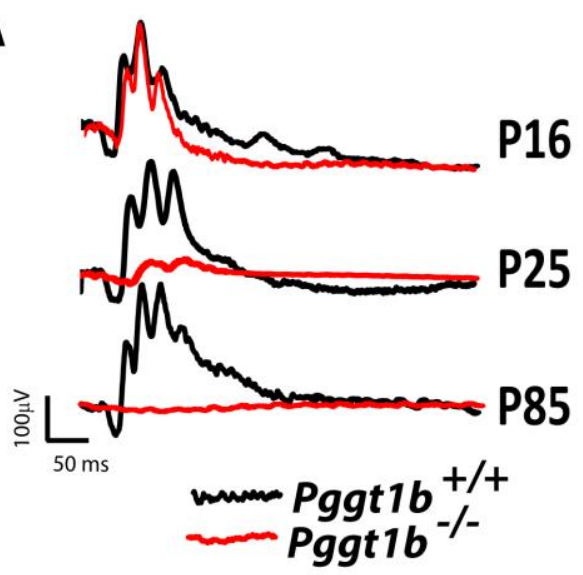

C
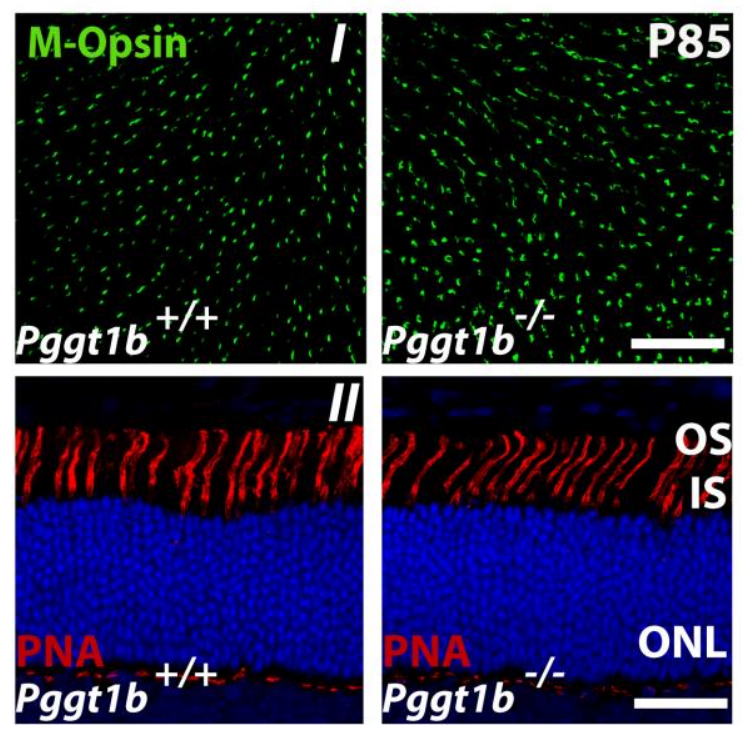

B

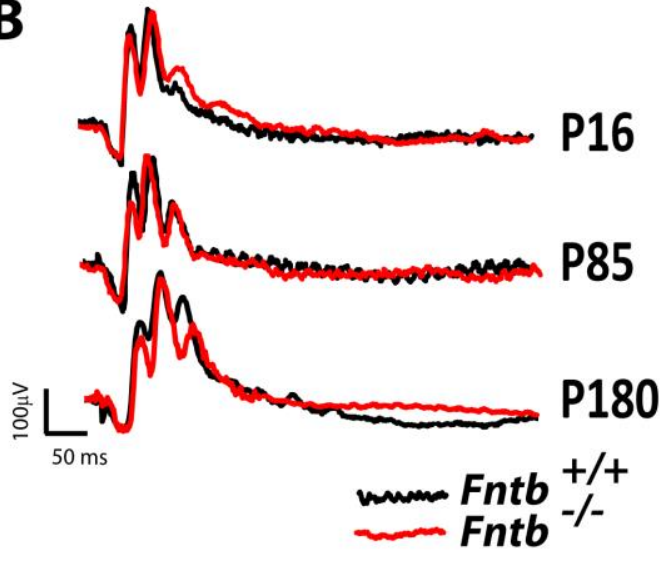

D
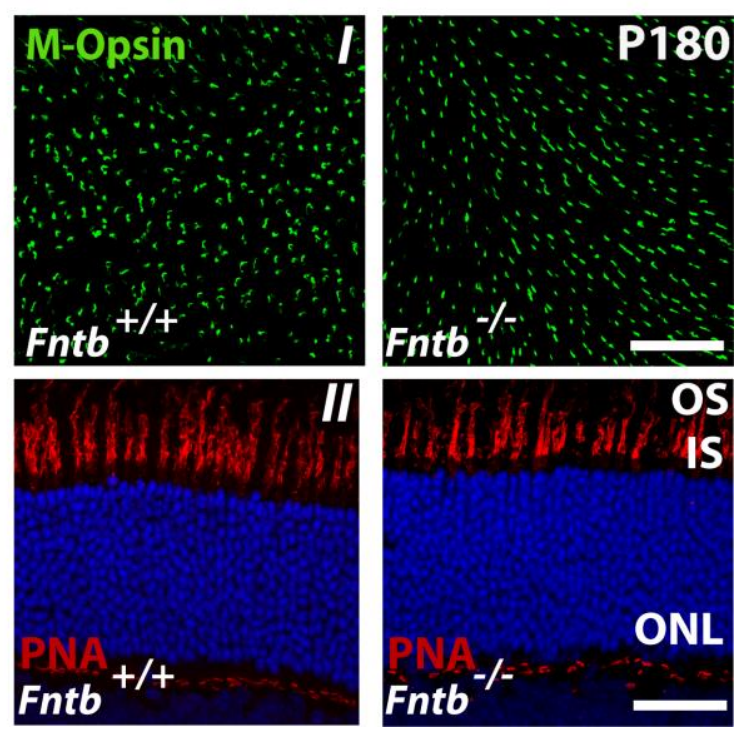

Fig. 1 Protein geranylgeranylation is crucial for cone photoreceptor function. 


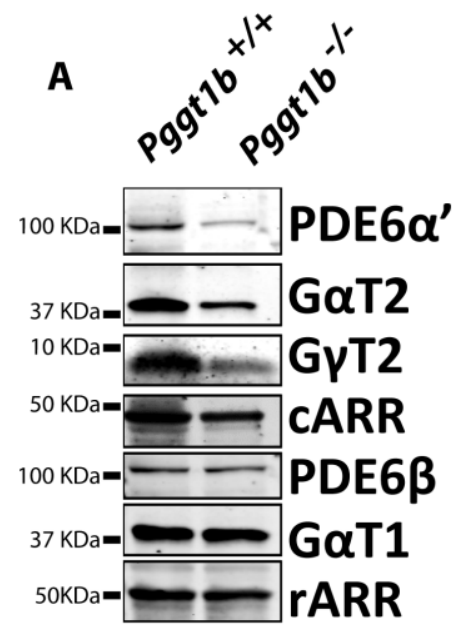

B $\quad$ Pggt $1 b^{+/+} \square{\text { Pggt } 1 b^{-/-}}^{-}$

P85

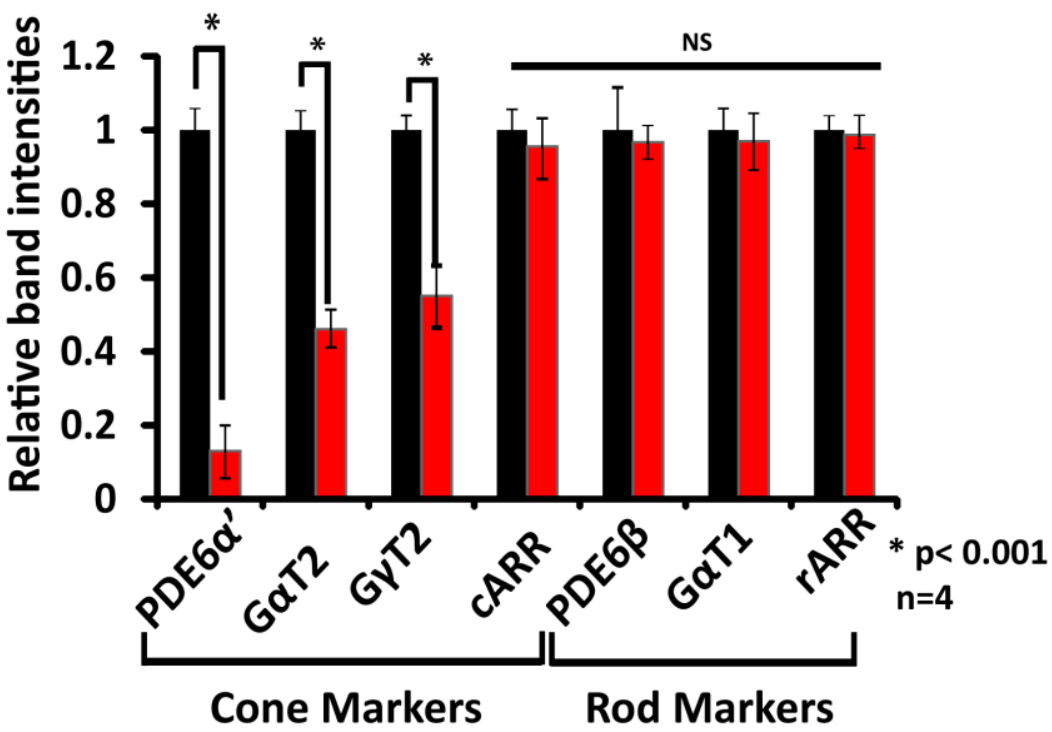

Fig. 2 Defective protein geranylgeranylation leads to reduction in the levels of phosphodiesterase and transducin in cone photoreceptors. 
A

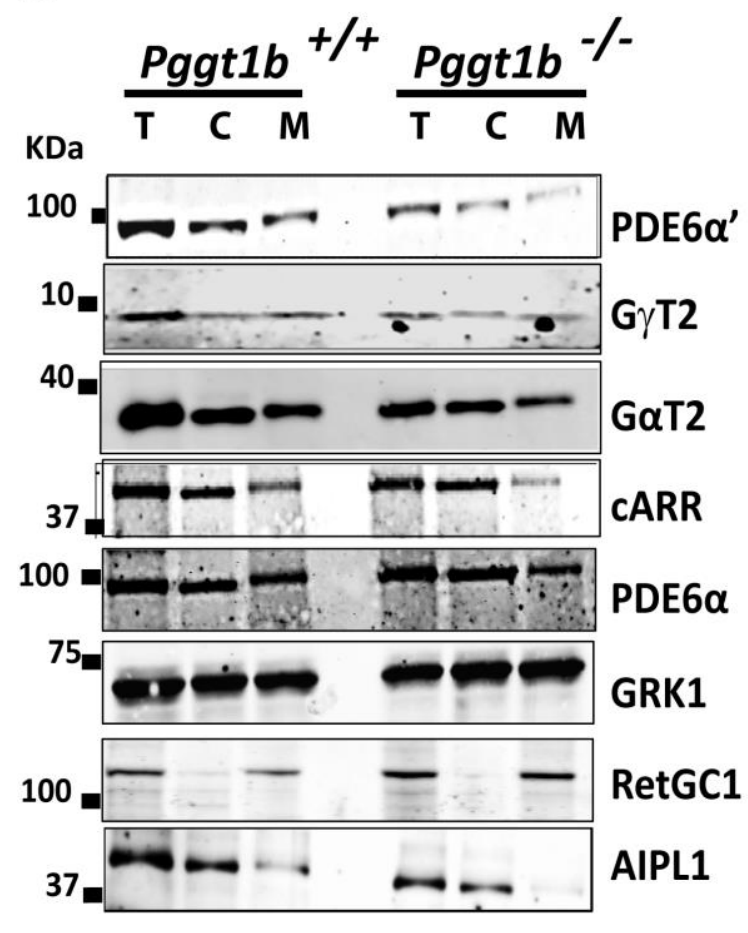

B

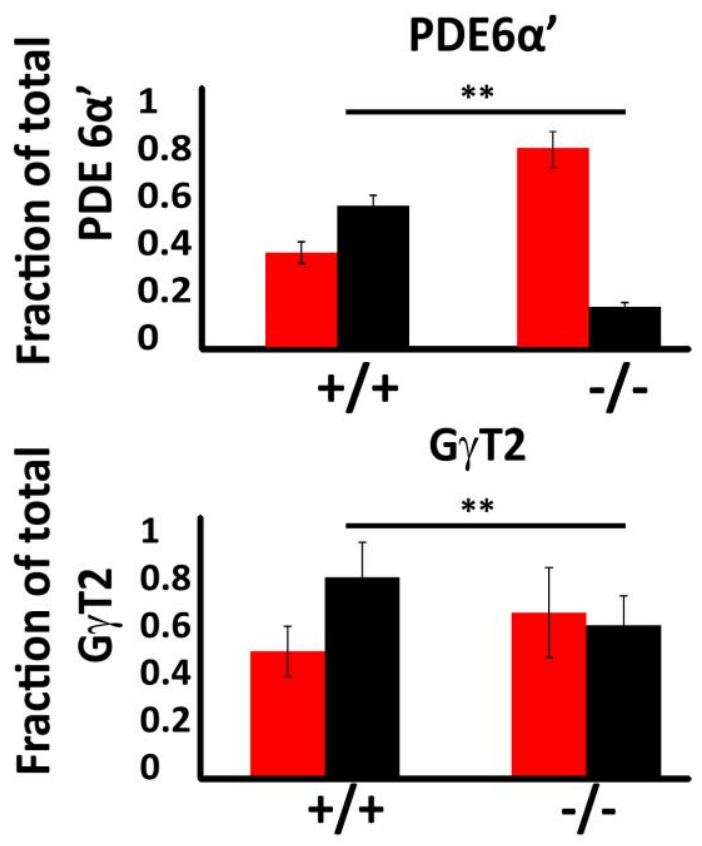

Fig. 3 Impaired membrane partitioning of cone phosphodiesterase and transducin in the absence of Pggtlb. 
A

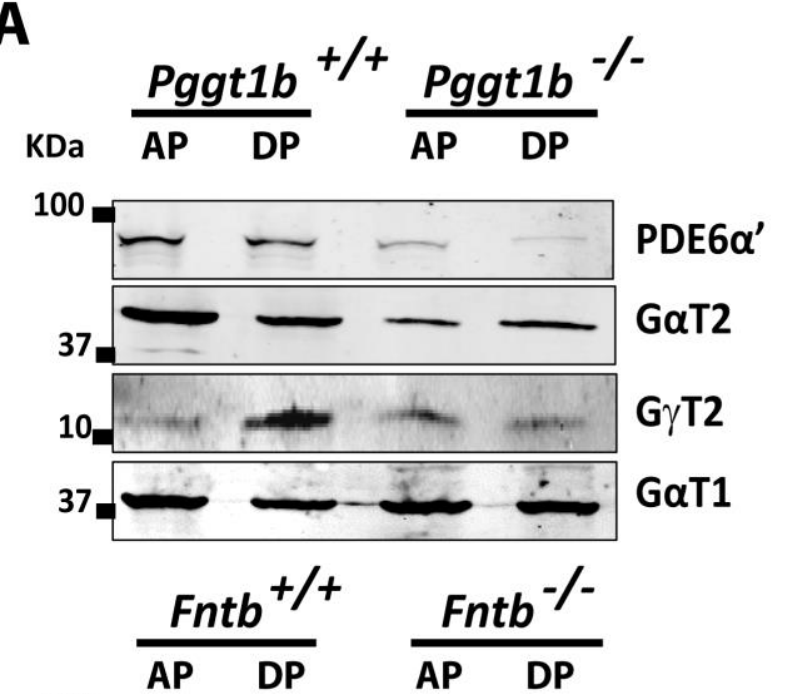

B
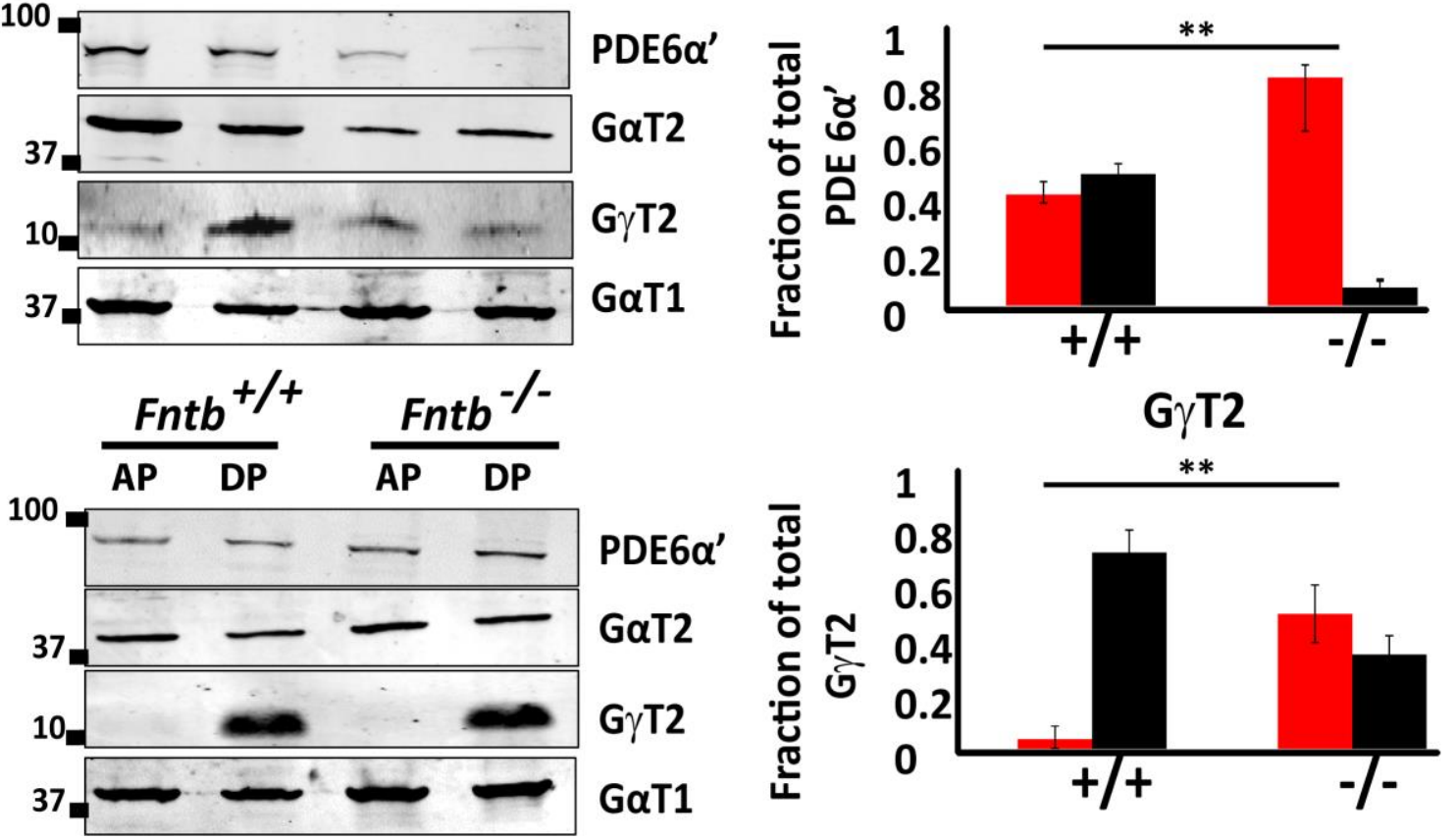

Fig. 4 Inefficient partitioning of cone phosphodiesterase and cone transducin- $\gamma$ subunit in Triton X-114 detergent in the absence of Pggt1b. 


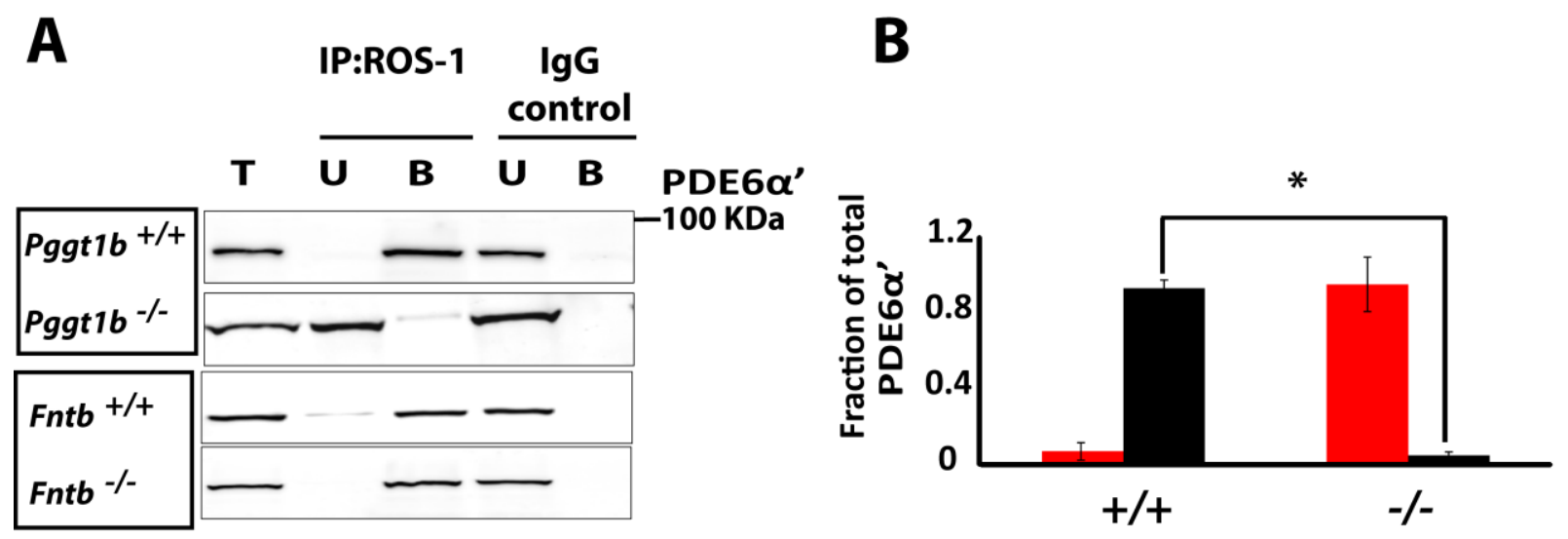

Fig. 5 Pggtlb-mediated protein geranylgeranylation is important for the assembly of cone phosphodiesterase. 
A

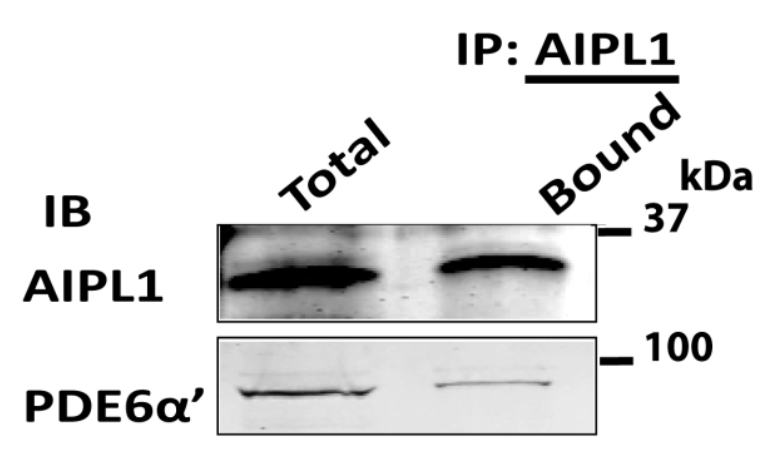

B

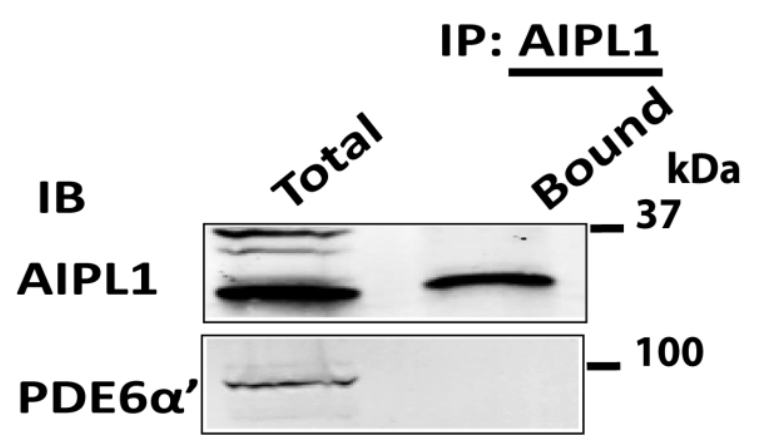

Fig. 6 Interaction of AIPL1 with cone phosphodiesterase requires protein geranylgeranylation. 
A
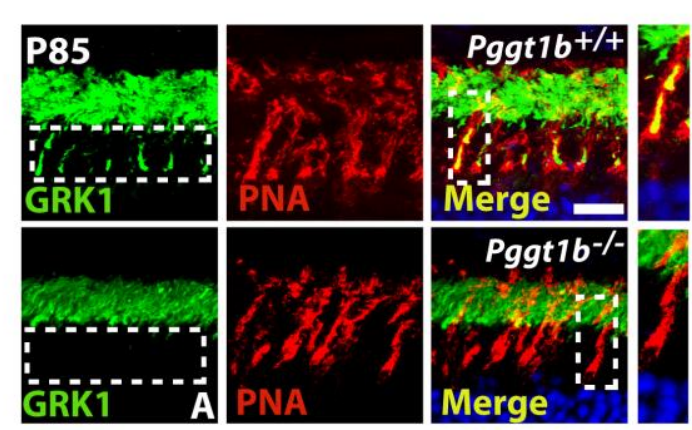

C
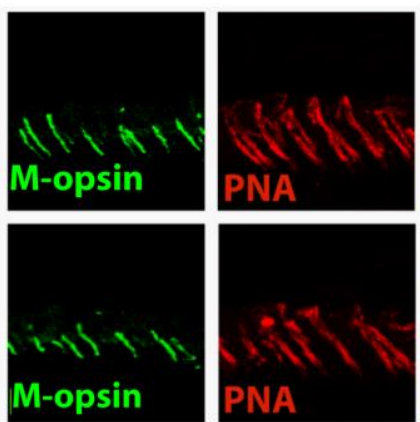

B
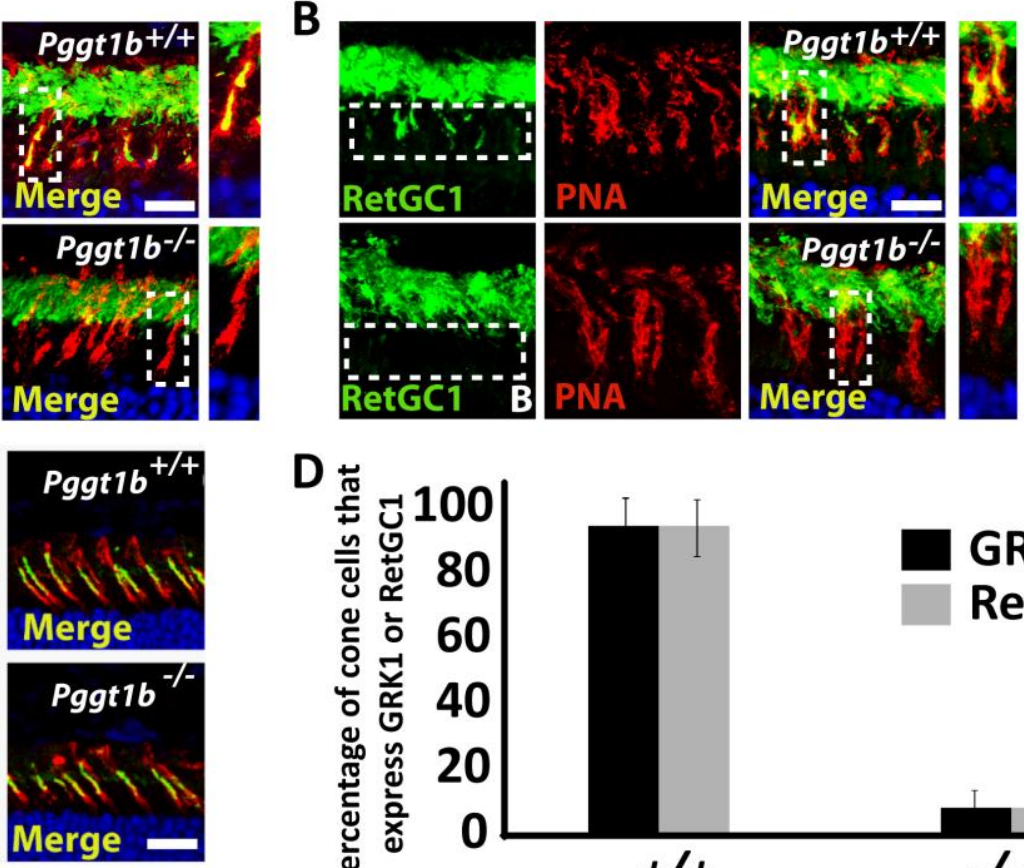

D

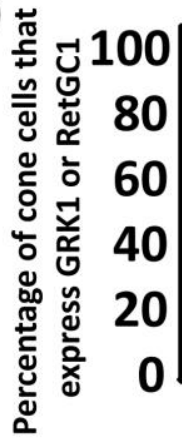

GRK1 RetGC1

Fig. 7 Rhodopsin kinase (GRK1) and Retinal guanylate cyclase (RetGC1) are absent in cones lacking Pggt1b. 

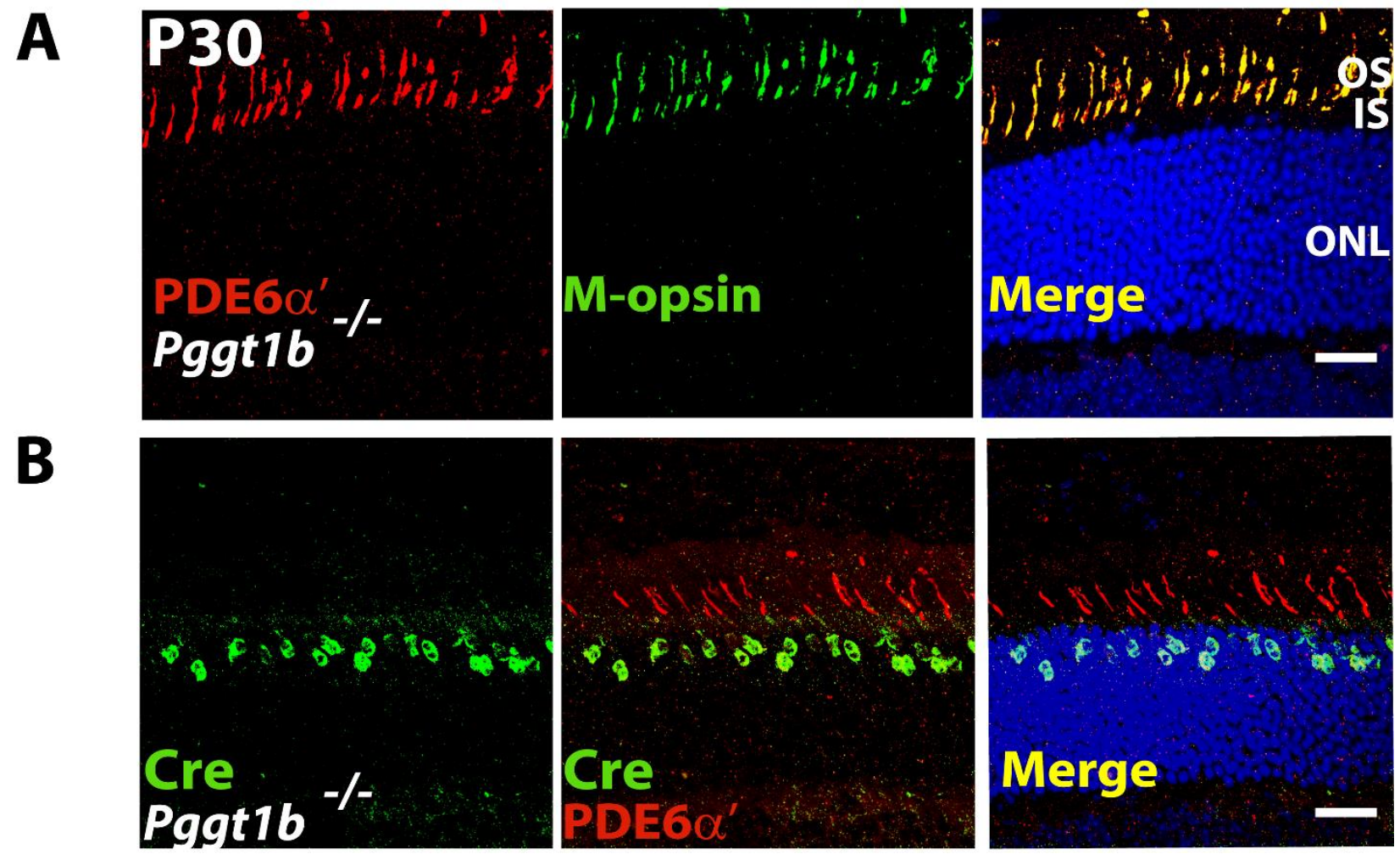

Fig. 8 Normal trafficking of phosphodiesterase to the cone OS in the absence of protein geranylgeranylation. 


\section{Cone-specific Pggt1b Conditional Knockout}
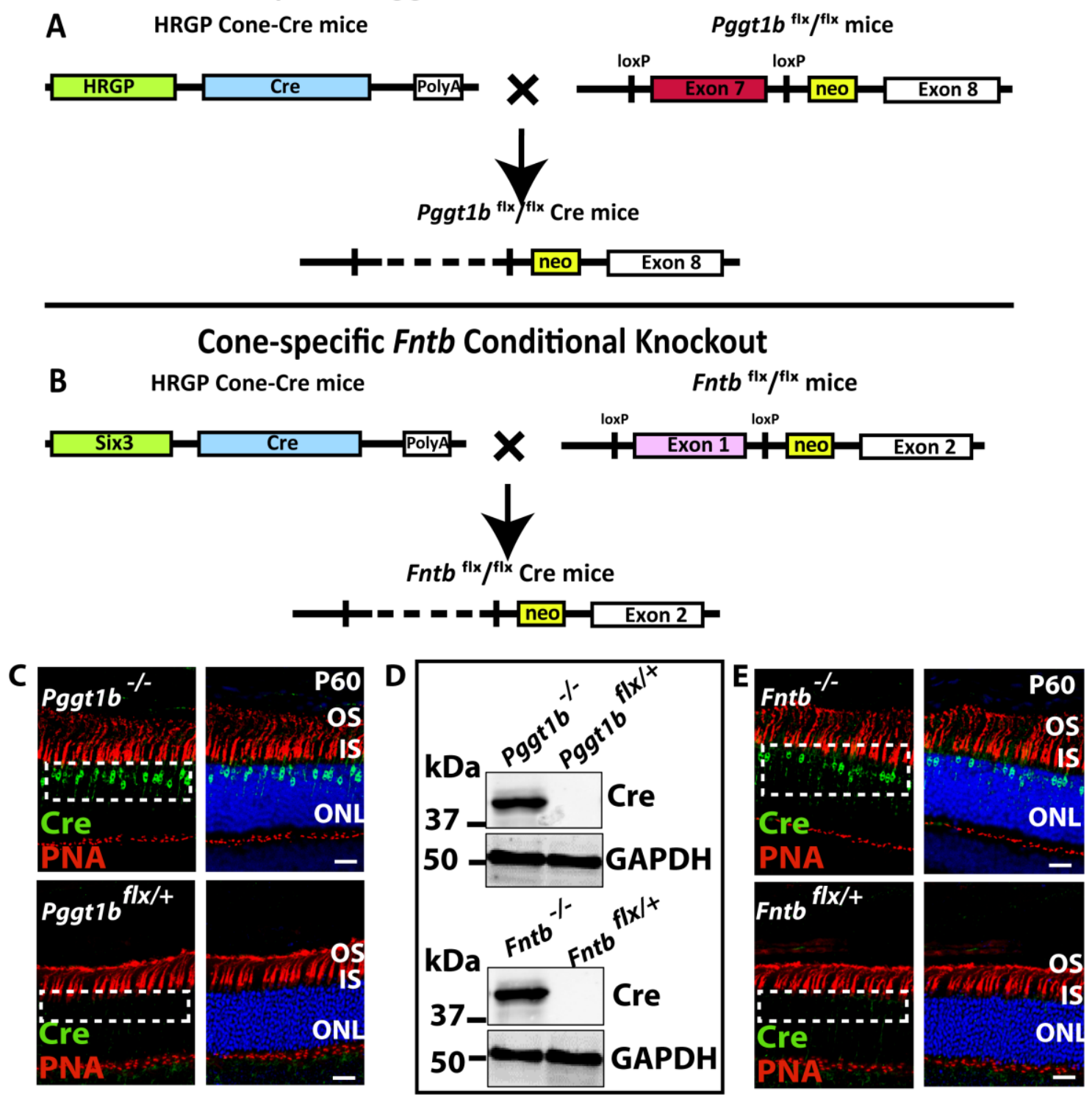

Fig. S 1 Generation of mice with cone-specific conditional removal of prenyl transferases. 

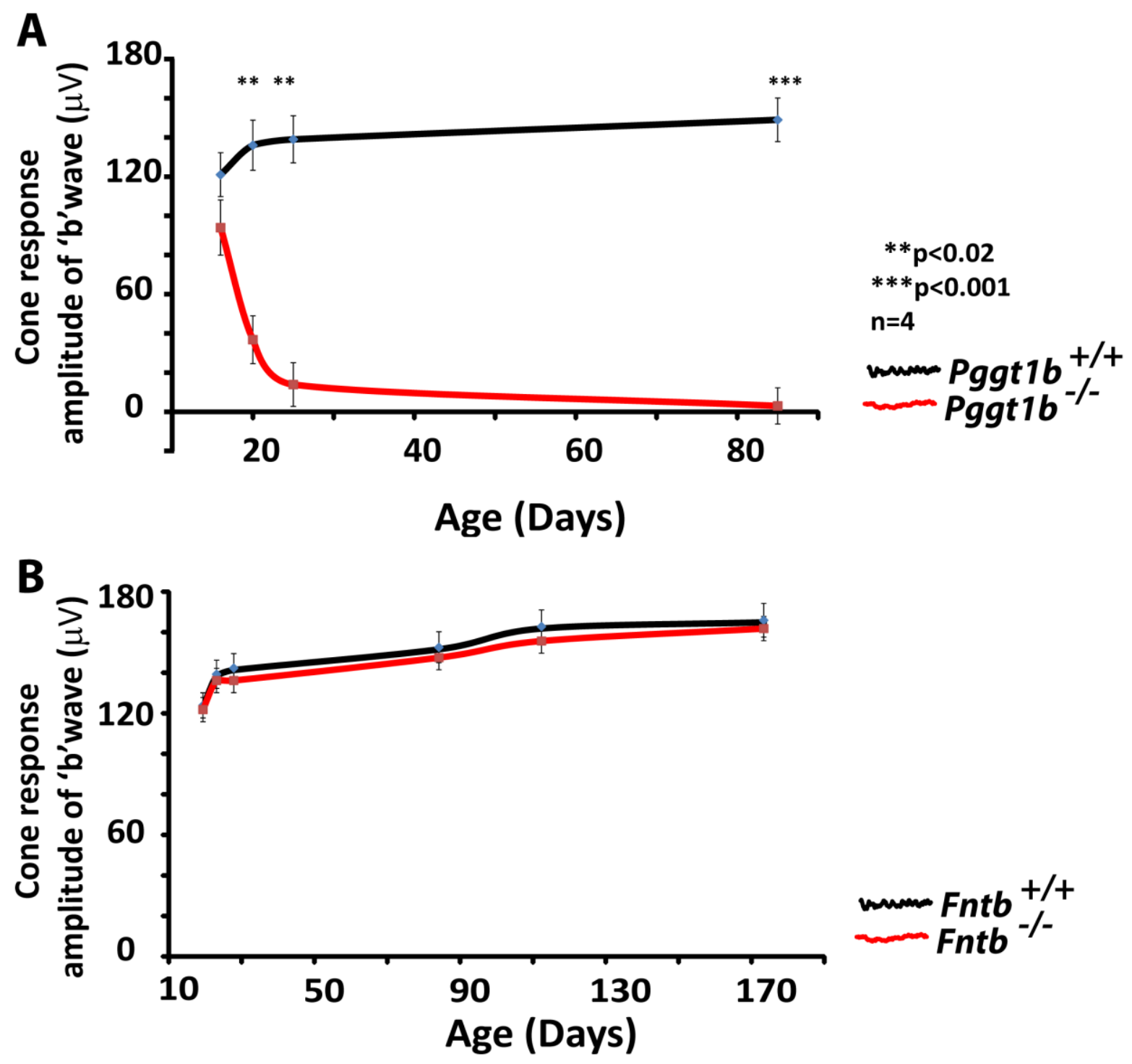

Fig. S 2 Progressive loss of photoreceptor function in mice lacking protein geranylgeranylation. 

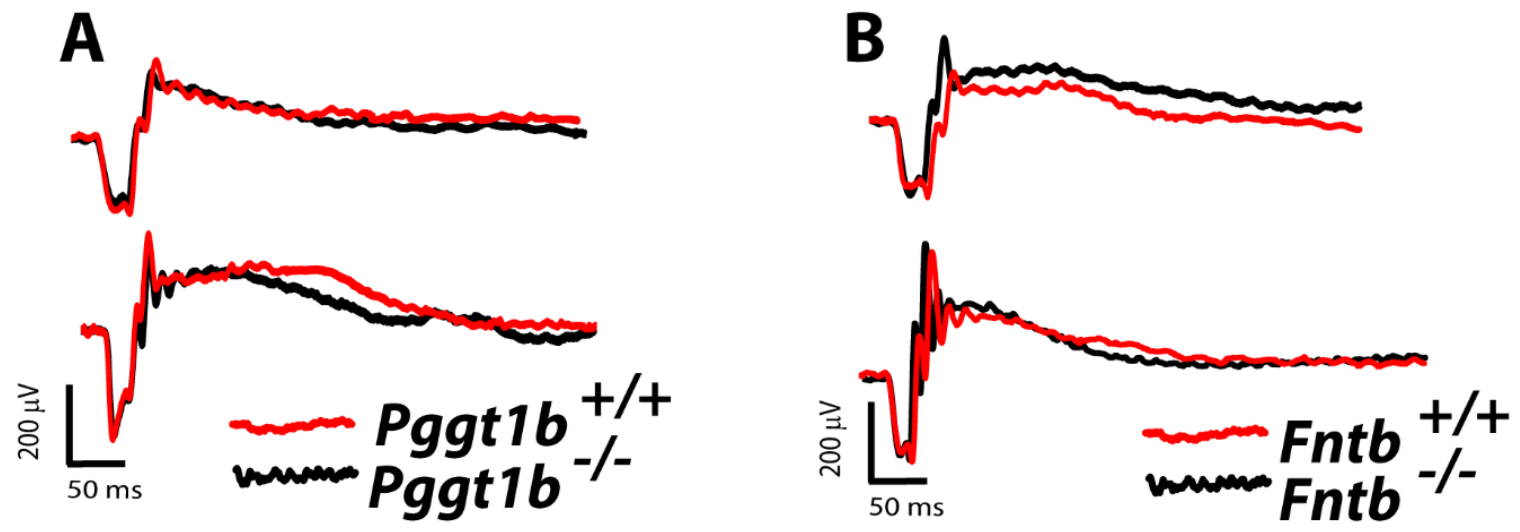

Fig. $\mathbf{S} 3$ Rod photoreceptor function is unaltered in mice with cone-specific deletion of prenyl transferases. 


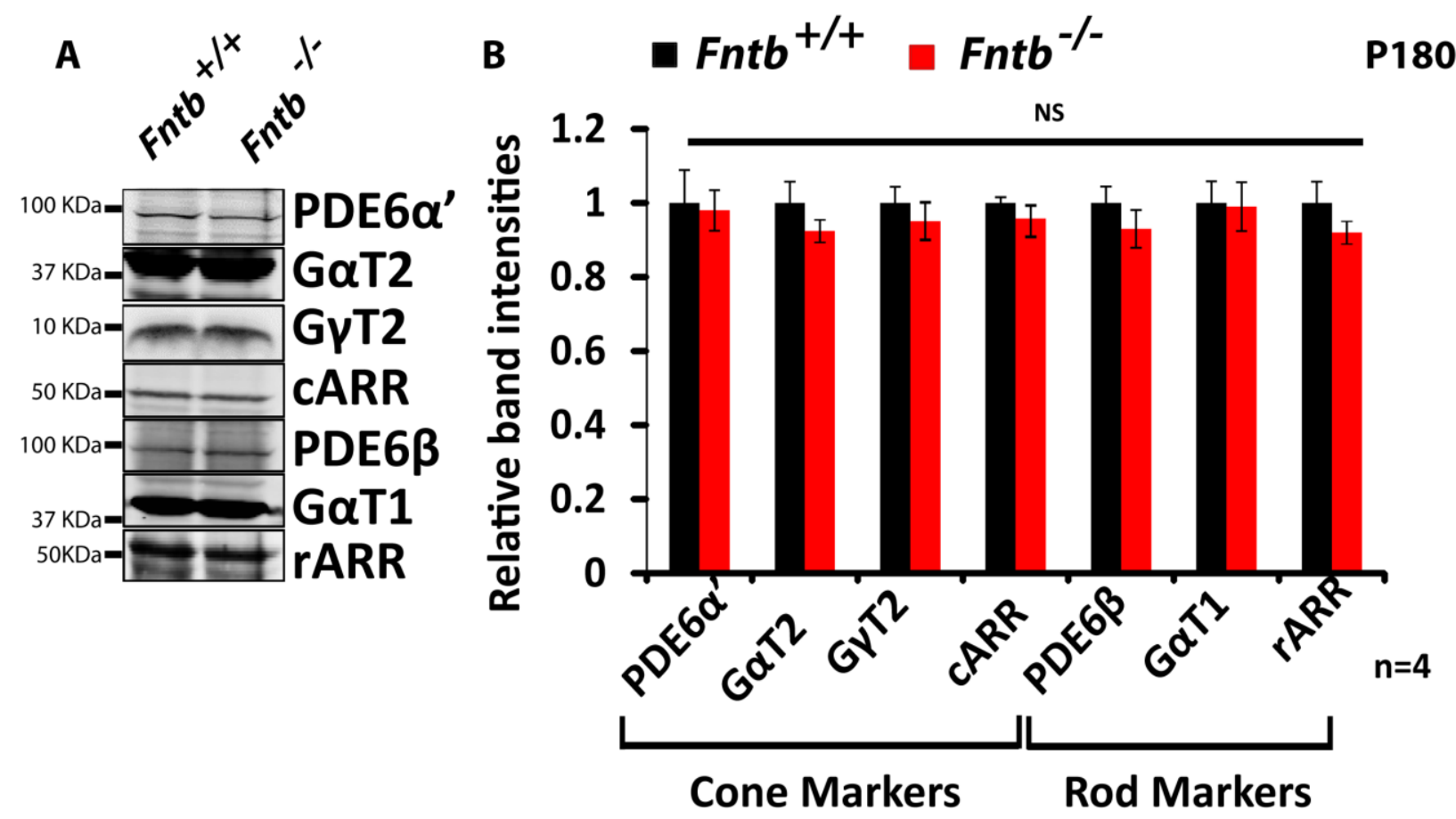

Fig. S 4 Defective protein farnesylation does not affect the protein levels in cone photoreceptors. 


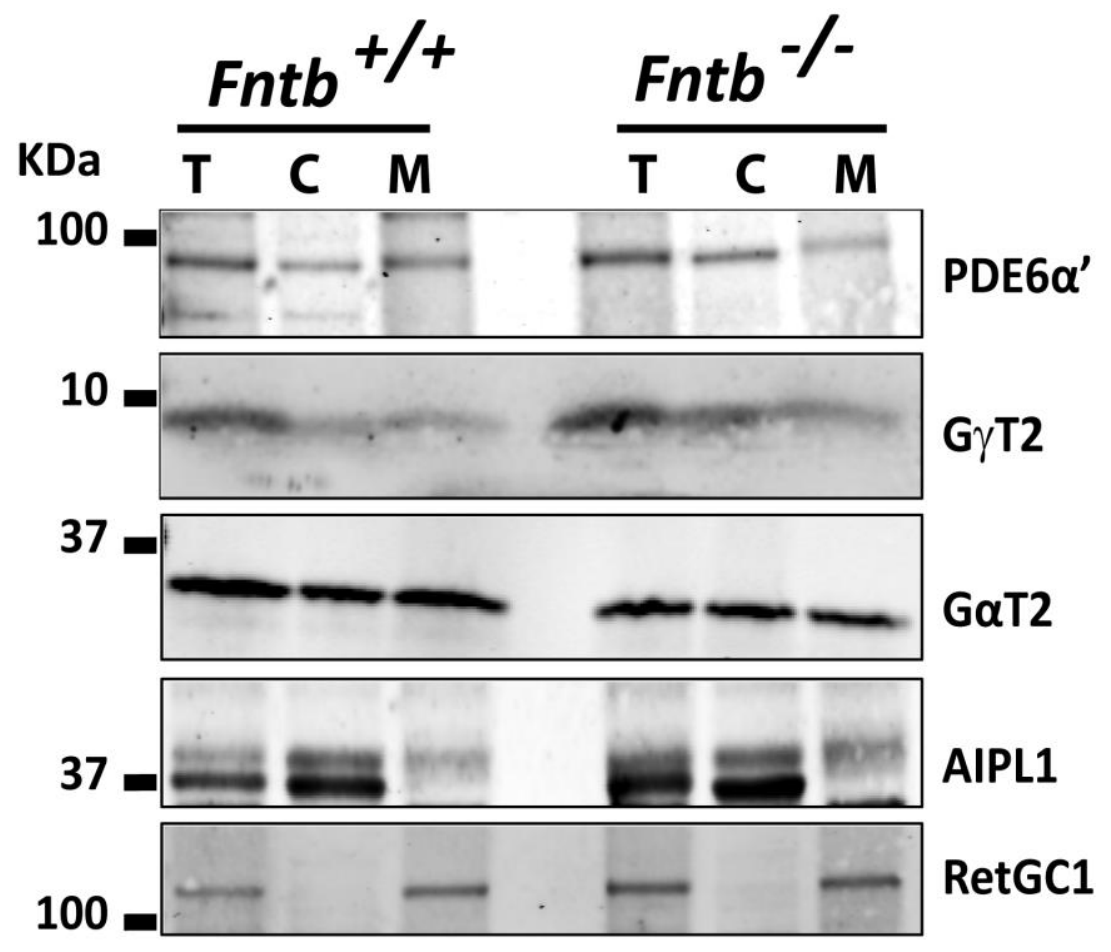

Fig. S 5 Membrane association of cone-specific proteins does not require protein farnesylation. 

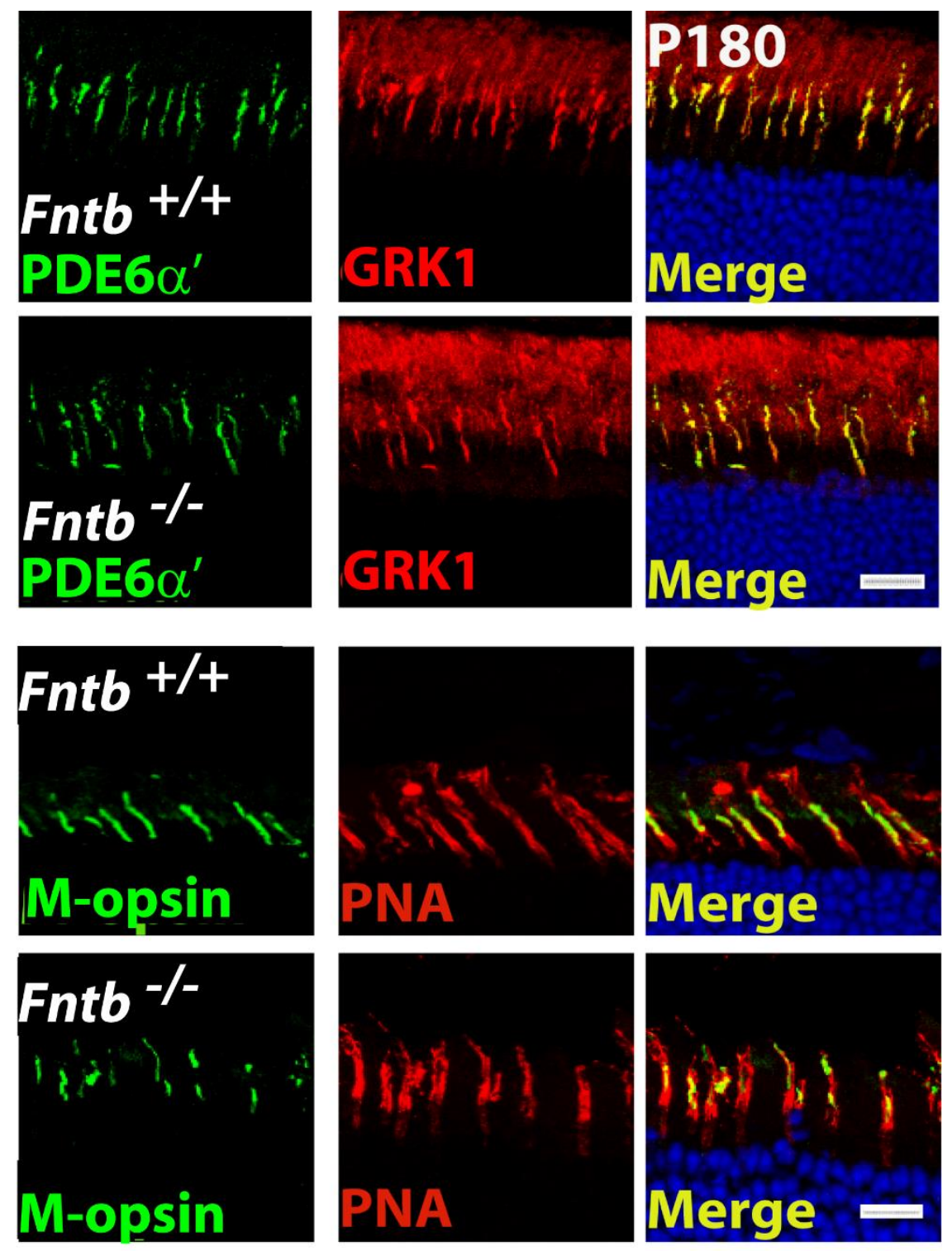

Fig. S 6 Localization of GRK1 and cone PDE6 is unaffected in the absence of protein farnesylation. 

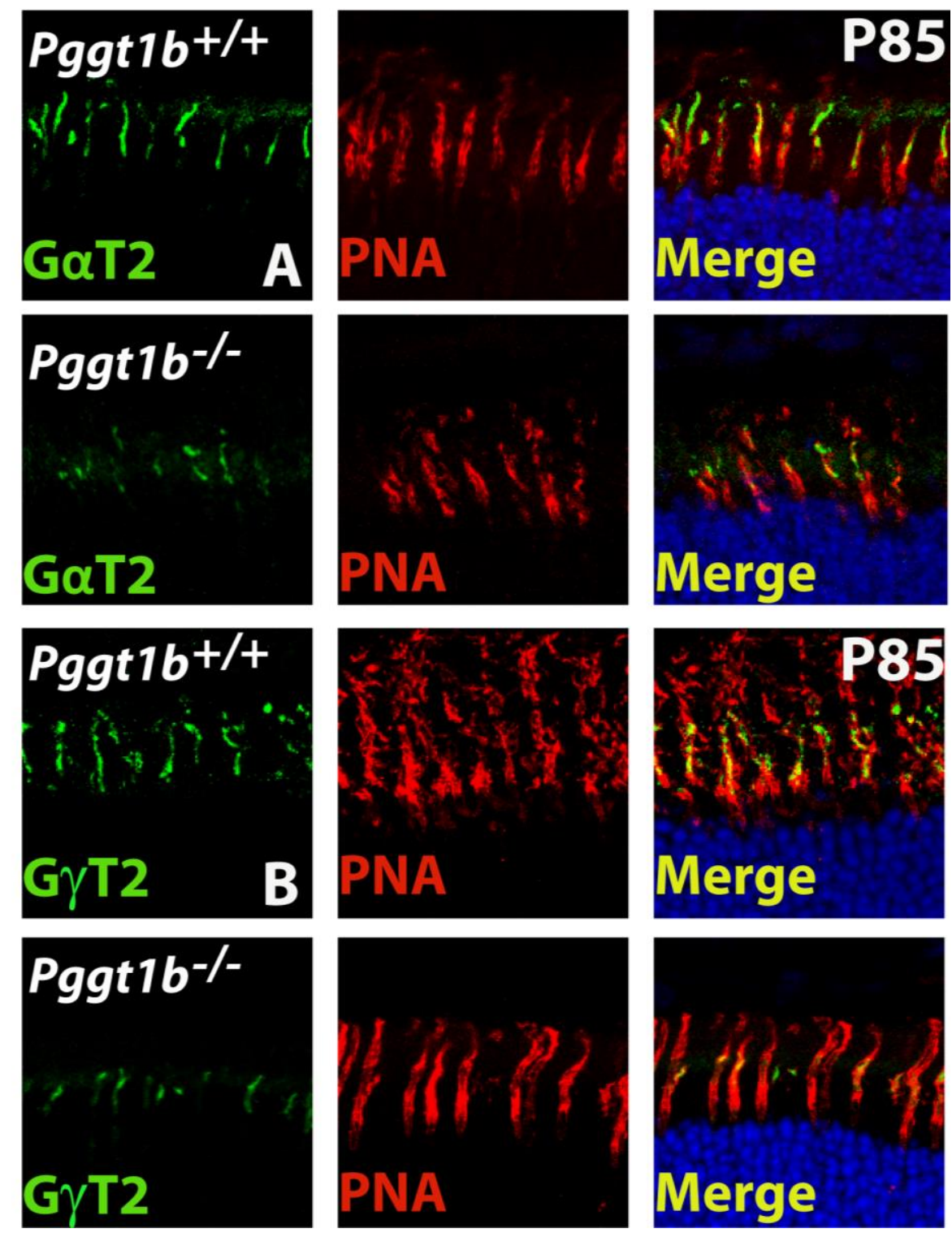

Fig. $\mathbf{S} 7$ Cone transducin localization is unaffected in the absence of protein geranylgeranylation. 


\section{CHAPTER 3}

\section{Genetic ablation of prenyl transferases leads to loss of photoreceptor function}

\section{Nachiket D. Pendse ${ }^{\mathrm{a}}$, Martin O. Bergo ${ }^{\mathrm{b}}$, Stephen G. Young ${ }^{\mathrm{c}}$, and Visvanathan}

Ramamurthy $\mathbf{y}^{\mathrm{a}, 1}$

a'Departments of Ophthalmology, Biochemistry and Biology, West Virginia University,

Morgantown, WV; bSahlgrenska Cancer Center, Gothenburg, Sweden; and 'Departments of

Medicine and Human Genetics, University of California, Los Angeles, CA

To whom correspondence should be addressed. E-mail: ramamurthyv@wvumedicine.org.

\section{Author Contributions}

N.D.P. and V.R. designed the research and wrote the manuscript; N.D.P. performed the research;

N.D.P. and V.R. analyzed the data; M.O.B. and S.Y. provided advice and reagents and helped write the manuscript. We thank Thamarai Saravanan for maintaining animal stocks and Dr.

Karen Martin for advice and the use of the West Virginia University Microscopic Imaging Facility. We also thank members of the Ramamurthy laboratory for constructive criticism. This work was supported by National Institutes of Health Grants R01EY017035 (to V.R.) and R01HL126551 (to S.Y.), West Virginia Lions, and an Unrestricted Research to Prevent Blindness Challenge Grant (West Virginia University).

Key words: Prenylation, photoreceptor neurons, phosphodiesterase, protein assembly, protein trafficking, transducin translocation

Manuscript information: Abstract: 248, Significance statement: 117, Introduction 615, Materials and methods 970, results 3009, Discussion 1160, Total 50 pages and 10 figures. 


\begin{abstract}
Variety of phototransduction proteins in photoreceptor neurons are post-translationally lipid modified at their C-terminal " $-C A A X$ ” motif by a process known as prenylation. These include transducin- $\gamma(\mathrm{G} \gamma \mathrm{T} 2)$, G-protein coupled rhodopsin kinase (GRK1) and catalytic subunits of phosphodiesterase (PDE6). Prenylation is thought to be needed, not only for anchoring membranes of proteins but also in trafficking and regulating interactions between proteins. However, in-vivo evidence examining the role of prenylation in retinal neurons is absent. In the present investigation, we generated mice lacking Pggtlb (catalytic subunit of geranylgeranyl transferases) or Fntb (catalytic subunit of farnesyltransferases) using Six3 promoter that drives the expression of Cre recombinase in retinal neurons. While photoreceptor development progressed normally at early stage (post-natal day, P16), in the absence of prenylation, the photoreceptor function was abolished progressively which was accompanied by shortening of photoreceptor outer segments (OS) and severe photoreceptor degeneration in mice deficient in $P g g t 1 b$ or Fntb. Lack of prenylation resulted in defective association of prenylated rod transducin and PDE6 with photoreceptor disk membranes which further resulted in their decreased protein levels. The rod transducin was mislocalized and its translocation kinetics were severely affected in the absence of Fntb as shown by slow re-appearance of translocated transducin to OS. Rod PDE6 was assembled properly and was transported to the OS in the Pggtlb or Fntb deficient mice. In addition to these defects, we also observed reduced levels of G-protein-coupled receptor kinase (GRK1) in Pggt1b or Fntb deficient mice. Overall our findings demonstrate that prenylation is crucial for normal trafficking of G-protein transducin, function and survival of photoreceptors.
\end{abstract}




\section{SIGNIFICANCE STATEMENT}

Protein prenylation is thought to increases the hydrophobicity of proteins that is needed for membrane anchorage of proteins. Fascinatingly, although multiple prenylated proteins are found in neurons, nothing is known about the importance of prenylation in regulating protein-protein interaction, assembly and their trafficking in retinal neurons. Here we show that, prenylation is crucial not only for survival and function of photoreceptor neurons but also needed for proper trafficking of G-protein. Multiple disease such as rod and cone dystrophy, retinitis pigmentosa, Leber's congenital amaurosis have been linked to the defects in PDE6, Rab proteins and transducin. Our studies are first to tie defects in prenylation of PDE6 and transducin with the visual dysfunctions. 


\section{INTRODUCTION}

Protein farnesyltransferase (FTase) and protein geranylgeranyl transferase-I (GGTase-I) adds 15-carbon farnesyl or 20-carbon geranylgeranyl lipids respectively, to the cysteine in proteins that terminate with a " $-C A A X$ " motif (" $C$ " is a cysteine; " $A$ " is often an aliphatic amino acid; the " $X$ " can be one of many residues) $(15,19,31)$. This post-translational modification is generally called "protein prenylation". Cell culture studies have demonstrated that protein prenylation promotes the ability of proteins to associate with membrane surfaces. For example, when the farnesylation of H-RAS (a proto-oncogene associated in human cancers) is eliminated, the protein loses its ability to anchor to the plasma membrane and relay the signals that promote cell growth (129-131). The studies with the germline knockouts of Fntb (encoding the catalytic subunit of FTase) or Pggtlb (encoding the catalytic subunit of GGTase-I) are embryonic lethal indicating the importance of protein farnesylation and geranylgeranylation in normal development (33). Tissue specific removal of Fntb or Pggtlb shows the need for protein prenylation in normal functioning of liver, kidney, skin, brain and heart (20,132-135,136, Lee, 2010 \#104, Khan, 2011 \#105). Altogether, these results emphasize the crucial role played by protein prenylation in various tissue types.

Many years of research have identified key players in phototransduction residing in photoreceptor outer segments (POS). Interestingly, many of these proteins are prenylated, a modification thought to be needed for anchoring the membranes in numerous disks present in the POS. These include transducin- $\gamma$, G-protein coupled rhodopsin kinase (GRK1) and phosphodiesterase (PDE6) $(36,69,108-110,116)$. However, experimental proof investigating the role of prenylation is absent. 
Rod PDE6 is the crucial effector enzyme in phototransduction pathway $(66,116,137)$. Rod PDE6 is composed of two catalytic subunits, a $\alpha$ subunit which is farnesylated and a $\beta$ subunit which is geranylgeranylated $(67,109,113,116,138)$ and two inhibitory subunits $(\gamma)(63)$. Studies on the rod PDE6 have suggested that prenylation of PDE6 is needed for proper anchoring of rod PDE6 to the OS disk membranes $(119,137)$. Alternatively, we previously proposed that the prenylation at the c-terminus of PDE6 is needed for chaperone, aryl hydrocarbon receptor interacting protein like-1 (AIPL1) to fold and assemble functional PDE6 in the photoreceptor inner segments.(84-86). However, the in-vivo requirement for lipid modification in rod PDE6 assembly and function is not clear.

Rod transducin is a heterotrimeric $\mathrm{G}$ protein that consists of a myristoylated $\mathrm{G} \alpha \mathrm{T} 1$ subunit, a non lipidated G $\beta 1$ subunit, and a G $\gamma \mathrm{T} 1$ subunit which is farnesylated $(36,139-141)$. We recently demonstrated that ICMT mediated methyl esterification of rod transducin- $\gamma(\mathrm{G} \gamma \mathrm{T} 2)$ is essential for its stability and plasma membrane interaction (59). An interesting property of transducin complex is the light dependent translocation from rod outer segment (OS) to inner retina, which is thought to be a neuroprotective mechanism $(13,68,142,143)$. The lipid anchors are thought to regulate this process (74) but in effect how it impacts the return of translocated transducin subunits to rod outer segment and what controls this dynamic process is unclear. Here we illustrate the unique requirement of farnesylation for proper return of translocated transducin in rod photoreceptors.

The role of prenylation has been demonstrated in maintaining architecture of retinal neurons using intravitreal injections of HMG-Co-A inhibitors. Their use resulted in disruption of retinal architecture and lamination suggesting the importance of prenylation in retinal neurons. 
However major drawback of these studies is that statins also affect endothelial cells in blood vessels resulting into cellular dysfunction and cell death (144).

To examine the role of prenylation in photoreceptors neurons, we made conditional animal model by breeding $F n t b^{f l / f l}$ or $P g g t l b^{f l / f l}$ mice with mice expressing Cre recombinase under the control of the retina and forebrain-specific promoter Six3 (145). This animal model was used to identify cellular, molecular and functional consequences of lack of prenylation in retinal neurons. 


\section{MATERIAL AND METHODS}

\section{Mouse models}

Mice homozygous for $F n t b$ conditional knockout alleles $\left(F n t b^{f l / f l}\right)$ were bred with mice hemizygous for Six3-Cre transgene to create $F n t b^{f l / f l}$ Six3-Cre mice, which have a retina-specific inactivation of Fntb (145). Littermate $F n t b^{w t / f l}$ Six3-Cre mice were used as controls. Similar strategy was used to generate Pggtl ${ }^{f l / f l}$ Six3-Cre by crossing Pggt $1 b^{f l / f l}$ mice with littermate $P g g t 1 b^{w t / f l}$ Six3-Cre. Genotyping was performed by PCR amplification using genomic DNA extracted from mouse ear punch biopsies sample, as described earlier $(34,146)$. Both males and females were used in the study. Mouse experiments were performed in accordance with the National Institute of Health guidelines and the protocol approved by Institutional Animal Care and Use Committee of West Virginia University.

\section{Quantitative-PCR}

Retinas were dissected from freshly enucleated eyes and frozen on dry ice in the presence of Trizol (Invitrogen). RNA extracted from frozen retinas was used to generate cDNA as described by (59). Fntb was amplified as described by (146) with primers, localized within the floxed segment (exon1- 6), were 5'-CAATTAGGCGAGAGCG AAAC-3'(exon1) and 5'GCAGGAGATCAGCTTTCTGG-3' (exon 6), yielding a 588-base pair (bp) product. Pggtlb was amplified as described by $(34,133)$ with primers, localized within the floxed segment (exon 7-9) sequences (cDNA, 5'-CCTTCTGTGGCATTGCGTCA-3' and 5'AGCCCATGCTGAAGT ATTAG-3'; product size, 600 bp). Threshold values were normalized to hypoxanthine 
phosphoribosyl transferase (Hprt) gene expression levels with primers 5'-

CAAACTTTGCTTTCCCTGGT-3' and 5'-CAAGGGCATATCCAACAACA-3' (250-bp product).

\section{Electroretinography (ERG)}

ERG was performed as described earlier $(59,106)$. Corneal evoked potentials were collected using UTAS-E4000 Visual Electrodiagnostic Test System and EMWIN 8.1.1 software (LKC Technologies).

\section{Immunoblotting}

Immunoblotting was performed on lash frozen retinal samples as described earlier $(59,106)$. The following antibodies were used: anti-PDE6 $\alpha$ (Thermo Fisher), anti-PDE6 $\beta$

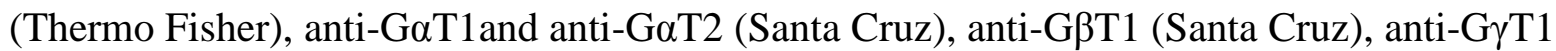
(Santa Cruz), anti-GyT2 (gift from Dr. Vadim Arshavsky, Duke University Eye Center, Durham, NC), anti-GRK1 (Thermo Fisher), anti-arrestin, RetGC1 (Affinity Bioreagents), and antiPDE6 $\alpha^{\prime}$ (3184P $(85,86)$. We used Odyssey goat anti-rabbit Alexa 680 and Odyssey goat antimouse Alexa 680 secondary antibodies (LI-COR Biosciences). Membranes were scanned with an Odyssey Infrared Imaging System (LI-COR Biosciences).

\section{Immunoprecipitation}

PDE6 assembly was assessed by IP with ROS-1 monoclonal antibody as described earlier $(86,106,120)$.

\section{TritonX-114 phase partitioning}


TritonX-114 phase partitioning was performed as described by (122). Briefly, flashfrozen retinal samples were homogenized in buffer $\mathrm{C}$ (20mM Tris-HCL, $\mathrm{pH} 7.5,0.5 \mathrm{mM} \mathrm{MgCl}$, $0.05 \mathrm{mM}$ EGTA, $0.5 \mathrm{mM}, \mathrm{NaN}_{3}$ and $150 \mathrm{mM} \mathrm{NaCl}$ ) by sonication with 5 pulses of 15 milliseconds at power setting 6 (Misonix XL-2000). Post centrifugation at $5000 \times g$ for 5 mins at $4{ }^{\circ} \mathrm{C}$, the supernatant was collected and cellular debris was discarded. Triton X114 was added in final concentration of $1 \%$, was gently mixed and incubated on ice for 5-10 mins. The samples were spun at $300 \times g$ for 5 mins at $37^{\circ} \mathrm{C}$ in swinging bucket centrifuge. Upper aqueous phase was separated and $1 \%$ tritonX114 was added to final volume. To the detergent phase, buffer C was added to match up volume to aqueous phase. Equal volumes of aqueous and detergent phase samples were loaded on gel and then analyzed by SDS-PAGE followed by immunoblotting as described previously.

\section{Membrane fractionation}

Cell fractionation was performed as described (59). All protein samples were then analyzed by SDS-PAGE followed by immunoblotting to check for the partitioning of cytosolic and membrane proteins.

\section{Immunohistochemistry}

Immunohistochemistry was performed as described by $(59,106)$. Mice were euthanized, and eyes were enucleated, fixed with $4 \%$ paraformaldehyde for 10 mins. Eyecups for cryosections were then fixed in paraformaldehyde in PBS solution before cryoprotection in $20 \%$ sucrose at $4{ }^{\circ} \mathrm{C}$ overnight. Eyecups were embedded in Tissue-Tek optimal cutting temperature compound (Sakura) and frozen in dry ice ethanol bath. Retinal blocks were sectioned with a 
Leica CM1850 Cryostat and $18 \mu \mathrm{m}$ sections were mounted on Superfrost plus slides.

Cryosections were washed in PBS solution, and then incubated in blocking buffer for $1 \mathrm{~h}$ followed by antibody staining as described.

\section{Transducin Translocation experiment}

For this experiment, the number of mice were divided in 3 different sets. For each set, three littermates and conditional knockout mice were used. Set 1: The mice were dark adapted and their eyes were enucleated under dark conditions; Mice were euthanized by $\mathrm{CO}_{2}$ inhalation, and their eyes were enucleated, fixed and cryo-sectioning was performed as described in previous section $(59,106)$. Set 2: Mice adapted to darkness were used for this experimental set up. The pupils of mice adapted to $24 \mathrm{~h}$ darkness were dilated as described by $(59,106)$ and mice were exposed to saturating light (1500 lux). Mice were euthanized by $\mathrm{CO}_{2}$ inhalation, and their eyes were enucleated, fixed with $4 \%$ paraformaldehyde and immunocytochemical analysis was performed. In Set 3, mice that were exposed to saturating light conditions were used for these experiments. Followed by exposure to bright light, these mice were dark reared for $2 \mathrm{~h}, 4 \mathrm{~h} 12 \mathrm{~h}$ and 24h. After dark rearing at each described time points, Mice were euthanized by $\mathrm{CO}_{2}$ inhalation, and their eyes were enucleated and immunocytochemical analysis was performed. 


\section{RESULTS}

\section{Retinal morphology is unaltered in the absence of protein prenylation}

To investigate the role of prenylation in retinal neurons, we eliminated Pggtlb (encoding the catalytic subunit of GGTase-I) or Fntb (encoding the catalytic subunit of FTase) using CreloxP recombination. The schemes used to generate photoreceptor-specific knockouts of Pggt $1 \mathrm{~b}$ or Fntb are illustrated in supplemental (Fig. 1 A \& F). Six3-Cre expression eliminates Pggtlb and Fntb in the retina and the ventral forebrain at embryonic day $9.5(59,106,145)$. We created Pggtlb conditional mice model by mating Pggt $1 b^{f l / w t}$ Six3-Cre males (hereafter referred to as

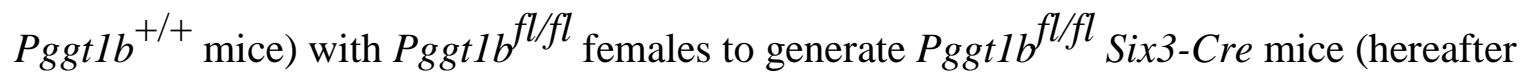
referred to as $\mathrm{Pggtlb}^{-/-}$mice, as well as littermate controls $\left[\right.$Pggtlb $^{f l / w t}$ Six3-Cre $\left(\right.$ Pggtlb $\left.^{+/+}\right)$; Pggtl $b^{f l f l}$, Pggtll $\left.\left.b^{f l / w t}\right)\right]$. For these studies, Pggtl $^{+/+}$littermates were used as controls; those mice which were similar to C57BL/6J animals with identical photoreceptor responses (not shown). Similar breeding strategy was used to generate Fntb conditional knockout mice (Fntb ${ }^{-1-}$ ) and littermate controls $\left[F n t b^{f l / w t}\right.$ Six3-Cre $\left.\left.\left(F n t b^{+/+}\right) ; F n t b^{f l / f l}, F n t b^{f l / w t}\right)\right]$. Quantitative RTPCR was performed $(34,146)$ to confirm the absence of Pggtlb and Fntb transcripts in the retina of Pggtlb $^{-/-}$and $\mathrm{Fntb}^{-/-}$mice (Fig. $1 \mathrm{D}$ and I). Polyclonal anti-CRE antibody was used for western blotting to verify the expression of CRE in $c r e$-positive mice lines $\left(\right.$ Pggtl $^{-/-}$and Fntb

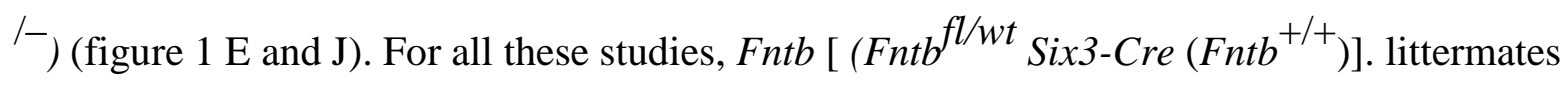
were used as controls. 
To study the effect of removal of prenyl transferases (Pggtlb or Fntb) on development of photoreceptors, we assessed the presence of rods and cones with cell-specific markers (Fig. $1 \mathrm{~B}$ and G). Rod and cone photoreceptor cells were present in a normal distribution in retinas of $\mathrm{Pggtlb}^{-/-}$and $\mathrm{Fntb}^{-/-}$mice as shown by the presence of peanut agglutinin (PNA, a marker for cone cells) and cyclic nucleotide gated channel (CNGA1/3, a marker for rod and cone cells) (Fig. $1 \mathrm{~B}$ and $\mathrm{G}$, top and bottom panels respectively). We also checked the retinal morphology by performing toluidine blue staining on retinal cryosections of Pggtlb and Fntb mice littermates. The retinal lamination was unaltered and we did not observe any changes in the thickness of the outer nuclear layer (ONL) at P18 (Fig. $1 \mathrm{C} \& \mathrm{H}$ ) Altogether, our data show that prenylation of proteins is not required for photoreceptor development.

\section{Light evoked ERGs are diminished in the absence of prenyl transferases}

We used electroretinography (ERG) to measure the photoreceptor function in Pggt1b $\left(\right.$ Pggtlb $^{+/+}$ and $\left.\mathrm{Pggtlb}^{-/-}\right)$and $\mathrm{Fntb}\left(\mathrm{Fntb}^{+/+}\right.$and $\left.\mathrm{Fntb}^{-/-}\right)$littermate mice. ERG is characterized by two wave fronts. The negative deflection, $a$-wave of scotopic ERGs is generated by hyperpolarization of rod photoreceptor cells in response to light. Subsequent signaling to downstream neurons leads to depolarization of post-synaptic bipolar cells, which is measured by the $b$-wave of ERGs, a positive deflection. In the mice lacking Pggtlb $\left(\mathrm{Pggtlb}^{-{ }_{-}}\right)$and $\mathrm{Fntb}\left(\mathrm{Fntb}^{-{ }_{-}}\right)$at P16, there were no significant changes in rod and cone mediated responses (Fig. 2 A and B left ERG traces). This finding is in agreement with our observation that photoreceptor development is normal in Pggtlb $\left(\right.$ Pggtlb $\left.^{-/-}\right)$and Fntb $\left(\mathrm{Fntb}^{-{ }_{-}}\right)$mice at P16 (Fig.1). However, as these mice age, rod responses progressively declined. At P25, in Pggtl $^{-1-}$ mice maximal rod responses 
were reduced by $93 \%(n=3$, Student's $t$-test, $P=0.002)$ and cone responses were reduced by $97 \%(n=3$, Student's $t$-test, $P=0.005)$. Similarly, at P60, in $F n t b^{-l-}$ mice maximal rod responses were reduced by $90 \%(n=3$, Student's $t$-test, $P=0.04)$ and cone responses were reduced by $95 \%(n=3$, Student's $t$-test, $P=0.002)$ (Fig. 2 A and B right traces). To see the age dependent decline in ERG function, the rod response at various ages were plotted (Fig. $2 \mathrm{C}$ and D). We also checked the sensitivity of ERG response at different light intensities in Pggtlb $\left(P_{g g t l b} b^{+/+}\right.$and $\left.P g g t l b^{-/-}\right)$and $F n t b\left(\mathrm{Fntb}^{+/+}\right.$and $\left.F n t b^{-/-}\right)$littermate mice (Fig. 2 E and F). Strikingly, we did not observe any significant changes in light sensitivity at P16 (not shown). However, at P25 in Pggtlb mice, and at P60 in Fntb mice, the sensitivity of ERG response showed a dramatic reduction. Collectively, these results show that rod and cone signal transduction pathways are disrupted in aged $\mathrm{Pggt} \mathrm{b}^{-/-} \mathrm{Fntb}-\mathrm{I}$ mice indicating the importance of prenylation for the photoreceptor function.

\section{Photoreceptors degenerate progressively in absence of Pggtlb or Fntb.}

To understand the reason behind progressive loss of ERG response, using propidium iodide (PI) as a marker for cell apoptosis, we checked for the age dependent degeneration in mice littermates lacking Pggtlb and Fntb (Fig. 3). We did not observe any changes in the thickness of the outer nuclear layer (ONL) at early developmental age i.e. P16 (Fig. 3 A \& E top panel). However, in the absence of Pggtlb, we observed the dramatic reduction in the thickness of the outer nuclear layer (ONL) at P25 (Fig. 3 B, right panel). The degenerating nuclei can be seen as bright spots. As the development progressed in the absence of Pggt $1 b$, ONL was completely diminished at the

age of P150 showing sever photoreceptor degeneration (Fig 3. B-D). In comparison to Pggt1b ${ }^{-/-}$ , the $\mathrm{Fntb}^{-\mathrm{I}}$ photoreceptors exhibited a slow and progressive loss of three to four nuclear layers 
by P150 (Fig.3 F- H). However, we did not observe any changes in inner nuclear layer (INL) or ganglion cell layer (GLC) (not shown) at all indicated ages. Altogether these results indicate that lack of protein prenylation in retina leads to progressive photoreceptor degeneration and is one of the major reasons behind loss of photoreceptor function.

\section{Lack of Pggtlb and Fntb in photoreceptors affects subset of phototransduction proteins}

We postulated that decreased ERG response in photoreceptors lacking protein geranylgeranylation or farnesylation may result from a reduction in the levels of one or more prenylated proteins involved in phototransduction pathway. Since we observed age dependent photoreceptor degeneration in mice lacking prenylation (Fig.3), it was necessary to analyze retinal protein levels prior to any signs of degeneration. Therefore, we assessed the protein levels by immunoblotting of the retinal extracts from Pggtlb $\left(\right.$ Pggtlb $^{+/+}$and Pgtlb $\left.^{-/-}\right)$and Fntb $\left(\mathrm{Fntb}^{+/+}\right.$and $\left.\mathrm{Fntb}^{-/-}\right)$littermate mice at P18 (before significant retinal degeneration was evident). In $P g g t 1 b^{-/-}$we observed more than a $20 \%$ reduction $(n=3$, Student's $t$-test, $P=$ 0.001) in levels of the catalytic rod PDE6 $\alpha \beta$ subunit and more than 40\% cone PDE6 (PDE6 $\alpha^{\prime}$ ) which are favored substrates of Pggtlb (Fig. 4 A and B). Surprisingly, we also observed significant reduction in levels of cone transducin GaT2, and G $\gamma \mathrm{T} 2$. As expected, rod arrestin (rARR), a photoreceptor protein that is not isoprenylated, was unaffected and serves as a control along with GAPDH. Similarly, in $\mathrm{Fntb}^{-1-}$ mice retinal extracts, we observed significant reduction in the levels of isoprenylated rod PDE6 catalytic subunits (PDE6 $\alpha \beta$ ) by 50\% and transducin complex ( $n=3$, Student's $t$-test, $P=0.05$ ). The transducin complex GaT1, and G $\beta 1$ G $\gamma \mathrm{T} 1$, and GRK-1 were also reduced in the absence of Fntb (Fig. $4 \mathrm{C}$ and D). Interestingly, 
known farnesylated protein, INPP5E (inositol polyphosphate-5-phosphatase) was severely affected (reduced by $70 \%$ ) in $\mathrm{Fntb}^{-/-}$retinae. Surprisingly, Rab28 another farnesylated protein (91) was unaffected in the absence of Fntb. Our results strongly suggest that lack of prenylation causes photoreceptor $C A A X$ proteins to undergo increased protein turnover, with transducin subunits, GRK1, PDE6, INPP5E showing the most pronounced reductions in their steady-state levels.

\section{Membrane association of subset of prenylated impaired in the absence of prenyl} transferases

The reason behind reduced protein levels could be because of defective membrane anchorage in the absence of prenylation. The addition of a lipid anchor to the $C A A X$ proteins is thought to increase hydrophobicity and provide firm membrane anchorage. Thus to examine the effects of geranylgeranylation and farnesylation on membrane attachment of phototransduction proteins, we performed isotonic cellular fractionation of retinal extracts from $P g g t 1 b\left(P g g t 1 b^{+/+}\right.$ and $\left.P g g t b^{-/-}\right)$and $F n t b\left(F_{n t b}{ }^{+/+}\right.$and $\left.F_{n t b} b^{-/-}\right)$littermate mice at P16. We found majority $(55 \%)$ of catalytic subunits of rod PDE6 $(\alpha \beta)$ localized in membrane fraction in retinal lysates from $P g g t 1 b^{+/+}$littermate controls $(\mathrm{n}=3, P<0.05)$. In contrast, less than $30 \%$ of rod PDE6 $(\beta)$ was present in membrane fraction in $\mathrm{Pggt} \mathrm{b}^{-/-}$. The membrane association of PDE6 $\alpha$, rod transducin, GRK-1 was not affected by the absence of Pggt1b. Guanylate cyclase-1 (RetGC1) and AIPL-1 were used as membrane-bound and cytosolic protein controls, respectively, and were unaffected by the deficiency in Pggt1b -mediated geranylgeranylation (Fig. 5 A and B). We also checked the membrane partitioning of several prenylated protein in mice lacking $\mathrm{Fntb}^{-/-}$. We 
found majority (more than $65 \%$ ) of catalytic subunits of rod PDE6 ( $\alpha \beta)$ to be localized in membrane fraction in retinal lysates from littermates $F n t b^{+/+}$mice $(\mathrm{n}=3, P<0.04)$. In contrast, less than $40 \%$ of rod PDE6 was present in membrane fraction in littermate control mice. Similarly, more than $70 \%$ of G $\gamma \mathrm{T} 1$ and GRK1 was localized to membrane fraction in retinal lysates from littermates $F n t b^{+/+}$mice. However, we observed only about $35 \%$ of G $\gamma \mathrm{T} 1$ and GRK1 were present in membrane fraction in $\mathrm{Fntb}^{-/-}$retinal extracts. Overall, our results demonstrate the lack of Pggtlb and Fntb results in impaired membrane attachment of prenylated proteins such as rod PDE6, GRK1 and transducin.

In order to determine whether prenylation of PDE6 and transducin is defective in Pggt1 $\mathrm{b}^{-/}$ mice, we examined retinal extracts from $\mathrm{Pggt}_{1 b^{-/}}$mice and $P g g t 1 b^{+/+}$littermate controls by Triton-X-114 phase partitioning (122), which segregates prenylated and membrane proteins into the detergent phase (DP) and cytosolic proteins into the aqueous phase (AP). In Pggt $1 b^{-1-}$ mice and $P g g t 1 b^{+/+}$littermate more than $96 \%$ of rod PDE6 $\alpha \beta(\mathrm{n}=3, P<0.05)$ was distributed in detergent phase but was found to be partitioned equally in the aqueous phase and detergent phase in $P g g t 1 b^{--}$mice $(P<0.05)$. The partitioning of farnesylated G $\gamma \mathrm{T} 1$, non prenylated G $\alpha \mathrm{T} 1$ and GaT2 were unaffected as compared to littermate controls. These results show that Pggtlbmediated protein geranylgeranylation is required for proper membrane attachment of rod PDE6.

Similarly, in the Fntb littermate mice, more than $98 \%$ of PDE6 $\alpha$ and PDE6 $\beta$ (n=3, $P<0.05$, bottom panel) was present in detergent phase however was found to be partitioned equally in the aqueous phase and detergent phase in $F n t b^{-1-}$ mice $(P<0.05)$. More interestingly, $59 \%$ of the farnesylated $\mathrm{G} \gamma \mathrm{T} 1$ was in the aqueous phase $(P<0.02)$ (Fig. 6 bottom panel), whereas in the controls, $97 \%$ of G $\gamma \mathrm{T} 1$ was in the detergent fraction. This illustrates that farnesylation of PDE $\alpha$ and $\mathrm{G} \gamma \mathrm{T} 1$ are crucial for efficient membrane association 


\section{Transducin is mislocalized to the IS in the absence of farnesylation}

The G-protein transducin $\gamma(\mathrm{G} \gamma \mathrm{T} 1)$ subunit is known farnesylated protein $(36,74)$. Consistent with earlier reports, our membrane fractionation data suggest that lack of protein farnesylation affects the membrane anchorage of $\mathrm{G} \gamma \mathrm{T} 1$. Farnesylation of $\mathrm{G} \gamma \mathrm{T} 1$ is thought to be involved in proper localization of protein to rod OS (74). Here we investigated the localization of G-protein transducin complex in the absence of Fntb and Pggtlb (Fig. 7). We performed immunocytochemistry on retinal cryosections using antibodies specific for individual subunits of transducin complex G $\alpha \mathrm{T} 1, \mathrm{G} \beta 1$ and $\mathrm{G} \gamma \mathrm{T} 1$. These retinal samples for cryosectioning were prepared under ambient light conditions. As expected, in Fntb littermate controls $\left(F n t b^{+/+}\right)$, majority of transducin G $\alpha \mathrm{T} 1, \mathrm{G} \beta 1$ and $\mathrm{G} \gamma \mathrm{T} 1$ (about 80\%, $\mathrm{n}=3, \mathrm{p}<0.05$ ) was localized to the rod

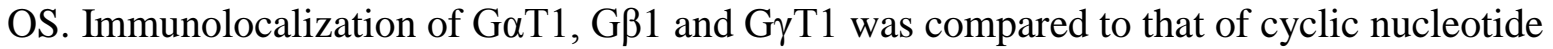
gated channel (CNGA1/3), a marker of OS (Fig. 7 A-C, top panels). Merged images demonstrated that transducin was transported to the OS and co-localized with CNGA1/3 in Fntb

littermate controls $\left(F n t b^{+/+}\right)$. However, to our surprise, we observed significant amounts (more

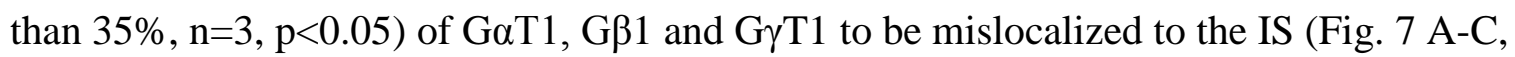
bottom panel) in mice lacking Fntb. Such transducin mislocalization were not observed in Pggtlb deficient mice (Fig.7 D-F). Note that at tested ages the OS lengths are shorter in both $\operatorname{Pggtlb}^{-/-}$and $\mathrm{Fntb}^{-/-}$as compared to their littermate controls $P g g t 1 b^{+/+}$and $\mathrm{Fntb}{ }^{+/+}$due to degeneration.

\section{Lack of Fntb affects the reappearance of translocated transducin to rod outer segments}


To understand the possible mechanism underlying the mislocalization of transducin (Fig.7) in $\mathrm{Fntb}^{-1-}$ mice, we investigated the light-dependent transducin translocation and re-entry of translocated transducin to rod OS using immunohistochemistry $(13,68,74,142)$ under dark adapted, saturating lighting condition (1500 lux) and recovery conditions post light saturation (2h, 4h,12h and 24h). In the 24h dark-adapted state, both GaT1 (not shown) and G $\gamma \mathrm{T} 1$ immunoreactivities were predominantly detected in the outer segment layer of $\mathrm{Fntb}\left(\mathrm{Fntb} \mathrm{H}^{+/+}\right.$ and $\mathrm{Fntb}^{-/-}$) littermate mice (Fig.8 I; A). After exposing to the saturating light conditions for 15 mins, more than $75 \%(\mathrm{n}=3, \mathrm{P}<0.05)$ of $\mathrm{G} \alpha \mathrm{T} 1$ (not shown) and $\mathrm{G} \gamma \mathrm{T} 1$ in $F n t b\left(F n t b^{+/+}\right.$and $F n t b^{-}$ ${ }^{-}$) littermate mice translocated to the inner regions of the photoreceptor layer, including the inner segment, the perinuclear region, and the synaptic terminal (Fig.8 I; B). After light saturation, $\mathrm{Fntb}\left(\mathrm{Fntb}^{+/+}\right.$and $\left.\mathrm{Fntb}{ }^{-/-}\right)$littermate mice were dark adapted for different time periods (recovery conditions) such as 2h, 4h,12h and 24h (Fig.8 I; C, D and E). After $2 \mathrm{~h}$ of dark adaptation, in $\mathrm{Fntb}^{+/+}$, the translocated transducin G $\gamma \mathrm{T} 1$ re-appears in the OS (Fig.8 I; C, left panel) illustrating normal return kinetics of translocated transducin. In contrast, in the Fntb ${ }^{-1-}$ mice retina, more than $70 \%$ of $\mathrm{G} \gamma \mathrm{T} 1(\mathrm{n}=3, \mathrm{P}<0.05)$ immunoreactivity were detected in these inner regions after $2 \mathrm{~h}$ of dark adaptation (Fig.8 I; C, left panel and III) representing the slow reentry of translocated $\mathrm{G} \gamma \mathrm{T} 1$ from inner regions of the photoreceptor layer to OS. Interestingly, up to $12 \mathrm{~h}$, translocated G $\mathrm{TT} 1$ immunoreactivity was detected in these inner regions in $\mathrm{Fntb}^{-1-}$ mice retina (Fig.8 I; D and III) showing defects in re-transport of translocated G $\gamma$ T1 to OS. We observed similar defects in GaT1 translocation (not shown). Finally, after 24h we observed the complete accumulation of G $\gamma \mathrm{T} 1$ to OS in $\mathrm{Fntb}^{-/-}$mice retina (Fig.8 I; E and III). The movement 
of rod arrestin (rARR) appeared to be normal under different light and dark conditions (not shown). Such defects were not detected in the mice lacking Pggtlb (Fig.8 I; F, G and H), which strongly suggests that the lack of farnesylation affects the re-appearance kinetics of translocated transducin to rod OS.

\section{Levels of rod PDE6 and GRK-1 are severely affected in the absence of Pggt1b or Fntb}

The lipid anchors are thought to be involved in transport of prenylated proteins to OS. Thus we examined the trafficking of rod PDE6 and other isoprenylated OS proteins in Pggtlb or Fntb deficient mice (Fig. 9). Due to lack of good antibody for PDE6 $\alpha$ subunit, we used MOE antibody that detects. both Rod and cone PDE6 catalytic subunits. In retinal extracts from Pggtlb $\left(\right.$ Pggtlb $^{+/+}$and Pggtlb $\left.^{-/-}\right)$and $\mathrm{Fntb}\left(\mathrm{Fntb}^{+/+}\right.$and $\left.\mathrm{Fntb}^{-/-}\right)$littermate mice at indicated ages, we compared the immunolocalization of PDE6 to that of cyclic nucleotide gated channel (CNGA1/3), a marker of OS (Fig. 9 A and B respectively). Merged images demonstrated that rod PDE6 was transported to the OS and co-localized with CNGA1/3 in retinas lacking Pggtlb or Fntb expression. G-protein coupled receptor kinase 1 (GRK1), a farnesylated protein, is

expressed in both rod and cone photoreceptor cells. In Pggtlb littermate controls $\left(\right.$ Pggtl $\left.^{+/+}\right)$, GRK-1 was present in rod and cone photoreceptors, Interestingly, in Pggtlb deficient mice, GRK-1 was severely reduced in cones while expression of GRK-1 in rods did not show any significant changes (Fig. $9 \mathrm{C}$ arrow heads). This result is consistent with our findings with cone specific knockout of Pggtlb. Since GRK-1 is farnesylated, in Fntb deficient mice, the proteins levels were drastically reduced as can be observed with faint staining of GRK-1 in rods and cones (Fig. 9 D). However, the GRK-1 in cones was present in detectable amounts (Fig. 9 D, arrow heads). These results highlight the importance of protein geranylgeranylation in expression 
of GRK1 in cones. Overall, our results show that prenylation of proteins has little or no effect on the ability of isoprenylated rod PDE6 to traffic to the OS but it is crucial for the stability of isoprenylated OS proteins such as PDE6 and GRK-1.

\section{Rod PDE6 assembly is not affected in the photoreceptors lacking Pggt1b and Fntb}

We wanted to investigate if assembly of heteromeric rod PDE6 $\alpha \beta$ is affected by the lack of prenylation in our animal models. As described earlier, the od PDE6 is differentially prenylated, PDE6 $\alpha$ being farnesylated and PDE6 $\beta$ being geranylgeranylated. Previously we have shown that geranylgeranylation of cone PDE6 catalytic subunits is crucial for its assembly in cone photoreceptors (Pendse ND et al, unpublished findings). To examine the assembly of rod PDE6, we immunoprecipitated PDE6 with ROS-1, a monoclonal antibody that recognizes the assembled PDE6 (84,123). Immunoprecipitated proteins were separated on SDS/PAGE gels followed by immunoblotting using polyclonal antibody that recognizes the catalytic subunit of

rod PDE6. To our surprise, we observed that in the absence of Pggtlb $\left(\right.$ Pggtlbt $\left.^{-1-}\right)$ or Fntb $\left(\mathrm{Fntb}^{+/+}\right.$and $\left.\mathrm{Fntb}^{-/-}\right)$, ROS-I antibody can still interact and pull down catalytic subunits of rod PDE6, suggesting that removal of single lipid anchors still retains the ability of rod PDE6 to assembly properly (Figure $10, \mathrm{n}=3, P<0.05$ ). IP with non-specific mouse $\mathrm{IgG}$ serving as experimental control. In conclusion, we find that the presence of a single lipid anchor, either farnesyl or geranylgeranyl, is sufficient for assembly of rod PDE6. 


\section{DISCUSSION}

In the present investigation, we show the importance of prenylation of proteins in the function and survival of photoreceptor neurons. Most striking defect in mice lacking prenylation in the retina is the loss of visual function accompanied by progressive photoreceptor degeneration. Lack of prenylation resulted in defective association of prenylated transducin and PDE6 with photoreceptor membranes and resulted in their decreased protein levels. While the assembly and trafficking of rod PDE6 was unaffected in the absence of prenylation, the rod transducin localization and translocation kinetics were severely affected in the absence of Fntb. Such transducin localization and translocation defects were not observed in the absence of protein geranylgeranylation in rods.

\section{What triggers retinal degeneration and loss of ERG function in mice lacking prenylation in retina?}

Interestingly, despite of absence of Fntb or Pggt1b, we did not observe any signs of retinal degeneration or photoreceptor cell death at early stages P16. The development of photoreceptors progressed normally and we observed no defects in photoreceptor development, morphology and abundance at P16, well after the Cre mediated excision of Fntb or Pggtlb is complete (E9.5). It appears that photoreceptors do not need protein farnesylation or geranylgeranylation for its survival at early developmental stages (P16). However, in the absence of prenylation, the photoreceptor showed dramatic reduction in rod and cone mediated ERGs demonstrating the need for prenylation in functioning of photoreceptors. The characteristic layering of neurons in the retina was preserved, and all retinal layers were formed normally. 
These findings are in agreement with normal photoreceptor ERG response at P16. However, we observed progressive reduction in rod and cone responses with extinguished photoreceptor responses over the time.

It is commonly accepted that in all forms of retinal degeneration, photoreceptors eventually die via apoptosis, but the molecular events triggering cell death are specific to each degeneration type and range from abnormal light signaling to protein mislocalization and misfolding $(147,148)$. One of the striking observations in the mice lacking prenylation was reduced light evoked response. The ERG response generated by mice lacking prenylation showed dramatic reduction in the amplitude of "a" and " $b$ " waves at later stages of development (P25) and the response was absent at P60. The major cause of retinal degeneration and loss of ERG function in mouse model lacking farnesylation is reduced stability and membrane association of farnesylated proteins such as transducin gamma (G $\gamma \mathrm{T} 1)$, rod PDE6 $\alpha$ and GRK1. Interestingly a farnesylated protein INPP5E, that is localized to cilium (149) was reduced dramatically at P16 in mice lacking farnesylation. INPP5E is needed for normal photoreceptor function and ciliogenesis (150-152). We believe that lack of farnesylation of INPP5E results in decrease in its steady state levels and is one of the major causes behind photoreceptor degeneration in mice lacking Fntb. (149) More strikingly we observed the mislocalized transducin complex and the impaired light dependent transducin translocation in mice retina lacking farnesylation. We believe this is the one of the primary cause of progressive retinal degeneration in mice retina lacking farnesylation. Interestingly in the mice retina protein geranylgeranylation, although transducin localization and translocation was not affected, we observed major reduction in the membrane association and protein levels of PDE6 $\beta$ which is a known target for protein geranylgeranylation and its binding partner PDE6 $\alpha$ subunit, cone PDE6 
which is though to be geranylgereanylated and cone transducin. Moreover, we observed similar reduction in cone GRK1 levels in mice lacking Pggtlb. Our previous study demonstrates that lack of Pggltb cones results in defective assembly of cone PDE6 and reduced stability of cone transducin, RetGC1 and GRK1. We believe the major reduction in the membrane association and protein levels of rod PDE6, a key phototransduction enzyme, is the root cause of retinal degeneration in Pggtlb lacking.

\section{Why transducin returns slowly to OS in mice lacking $F$ ntb?}

Rod transducin is a trimer composed of $\mathrm{G} \alpha \mathrm{T} 1$ which is myristoylated, non lipidated $\mathrm{T} \beta$ and $\mathrm{T} \gamma$ which is farnesylated(36,139). Acylated G $\alpha \mathrm{T} 1$ and prenylated $\mathrm{G} \beta \gamma$ most likely combine to form heterotrimeric $\mathrm{G}$ proteins at the endoplasmic reticulum in photoreceptor IS $(12,29,113)$. Light-activation of rhodopsin triggers GTP/GDP exchange on T $\alpha$, causing T $\alpha$-GTP and G $\beta \gamma$ to dissociate and traffic to the inner segment by passive. On arrival at the inner segment following

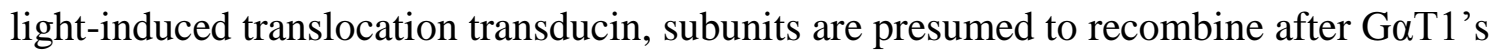
intrinsic GTPase activity hydrolyzes GTP, permitting heterotrimeric transducin to dock to inner segment membrane. On return to the dark, both $\mathrm{G} \alpha$ and $\mathrm{G} \beta \gamma$ subunits return to the outer segments in hours in wild-type mice. It will take approximately $2.2 \mathrm{hrs}$ for transducin to return to the outer segment, which agrees with experimentally observed return rates to rod OS $(153,154)$. Our results indicate that the deletion of farnesyl transferases in mouse leads to partial retention of transducin in the inner segment under ambient light conditions. The translocation of G $\gamma \mathrm{T} 1$ should contribute to the decrease in rod sensitivity, because declined level of G $\gamma \mathrm{T} 1$ in the outer segment should reduce the efficiency of GaT1 coupling (75). We believe that the myrestoyl

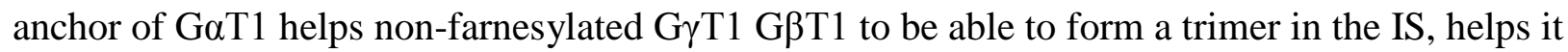


to dock on a transport vesicle and transport to OS. Under saturating light conditions, non farnesylated G $\gamma \mathrm{T} 1 \mathrm{G} \beta \mathrm{T} 1$ dissociats from rod OS and tanslocates to IS. For effecient return of

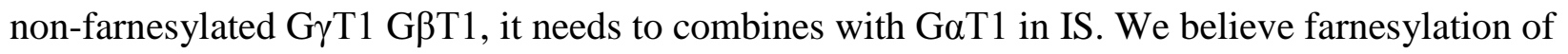
$\mathrm{G} \gamma \mathrm{T} 1$ expedites this process.However its absence may result in slow return of G $\gamma \mathrm{T} 1$ to rod OS. Our model is consistent with abnormal localization of transducin in other types of mutants that express non-prenylated $\mathrm{G} \gamma \mathrm{T} 1$ in rod cells.In these mutants, $\mathrm{G} \gamma \mathrm{T} 1$ expresses high solubility and is diffused to the inner region of rod cells demonstrating the unique role of farnesylation for proper redistributuion of transducin.

\section{Why does Rod PDE6 assemble in the absence of prenylation?}

Our previous studies have shown that in the absence of aryl hydrocarbon receptor interacting protein like 1 (AIPL1), PDE6 subunits are synthesized normally but are not stable and fail to assemble properly. Misassembled PDE6 is likely to be degraded by proteasome machinery in the IS of photoreceptors leading to decreased protein levels of PDE6 $(84-87,120)$. More recently, we have shown that geranylgeranylation of cone PDE6 catalytic subunits is crucial for its assembly in cone photoreceptors. We further demonstrated the geranylgeranyl lipid anchors on cone PDE6 acts as a "molecular grip" to facilitate either the interaction of cone PDE6 with AIPL1 or PDE6 assembly, a step needed for synthesis of functional PDE6 in cones (Pendse ND et al. 2016 unpublished findings). Rod PDE6 is differentially prenylated. In the absence of Pggtlb or Fntb, rod PDE6 subunits retain a single lipid anchor which we believe is sufficient for efficient interaction of PDE6 with AIPL1. Thus allowing efficient and proper assembly of PDE6

Overall, the current investigations revealed that prenylation is essential for photoreceptor function and survival. 


\section{FIGURE LEGENDS AND FIGURES}

Figure 1. Retinal morphology is unchanged in the absence of protein prenylation

(A \& F) Schematics for generating retina specific conditional knockouts for Pggt $1 b$ and Fntb. (B) Cryosections of P18 Pggtlb ${ }^{+/+}$(left) and Pggt1b $^{-/-}$(right) littermate mice labeled with peanut agglutinin (PNA-green, top panel), cyclic-nucleotide gated channel alpha 1/3 (CNGA1/3 -red, bottom panel), and DAPI (blue). OS, Outer Segment; IS, Inner Segment; ONL, Outer Nuclear Layer. Scale $=5 \mu \mathrm{M}$. (C) Cryosections stained with toluidine blue P18 Pggt1b ${ }^{+/+}{ }_{(\text {left })}$ and $\operatorname{Pggtlb}^{-/-}$(right) littermate mice showing intact retinal lamination, OS, Outer Segment; IS, Inner Segment; ONL, Outer Nuclear Layer; INL, inner nuclear layer. (D) RT-PCR analysis of P10 retinal cDNA showing the expression of Pggtlb (normalized to GAPDH) $(n=3$, Student's $t$-test, $P=0.0039$ ). ( $\mathbf{E}$ and $\mathbf{J})$ Immunoblots probed with CRE-specific antibody shows CRE expression only in CRE-positive mice lines $\left(P_{g g t} b^{-/-}, F n t b^{-/-}\right)$. GAPDH was used as a loading control. (G) Cryosections stained with toluidine blue P18 Fntb ${ }^{+/+}$(left) and Fntb ${ }^{-/-}$ (right) littermate mice showing intact retinal lamination. (H) Cryosections stained with toluidine blue P18 Fntb ${ }^{+/+}$(left) and $F n t b^{-/-}$(right) littermate mice showing intact retinal lamination. (I) RT-PCR analysis of P10 retinal cDNA showing the expression of Fntb (normalized to GAPDH) ( $n=3$, Student's $t$-test, $P=0.0041)$. 

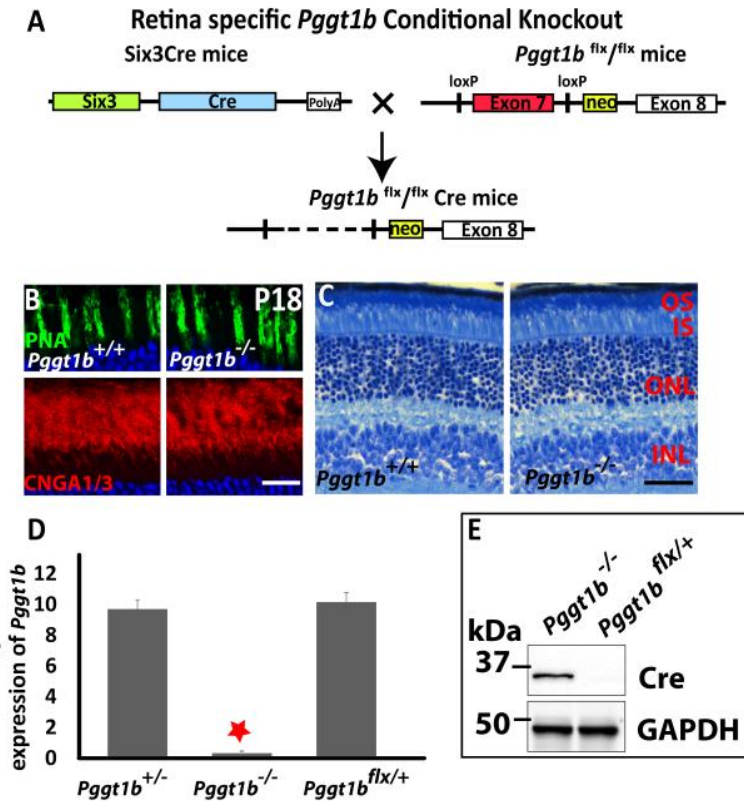

F Retina specific Fntb Conditional Knockout
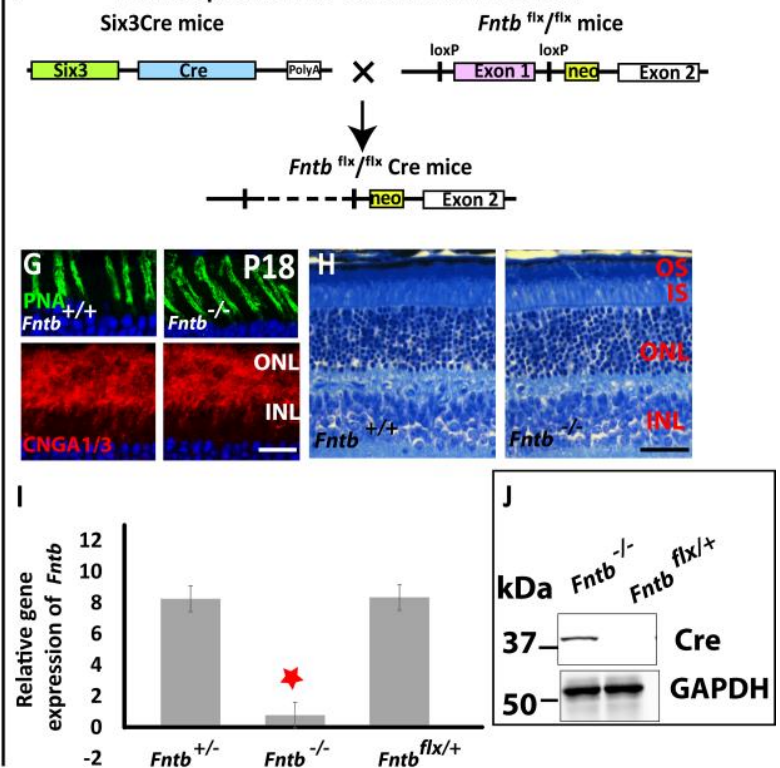

Figure. 1 Retinal morphology is unchanged in the absence of protein prenylation 
Figure 2. Light evoked ERGs are progressively diminished in the absence of prenyl transferases

(A and B) Representative waveforms of rod and cone ERG responses recorded from mice lacking Pggtlb (A) and Fntb (B) with their littermate controls at indicated ages. (C and D) Amplitude of "a" wave versus age (in days). Rod responses recorded from mice lacking Pggtlb (C) and Fntb (D) correspond to the amplitude of the "a" wave. Representative rod waveforms measured at $-0.8 \log \mathrm{cd}^{*} \mathrm{~s} / \mathrm{m}^{\wedge} 2$, while representative cone waveforms measured at $0.7 \log$ $\mathrm{cd}^{*} \mathrm{~s} / \mathrm{m}^{\wedge} 2(n=3) . \mathrm{P}$, postnatal days; $\mathrm{ms}$, milliseconds; $\mu \mathrm{V}$, microvolts; $n$, number of littermates. (E and F) Intensity response relations of scotopic "a" waves from mice lacking Pggtlb (E) and Fntb (F) with their littermate controls at indicated ages $(n=3)$. The data were fitted with the hyperbolic functions that yielded scotopic "a" wave half-saturating light intensities of $0.08 \pm$ $0.01 \mathrm{~cd}^{*} \mathrm{sm}^{-2}$ for $P g g t l b^{+/+}$and $0.17 \pm 0.022 \mathrm{~cd}^{*} \mathrm{sm}^{-2}$ for $P g g t l b^{-/}$littermates respectively at

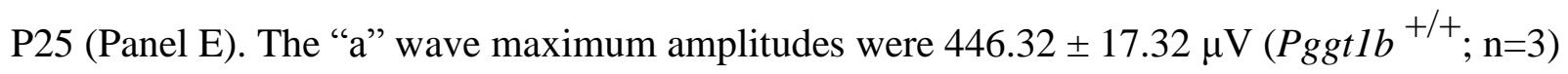
and $81.79 \pm 10.11 \mu \mathrm{V}\left(\right.$ Pggtlb $\left.^{-/-} ; \mathrm{n}=3\right)$. Similarly, for $\mathrm{Fntb}^{-/-}$littermates respectively at P60, data were fitted with the hyperbolic functions that yielded scotopic "a" wave half-saturating light intensities of $0.12 \pm 0.02 \mathrm{~cd}^{*} \mathrm{sm}^{-2}$ for $\mathrm{Fntb}{ }^{+/+}$and $0.11 \pm 0.020 \mathrm{~cd}^{*} \mathrm{sm}^{-2}$ for $\mathrm{Fntb}^{-/-}$littermates respectively at P60 (Panel F). The "a" wave maximum amplitudes were $434.37 \pm 19.74 \mu \mathrm{V}$ (Fntb $\left.{ }^{+/-} ; \mathrm{n}=3\right)$ and $62.41 \pm 14.31 \mu \mathrm{V}\left(\right.$ Fntb $\left.^{-/-} ; \mathrm{n}=3\right)$. The values are mean $\pm \operatorname{SEM}(* *=P<0.05)$ 
A

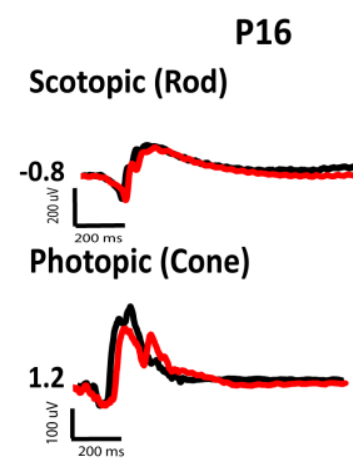

B
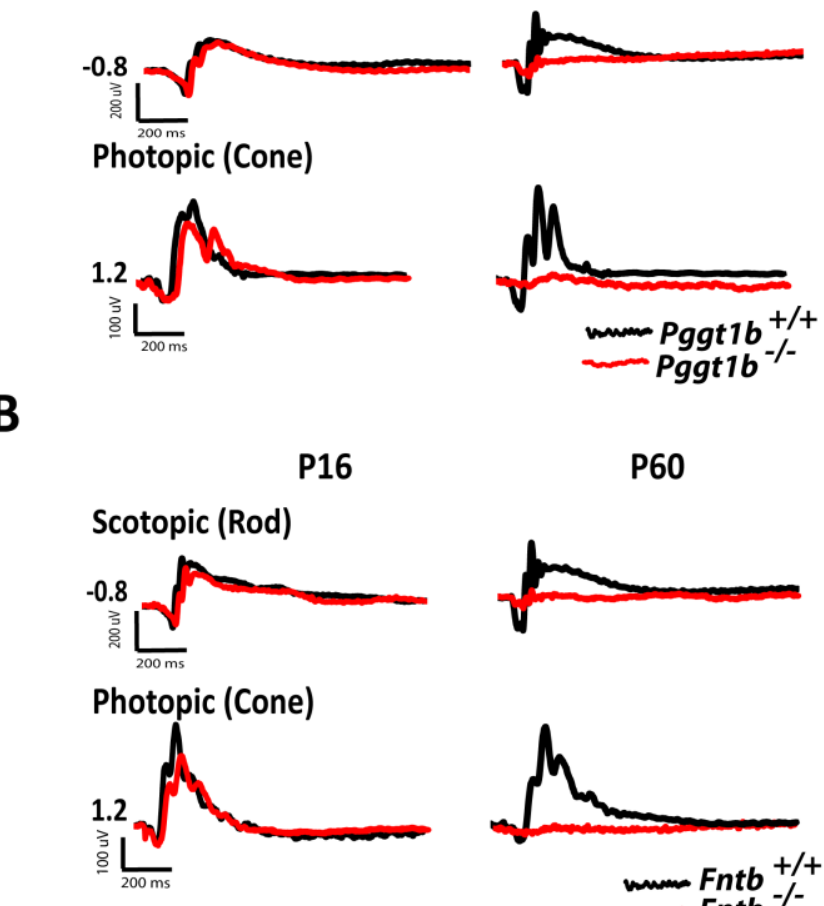

D

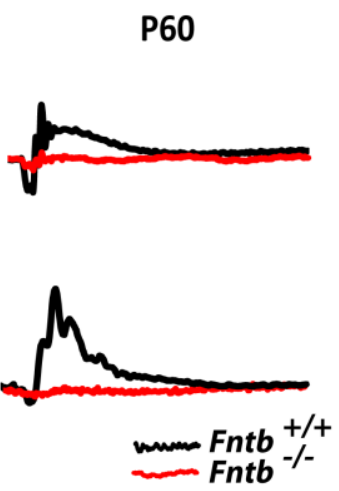

E

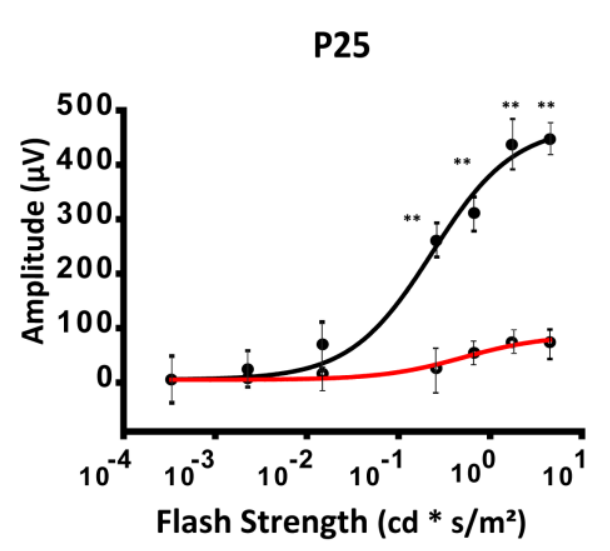

C
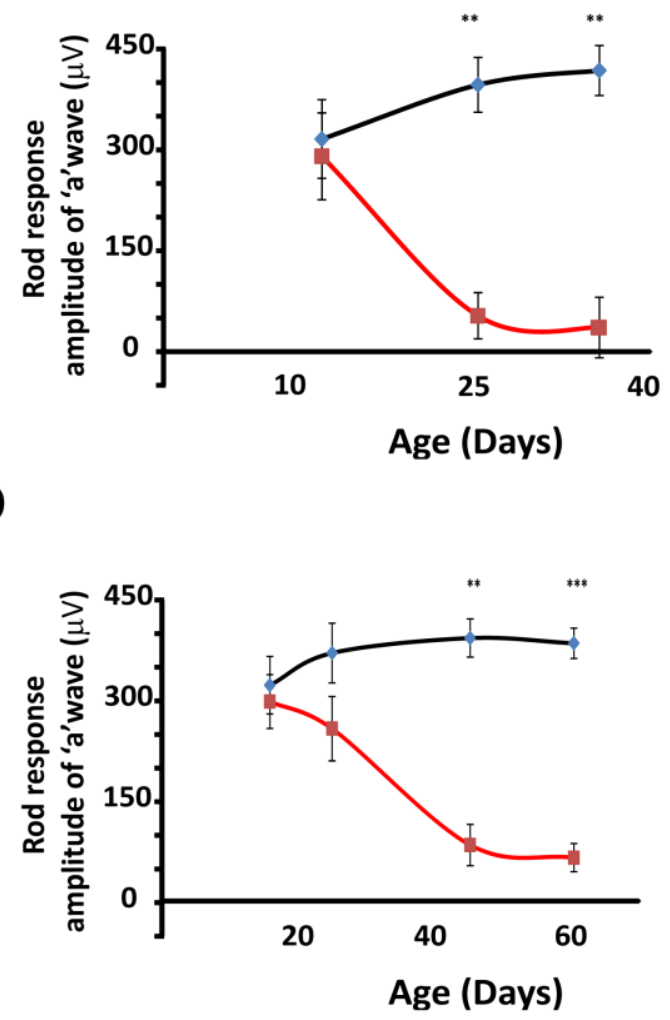

$\mathbf{F}$

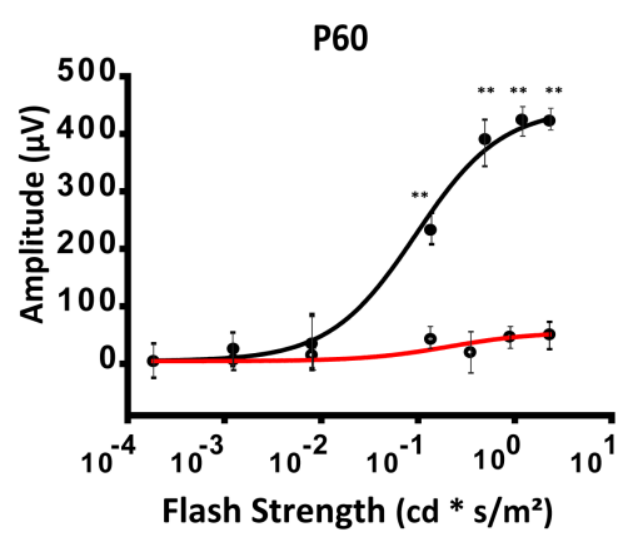

Figure. 2 Light evoked ERGs are diminished in the absence of prenyl transferases 
Figure 3. Photoreceptors degenerate progressively in absence of Pggt1b or Fntb

(A-D) Cryosections of Pggtlb $^{+/+}$(left) and $\operatorname{Pggtlb}^{-/-}$(right) littermate mice at all indicated ages, labeled with propidium iodide (PI-red), a nuclear stain. (E-H) Cryosections of $F n t b{ }^{+/+}$ (left) and $\mathrm{Fntb}^{-{ }^{-}}$(right) littermate mice at all indicated ages, labeled with propidium iodide (PI), a nuclear stain. ONL, Outer Nuclear Layer; INL, inner nuclear layer. 


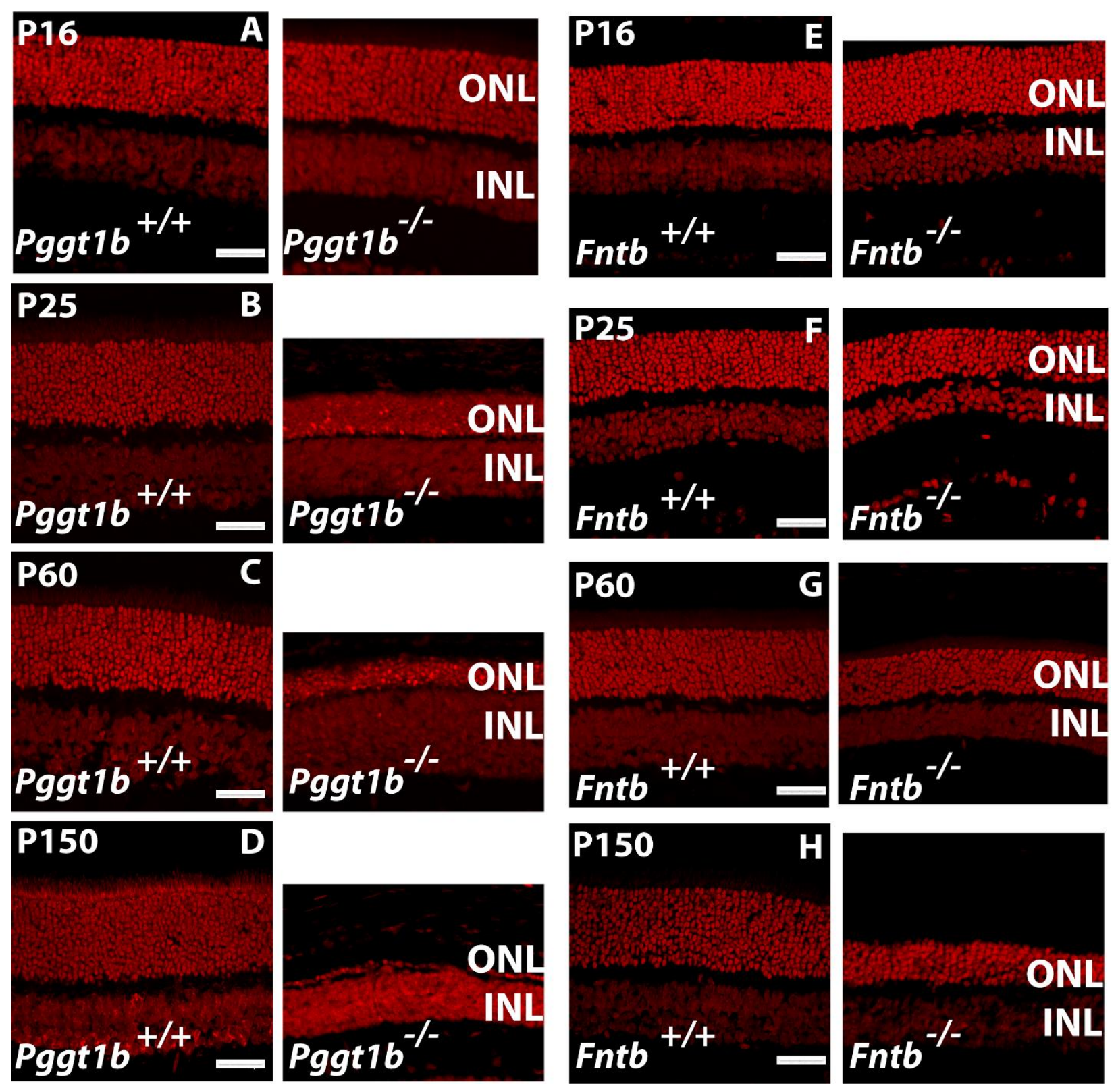

Figure. 3 Photoreceptors degenerate progressively in absence of Pggt1b or Fntb 
Figure 4. Lack of Pggt1b and Fntb in photoreceptors affects subset of phototransduction proteins

(A) Representative immunoblots of retinal protein extracts from Pggtlb deficient mice $\left(\right.$ Pggtl $\left.^{-/-}\right)$and littermate controls $\left(\right.$Pggtlb $\left.^{+/+}\right)$at P18, probed with antibodies against indicated proteins. (B) Quantitative comparison of indicated photoreceptor protein levels derived from immunoblots shown in Panel A. Integrated intensity values were normalized to GAPDH $(n=3$, Student's $t$-test; $* * P<0.05)$. (C) Immunoblots performed similar to Panel A. Retinal extracts from P18 Fntb-deficient mice $\left(\mathrm{Fntb}^{-/-}\right)$and littermate controls $\left(\mathrm{Fntb}{ }^{+/+}\right)$were used. (D) Quantitative comparison of indicated photoreceptor protein levels derived from Panel $\mathrm{C}(n=3$, Student's $t$-test; $* * P<0.05)$. 


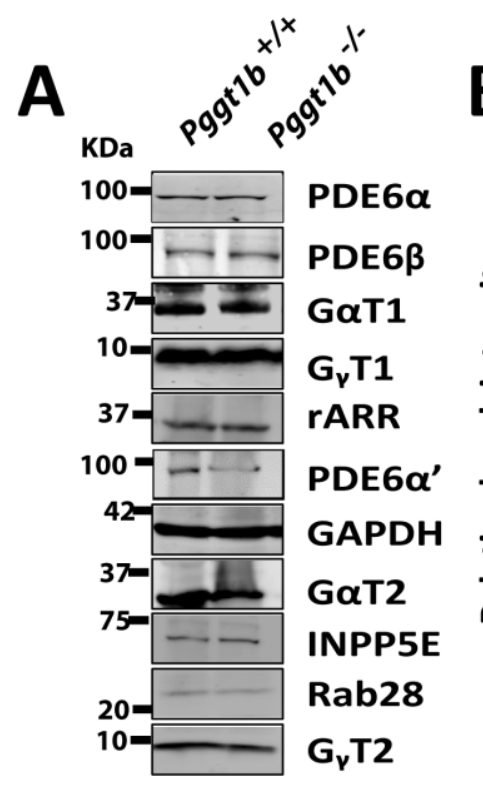

B $\quad P^{\prime} g g t 1 b^{+/+} \backsim P g g t 1 b^{-/-}$

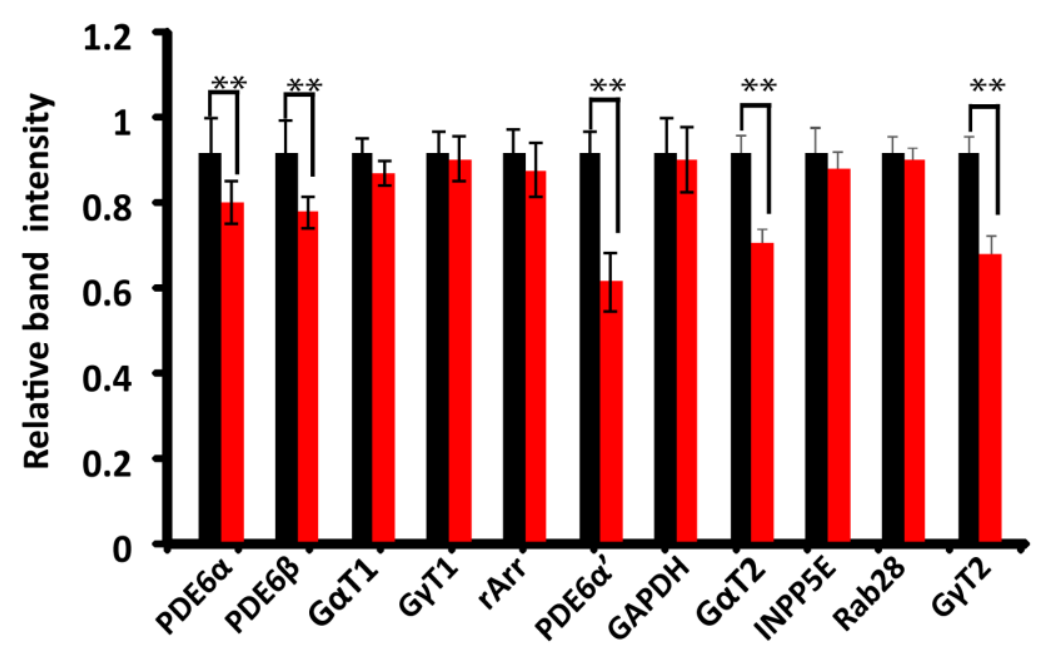

C

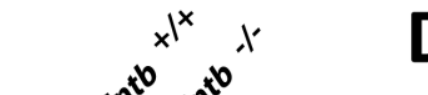

D

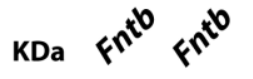

$\square \mathrm{Fntb}^{+/+}=\mathrm{Fntb}^{-/-}$

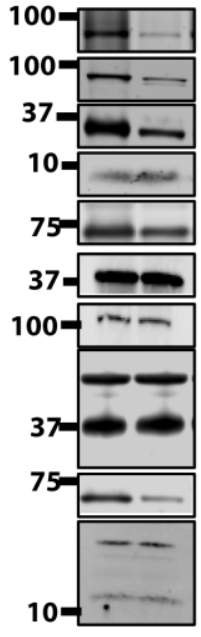

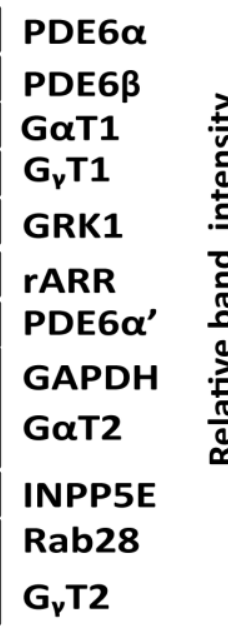

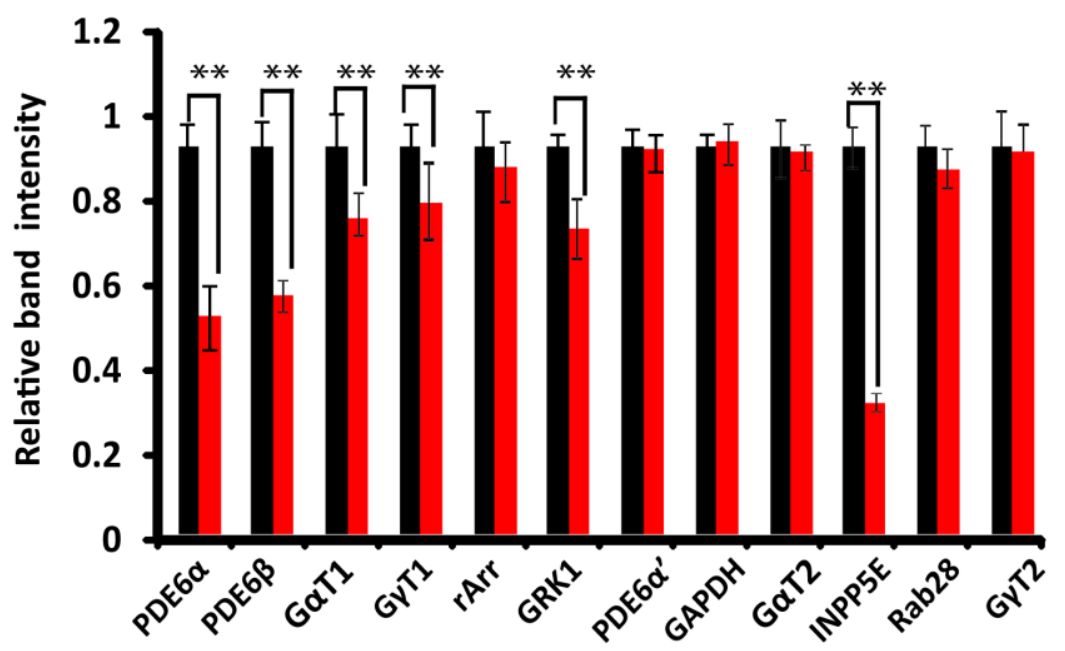

Figure. 4 Lack of Pggtlb and Fntb in photoreceptors affects subset of phototransduction proteins 


\section{Figure 5. Membrane association of subset of prenylated impaired in the absence of prenyl transferases}

(A) Isotonic cellular fractionation of P16 retinal lysates from Pggtlb deficient mice $\left(\right.$ Pggtl $\left.^{-1-}\right)$ mice and littermate controls $\left(\right.$ Pggtl $\left.^{+/+}\right)$, followed by immunoblotting with indicated antibodies. RetGC-1, AIPL1 serve as controls $(n=4)$. (B) Quantitation of western blots from Panel A. $\left(n=3,{ }^{* *} P<0.05\right.$, red bar, membrane fraction, black bar, cytosolic fraction; +/+, Pggtlb $^{+/+} ;-/-$, Pggtlb $^{-/-} \mathrm{T}=$ total fraction; $\mathrm{C}=$ cytosolic fraction; $\mathrm{M}=$ membrane-bound fraction). (C) Isotonic cellular fractionation of P16 retinal lysates from Fntb deficient mice (Fntb ${ }^{-/-}$mice and littermate controls $\left(\right.$Fntb $^{+/+}$, followed by immunoblotting $(n=3)$. (D) Quantitation of western blots from Panel C. $(n=3, * * P<0.05$; red bar, membrane fraction, black bar, cytosolic fraction; +/+, Fntb ${ }^{+/+} ;-/-$, Fntb $^{-/-}$). 

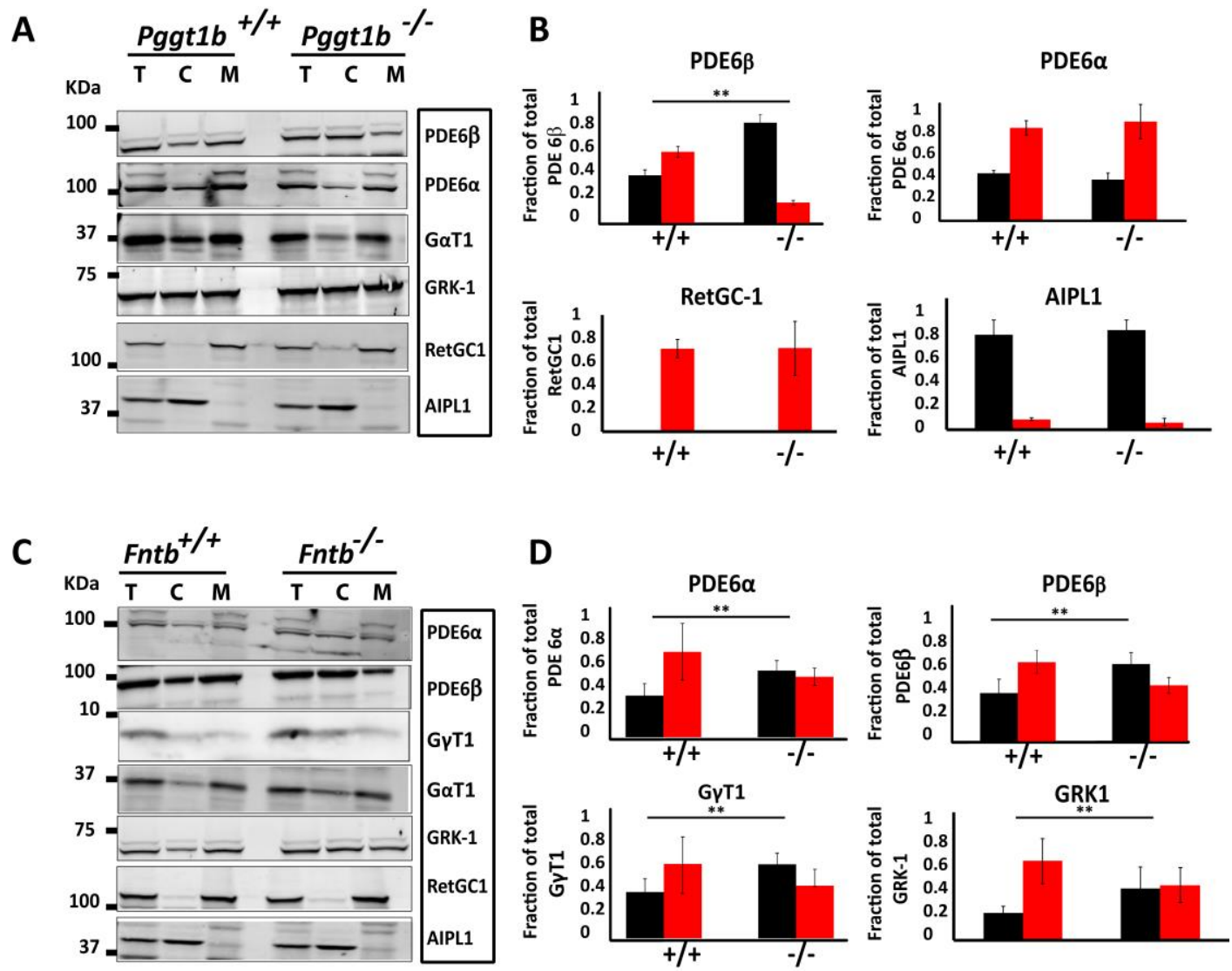

Figure. 5 Membrane association of sub-set of prenylated impaired in the absence of prenyl transferases 
Figure 6. Defective partitioning of phosphodiesterase and transducin- $\gamma$ subunit in Triton X114 detergent in the absence of prenylation. (A) Triton X-114 phase partitioning of P16 retinal lysates from $\mathrm{Pggtl}^{-/-}$and $\mathrm{Fntb}^{-/-}$mice and their respective $\mathrm{Pggtlb}^{+/+}$and $\mathrm{Fntb} \mathrm{b}^{+/+}$littermate controls, followed by immunoblotting. (B) Quantitation of western blots from panel A, $n=3$, $* * P<0.05 .+/+=P g g t 1 b^{+/+}$or $\mathrm{Fntb}^{+/+} ;-/-=\mathrm{Pggtl}^{-/-}$. Or Fntb ${ }^{-/-}$. AP, aqueous phase (red bar); DP, detergent phase (black bar). 
A
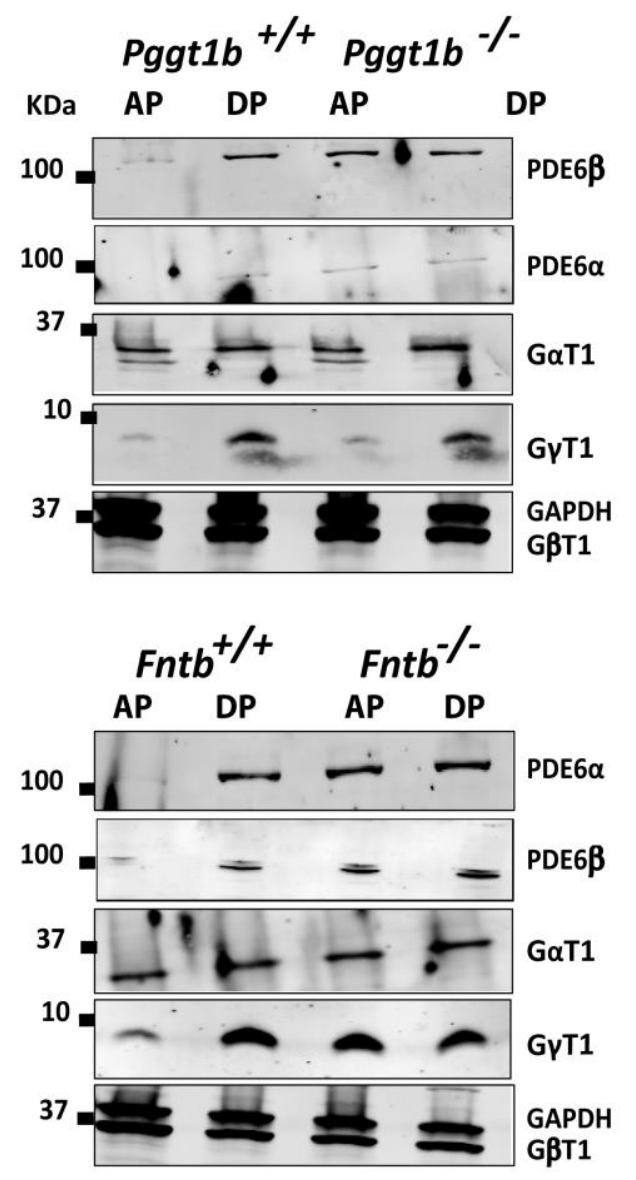

B
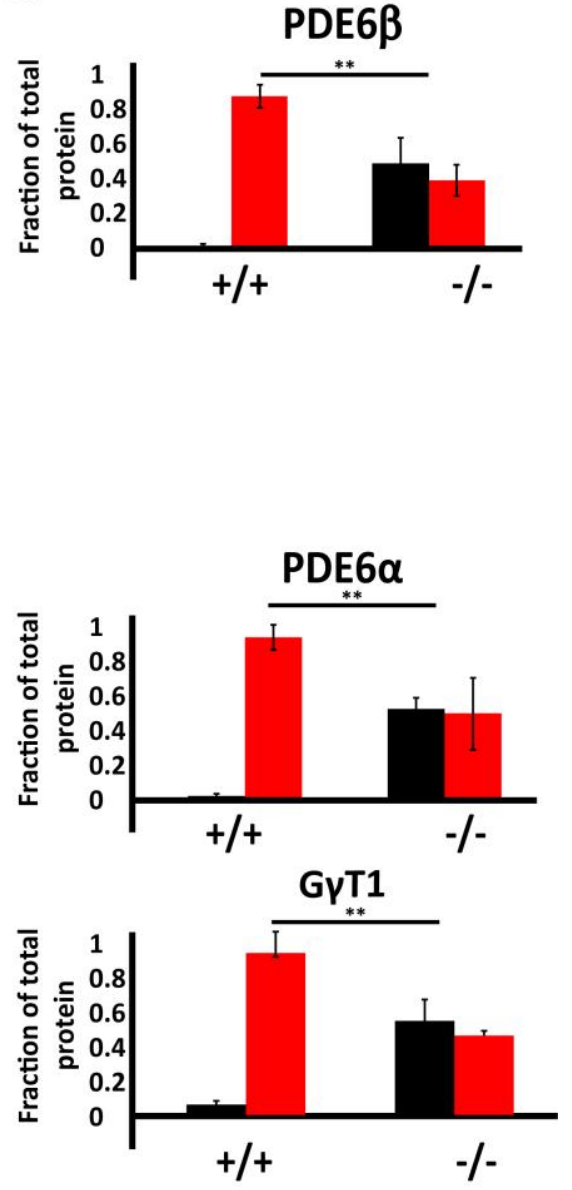

Figure. 6 Defective partitioning of phosphodiesterase and transducin- $\gamma$ subunit in Triton $X$ 114 detergent in the absence of prenylation. 
Figure 7. Transducin is mislocalized in the IS in the absence of farnesylation

(A-C) Immunofluorescence labeling of P60 Fntb ${ }^{+/+}$and $\mathrm{Fntb}^{-/-}$mice littermates. Rod

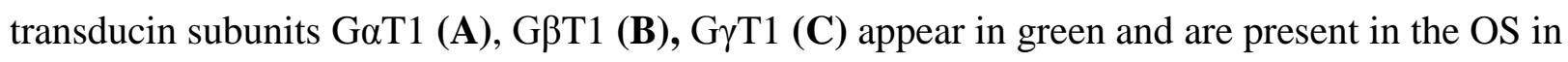
$\mathrm{Fntb}^{+/+}$but are mislocalized partially to IS in $\mathrm{Fntb}{ }^{-/-}$mice (magnified images and arrowheads), CNGA1/3 in red labels the OS, nuclei are stained with DAPI (blue). (D-F)

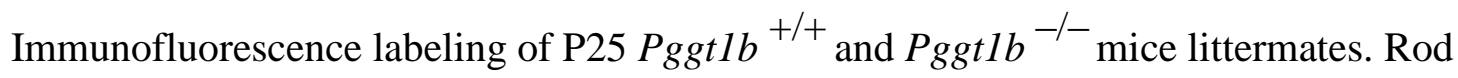

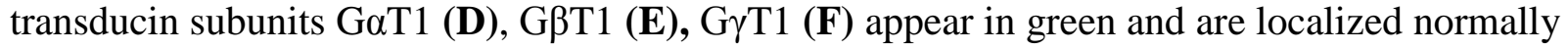
to OS in both $P_{\text {ggtl }}{ }^{+/+}$and Pggtl $b^{-/-}$mice. Scale $=10 \mu \mathrm{M}$ 

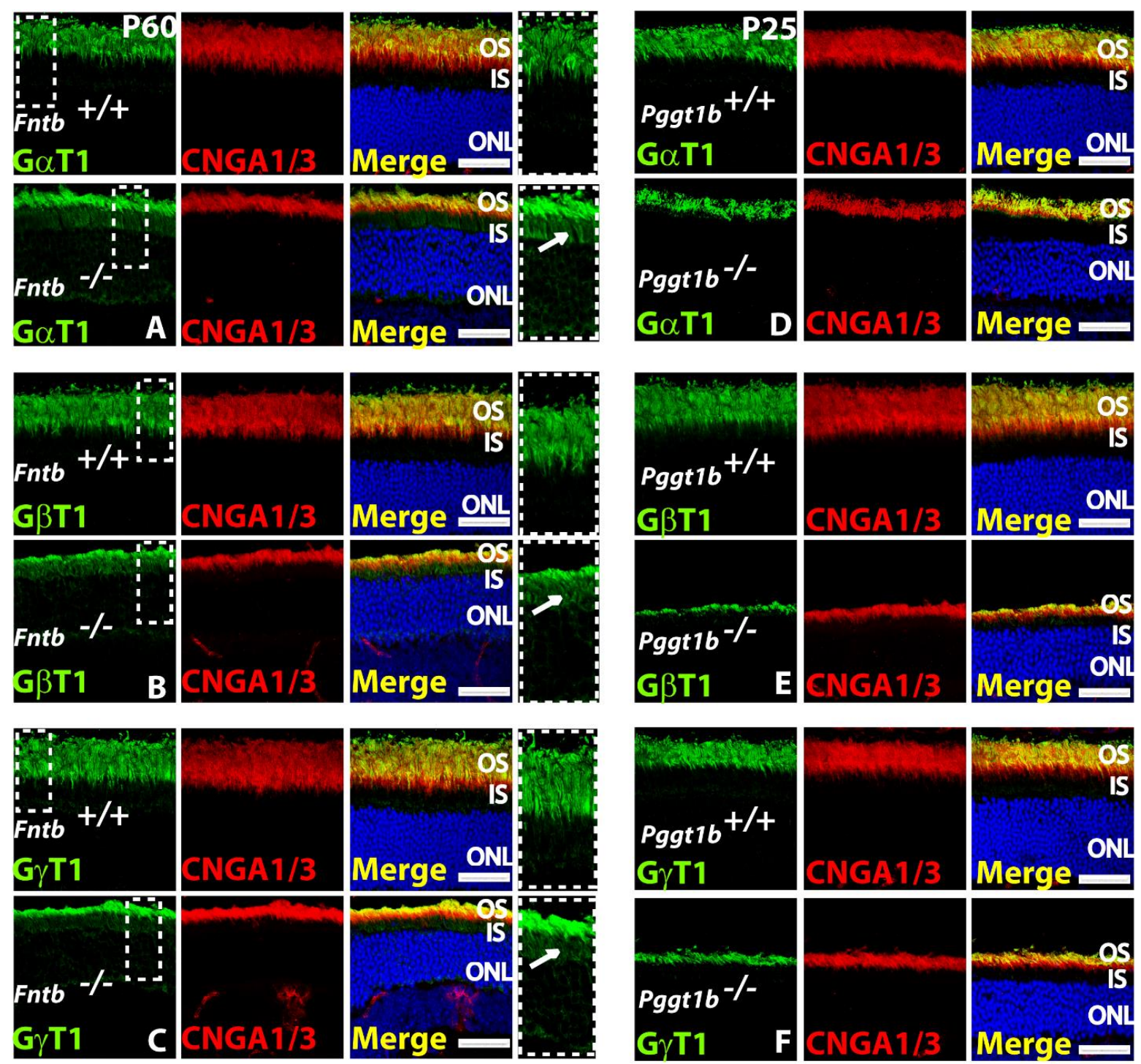

Figure. 7 Transducin is mislocalized in the IS in the absence of farnesylation 
Figure 8 Lack of Fntb results in slow reappearance of translocated transducin to rod outer segments

(I) Immunohistochemical localization of P18 Fntb ${ }^{+/+}$and $\mathrm{Fntb}^{-/-}$mice littermates. The sections were prepared from fully dark adapted retinas of $F n t b^{+/+}$and $F n t b^{-/-}$mice littermates (A), complete light saturation with light intensity of 1500 lux (B), dark adaptation for $2 \mathrm{~h}, 12 \mathrm{~h}$ and 24h after light saturation (C, D and E respectively). (II and III) Transducin G $\gamma \mathrm{T} 1$ content in the outer segment and inner segment before and after light saturation. The immunoreactivities of G $\gamma \mathrm{T} 1$ detected in OS and IS regions were quantified by ImageJ software. The signal intensities of $\mathrm{G} \gamma \mathrm{T} 1$ in $\mathrm{OS}$ were expressed as percentage of total intensities of $\mathrm{G} \gamma \mathrm{T} 1$ in photoreceptor layers. All calculations were expressed as mean SD of three regions of different sections. (F-H) Immunohistochemical localization of $\mathrm{Pggt} \mathrm{b}^{+/+}$and $P g g t 1 b^{-/-}$mice littermates at P18. The sections were prepared from fully dark adapted retinas of Pggtl $^{+/+}$and $P g g t 1 b^{-/-}$mice littermates (F), complete light saturation with light intensity of 1500 lux (G), dark adaptation for 2h after light saturation $(\mathrm{H}),\left(n=3 . P<0.002\right.$, black bar, G $\gamma \mathrm{T} 1 F_{n t b}{ }^{+/+}$; red bar, G $\gamma \mathrm{T} 1$ in $F n t b^{-}$ I-) 
A B

C
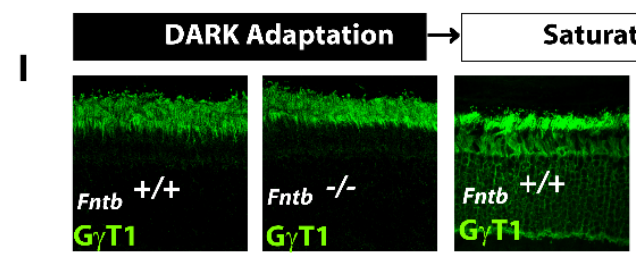

Bing Light
Fntb $-1-$
G $\gamma$ T1

2h DARK
Fntb ${ }^{+/+}$
G $\gamma 1$

\begin{tabular}{l} 
Adaptation \\
\hline \\
\hline Entb -1 \\
G $\gamma \mathrm{T} 1$
\end{tabular}

II
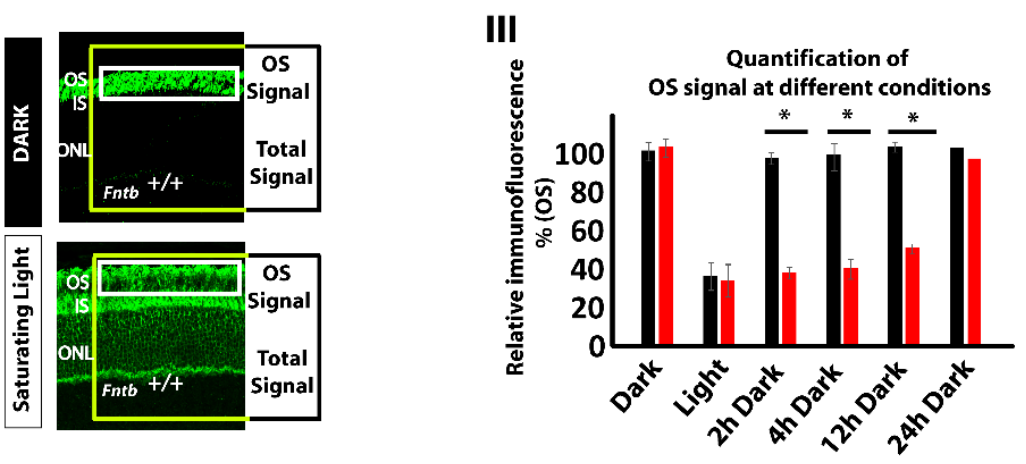

H

IV

$\mathbf{F}$

G
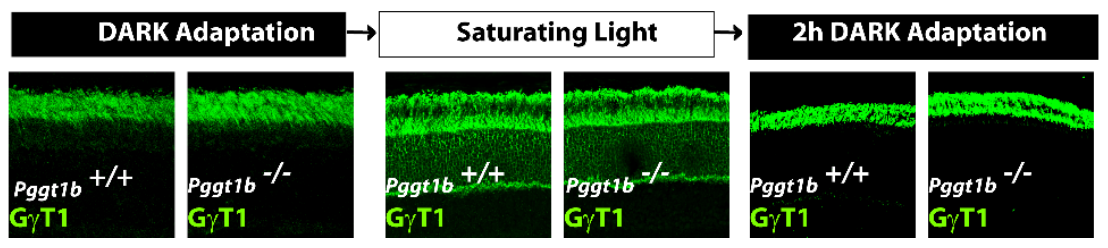

Figure. 8 Lack of Fntb affects the reappearance of translocated transducin to rod outer segments 
Figure 9 Levels of rod PDE6 and GRK-1 are severely affected in the absence of Pggt1b or Fntb

(A \& C) Immunofluorescent labeling of P25 Pggt1 ${ }^{+/+}$and Pggtl $^{-/-}$littermates.

Immunofluorescent labeling of retinae from P25 Pggtl $^{+/+}$and Pggtl $^{-/-}$littermates. (A)

CNGA1/3 (red) labels the OS; MOE antibody staining rod and cone PDE6 is in green. (C)

CNGA1/3 (red); GRK1 in green, Nuclei are stained with DAPI in blue. Note that GRK1 is

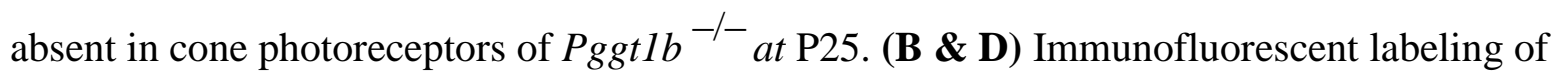
retinae from P45 Fntb ${ }^{+/+}$and $F n t b^{-/-}$littermates. (B) CNGA1/3 (red) labels the OS; MOE antibody staining in green. (D) CNGA1/3 (red) co-localized with GRK1 is shown in green. Scale $=10 \mu \mathrm{M}$. 

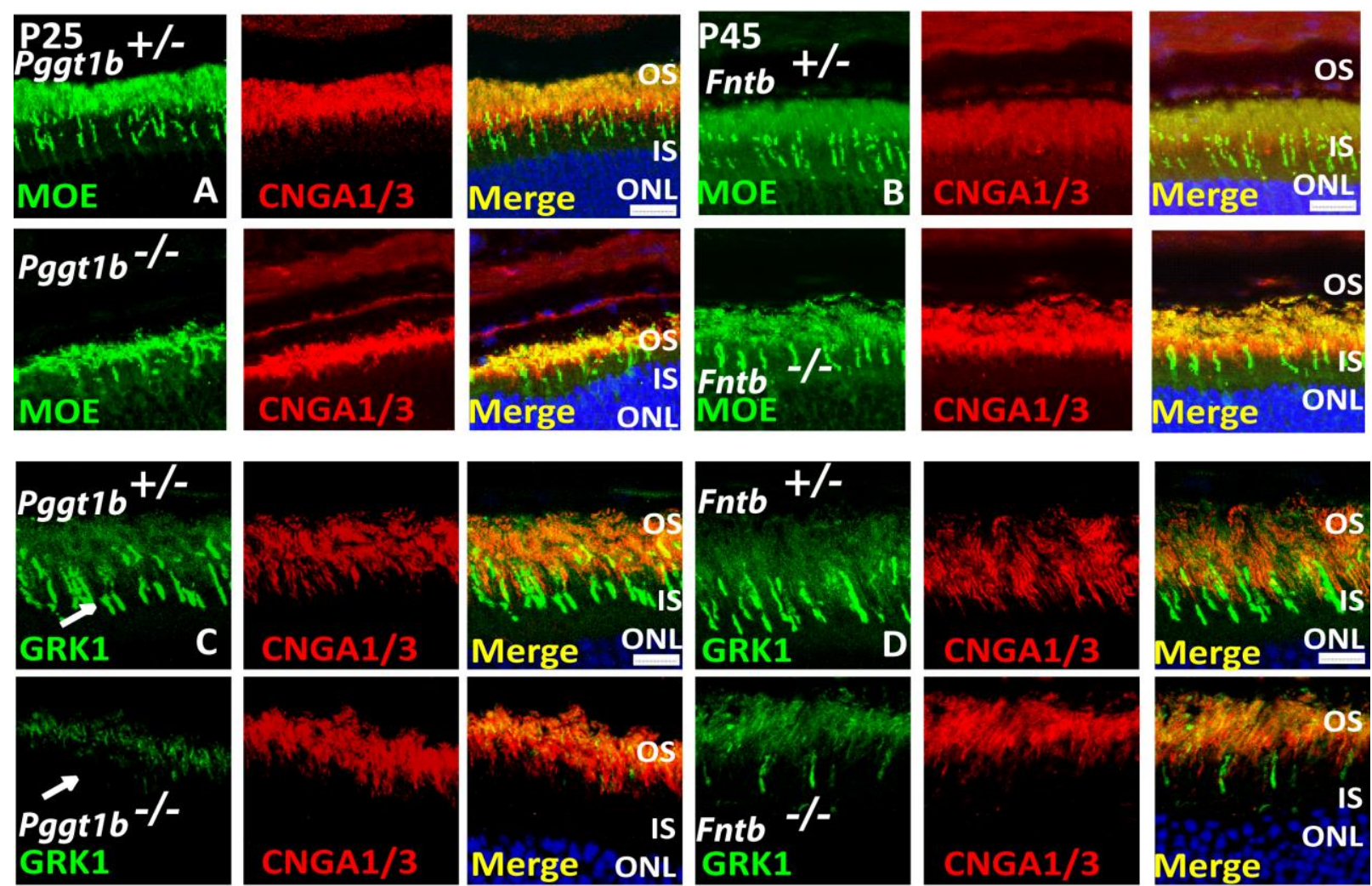

Figure. 9 Levels of rod PDE6 and GRK-1 are severely affected in the absence of Pggtlb or Fntb 
Figure 10. Rod PDE6 assembly is not affected in the photoreceptors lacking Pggtlb and Fntb

Immunoprecipitation (IP) of rod PDE6 subunit from the retinal extracts of Pggtlb and Fntb mice littermates at P16, using monoclonal ROS-1 antibody. After ROS-1 IP, immunoblots were probed with antibodies against catalytic subunits of rod PDE6. Control IP with non-specific mouse IgG is shown. Cryo-section of Pggtlb and Fntb deficient mice stained using ROS-1 antibody $(n=3 ; * * P<0.05, \mathrm{~T}=$ total fraction; $\mathrm{U}=$ Unbound fraction; $\mathrm{B}=$ Bound fraction $)$. 


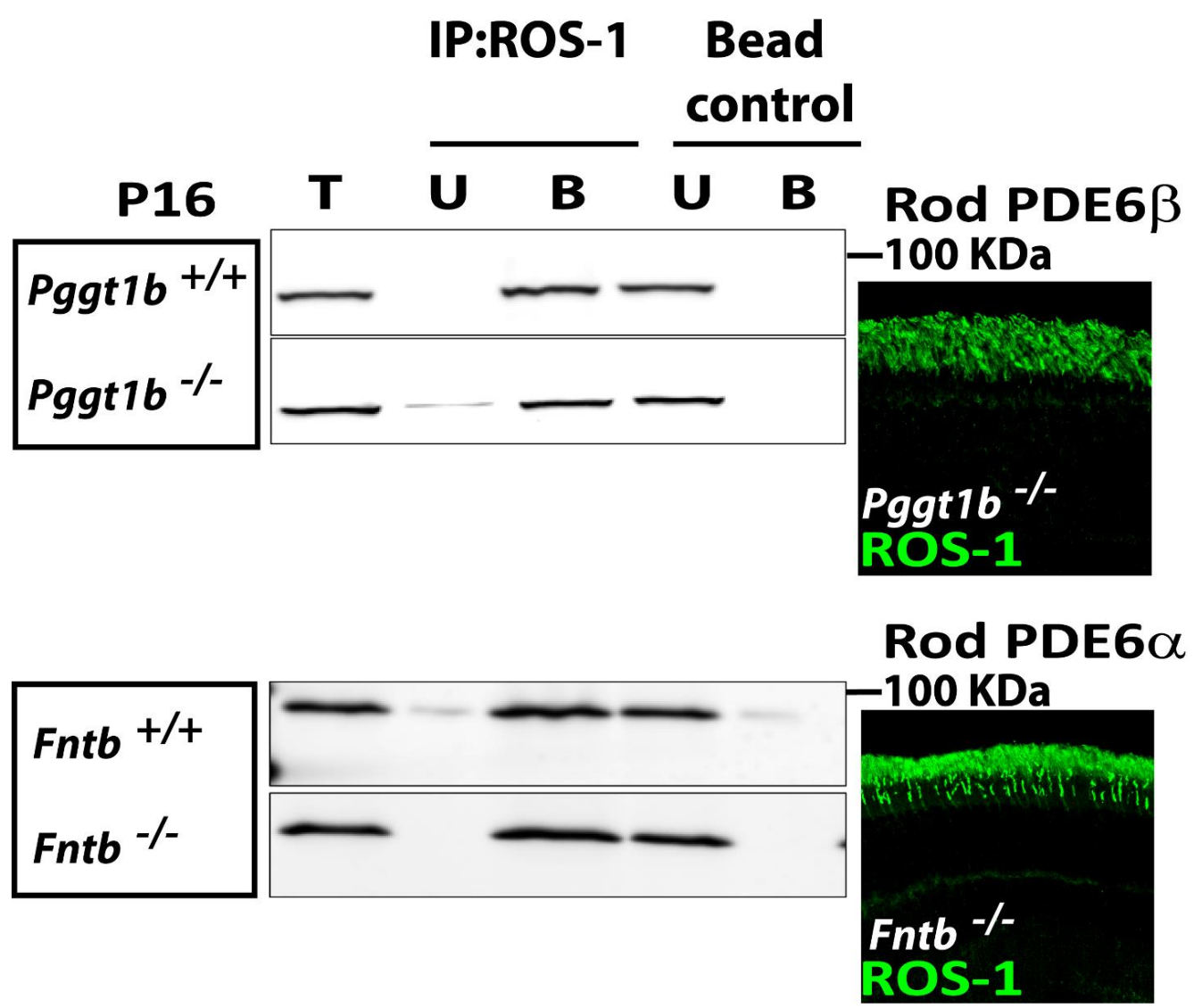

Figure. 10 Rod PDE6 assembly is not affected in the photoreceptors lacking Pggt1b and Fntb 


\section{REFERENCES}

1 Casey, P.J. and Seabra, M.C. (1996) Protein prenyltransferases. Journal of Biological Chemistry, 271, 5289-5292.

2 Cox, A.D. and Der, C.J. (1992) Protein prenylation: more than just glue? Current opinion in cell biology, 4, 1008-1016.

3 Zhang, F.L. and Casey, P.J. (1996) Protein prenylation: molecular mechanisms and functional consequences. Annual review of biochemistry, 65, 241-269.

4 Apolloni, A., Prior, I.A., Lindsay, M., Parton, R.G. and Hancock, J.F. (2000) H-ras but not K-ras traffics to the plasma membrane through the exocytic pathway. Molecular and cellular biology, 20, 2475-2487.

5 Choy, E., Chiu, V.K., Silletti, J., Feoktistov, M., Morimoto, T., Michaelson, D., Ivanov, I.E. and Philips, M.R. (1999) Endomembrane trafficking of ras: the CAAX motif targets proteins to the ER and Golgi. Cell, 98, 69-80.

6 Seabra, M.C. (1998) Membrane association and targeting of prenylated Ras-like GTPases. Cellular signalling, 10, 167-172.

$7 \quad$ Mijimolle, N., Velasco, J., Dubus, P., Guerra, C., Weinbaum, C.A., Casey, P.J., Campuzano, V. and Barbacid, M. (2005) Protein farnesyltransferase in embryogenesis, adult homeostasis, and tumor development. Cancer cell, 7, 313-324.

8 Lee, R., Chang, S.Y., Trinh, H., Tu, Y., White, A.C., Davies, B.S., Bergo, M.O., Fong, L.G., Lowry, W.E. and Young, S.G. (2010) Genetic studies on the functional relevance of the protein prenyltransferases in skin keratinocytes. Human molecular genetics, in press., ddq036. 9 Khan, O.M., Ibrahim, M.X., Jonsson, M., Karlsson, C., Liu, M., Sjogren, A.-K.M., Olofsson, F.J., Brisslert, M., Andersson, S. and Ohlsson, C. (2011) Geranylgeranyltransferase type I (GGTase-I) deficiency hyperactivates macrophages and induces erosive arthritis in mice. The Journal of clinical investigation, 121, 628-639.

10 Hrycyna, C., Bergo, M.O. and Tamanoi, F. (2011) Protein Prenylation. Academic Press.

11 Beigneux, A., Withycombe, S.K., Digits, J.A., Tschantz, W.R., Weinbaum, C.A., Griffey, S.M., Bergo, M., Casey, P.J. and Young, S.G. (2002) Prenylcysteine lyase deficiency in mice results in the accumulation of farnesylcysteine and geranylgeranylcysteine in brain and liver. Journal of Biological Chemistry, 277, 38358-38363.

12 Yang, S.H., Bergo, M.O., Farber, E., Qiao, X., Fong, L.G. and Young, S.G. (2009) Caution! Analyze transcripts from conditional knockout alleles. Transgenic research, 18, 483489.

13 Yang, S.H., Meta, M., Qiao, X., Frost, D., Bauch, J., Coffinier, C., Majumdar, S., Bergo, M.O., Young, S.G. and Fong, L.G. (2006) A farnesyltransferase inhibitor improves disease phenotypes in mice with a Hutchinson-Gilford progeria syndrome mutation. The Journal of clinical investigation, 116, 2115-2121.

14 Anant, J., Ong, O., Xie, H., Clarke, S., O'Brien, P. and Fung, B. (1992) In vivo differential prenylation of retinal cyclic GMP phosphodiesterase catalytic subunits. Journal of Biological Chemistry, 267, 687-690. 
15 Ong, O.C., Ota, I.M., Clarke, S. and Fung, B. (1989) The membrane binding domain of rod cGMP phosphodiesterase is posttranslationally modified by methyl esterification at a Cterminal cysteine. Proceedings of the National Academy of Sciences, 86, 9238-9242.

16 Fukada, Y., Takao, T., Ohguro, H., Yoshizawa, T., Akino, T. and Shimonishi, Y. (1990) Farnesylated gamma-subunit of photoreceptor $\mathrm{G}$ protein indispensable for GTP-binding. Nature, 346, 658-660.

17 Matsuda, T., Takao, T., Shimonishi, Y., Murata, M., Asano, T., Yoshizawa, T. and Fukada, Y. (1994) Characterization of interactions between transducin alpha/beta gammasubunits and lipid membranes. Journal of Biological Chemistry, 269, 30358-30363.

18 Baehr, W., Devlin, M.J. and Applebury, M.L. (1979) Isolation and characterization of cGMP phosphodiesterase from bovine rod outer segments. J Biol Chem, 254, 11669-11677.

19 Inglese, J., Koch, W.J., Caron, M.G. and Lefkowitz, R.J. (1992) Isoprenylation in regulation of signal transduction by G-protein-coupled receptor kinases. Nature, 359, 147-150.

20 Burns, M.E. and Arshavsky, V.Y. (2005) Beyond counting photons: trials and trends in vertebrate visual transduction. Neuron, 48, 387-401.

21 Goc, A., Chami, M., Lodowski, D.T., Bosshart, P., Moiseenkova-Bell, V., Baehr, W., Engel, A. and Palczewski, K. (2010) Structural characterization of the rod cGMP phosphodiesterase 6. Journal of molecular biology, 401, 363-373.

22 Angleson, J.K. and Wensel, T.G. (1993) A GTPase-accelerating factor for transducin, distinct from its effector cGMP phosphodiesterase, in rod outer segment membranes. Neuron, 11, 939-949.

23 Wensel, T.G. (2008) Signal transducing membrane complexes of photoreceptor outer segments. Vision research, 48, 2052-2061.

24 Wensel, T.G. and Stryer, L. (1986) Reciprocal control of retinal rod cyclic GMP phosphodiesterase by its $\gamma$ subunit and transducin. Proteins: Structure, Function, and Bioinformatics, 1, 90-99.

25 Artemyev, N.O., Natochin, M., Busman, M., Schey, K.L. and Hamm, H.E. (1996) Mechanism of photoreceptor cGMP phosphodiesterase inhibition by its gamma-subunits. Proceedings of the National Academy of Sciences, 93, 5407-5412.

26 Zhang, Z., He, F., Constantine, R., Baker, M.L., Baehr, W., Schmid, M.F., Wensel, T.G. and Agosto, M.A. (2015) Domain organization and conformational plasticity of the G protein effector, PDE6. Journal of Biological Chemistry, 290, 12833-12843.

27 Kolandaivelu, S., Huang, J., Hurley, J.B. and Ramamurthy, V. (2009) AIPL1, a protein associated with childhood blindness, interacts with $\alpha$-subunit of rod phosphodiesterase (PDE6) and is essential for its proper assembly. Journal of Biological Chemistry, 284, 30853-30861.

28 Kolandaivelu, S. and Ramamurthy, V. (2014), In Retinal Degenerative Diseases. Springer, in press., pp. 43-48.

29 Kolandaivelu, S., Singh, R.K. and Ramamurthy, V. (2013) AIPL1, A protein linked to blindness, is essential for the stability of enzymes mediating cGMP metabolism in cone photoreceptor cells. Human molecular genetics, in press., ddt496.

30 Kokame, K., Fukada, Y., Yoshizawa, T., Takao, T. and Shimonishi, Y. (1992) Lipid modification at the $\mathrm{N}$ terminus of photoreceptor G-protein $\alpha$-subunit. in press.

31 Linder, M.E., Middleton, P., Hepler, J.R., Taussig, R., Gilman, A.G. and Mumby, S.M. (1993) Lipid modifications of G proteins: alpha subunits are palmitoylated. Proceedings of the National Academy of Sciences, 90, 3675-3679. 
32 Wedegaertner, P.B., Wilson, P.T. and Bourne, H.R. (1995) Lipid modifications of trimeric G proteins. Journal of Biological Chemistry, 270, 503-506.

33 Christiansen, J.R., Pendse, N.D., Kolandaivelu, S., Bergo, M.O., Young, S.G. and Ramamurthy, V. (2016) Deficiency of Isoprenylcysteine Carboxyl Methyltransferase (ICMT) Leads to Progressive Loss of Photoreceptor Function. The Journal of Neuroscience, 36, 51075114.

34 Lobanova, E.S., Finkelstein, S., Song, H., Tsang, S.H., Chen, C.-K., Sokolov, M., Skiba, N.P. and Arshavsky, V.Y. (2007) Transducin translocation in rods is triggered by saturation of the GTPase-activating complex. The Journal of neuroscience, 27, 1151-1160.

35 Sokolov, M., Lyubarsky, A.L., Strissel, K.J., Savchenko, A.B., Govardovskii, V.I., Pugh, E.N. and Arshavsky, V.Y. (2002) Massive light-driven translocation of transducin between the two major compartments of rod cells: a novel mechanism of light adaptation. Neuron, 34, 95106.

36 Sokolov, M., Strissel, K.J., Leskov, I.B., Michaud, N.A., Govardovskii, V.I. and Arshavsky, V.Y. (2004) Phosducin Facilitates Light-driven Transducin Translocation in Rod Photoreceptors EVIDENCE FROM THE PHOSDUCIN KNOCKOUT MOUSE. Journal of Biological Chemistry, 279, 19149-19156.

37 Calvert, P.D., Strissel, K.J., Schiesser, W.E., Pugh, E.N. and Arshavsky, V.Y. (2006) Light-driven translocation of signaling proteins in vertebrate photoreceptors. Trends in cell biology, 16, 560-568.

38 Kassai, H., Aiba, A., Nakao, K., Nakamura, K., Katsuki, M., Xiong, W.-H., Yau, K.-W., Imai, H., Shichida, Y. and Satomi, Y. (2005) Farnesylation of retinal transducin underlies its translocation during light adaptation. Neuron, 47, 529-539.

39 Pittler, S.J., Fliesler, S.J., Fisher, P.L., Keller, P. and Rapp, L.M. (1995) In vivo requirement of protein prenylation for maintenance of retinal cytoarchitecture and photoreceptor structure. The Journal of cell biology, 130, 431-439.

40 Furuta, Y., Lagutin, O., Hogan, B.L. and Oliver, G.C. (2000) Retina- and ventral forebrain- specific Cre recombinase activity in transgenic mice. Genesis, 26, 130-132.

41 Liu, M., Sjogren, A.-K.M., Karlsson, C., Ibrahim, M.X., Andersson, K.M., Olofsson, F.J., Wahlstrom, A.M., Dalin, M., Yu, H. and Chen, Z. (2010) Targeting the protein prenyltransferases efficiently reduces tumor development in mice with K-RAS-induced lung cancer. Proceedings of the National Academy of Sciences, 107, 6471-6476.

42 Sjogren, A.-K.M., Andersson, K.M., Liu, M., Cutts, B.A., Karlsson, C., Wahlstrom, A.M., Dalin, M., Weinbaum, C., Casey, P.J. and Tarkowski, A. (2007) GGTase-I deficiency reduces tumor formation and improves survival in mice with K-RAS-induced lung cancer. The Journal of clinical investigation, 117, 1294-1304.

43 Christiansen, J.R., Kolandaivelu, S., Bergo, M.O. and Ramamurthy, V. (2011) RASconverting enzyme 1-mediated endoproteolysis is required for trafficking of rod phosphodiesterase 6 to photoreceptor outer segments. Proceedings of the National Academy of Sciences, 108, 8862-8866.

44 Kolandaivelu, S., Chang, B. and Ramamurthy, V. (2011) Rod phosphodiesterase-6 (PDE6) catalytic subunits restore cone function in a mouse model lacking cone PDE6 catalytic subunit. Journal of Biological Chemistry, 286, 33252-33259.

45 Justice, J.M., Murtagh, J.J., Moss, J. and Vaughan, M. (1995) Hydrophobicity and subunit interactions of rod outer segment proteins investigated using Triton X-114 phase partitioning. Journal of Biological Chemistry, 270, 17970-17976. 
46 Roosing, S., Rohrschneider, K., Beryozkin, A., Sharon, D., Weisschuh, N., Staller, J., Kohl, S., Zelinger, L., Peters, T.A. and Neveling, K. (2013) Mutations in RAB28, encoding a farnesylated small GTPase, are associated with autosomal-recessive cone-rod dystrophy. The American Journal of Human Genetics, 93, 110-117.

47 Hurwitz, R.L., Bunt-Milam, A.H. and Beavo, J.A. (1984) Immunologic characterization of the photoreceptor outer segment cyclic GMP phosphodiesterase. Journal of Biological Chemistry, 259, 8612-8618.

48 Rattner, A., Sun, H. and Nathans, J. (1999) Molecular genetics of human retinal disease. Annual review of genetics, 33, 89-131.

49 Hartong, D.T., Berson, E.L. and Dryja, T.P. (2006) Retinitis pigmentosa. The Lancet, 368, 1795-1809.

50 Saada, J., Perrault, I., Amiel, J., Litzler, J., Filhol, E., Elkhartoufi, N., Kwong, M., Burglen, L., Chassaing, N. and é Encha-Ravazi, F. A Homozygous PDE6D Mutation in Joubert Syndrome Impairs Targeting of Farnesylated INPP5E Protein to the Primary Cilium. in press. 51 Bielas, S.L., Silhavy, J.L., Brancati, F., Kisseleva, M.V., Al-Gazali, L., Sztriha, L., Bayoumi, R.A., Zaki, M.S., Abdel-Aleem, A. and Rosti, R.O. (2009) Mutations in INPP5E, encoding inositol polyphosphate-5-phosphatase E, link phosphatidyl inositol signaling to the ciliopathies. Nature genetics, 41, 1032-1036.

52 Jacoby, M., Cox, J.J., Gayral, S., Hampshire, D.J., Ayub, M., Blockmans, M., Pernot, E., Kisseleva, M.V., Compère, P. and Schiffmann, S.N. (2009) INPP5E mutations cause primary cilium signaling defects, ciliary instability and ciliopathies in human and mouse. Nature genetics, 41, 1027-1031.

53 Rao, K.N., Zhang, W., Li, L., Anand, M. and Khanna, H. (2016) Prenylated retinal ciliopathy protein RPGR interacts with PDE6 $\delta$ and regulates ciliary localization of Joubert syndrome-associated protein INPP5E. Human Molecular Genetics, in press., ddw281.

54 Baehr, W. (2014) Membrane Protein Transport in Photoreceptors: The Function of PDE $\delta$ The Proctor Lecture. Investigative ophthalmology \& visual science, 55, 8653-8666.

55 Zhang, H., Li, S., Doan, T., Rieke, F., Detwiler, P., Frederick, J. and Baehr, W. (2007) Deletion of $\mathrm{PrBP} / \delta$ impedes transport of GRK1 and PDE6 catalytic subunits to photoreceptor outer segments. Proceedings of the National Academy of Sciences, 104, 8857-8862.

56 Constantine, R., Zhang, H., Gerstner, C.D., Frederick, J.M. and Baehr, W. (2012) Uncoordinated (UNC) 119: coordinating the trafficking of myristoylated proteins. Vision research, 75, 26-32.

57 Zhang, H., Constantine, R., Vorobiev, S., Chen, Y., Seetharaman, J., Huang, Y.J., Xiao, R., Montelione, G.T., Gerstner, C.D. and Davis, M.W. (2011) UNC119 is required for G protein trafficking in sensory neurons. Nature neuroscience, 14, 874-880.

58 Finkelstein, S., Lobanova, E. and Arshavsky, V. (2009) Knockout of the Rod Transducin Gamma Subunit Induces Cell Death. Investigative Ophthalmology \& Visual Science, 50, 44724472.

59 Kirschman, L.T., Kolandaivelu, S., Frederick, J.M., Dang, L., Goldberg, A.F., Baehr, W. and Ramamurthy, V. (2009) The Leber congenital amaurosis protein, AIPL1, is needed for the viability and functioning of cone photoreceptor cells. Human molecular genetics, in press., ddp571. 


\section{CHAPTER 4}

Deficiency of isoprenylcysteine carboxyl methyltransferase (ICMT) leads to progressive loss of photoreceptor function

(J Neurosci. 2016 May 4;36(18):5107-14.)

Jeffrey R. Christiansen ${ }^{*}$, Nachiket D. Pendse $1,2,5$ *, Saravanan Kolandaivelu 1 , Martin

O. Bergo ${ }^{3}$, Stephen G. Young 4 , and Visvanathan Ramamurthy $1,2,5 * *$

Departments of Ophthalmology 1 , Biology ${ }^{2}$, and Biochemistry 5 , West Virginia University;

${ }^{3}$ Sahlgrenska Cancer Center, University of Gothenburg, Sweden; ${ }^{4}$ Departments of Medicine and Human Genetics, University of California, Los Angeles

Author contributions: J.R.C., N.D.P., S.K., and V.R. designed research; J.R.C., N.D.P., S.K. performed research; J.R.C., N.D.P. and V.R. analyzed data; M.O.B. and S.Y. provided advice and reagents, helped to prepare the manuscript; J.R.C., N.D.P. and V.R. wrote the manuscript. This work was supported by National Institutes of Health Grants R01EY017035 (to V.R.) and R01HL126551 (to S.Y.), West Virginia Lions, and an Unrestricted Research to Prevent Blindness Challenge Grant (West Virginia University). We thank Thamarai Saravanan for maintaining mice and Dr. Karen Martin for advice and the use of the West Virginia University Microscopic Imaging Facility. We also thank the members of the Ramamurthy laboratory for constructive criticisms.

* J.R.C. and N.D.P. contributed equally to this work.

**Correspondence should be addressed to Visvanathan Ramamurthy, Department of Ophthalmology, One Medical Center Drive, West Virginia University, Morgantown, WV 265069193 USA; ramamurthyv@wvumedicine.org 


\section{ABSTRACT}

Retinal neurons use multiple strategies to fine-tune visual signal transduction, including posttranslational modifications of proteins such as addition of an isoprenyl lipid to a carboxylterminal cysteine in proteins that terminate with "CAAX motif." We previously showed that RAS converting enzyme 1 (RCE1) - mediated processing of isoprenylated proteins is required for photoreceptor maintenance and function. However, it is not yet known whether the requirement for the RCE1-mediated protein processing is related to the absence of the endoproteolytic processing step, the absence of the subsequent methylation step by isoprenylcysteine methyltransferase (ICMT), or both. To approach this issue and to understand the significance of protein methylation, we generated mice lacking Icmt expression the retina. In the absence of Icmt expression, rod and cone light-mediated responses diminished progressively. Lack of ICMTmediated methylation led to defective association of isoprenylated transducin and cone phosphodiesterase $6\left(\mathrm{PDE} 6 \alpha^{\prime}\right)$ with photoreceptor membranes and resulted in decreased levels of transducin, PDE6 $\alpha^{\prime}$, and cone G-protein receptor kinase -1 (GRK1). In contrast to our earlier findings with retina-specific Rcel knockout mice, rod PDE6 in Icmt-deficient mice trafficked normally to the photoreceptor outer segment (OS), suggesting that the failure to remove the $A A X$ is responsible for blocking the movement of PDE6 to the OS. Our findings demonstrate that carboxyl methylation of isoprenylated proteins is crucial for maintenance of photoreceptor function. 


\section{SIGNIFICANCE STATEMENT}

In this report, we show that an absence of ICMT-mediated protein methylation leads to progressive loss of vision. Photoreceptors also degenerate, although at a slower pace than the rate of visual loss. The reduction in photoresponses is due to defective association of crucial players in phototransduction cascade. Unlike the situation with RCE1 deficiency, where both methylation and removal of $-A A X$ was affected, the transport of isoprenylated proteins in ICMTdeficient retinas was not dependent on methylation. This finding implies that the retention of the $-A A X$ in PDE6 catalytic subunits in $R c e 1^{-/-}$mice is responsible for impeding their transport to the rod photoreceptor outer segment. In conclusion, lack of methylation of isoprenylcysteines leads to age-dependent photoreceptor dysfunction. 


\section{INTRODUCTION}

Prenylation refers to the addition of a 15-carbon farnesyl or a 20-carbon geranylgeranyl lipid to the C-terminal cysteine of proteins ending in a " $C A A X$ " motif, where $C$ refers to a cysteine, $A$ for an aliphatic amino acid, and $X$ for any amino acid (Svensson, Casey et. al. 2006)

(Fig. 1, step 1). After prenylation, the last three amino acid residues $(-A A X)$ are cleaved by RASconverting enzyme 1 (RCE1) at the endoplasmic reticulum (ER) membrane (Fig. 1, step 2). The final event of "CAAX protein" processing is methyl esterification of the newly exposed isoprenyl cysteine by ICMT, an integral ER membrane protein that uses $S$-adenosyl methionine (SAM) as the methyl donor (Fig. 1, Step 3) (47) (48).

Experiments designed to identify methylated photoreceptor proteins uncovered a subset of rod outer segment proteins that incorporated a radioactive methyl group (50). The identity of the methylated proteins and their ability to incorporate a methyl group has been studied extensively, but the importance of this modification to photoreceptor function is not known $(37,39,51)$. Rod phosphodiesterase 6 (PDE6), the effector enzyme of the visual signal transduction cascade, was the first methylated protein to be identified in retinal lysates (50). The carboxyl terminus of PDE6 catalytic subunits terminates with a so-called "CAAX motif," which triggers isoprenylation of the carboxyl-terminal cysteine (the " $C$ " of the $C A A X$ motif). PDE6 $\alpha$ and $\beta$ catalytic subunits are isoprenylated by farnesyl and geranylgeranyl lipids, respectively. Interestingly, rod PDE6 $\alpha$ incorporated a methyl group in an in vitro radioactive methylation assay, whereas PDE6 $\beta$ was not an efficient substrate for protein methylation (38). The methylation status of PDE6 $\alpha^{\prime}$, which is thought to be geranylgeranylated, is not known. Additional isoprenylated (farnesylated) photoreceptor proteins include the $\gamma$-subunit of transducin (G $\gamma \mathrm{T} 1)$ and rhodopsin kinase (GRK1) (37,52). 
Methylation of isoprenylated cysteines is thought to increase the hydrophobicity of the protein and facilitate interactions with membranes (56). Also, in vitro approaches have shown that carboxyl methylation enhances certain protein-protein interactions. For example, methylation of $\mathrm{G} \gamma \mathrm{T} 1$ is thought to enhance the interaction of the transducin complex with metarhodopsin II (51). Furthermore, the interaction between C-terminal isoprenylated PDE6 peptides and prenyl binding protein $\delta(\operatorname{PrBP} \delta)$ is influenced by the methylation status of PDE6 (57). Methylation has been proposed to be a dynamic modification but conclusive evidence for reversibility of this modification is still lacking. Early studies suggest that methylation of G $\gamma \mathrm{T} 1$ is reversible, but experiments purifying transducin from photoreceptors failed to identify a nonmethylated isoform (Fukada et al. 1994, Perez et.al 1991).

A requirement for $C A A X$ protein processing in retinal function was demonstrated by a study in which Rcel was inactivated in the neural retina (Christiansen et al. 2011). An absence of RCE1-mediated protein processing disrupted the transport of PDE6 to the outer segment (OS). That study did not determine whether the defective PDE6 transport in retinal neurons was due to lack of RCE1-mediated processing, the absence of the subsequent methylation step, or to the absence of both processing steps.

The importance of ICMT-mediated methyl esterification has been demonstrated with gene-targeted mice where it was observed that inactivation of Icmt caused death during embryonic development (Bergo et al. 2001, Kim et al. 1999). Therefore, to specifically investigate the role of methyl esterification on retinal proteins, we eliminated Icmt expression in the retina with $C r e-l o x P$ recombination techniques. The retina-specific Icmt knockout mice were created by breeding $I c m t / f l$ mice with mice expressing $C r e$ recombinase under the control of the retina- and forebrain-specific promoter Six3 $(55,155)$. In this study, we combined histology, 
electroretinography, and biochemistry to assess the functional relevance of ICMT-mediated protein processing on the function of the photoreceptor neurons. 


\section{MATERIALS AND METHODS}

\section{Mouse models}

Mice homozygous for conditional Icmt knockout alleles $\left(\mathrm{Icmt} \mathrm{fl}^{\mathrm{fl}}\right.$ ) were bred with mice hemizygous for Six3-Cre transgene to create Icmtfl/flSix3-Cre mice, which have a retina-specific inactivation of Icmt $(55,155)$. Littermate $I c m t^{w t} / f l$ Six3-Cre mice were used as controls. Both males and females were used in the study. Genotyping was performed by PCR amplification using genomic DNA extracted from mouse ear punch biopsies as described earlier (Court et al. 2013). Mouse experiments were performed in accordance with the National Institutes of Health guidelines and the protocol approved by Institutional Animal Care and Use Committee of West Virginia University.

\section{Quantitative PCR}

Retinas were dissected from freshly enucleated eyes and frozen on dry ice in the presence of Trizol (Invitrogen). RNA extracted from frozen retinas was used to generate cDNA with Qscript (Quanta Bioscience). $300 \mu \mathrm{g}$ of cDNA from heterozygous and knockout littermates (in triplicate) was used as a template for Quantitative PCR using MyiQ PCR cycler (Bio-Rad) and MyiQ SYBR Green Supermix (Bio-Rad). Icmt was amplified with primers 5'CGCCTCAGCCTCGCTACATT-3' (exon 1) and 5' - TTGGAGCCAGCCGTAAACAT-3' (exon 4), yielding a 509-base pair (bp) product. Threshold values were normalized to hypoxanthine phosphoribosyltransferase (Hprt) gene expression levels with primers 5'CAAACTTTGCTTTCCCTGGT-3' and 5'-CAAGGGCATATCCAACAACA-3' (250-bp product). 
Electroretinography $(E R G)$

Littermates were dark-adapted overnight, and the eyes were dilated (1:1 phenylephrine: tropicamide) for $10 \mathrm{~min}$. Isoflurane anesthesia ( $1.5 \%$ in $2.5 \%$ oxygen) was administered via nose cone on a $37^{\circ} \mathrm{C}$ platform. A reference electrode was placed subcutaneously in the scalp and silver wire electrodes were positioned above the cornea, with contact being made by methylcellulose solution. Light flashes were presented by placing the mouse in a Ganzfield apparatus. Corneal evoked potentials were collected using UTAS-E4000 Visual Electrodiagnostic Test System and

EMWIN 8.1.1 software (LKC Technologies). Background light $\left(30 \mathrm{~cd} \cdot \mathrm{m}^{-2}\right)$ was presented for 10 min before recording flicker responses in the presence of the background light. Representative waveforms are shown.

\section{Immunoblotting}

Flash frozen retinal samples were solubilized in $1 \times$ PBS containing protease inhibitor (Pierce protease inhibitor ${ }^{\mathrm{TM}}$, Thermo scientific) using sonication for 4 pulses of 20 milliseconds at power setting 6 (Misonix XL-2000). The protein concentration was estimated with a NanoDrop (Thermo Scientific) spectrophotometer. Protein samples $(150 \mu \mathrm{g})$ were sizefractionated on 4-20\% Criterion (Bio-Rad) polyacrylamide gels. Proteins were then transferred to Immobilon-FL membrane (Millipore) and incubated with primary antibodies against specific proteins. The following antibodies were used: anti-PDE6 $\alpha$ (Cytosignal), anti-PDE6 $\beta$ (Thermo

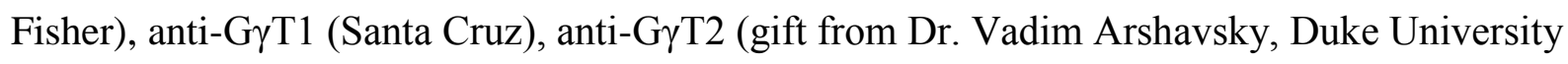

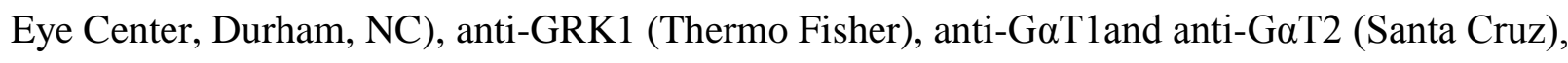
anti-GBT1 (Santa Cruz), anti-arrestin (Affinity Bioreagents), and anti-PDE6 $\alpha$ ' (Thermo Fisher). To detect primary antibodies, we used Odyssey goat anti-rabbit Alexa 680 and Odyssey goat 
anti-mouse Alexa 680 secondary antibodies (LI-COR Biosciences) at 1: 50,000 dilutions. Membranes were scanned with an Odyssey Infrared Imaging System (LI-COR Biosciences). Images are representative from three or more independent experiments.

\section{Immunohistochemistry.}

Mice were euthanized by $\mathrm{CO}_{2}$ inhalation, and eyes were enucleated. A 2-mm hole was made at the corneal limbus and eyes were fixed with $4 \%$ paraformaldehyde for 10 min before dissecting the anterior chamber and removing the lens. Eyecups for cryosections were then fixed for 50 min in $4 \%$ paraformaldehyde in PBS solution before cryoprotection in $20 \%$ sucrose overnight at $4{ }^{\circ} \mathrm{C}$. Eyecups were embedded in Tissue-Tek optimal cutting temperature compound (Sakura) and fast-frozen in dry ice ethanol bath. Blocks were sectioned with a Leica CM1850 Cryostat and $18 \mu \mathrm{m}$ sections were mounted on Superfrost plus slides. Cryosections were washed in PBS solution, and then incubated in blocking buffer [2\% goat serum (Invitrogen), 0.1\% Triton $\mathrm{X}-100$, and $0.05 \%$ sodium azide in PBS solution] for $1 \mathrm{~h}$. Primary antibodies were incubated for $4 \mathrm{~h}$ at room temperature or overnight at $4{ }^{\circ} \mathrm{C}$. Excess antibody was removed by three $10 \mathrm{~min}$ washes in PBS solution with $0.1 \%$ Triton X-100 before incubation with secondary antibody for $45 \mathrm{~min}$ at room temperature. Slides were washed twice for $10 \mathrm{~min}$ with PBS solution with $0.1 \%$ Triton X-100 and for 10 min in PBS solution. ProLong Gold antifade reagent (Invitrogen) was applied to each section, and then coverslips were mounted. Images were collected on a Zeiss LSM 510 Meta confocal microscope using 488-, 543-, and 633-nm laser lines. The following antibodies were used: anti-CNGA1/3 (University of California, Davis/ National Institutes of Health NeuroMab Facility), anti-PDE $\beta$ (Affinity Bio reagents), anti-G $\gamma$ T1 (Santa Cruz), antiG $\gamma$ T2 (gift from Dr. Vadim Arshavsky, Duke University Eye Center, Durham, NC), anti-PDE6 $\alpha^{\prime}$ (Thermo Fisher), anti-GRK1 (Ching-Kang Chen, Virginia Common wealth University, 
Richmond, VA), TO-PRO-3 nuclear stain (Invitrogen) was added to dilutions of Alexa Fluor secondary antibodies (Invitrogen) in antibody dilution buffer $(0.05 \%$ goat serum, $0.1 \%$ Triton $\mathrm{X}$ 100 , and $0.05 \%$ sodium azide in $1 \times$ PBS solution.

\section{Membrane fractionation}

Cell fractionation was performed as described (Kolandaivelu et al. 2014). Briefly, flashfrozen retinal samples were homogenized in $1 \times$ PBS containing protease inhibitors (Pierce protease inhibitor, Thermo scientific) by sonication with 5 pulses of 15 milliseconds at power setting 6 (Misonix XL-2000). After centrifugation at $5000 \times g$ for $5 \mathrm{~min}$ at $4^{\circ} \mathrm{C}$, the supernatant (total fraction) was collected and cellular debris was discarded. The low speed supernatant was then spun at $45000 \times g$ for 30 min (Rotor TLA-55 Beckman Coulter) to isolate the soluble fraction. After removal of the high-speed supernatant (cytosol soluble fraction), the pellet was re-suspended in equal volume of $1 \times$ PBS (membrane fraction). All protein samples were then analyzed by SDS-PAGE followed by immunoblotting to check for the distribution of cytosolic and membrane proteins. 


\section{RESULTS}

\section{Generating mice lacking Icmt expression in the retina}

To study the role of methyl esterification of proteins in the retina, we generated retinaspecific Icmt knockout mice. Six3-Cre expression eliminates Icmt expression in the retina and the forebrain at embryonic day 9.5 (Furuta et al. 2000, Christiansen et al. 2011). Icmf fl/wtSix3-Cre males (hereafter referred to as Icmt ${ }^{+/-}$mice) were mated with $I c m t f l / f l$ females to generate Icmf ${ }^{\mathrm{f} l / \mathrm{fl} \text { Six } 3-C r e}$ mice (hereafter referred to as Icmt ${ }^{-1-}$ mice, as well as littermate controls

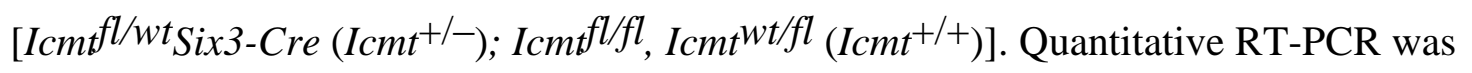
performed to confirm the absence of Icmt transcripts in the retina of $\mathrm{Icmt}^{-1-}$ mice (Fig. $2 \mathrm{~A}$ ). For these studies, $\mathrm{Icmt}^{+/-}$or $\mathrm{Icmt}^{+/+}$littermates were used as controls; those mice were similar to C57BL/6J animals with identical photoreceptor responses (not shown).

\section{ICMT-mediated protein processing is not required for photoreceptor morphogenesis}

To test the role of ICMT-mediated protein processing on photoreceptor development, we assessed the presence of rods and cones with cell-specific markers (Fig. 2 B). Rod and cone photoreceptor cells were present in a normal distribution in retinas of $\mathrm{Icmt}^{-}-$mice as shown by the presence of cyclic nucleotide gated channel (CNGA1/3, a marker for rod and cone cells) and peanut agglutinin (PNA, a marker for cone cells) (Fig. 2 B, top and bottom panels). Photoreceptor morphology appeared normal by electron microscopy (Data not shown). In addition, rod opsin (RHO) labeling demonstrated normal development of rod outer segments (Fig. 2 C). We did not observe any changes in the thickness of the outer nuclear layer (ONL) at P30 (Fig. 2 D, top panel). However, photoreceptors exhibited a slow and progressive loss of 
three to four nuclear layers (Student's $t$-test, P < 0.005) by P160 (Fig. 2 D bottom panel and Fig. $2 \mathrm{E})$. Altogether, our data show that methylation of isoprenylated proteins is not required for photoreceptor development.

\section{Light-evoked response is progressively reduced in retinas of Icmt-deficient mice}

Electroretinography (ERG) was used to measure the response of photoreceptor cells in Icmt ${ }^{+/}$and $\mathrm{Icmt}^{-\mathrm{I}}$ - mice to flashes of increasing light intensities. The $a$-wave of scotopic ERGs is generated by hyperpolarization of rod photoreceptor cells in response to light. Subsequent signaling to downstream neurons leads to depolarization of post-synaptic bipolar cells, which is measured by the $b$-wave of ERGs. At P24, there were no significant changes in rod or cone responses in mice lacking $I c m t$. This finding is in agreement with our observation that photoreceptor development is normal in $\mathrm{Icmt}^{-1-}$ mice. However, as the mice age, rod responses progressively declined. At P160, maximal rod responses were reduced by $82 \%$ ( $n=4$, Student's $t$-test, $P=0.032)$ and cone responses were reduced by $92 \%$ (Fig. 3$)(n=4$, Student's $t$-test, $P=$ 0.017). We also plotted the loss of visual response at various ages, which illustrated progressive loss of photoreceptor function over time (Fig. $3 \mathrm{C}$ and D). In comparison with rods, cones exhibit greater functional loss and were more sensitive to ICMT deficiency (Fig. $3 \mathrm{C}$ and D). We also checked the sensitivity of ERG response at different light intensities (Fig. 3 E and F). Strikingly, we did not observe any significant changes in light sensitivity at P24. At P160, the sensitivity of ERG response showed a slight reduction in mice lacking ICMT. Collectively, these results show that rod and cone signal transduction pathways are disrupted in aged $\mathrm{Icmt}^{-}{ }^{-}-$mice.

\section{Levels of a subset of isoprenylated photoreceptor proteins were reduced in the absence of ICMT-mediated protein processing}


We reasoned that a reduced visual response might result from lower levels of the isoprenylated proteins involved in phototransduction. Therefore, we assessed protein levels by Western blotting with retinal extracts from $I c m t^{+/-}$and $I c m t^{-1-}$ mice at P30 (before significant retinal degeneration was evident). We observed more than a $60 \%$ reduction $(n=4$, Student's $t$ test, $P=0.0011)$ in levels of the cone PDE6 $\left(\mathrm{PDE} \alpha^{\prime}\right)$ and rod and cone transducin- $\gamma$ subunits

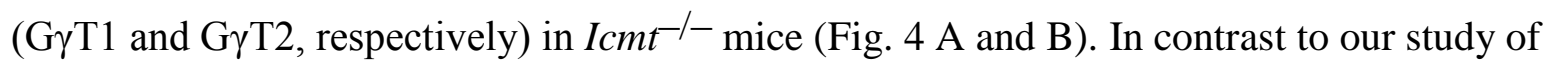
retina-specific Rcel knockout mice, we did not observe any changes in the electrophoretic mobility of isoprenylated proteins (Christiansen et al. 2011). Surprisingly, the levels of isoprenylated rod PDE6 catalytic subunits (PDE6 $\alpha \beta)$ and G-protein receptor kinase-1 (GRK1) were unaffected. As expected, rod arrestin (rARR), a photoreceptor protein that is not isoprenylated, was unaffected (Fig. 4 A and B). The transducin $\alpha(\mathrm{G} \alpha \mathrm{T} 1)$ subunits, which form a heterotrimeric complex with G $\beta 1-\mathrm{G} \gamma \mathrm{T} 1$, were reduced in the absence of ICMT-mediated protein methylation. We also assessed protein levels at P16 (well after Six3-Cre-mediated recombination, which occurs at E9.5) and did not observe significant changes (not shown). Our results suggest that lack of cysteine methylation by ICMT causes photoreceptor $C A A X$ proteins to undergo increased protein turnover, with transducin subunits and PDE6 $\alpha$ ' showing the most pronounced reductions in their steady-state levels.

\section{Membrane association of transducin and cone PDE6 is affected in retinas lacking Icmt expression}

We hypothesized that impaired association of unmethylated G $\gamma \mathrm{T} 1 / 2$ and PDE6 $\alpha^{\prime}$ with membranes might contribute to their lower levels in $I c m t^{-/-}$mice. To examine the effect of ICMT-mediated methylation on membrane attachment of proteins, we performed isotonic 
membrane fractionation of retinal extracts. Our results show that the majority (77\%) of GyT1 in control $\left(\mathrm{Icmt}^{+/-}\right)$mice was present in the membrane fraction. In contrast, less than $10 \%$ of G $\gamma \mathrm{T} 1$ was in the membrane fraction in $I c m t^{-}-$mice $(n=4$, Student's $t$-test, $P=0.042)$. The membrane attachment of G $\beta \mathrm{T} 1$, a partner of $\mathrm{G} \gamma \mathrm{T} 1$, was similarly affected (Fig. 5 A and B). A

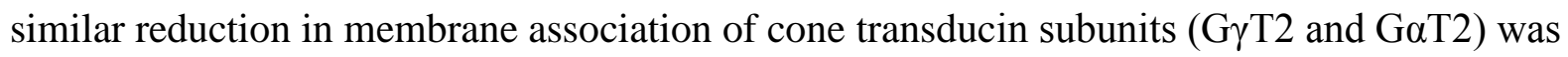
also detected. Defective membrane association of cone PDE6 $\alpha^{\prime}$ and geranylgeranylated rod PDE6 $\beta$ in Icmt $^{-1-}$ animals was also observed (Fig. 5 A and B). Surprisingly, the membrane association of GRK1 and PDE6 $\alpha$, which are farnesylated proteins, was not affected by the absence of methylation. Guanylate cyclase-1 (RetGC1) and aryl hydrocarbon receptor interacting protein like-1 (AIPL1) were used as membrane-bound and cytosolic protein controls, respectively, and were unaffected by the deficiency in ICMT-mediated protein processing (Fig. 5). Overall, our results demonstrate the importance of methylation in altering the membrane binding of the visual G-protein, transducin, and PDE6.

\section{Trafficking of isoprenylated OS proteins are not affected by the absence of ICMT- mediated methylation}

Our earlier study revealed the importance of RCE1-mediated endoproteolysis in retinal neurons (the processing step that precedes the ICMT-mediated methylation step). We found that the PDE6 holoenzyme is assembled, but does not traffic to the OS in the $R c \mathrm{I}^{-\mathrm{I}}$ mice (Christiansen et al. 2011). The defective transport of PDE6 could be due to lack of endoproteolysis, the absence of the subsequent methylation step, or to the absence of both steps. To distinguish between these possibilities, we examined the trafficking of PDE6 and other isoprenylated OS proteins in Icmt-deficient mice. Immunolocalization of PDE6 $\beta$ was compared

to that of cyclic nucleotide gated channel (CNGA1/3), a marker of OS (Fig. 6 A and B). Merged 
images demonstrated that PDE6 was transported to the OS and colocalized with CNGA1/3 in retinas lacking Icmt expression. Similarly, G $\gamma \mathrm{T} 1$ was localized in the OS with CNGA1/3 (Fig. 6 B). In addition, no significant defects in localization of cone specific PDE6 $\alpha^{\prime}, \mathrm{G} \gamma \mathrm{T} 2$, and GRK1 (present in both rods and cones) were observed at P30 (Fig. $6 \mathrm{C}$ and D).

Despite marked reduction in photopic ERG response at P160, cone density in Icmtdeficient retina was unaltered. Interestingly, GRK1 was severely reduced in cones while expression of GRK1 in rods did not show any significant changes (Fig. 7B). A drastic reduction in cone PDE6 and G $\gamma$ T2 levels was also observed at P160 in Icmt-deficient mice (Fig. 7A and B). The results from immunolocalization studies were supported by our immunoblotting analysis (Fig. 7 C) and demonstrate the importance of methylation for the stability of isoprenylated proteins. Our results show that methylation of proteins has little or no effect on the ability of isoprenylated proteins to traffic to the OS but it is crucial for the stability of isoprenylated OS proteins. 


\section{DISCUSSION}

Our current studies highlight the importance of posttranslational modifications of proteins in the function and survival of photoreceptor neurons. The most significant defect in mice lacking ICMT-mediated protein methylation in the retina is the loss of visual function accompanied by slow photoreceptor degeneration.

Methylation of proteins by Icmt was not required for retinal development, despite early inactivation of Icmt in the retina. The characteristic layering of neurons in the retina was preserved, and all retinal layers were formed normally. In addition, we found no alteration in the ultrastructure of rod and cone photoreceptors by electron microscopy (Data not shown). These findings are in agreement with normal photoreceptor ERG response at P24. However, we observed progressive reduction in rod and cone responses with extinguished photoreceptor responses by 5 months of age. There appeared to be discordance between the striking extent of functional deficit and the loss of only 3-4 layers of photoreceptor nuclei at 5 months. We attribute the defective rod response to loss of transducin, while the cone responses were affected by the loss of both transducin and phosphodiesterase, two crucial players in phototransduction. This interpretation is supported by studies where elimination of transducin (G $\gamma \mathrm{T} 1)$ expression resulted in late-onset retinal degeneration and reduced rod ERG responses (72). A mouse model defective in cone PDE6 showed similar reduction in photopic ERG (Chang et al. 2006, Kolandaivelu et al. 2011). Interestingly, the survival of the neurons in the inner retinal layer (INL) was unaffected by the absence of Icmt expression.

The effect of ICMT-mediated protein methylation on the stability of isoprenylated proteins is variable (156). For instance, RhoA undergoes higher rates of turnover in the absence of methylation, whereas K-Ras is more stable in the absence of methylation (55). The levels of 
cone PDE6 $\alpha^{\prime}$ in $\mathrm{Icmt}^{-1-}$ mice were reduced significantly (60-70\%), in contrast to normal expression of rod PDE6 prior to degeneration at P30 (Fig.4). Interestingly, as the photoreceptors degenerate by four nuclear layers at P160, we observed a major reduction in several phototransduction proteins such as rod and cone PDE6, cone GRK1 and transducin (Fig.7). Along with PDE6, our immunolocalization studies showed markedly reduced levels of GRK1 in cones (Fig.7). The reason for the selective reduction in cone PDE6 subunits prior to degeneration is not clear. Cone PDE6 subunits are geranylgeranylated, whereas the obligatory catalytic heteromer of rod PDE6 is farnesylated $(\alpha)$ or geranylgeranylated $(\beta)$ (Anant et al. 1992). Similarly, the reason for the selective reduction in farnesylated GRK1 in cones at P160 is not clear. It is known that methylation of PDE6 is crucial for its interaction with PrBP $\delta$, a prenylbinding protein, and is needed for stability and trafficking of PDE6 and GRK1 in photoreceptors (Zhang et al. 2007; Zhang et al. 2012, Cook et al. 2000). Overall, our data support a model where methylation of cone PDE6 and GRK1 is essential for the interaction of these proteins with $\operatorname{PrBP} \delta$, and the absence of this interaction in Icmt-deficient cones leads to reduced cone photoreceptor function.

The results from this study also show a requirement for protein methylation in maintaining normal levels of the G-protein transducin complex $(\alpha \beta \gamma)$. Unmethylated transducin

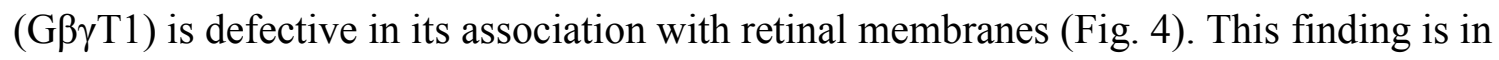
agreement with a previous in vitro study that demonstrated the importance of protein methylation in anchoring of G $\beta \gamma \mathrm{T} 1$ to rod outer segment membranes (Fukada et al. 1990, Ohguro et al. 1991). However, the membrane fractionation pattern of GaT1, a myristoylated protein, was unaltered, suggesting that the assembly of the G-protein transducin complex is affected in the absence of methylation. The reduced rod response is likely a result of deficiencies in both 
transducin complex formation and inadequate membrane association of G $\beta \gamma \mathrm{T} 1$. The puzzling finding in our studies was the normal photo-responses at P24 despite defective G $\gamma \mathrm{T} 1$ membrane association. One explanation for the progressive loss of rod ERGs could be the accumulation of soluble unmethylated G $\beta \gamma \mathrm{T} 1$ in the rod OS over time that interferes with $\mathrm{RHO}^{*}$-mediated light signaling.

Phototransduction proteins are synthesized, assembled in the IS, and then transported to OS. However, methylation of isoprenylated proteins does not affect the ability of phototransduction proteins to traffic to the OS. This observation stands in contrast to our findings with Rce $^{-{ }^{-}}$mice, where rod and cone PDE6 accumulated in the IS. Based on these results, we conclude that the retention of the $-A A X$ in PDE6 catalytic subunits in the setting of RCE1 deficiency impairs PDE6 transport to the OS. Our findings showing severe retinal disease phenotypes in $\mathrm{RceI}^{-{ }^{-}}$mice also stand in contrast to published reports on global Icmt and Rcel knockout mice. A global deficiency of Icmt results in embryonic death much earlier in development than a deficiency of Rce $1(17,45,53,55)$. Although it seems unlikely, we cannot rule out the possibility that a distinct methyltransferase might retain the capacity to methylate certain proteins after inactivation of Icmt in the retina. If there were another methyltransferase, it could potentially help to explain the fact that retinal phenotypes were less severe in mice lacking ICMT than in mice lacking RCE1, despite the fact that ICMT has a larger number of protein substrates (CAAX proteins plus CXC Rab proteins).

Overall, the current investigations revealed that ICMT-mediated protein methylation is essential for stability and membrane anchorage of isoprenylated G $\beta \gamma \mathrm{T} 1$ and cone PDE6 and is required for photoreceptor function and survival. 


\section{REFERENCES}

1. Anant JS , Ong OC, Xie HY, Clarke S, O'Brien PJ, Fung BK. (1992). "In vivo differential prenylation of retinal cyclic GMP phosphodiesterase catalytic subunits." J Biol Chem 267(2): 687-90.

2. Bergo MO, Gavino BJ, Hong C, Beigneux AP, McMahon M, Casey PJ, Young SG. (2004). "Inactivation of Icmt inhibits transformation by oncogenic K-Ras and B-Raf." J Clin Invest 113(4): 539-50.

3. Bergo MO, Leung GK, Ambroziak P, Otto JC, Casey PJ, Gomes AQ, Seabra MC, Young SG. (2001). "Isoprenylcysteine carboxyl methyltransferase deficiency in mice." J Biol Chem 276(8): 5841-5.

4. Bergo MO, Leung GK, Ambroziak P, Otto JC, Casey PJ, Young SG (2000). “Targeted inactivation of the isoprenylcysteine carboxyl methyltransferase gene causes mislocalization of K-Ras in mammalian cells". J Biol Chem. 9;275(23):17605-10.

5. Chang B, Dacey MS, Hawes NL, Hitchcock PF, Milam AH, Atmaca-Sonmez P, Nusinowitz S, Heckenlively JR (2006). "Cone photoreceptor function loss-3, a novel mouse model of achromatopsia due to a mutation in Gnat2". Invest Ophthalmol Vis Sci. 47(11): 5017-21.

6. Christiansen JR, Kolandaivelu S, Bergo MO, Ramamurthy V (2011). "RAS-converting enzyme 1-mediated endoproteolysis is required for trafficking of rod phosphodiesterase 6 to photoreceptor outer segments." Proc Natl Acad Sci U S A.

7. Cook TA, Ghomashchi F, Gelb MH, Florio SK, Beavo JA. (2000). "Binding of the delta subunit to rod phosphodiesterase catalytic subunits requires methylated, prenylated Ctermini of the catalytic subunits." Biochemistry 39(44): 13516-23.

8. Court H, Amoyel M, Hackman M, Lee K, Xu R, Miller G, Bar-Sagi D, Bach E, Bergö M, and Philips M (2013), "Isoprenylcysteine carboxylmethyltransferase deficiency exacerbates KRAS-driven pancreatic neoplasia via Notch suppression" J Clin Invest.; 123(11): 4681-4694.

9. Deng WT, Sakurai K, Liu J, Dinculescu A, Li J, Pang J, Min SH, Chiodo VA, Boye SL, Chang B, Kefalov VJ, Hauswirth WW. (2009). "Functional interchangeability of rod and cone transducin alpha-subunits." Proc Natl Acad Sci U S A 106(42): 17681-6.

10. Fukada YS, Takahiko Matsuda, Koichi Kokame, Toshifumi Takaos, Yasutsugu Shimonishi§, Toyoaki Akinon and TBru Yoshizawall (1994) "Effects of Carboxyl Methylation of Photoreceptor G Protein y-Subunit in Visual Transduction* J, biol. chem. Vol. 269, No. 7,pp. 5163-5170, 1994. 
11. Fukada Y, Takao T, Ohguro H, Yoshizawa T, Akino T, Shimonishi Y (1990). "Farnesylated gamma-subunit of photoreceptor G protein indispensable for GTPbinding." Nature. 16; 346(6285):658-60.

12. Furuta Y, Lagutin O, Hogan BL, Oliver GC. (2000). "Retina- and ventral forebrainspecific Cre recombinase activity in transgenic mice." Genesis 26(2): 130-2.

13. Griggs, A. M., K. Hahne, et al. (2010). "Functional oligomerization of the Saccharomyces cerevisiae isoprenylcysteine carboxyl methyltransferase, Ste14p." J Biol Chem 285(18): 13380-7.

14. Hrycyna, C. A., S. K. Sapperstein, et al. (1991). "The Saccharomyces cerevisiae STE14 gene encodes a methyltransferase that mediates $\mathrm{C}$-terminal methylation of a-factor and RAS proteins." EMBO J 10(7): 1699-709.

15. Inglese, J, Koch WJ, (1992). "Isoprenylation in regulation of signal transduction by Gprotein-coupled receptor kinases." Nature 359(6391): 147-50.

16. Kim E, Ambroziak P, Otto J C, Taylor B, Ashby M, Shannon K, Casey PJ, Young SG (1999). "Disruption of the mouse Rcel gene results in defective Ras processing and mislocalization of Ras within cells". J Biol Chem. 26; 274 (13):8383-90.

17. Kolandaivelu S, Singh R, and Ramamurthy V (2014)," AIPL1, A protein linked to blindness, is essential for the stability of enzymes mediating cGMP metabolism in cone photoreceptor cells' Hum Mol Genet. 23(4): 1002-1012.

18. Kolandaivelu S, Chang B, Ramamurthy V (2011). "Rod phosphodiesterase-6 (PDE6) catalytic subunits restore cone function in a mouse model lacking cone PDE6 catalytic subunit”. J Biol Chem. 286(38):33252-9.

19. Kolandaivelu S, Huang J, Hurley JB, Ramamurthy V (2009). "AIPL1, a protein associated with childhood blindness, interacts with alpha-subunit of rod phosphodiesterase (PDE6) and is essential for its proper assembly." J Biol Chem 284(45): 30853-61.

20. Kolesnikov AV, Rikimaru L, Hennig AK, Lukasiewicz PD, Fliesler SJ, Govardovskii VI, Kefalov VJ, Kisselev OG. (2011). "G-protein betagamma-complex is crucial for efficient signal amplification in vision." J Neurosci 31(22): 8067-77.

21. Lane KT and Beese LS (2006). "Thematic review series: lipid posttranslational modifications. Structural biology of protein farnesyltransferase and geranylgeranyltransferase type I." J Lipid Res 47(4): 681-99. 
22. Lobanova ES, Finkelstein S, Herrmann R, Chen YM, Kessler C, Michaud NA, Trieu LH, Strissel KJ, Burns ME, ArshavskyVY (2008). "Transducin gamma-subunit sets expression levels of alpha- and beta-subunits and is crucial for rod viability". J Neurosci. 26; 28(13):3510-20.

23. Lyubarsky, AL and Pugh EN, Jr. (1996). "Recovery phase of the murine rod photoresponse reconstructed from electroretinographic recordings." J Neurosci 16(2): 563-71.

24. Michaelson D, Ali W, Chiu VK, Bergo M, Silletti J, Wright L, Young SG, Philips M (2005). "Postprenylation CAAX processing is required for proper localization of Ras but not Rho GTPases." Mol Biol Cell. Apr;16(4):1606-16.

25. Moussaif M, Rubin WW, Kerov V, Reh R, Chen D, Lem J, Chen CK, Hurley JB, Burns M E, Artemyev N O (2006). "Phototransduction in a transgenic mouse model of Nougaret night blindness." J Neurosci 26(25): 6863-72.

26. Ohguro H, Fukada Y, Takao T, Shimonishi Y, Yoshizawa T, Akino T (1991). Carboxyl methylation and farnesylation of transducin gamma-subunit synergistically enhance its coupling with metarhodopsin II.” EMBO J. 10(12):3669-74.

27. Ong OC, Ota IM, Clarke S, Fung BK. (1989). "The membrane binding domain of rod cGMP phosphodiesterase is posttranslationally modified by methyl esterification at a Cterminal cysteine." Proc Natl Acad Sci U S A 86(23): 9238-42.

28. Parish CA and Rando RR (1996). "Isoprenylation/methylation of proteins enhances membrane association by a hydrophobic mechanism." Biochemistry 35(26): 8473-7.

29. Perez-Sala D., Tan EW, Cañada FJ, Rando RR. (1991). "Methylation and demethylation reactions of guanine nucleotide-binding proteins of retinal rod outer segments." Proc Natl Acad Sci U S A 88(8): 3043-6.

30. Sokolov M, Lyubarsky AL, Strissel KJ, Savchenko AB, Govardovskii VI, Pugh EN Jr, Arshavsky VY. (2002). "Massive light-driven translocation of transducin between the two major compartments of rod cells: a novel mechanism of light adaptation." Neuron 34(1): 95-106.

31. Svensson AW, Casey PJ, Young SG, Bergo MO. (2006). "Genetic and pharmacologic analyses of the role of Icmt in Ras membrane association and function." Methods Enzymol 407: 144-59.

32. Swanson RJ and Applebury ML (1983). "Methylation of proteins in photoreceptor rod outer segments." J Biol Chem 258(17): 10599-605. 
33. Zhang H, Constantine R, Frederick JM, Baehr W (2012) "The prenyl-binding protein $\operatorname{PrBP} / \delta$ : a chaperone participating in intracellular trafficking”. Vision Res. $15 ; 75: 19-25$.

34. Zhang XM, Chen BY, Ng AH, Tanner JA, Tay D, So KF, Rachel RA, Copeland NG, Jenkins NA, Huang JD. (2005). "Transgenic mice expressing Cre-recombinase specifically in retinal rod bipolar neurons." Invest Ophthalmol Vis Sci 46(10): 3515-20.

35. Zhang H, Li S, Doan T, Rieke F, Detwiler PB, Frederick JM, Baehr W (2007) "Deletion of PrBP/delta impedes transport of GRK1 and PDE6 catalytic subunits to photoreceptor outer segments". Proc Natl Acad Sci U S A. 22;104 (21):8857-62. 


\section{FIGURE LEGENDS AND FIGURES}

Fig. 1. Schematic representation of prenylation and postprenylation processing. Rod phosphodiesterase6 (PDE6 $\alpha$ ) is farnesylated. (1) Protein farnesyltransferase (FTase-I), a cytosolic protein, adds a farnesyl lipid (FPP) to the cysteine of the $C A A X(C A A X=C C I Q$ for PDE6 $\alpha$ ) motif. (2) RCE1-mediated endoproteolysis at the ER membrane, cleaves the last three amino acids of the $C C I Q$ motif (i.e., $-C I Q$ ). (3) ICMT, a ER membrane protein, catalyzes the methyl esterification of the farnesylcysteine. After methylation, prenylated proteins are extracted from ER membranes by proteins such as $\operatorname{PrBP} \delta$ and further transported to photoreceptor OS.

Fig. 2. Photoreceptor development is unaltered in the absence of Icmt expression. (A) RTPCR on P60 retinal cDNA showing the expression of Icmt (normalized to hypoxanthine phosphoribosyltransferase, $\operatorname{Hprt})(n=3$, Student's $t$-test, $P=0.033)$. Icmt primers were designed with the forward primer in exon 1 and reverse primer in exon 4. (B) Cryosections of P60 Icmt ${ }^{+/-}$ and $I c m t^{-1}$ - littermate mice labeled with cyclic-nucleotide gated channel alpha 1 (CNGA1/3 Green), peanut agglutinin (PNA-Red), and TO-PRO-3 (Blue). OS, Outer Segment; IS, Inner Segment; ONL, Outer Nuclear Layer. Scale $=5 \mu \mathrm{M}$. (C) Cryosections of P60 Icmt ${ }^{+/-}$and Icmt I- littermates labeled with rod opsin (RHO-Green), peanut agglutinin (PNA-Red), and TO-PRO3 (Blue). Scale $=10 \mu \mathrm{M}$. (D) Cryosections of P30 (top panel) and P160 (bottom panel) Icmt ${ }^{+/-}$ and $I c m t^{-}-$littermate mice labeled with propidium iodide (PI), a nuclear stain. (E) Spyder plot analysis of ONL thickness at P160 ( $n=4$, Student's $t$-test, $P<0.005)$. 
Fig. 3. Visual deficit in mice lacking Icmt in the retina.Rod (A) and cone (B) ERG responses recorded from P24 and P160 Icmt ${ }^{+/-}$and $\mathrm{Icmt}^{-/}$littermates $(n=4)$. (C and D) Amplitude of "a" and "b" waves versus age (in days). Rod responses (C) correspond to the amplitude of the "a" wave, whereas cone responses (D) reflect the amplitude of the "b" wave. Representative rod waveforms measured at $-0.8 \log \mathrm{cd}^{*} \mathrm{~s} / \mathrm{m}^{\wedge} 2$, while representative cone waveforms measured at $0.7 \log \mathrm{cd}^{*} \mathrm{~s} / \mathrm{m}^{\wedge} 2(n=4$, Student's $t$-test, $P<0.002) . \mathrm{P}$, postnatal days; ms, milliseconds; $\mu \mathrm{V}$, microvolts; $n$, number of littermates. (E and F) Intensity response relations of scotopic "a" waves at P24 and P160 Icmt ${ }^{+/-}$and Icmt ${ }^{-/-}$littermates $(n=4)$. The data were fitted with the hyperbolic functions that yielded scotopic "a" wave half-saturating light intensities of $0.10 \pm$ $0.02 \mathrm{~cd}^{*} \mathrm{sm}^{-2}$ for $\mathrm{Icmt}^{+/}$and $0.11 \pm 0.034 \mathrm{~cd}^{*} \mathrm{sm}^{-2}$ for $\mathrm{Icmt}^{-/}$littermates respectively at P24 (Panel E). The "a" wave maximum amplitudes were $413.22 \pm 14.32 \mu \mathrm{V}\left(\right.$ Icmt $^{+/-}$; $\left.\mathrm{n}=4\right)$ and $398.79 \pm 8.21 \mu \mathrm{V}\left(\right.$ Icmt $\left.^{-/-} ; \mathrm{n}=4\right)$. Similarly, at P160, data were fitted with the hyperbolic functions that yielded scotopic "a" wave half-saturating light intensities of $0.11 \pm 0.02 \mathrm{~cd}^{*} \mathrm{sm}^{-2}$

for $\mathrm{Icmt}^{+/}$and $0.24 \pm 0.12 \mathrm{~cd}^{*} \mathrm{sm}^{-2}$ for $\mathrm{Icmt}^{-/-}$littermates respectively at P160 (Panel F). The “a” wave maximum amplitudes were $461.17 \pm 16.11 \mu \mathrm{V}\left(\mathrm{Icmt}^{+/-} ; \mathrm{n}=4\right)$ and $90.12 \pm 13.21 \mu \mathrm{V}$ $\left(\right.$ Icmt $\left.^{-1-} ; \mathrm{n}=4\right)$. The values are mean \pm SEM.

Fig. 4. Levels of a subset of isoprenylated photoreceptor proteins are reduced in the absence of ICMT-mediated protein processing. Immunoblots using P30 retinal lysates. (A) Western blots of protein extracts probed with antibodies against the indicated proteins. (B) Quantitative comparison of indicated photoreceptor proteins between Icmt-deficient mice and littermate controls. The $y$ axis of the bar graph shows the $\%$ of the integrated intensity measurement normalized to measurements in $\mathrm{Icmt}^{+/-}$mice (as judged by Odyssey imaging 
software). Integrated intensity values were normalized to $\alpha$-tubulin $\left(n=4\right.$, Student's $t$-test; ${ }^{*} P<$ $0.004 ; * * P<0.002)$.

Fig. 5. Membrane association of transducin and cone PDE6 is impaired in retinae lacking Icmt expression. (A) Isotonic membrane fractionation of P30 retinal lysates, followed by Western blotting. AIPL1, RetGC1, and Cone Arrestin (cARR) serve as controls $(n=4)$. (B) Quantitative comparison of indicated proteins in membrane-bound and soluble fractions in Icmt knockout mice and littermate controls. The $y$ axis of the bar graph is the $\%$ of the integrated intensity measurements normalized to measurements in control $\left(\mathrm{Icmt}^{+-}\right)$samples, as judged with Odyssey imaging software. Integrated intensity values were normalized to $\alpha$-tubulin ( $n=4$, ** $P<0.05) . \mathrm{T}=$ total fraction; $\mathrm{S}=$ soluble fraction; $\mathrm{M}=$ membrane-bound fraction.

Fig. 6. Rod PDE6 and transducin do not require ICMT-mediated protein methylation to be transported to the OS. Immunofluorescent labeling of retinae from P30 $\mathrm{Icmt}^{+/-}$and $\mathrm{Icmt}^{-1-}$ littermates. (A) CNGA1/3 (red) labels the OS; PDE6 $\beta$-subunit is in green. B) Rod transducin $\gamma$

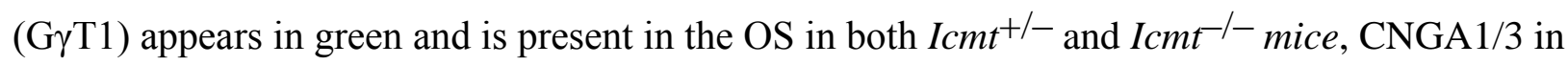
red labels the OS. C) Cone PDE6 (PDE6 $\alpha^{\prime}$ ) is green; GRK1 is red. D) PNA in red labels the cone sheath, G $\gamma \mathrm{T} 2$ is shown in green and colocalizes with PNA; nuclei are stained with TO-PRO-3 in blue. Scale $=10 \mu \mathrm{M}$.

Fig. 7. PDE6 and GRK1 levels in cones are reduced along with subset of other indicated proteins in mice lacking ICMT-mediated protein methylation. Immunofluorescent labeling of P160 Icmt ${ }^{+/-}$and Icmt ${ }^{-1-}$ littermates. (A) PNA in red labels the cone sheath; G $\gamma \mathrm{T} 2$ appears in green and colocalizes with PNA. Nuclei are stained with TO-PRO-3 in blue. (B) GRK1 is shown 
in red; cone PDE6 (PDE6 $\left.\alpha^{\prime}\right)$ appears in green. Both GRK1 and cone PDE6 are absent in Icmt ${ }^{-}-$ at $\mathrm{P} 160$. Scale $=10 \mu \mathrm{M}$. (C) Representative western blots of protein extracts probed with antibodies against the indicated proteins (left panel) and quantitative comparison of indicated proteins between $\mathrm{Icmt}^{-/}$- mice and littermate controls (right panel). The $y$ axis of the bar graph is the $\%$ of the integrated intensity measurements normalized to measurements in control $\left(\right.$ Icmt $\left.^{+/-}\right)$ samples, as judged with Odyssey imaging software $(n=4)$. Integrated intensity values are normalized to $\alpha$-tubulin $(n=4$, Student's $t$-test; $* P<0.05$; ** $P<0.02)$. 


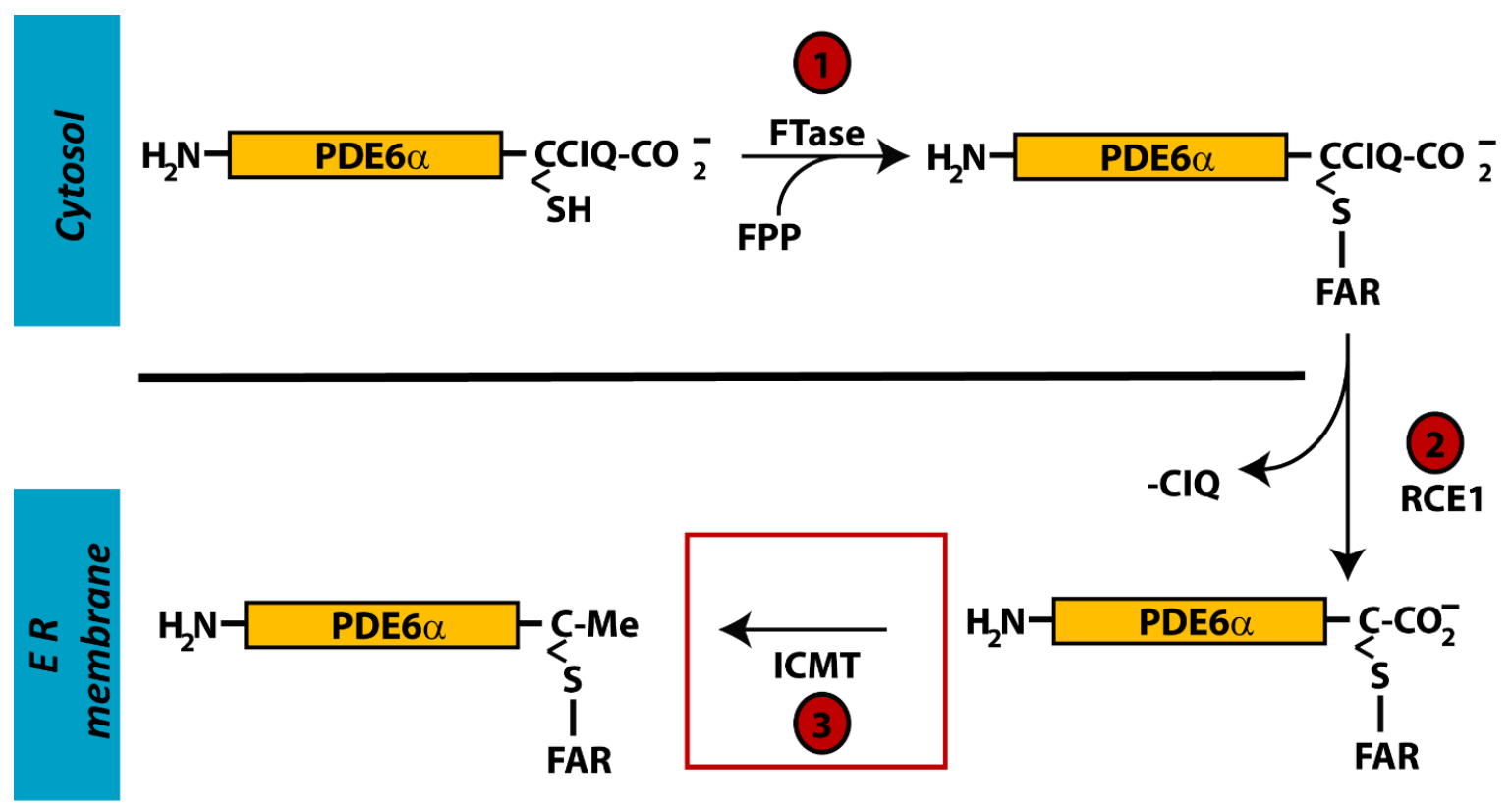

Figure 1 Schematic representation of prenylation and postprenylation processing. 


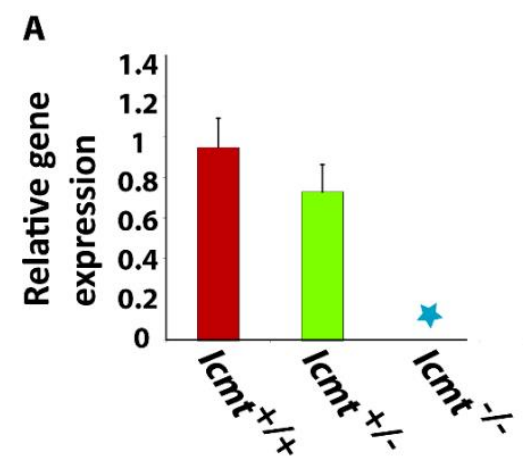

B
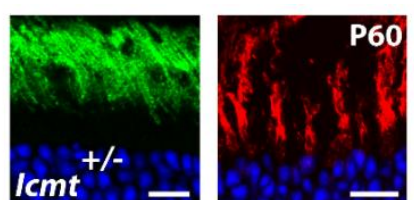

C
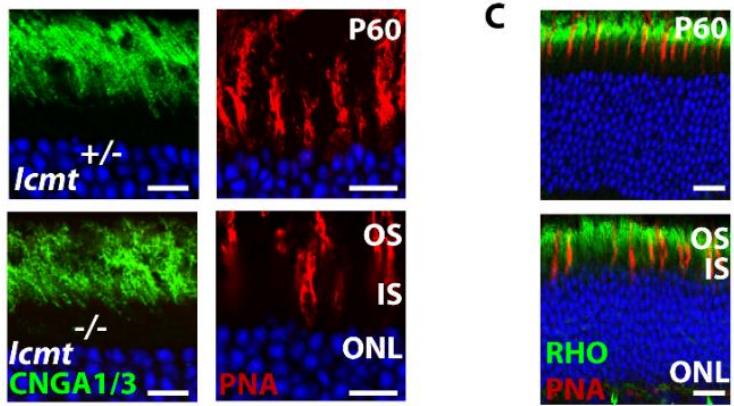

D
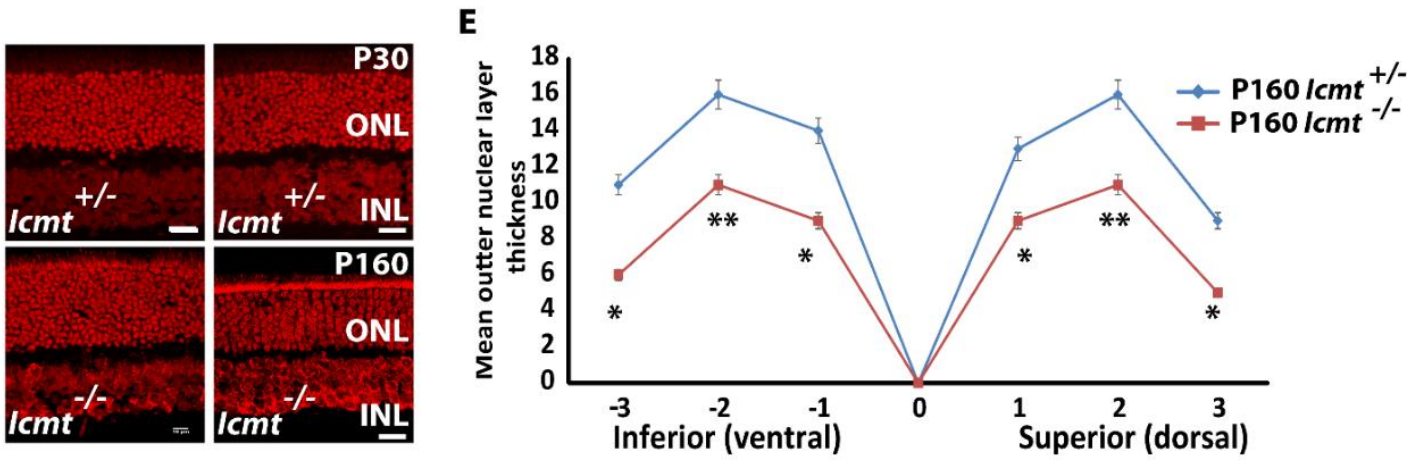

Figure 2 Photoreceptor development is unaltered in the absence of Icmt expression. 

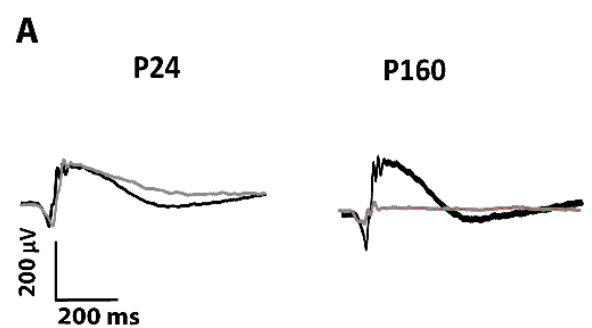

B

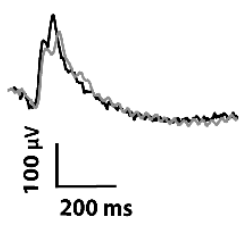

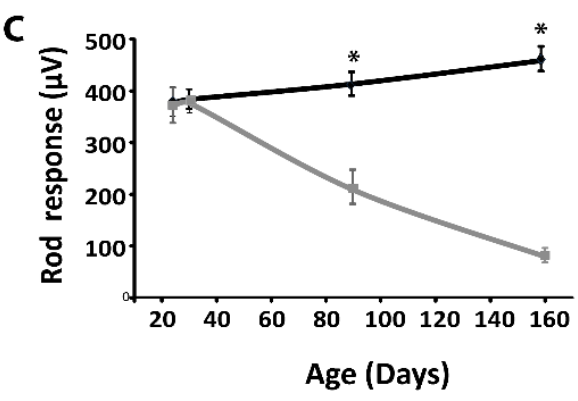

D
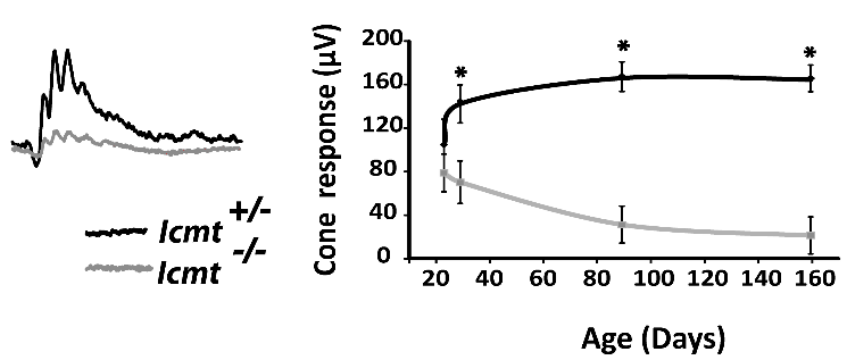
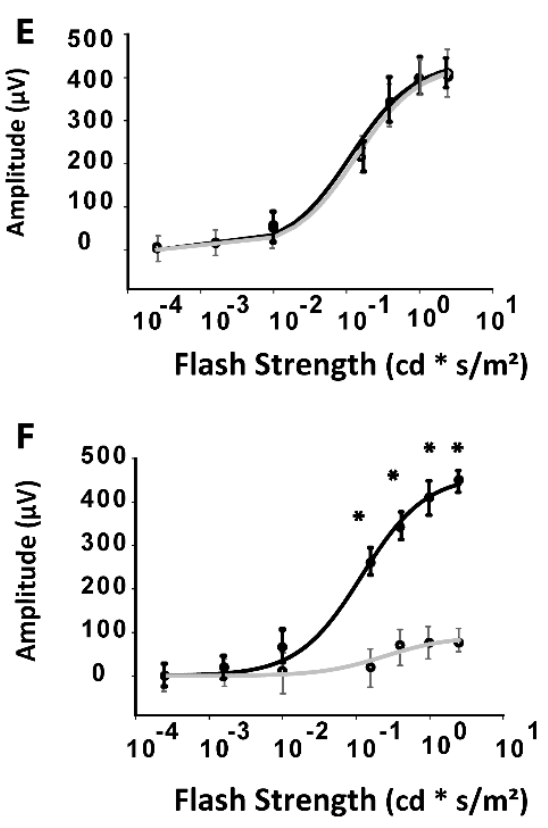

Figure 3 Visual deficit in mice lacking Icmt in the retina. 
A

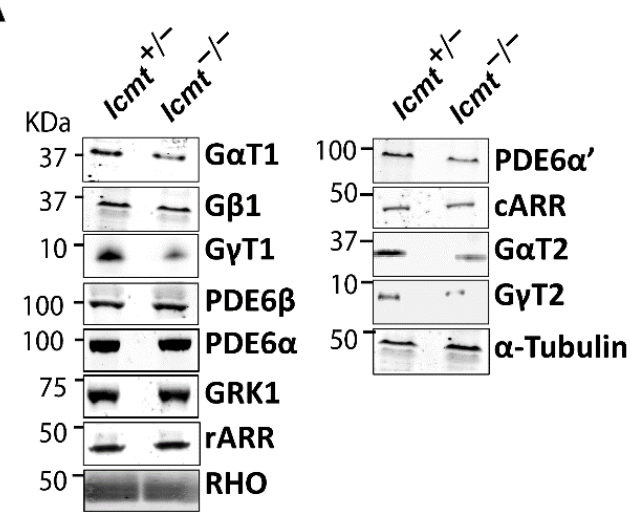

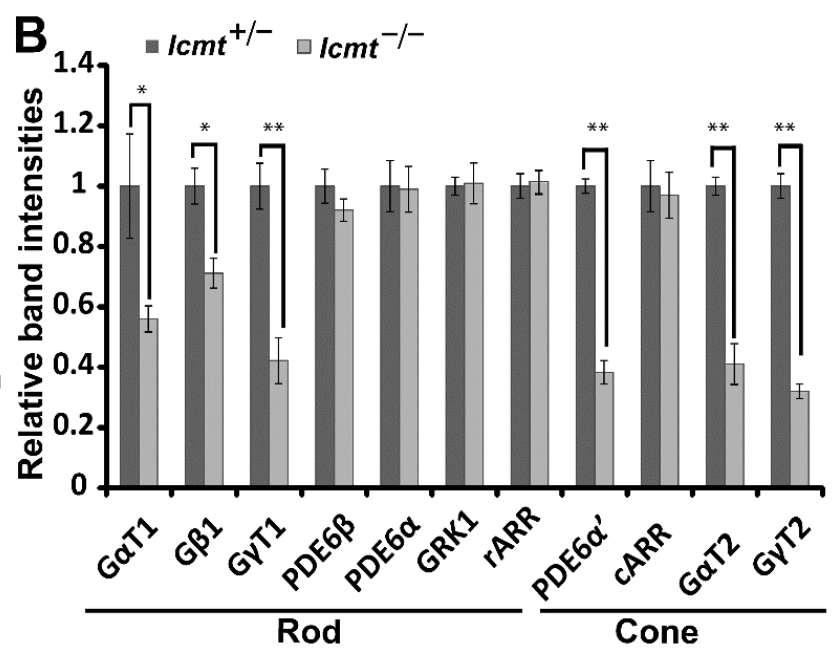

Figure 4 Levels of subset of isoprenylated proteins are reduced in retinas lacking Icmt expression. 

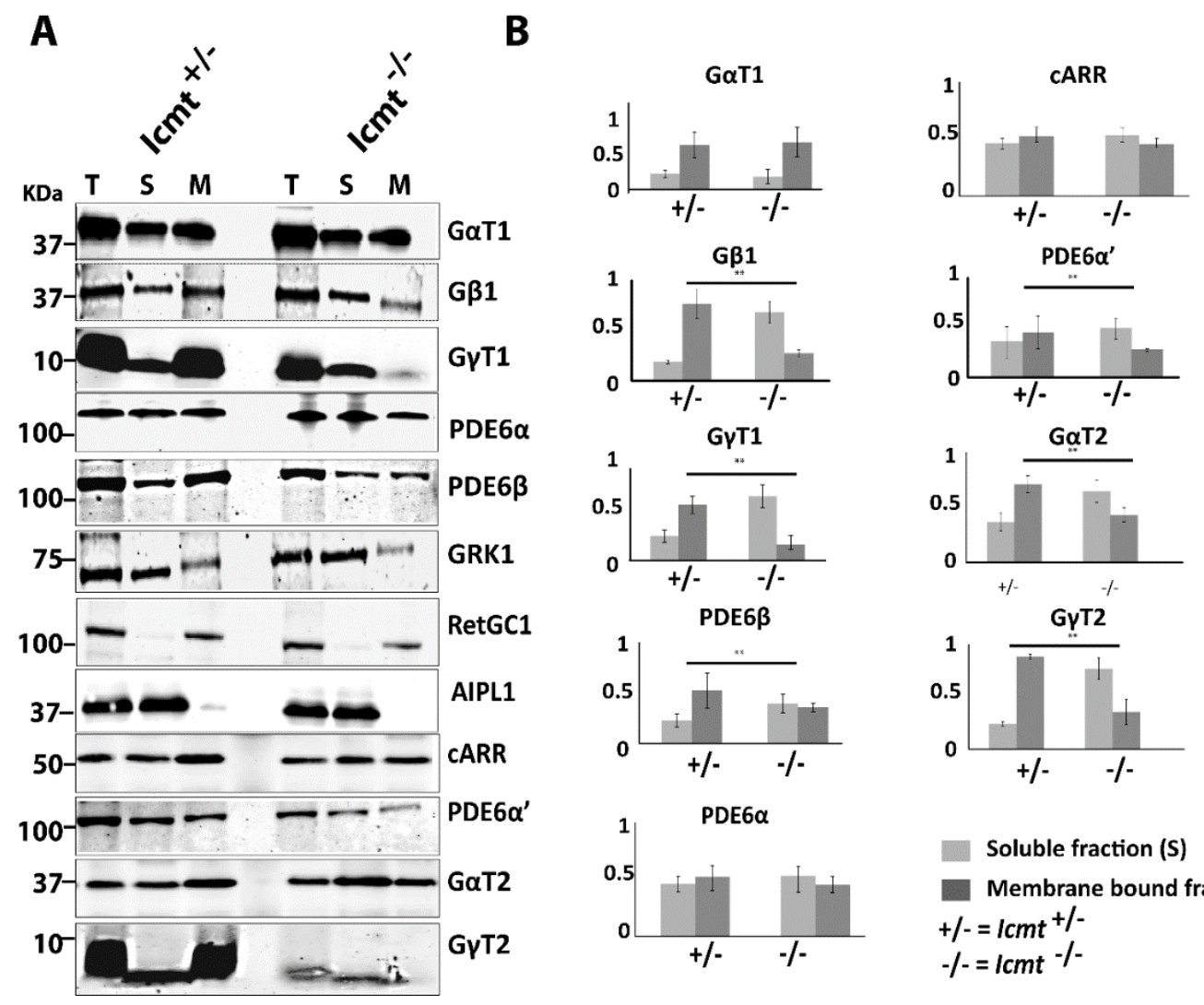

Soluble fraction (S)

Membrane bound fraction (M) $+/-=1 \mathrm{cmt}+$ $-/-=1 \mathrm{cmt}-1-$

Figure 5 Membrane association of transducin and cone PDE6 is impaired in retinas lacking Icmt expression. 


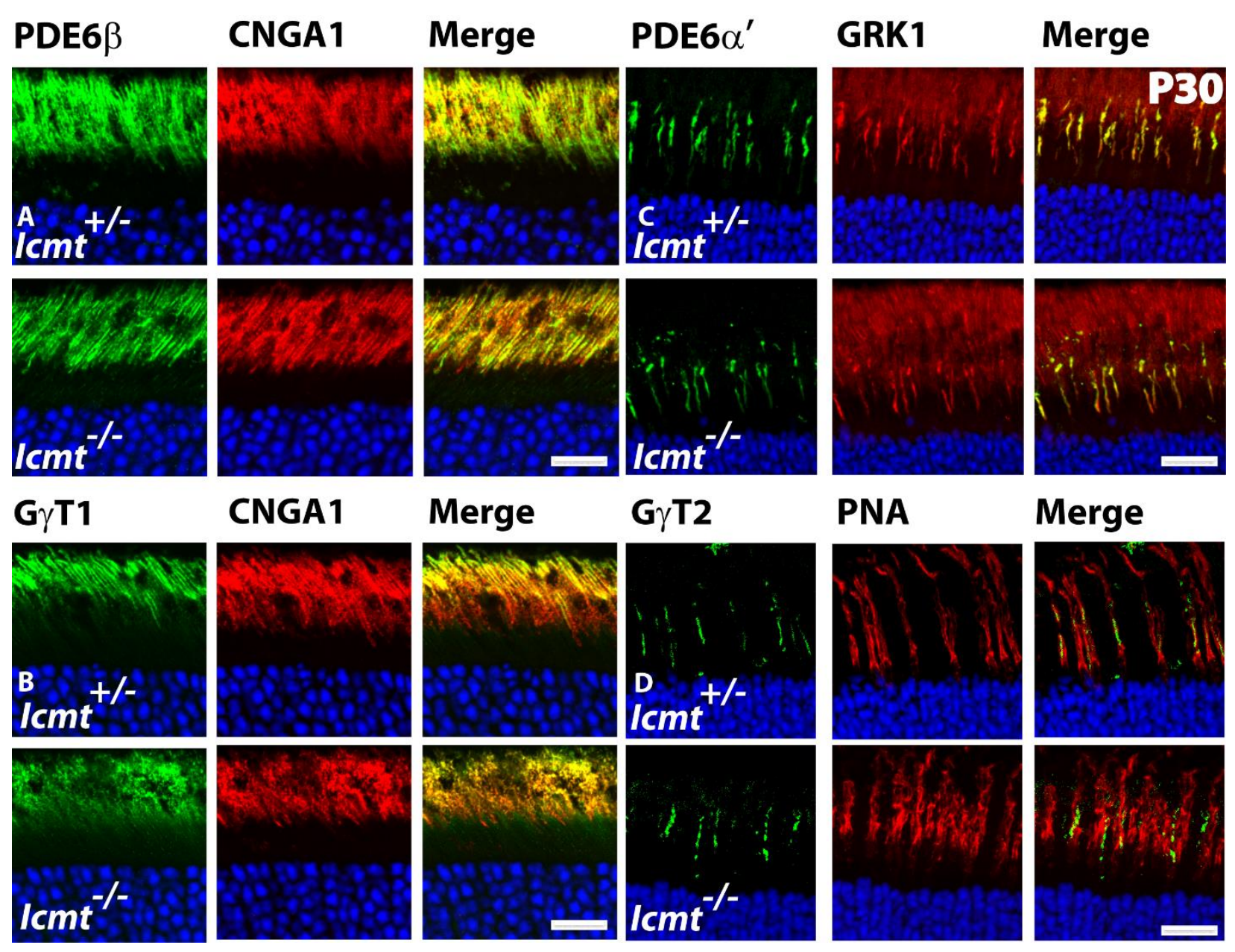

Figure 6 Rod PDE6 and transducin do not require ICMT-mediated protein methylation to be transported to the OS. 

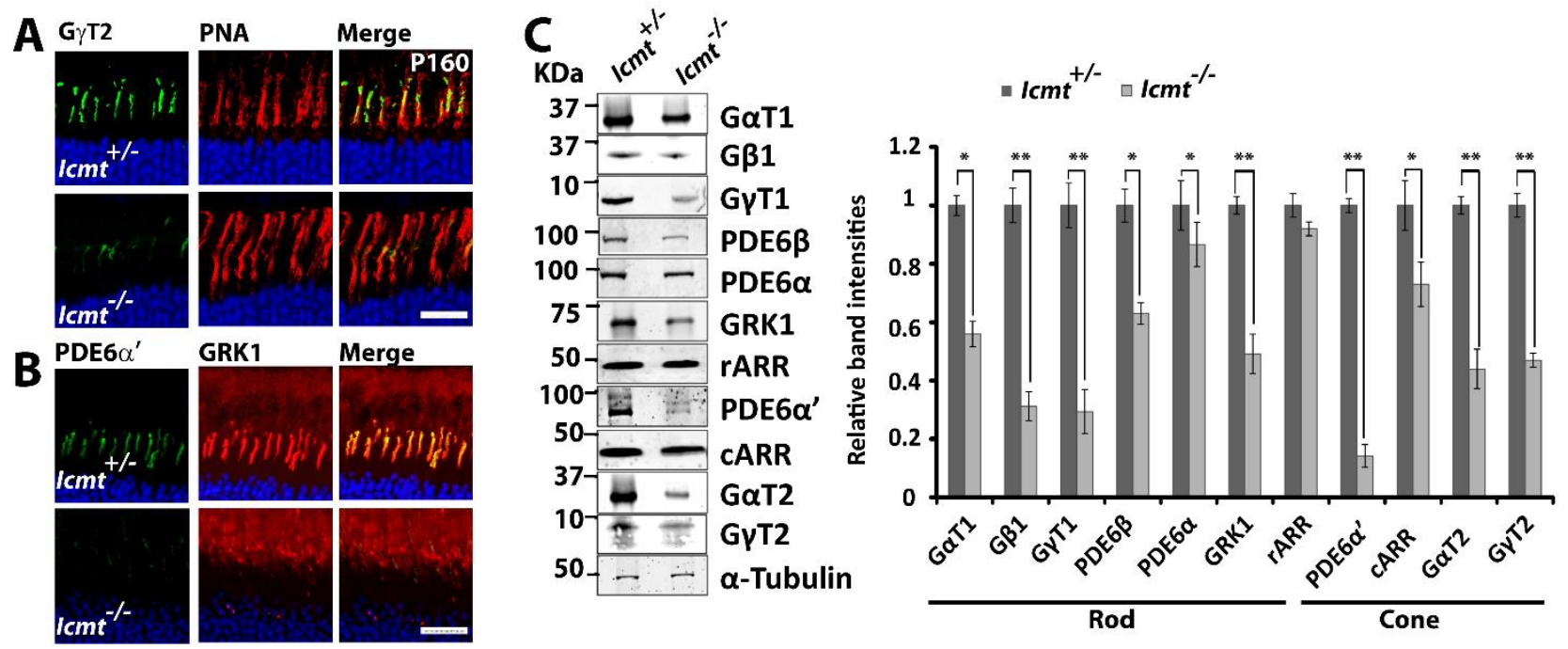

Figure 7 PDE6 and GRK1 levels in cones are reduced along with subset of other indicated proteins in mice lacking ICMT-mediated protein methylation. 


\section{CHAPTER 5}

\section{SUMMARY AND FUTURE DIRECTIONS}

Protein prenylation is a type of lipid protein modification that increases the hydrophobicity of proteins and is thought to play a role in membrane anchorage of proteins. Interestingly, even though multiple prenylated proteins are found in neurons, little is known about the need for them in these cell types. In particular, nothing is known about the importance of prenylation and post prenyl processing in regulating protein-protein interaction, assembly and their trafficking in retinal neurons. The purpose of my dissertation is to understand the role of prenylation and post prenyl processing in assembly, transport, membrane anchorage and the functions of key players of phototransduction cascade. Here, I have highlighted key findings from my work.

In the first study, we assessed the importance of prenylation in cone photoreceptor neurons that are essential for color vision (chapter 2). We generated mice, lacking Pggtlb (catalytic subunit of geranylgeranyl transferases) or Fntb (catalytic subunit of farnesyltransferases) in cone photoreceptor cells. We showed that the cone photoreceptors displayed a unique requirement of Pggt $1 \mathrm{~b}$ mediated geranylgeranylation for assembly of phosphodiesterase, the effector enzyme needed for phototransduction. It is important to note that the need for protein lipidation is specific for geranylgeranyl modification as cones can forgo the addition of farnesyl lipids to the proteins. Interestingly, absence of geranylgeranylation leads to complete loss of cone-mediated vision. The following are the most significant and novel findings from our analyses of this mouse model: 
1. Lipid modification of proteins is thought to be involved in only membrane anchoring. For the first time, we show that lipid modification is needed for assembly and stability of multimeric phosphodiesterase.

2. Our studies are the first to address the in vivo requirement for prenylation in neurons. Lack of Pggtlb mediated geranylgeranylation in the cone photoreceptors led to complete loss of cone mediated visual response. The requirement for geranylgeranylation was specific, as cones lacking farnesylation were not affected.

3. We demonstrate that the underlying cause of cone dysfunction is due to failure of chaperone AIPL1, a protein linked to childhood blindness, to interact with cone PDE6 leading to impaired assembly of functional cone PDE6. Absence of functional cone PDE6 in humans leads to color blindness.

4. Currently several research groups are using geranylgeranyl transferase inhibitors for treatment of cancer (157-159). Our studies show that such use is likely to affect human vision. Our studies show that caution should be exercised if these inhibitors were to cross bloodbrain barrier, as there is a risk of vision loss.

5. Multiple changes in PDE6 have been linked to visual disorders such as achromatopsia, cone dystrophy, RP and LCA. However, little is known about the mechanisms that operate behind these diseases. This is partly due to our lack of knowledge about synthesis, assembly and transport of PDE6 to ciliated outer segments. Our study provides a framework needed to understand this important process and is crucial in deciphering the mechanism behind these retinal degenerative diseases. 
Next we wanted to understand the effects of removal of prenyl transferases from entire retina, so we created a mice lacking prenyl transferases (chapter 3) in retina, using Cre-loxP recombination. Following are the key findings from this mouse model:

1. Here we show that, prenylation is crucial for survival and function of photoreceptor neurons (both rods and cones), as in the absence of Pggtlb or Fntb, the photoreceptor degenerate progressively. We believe one of the root cause behind age dependent degeneration is lack of firm membrane anchorage and reduced protein stability of PDE6 and transducin. Since FTases have multiple substrates such as INPP5E (149-152), Rab 8, Rab13, Rab 18 (160,161) and Rab 28 (91) that are present in inner segment, they are likely to be affected by removal of Fntb resulting in photoreceptor degeneration. We show that the inner segment protein, INPP5E is affected due to lack of farnesylation. Due to lack of good commercially available antibodies against Rab proteins, at this time we could not investigate more about Rab 8, Rab13, Rab 18 levels. Multiple disease such as rod and cone dystrophy, RP, age dependent retinal degeneration, LCA have been linked to the defects in PDE6, Rab proteins and transducin. Our studies suggest that lack of prenylation of PDE6 and transducin may be the root cause behind these visual defects. 2. In the mice lacking farnesyl transferases, we observed mislocalized rod transducin complex in the inner segment (IS) of photoreceptor under ambient light conditions. We strongly believe that this defect is due to lack of farnesylation of transducin $\gamma$ which is a known farnesylated protein $(36,74)$. This mislocalization of rod transducin may also contribute towards reduced ERG responses and photoreceptor degeneration.

3. More interestingly, we observed that the lack of farnesylation affects the transducin translocation kinetics. Efficient transducin translocation is needed for neuroprotection (to prevent damage to retinal neurons from sudden increase in light intensities) $(13,68,74,142)$. Our data 
suggests that the farnesylation of transducin $\gamma$ accelerates the return of translocated transducin from IS to OS. Defective translocation kinetics may also contribute to progressive photoreceptor degeneration in this mouse model.

4. Finally, we show that a single lipid anchor on multimeric rod PDE6 (either farnesyl anchor on PDE6 $\alpha$ or a geranylgeranyl anchor on PDE6 $\beta$ ) is sufficient for its assembly and transport to the OS. This was not the case with cone PDE6 where both PDE6 $\alpha$ ' subunits were thought to be geranylgeranylated, and the removal of GGTase-1 resulted in misassembly of cone PDE6 (chapter 2).

Previous studies demonstrated that RCE1-mediated proteolysis of prenylated proteins is necessary for photoreceptor maintenance and function (106). To understand the impact, the removal of ICMT has on retinal neurons, we created a mice lacking ICMT mediated methyl esterification in retinal neurons (59). Our study demonstrates the in-vivo requirement of ICMTmediated methylation of rod T $\gamma$ and PDE6 $\alpha$ ' for their membrane anchorage. Retinal development progressed normally as we observed no defects in the formation of retinal cell layers and photoreceptor function in mice lacking Icmt. The following are the most important findings from our studies of a targeted mouse knockout of Icmt:

1. There was an age-dependent decline in light-mediated rod and cone responses with a slight loss of photoreceptor neurons. At 5 months, photoresponses were reduced by $90 \%$ 2. We observed reductions in visual signal transduction protein levels, specifically rod transducin $\gamma$ and cone specific PDE6 $\alpha$ ' by P30.

3. Our study demonstrates the in-vivo requirement of ICMT- mediated methylation of rod Ty and PDE6 $\alpha$ ' for their membrane anchorage. In the absence of methylation, the majority of rod T $\gamma$ and PDE6 $\alpha$ ' does not associate with the membrane fraction. We believe that reduced 
membrane anchorage is the root cause of reduced protein stability and defective photoreceptormediated response.

4. Interestingly, in mice lacking Icmt, the residual rod T $\gamma$ and PDE6 $\alpha$ ' were transported to photoreceptor outer segment (OS). This finding is in contrast to our observations from retinas lacking Rce1, where PDE6 was mislocalized to photoreceptor inner segment (IS). This result suggests that the presence of the $-A A X$ at the C-termini of PDE6 catalytic subunits is detrimental for PDE6 transport to the OS.

Our results are the first in-vivo description of a targeted deletion of ICMT in retinal neurons.

\section{Future direction}

Although our present findings could highlight the complex role of prenyl transferases in photoreceptor neurons, there are a few questions that remain to be answered.

\section{Why cone PDE6 transport is unaffected in the absence of Pggt1b?}

We find that unassembled cone PDE6 is localized to cone OS in the absence of protein geranylgeranylation. We believe that N-terminal GAF domain of PDE6 bears a targeting motif

that aids in its localization to OS. To test this hypothesis, we can use transgenic mice model with mutations in GAF domain (that carries OS localization signal) and/or in $C A A X$ motif. This will help us understand the exact role of GAF domains and -CAAX motif in the localization.

\section{How does AIPL1 interact with cone PDE6 at molecular level?}

We show that the geranylgeranyl lipid anchors on cone PDE6 act as a "molecular grip" to facilitate the interaction of catalytic subunits of PDE6 with AIPL1 promoting its assembly, a step needed for synthesis of functional PDE6. At present, it is not clear if AIPL1 is cytosolic or ER 
membrane bound protein. The lipid anchors in cone PDE6 could potentially direct it to ER where AIPL1 may assist its folding and assembly. Further purification and crystallization studies will be needed to visualize the interactions between PDE6 and AIPL1. This will help us clarify the exact role of prenyl group in facilitating the interaction between cone PDE6 and AIPL1.

\section{What is the mechanism behind the role for AIPL1 as rod PDE6 chaperone?}

In the absence of AIPL1, PDE6 subunits do not assemble, function and are degraded. Lack of proper assembly and degradation is likely due to AIPL1's role as a chaperone. Does AIPL1 help in folding of $\alpha$ or $\beta$ subunit or both? What molecular regions in AIPL1 or PDE6 are involved in this interaction? Why does rod PDE6 need different lipid anchors if a single lipid anchor is sufficient for its assembly? This can be tested by making mutation in $C A A X$ sequence that will result in rod PDE6 subunits bearing same lipid group (either geranylgeranyl or farnesyl) or by removing both lipid anchors my mutating $C A A X$ sequence. The answers to some of these questions may help us predict the severity of particular AIPL1 mutation, associated disease progression and treatment potential.

\section{Is there a possibility of cross-prenylation?}

Since FTase and GGTase-I have overlapping substrates preferences, the possibility of cross prenylation cannot be ruled out. This can be tested using a prenyl transferase double knockout model in rods and cones.

\section{Is cone transducin- $\gamma(\mathrm{G} \gamma \mathrm{T} 2)$ geranylgeranylated?}

We observed altered membrane attachment and changes in Triton-X-114 partitioning of cone G-protein transducin (GүT2) in mice deficient in Pggtlb but not in Fntb animals. This 
finding was surprising as $\mathrm{G} \gamma \mathrm{T} 2$ is thought to be farnesylated based on its $C A A X$ sequence $(C V I S)$. Our experimental evidence suggests that $\mathrm{G} \gamma \mathrm{T} 2$ is a substrate of GGTase-I and is geranylgeranylated. In order to gain more clarity about the prenylation status, further investigation is needed. This will include series of experiments such as purification of G $\gamma \mathrm{T} 2$ from neural retina leucine zipper $(n r l)$ deficient mice (which will have only cone like photoreceptors) followed by mass spectrometry to identify the type of $C A A X$ modification on G $\gamma \mathrm{T} 2$.

\section{Do Rab proteins play a role in vesicular trafficking in cones?}

In our mouse model lacking Pggtlb, we showed the reduced stability of RetGC1, GRK1

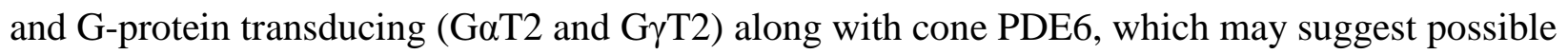
role of vesicular co-trafficking from IS to OS. We believe that Rab28, Rab18 or Rab13, which are present in inner segment are thought to be prenylated, (and/or) could be involved in the process of docking photoreceptor OS proteins on a vesicle. Generating antibodies specific to Rab28, Rab18 and Rab13 proteins could further test this hypothesis by performing immunoprecipitation assay to see protein-protein interaction, immunohistochemistry to confirm the localization of these Rab protein, prenyl transferase assays to check prenylation status. 


\section{REFERENCES}

1. Pearring, J. N., Salinas, R. Y., Baker, S. A., and Arshavsky, V. Y. (2013) Protein sorting, targeting and trafficking in photoreceptor cells. Progress in retinal and eye research 36, 24-51

2. Lamb, T. D., and Pugh, E. N. (2006) Phototransduction, dark adaptation, and rhodopsin regeneration the proctor lecture. Investigative ophthalmology \& visual science 47, 51385152

3. Young, R. W. (1967) The renewal of photoreceptor cell outer segments. The Journal of cell biology 33, 61-72

4. Oesterhelt, D., and Stoeckenius, W. (1973) Functions of a new photoreceptor membrane. Proceedings of the National Academy of Sciences 70, 2853-2857

5. Linton, J. D., Holzhausen, L. C., Babai, N., Song, H., Miyagishima, K. J., Stearns, G. W., Lindsay, K., Wei, J., Chertov, A. O., and Peters, T. A. (2010) Flow of energy in the outer retina in darkness and in light. Proceedings of the National Academy of Sciences 107, 8599-8604

6. Besharse, J. C., Hollyfield, J. G., and Rayborn, M. E. (1977) Turnover of rod photoreceptor outer segments. II. Membrane addition and loss in relationship to light. The Journal of cell biology 75, 507-527

7. Yamamoto, H., Simon, A., Eriksson, U., Harris, E., Berson, E. L., and Dryja, T. P. (1999) Mutations in the gene encoding 11-cis retinol dehydrogenase cause delayed dark adaptation and fundus albipunctatus. Nature genetics 22, 188-191

8. Schwartz, M. L., Hurley, J. B., and Ramamurthy, V. (2006) Biochemical Function of the LCA Linked Protien, Aryl Hydrocarbon Receptor Interacting Protein Like-1 (AIPL1). in Retinal Degenerative Diseases, Springer. pp 89-94

9. Ramamurthy, V., Roberts, M., van den Akker, F., Niemi, G., Reh, T., and Hurley, J. B. (2003) AIPL1, a protein implicated in Leber's congenital amaurosis, interacts with and aids in processing of farnesylated proteins. Proceedings of the National Academy of Sciences 100, 12630-12635

10. Muradov, H., Boyd, K. K., Haeri, M., Kerov, V., Knox, B. E., and Artemyev, N. O. (2009) Characterization of human cone phosphodiesterase-6 ectopically expressed in Xenopus laevis rods. Journal of Biological Chemistry 284, 32662-32669

11. Deretic, D. (2006) A role for rhodopsin in a signal transduction cascade that regulates membrane trafficking and photoreceptor polarity. Vision research 46, 4427-4433

12. Baehr, W. (2014) Membrane Protein Transport in Photoreceptors: The Function of PDE 6 The Proctor Lecture. Investigative ophthalmology \& visual science 55, 8653-8666

13. Calvert, P. D., Strissel, K. J., Schiesser, W. E., Pugh, E. N., and Arshavsky, V. Y. (2006) Light-driven translocation of signaling proteins in vertebrate photoreceptors. Trends in cell biology 16, 560-568

14. Hartman, H. L., Hicks, K. A., and Fierke, C. A. (2005) Peptide specificity of protein prenyltransferases is determined mainly by reactivity rather than binding affinity. Biochemistry 44, 15314-15324

15. Zhang, F. L., and Casey, P. J. (1996) Protein prenylation: molecular mechanisms and functional consequences. Annual review of biochemistry 65, 241-269 
16. Bergo, M. O., Lieu, H. D., Gavino, B. J., Ambroziak, P., Otto, J. C., Casey, P. J., Walker, Q. M., and Young, S. G. (2004) On the Physiological Importance of Endoproteolysis of CAAX Proteins HEART-SPECIFIC RCE1 KNOCKOUT MICE DEVELOP A LETHAL CARDIOMYOPATHY. Journal of Biological Chemistry 279, 4729-4736

17. Kim, E., Ambroziak, P., Otto, J. C., Taylor, B., Ashby, M., Shannon, K., Casey, P. J., and Young, S. G. (1999) Disruption of the mouse Rcel gene results in defective Ras processing and mislocalization of Ras within cells. J Biol Chem 274, 8383-8390

18. Wahlstrom, A. M., Cutts, B. A., Karlsson, C., Andersson, K. M., Liu, M., Sjogren, A. K., Swolin, B., Young, S. G., and Bergo, M. O. (2007) Rce1 deficiency accelerates the development of K-RAS-induced myeloproliferative disease. Blood 109, 763-768

19. Cox, A. D., and Der, C. J. (1992) Protein prenylation: more than just glue? Current opinion in cell biology 4, 1008-1016

20. Hrycyna, C., Bergo, M. O., and Tamanoi, F. (2011) Protein Prenylation, Academic Press

21. Roosing, S., Collin, R. W., den Hollander, A. I., Cremers, F. P., and Siemiatkowska, A. M. (2014) Prenylation defects in inherited retinal diseases. Journal of medical genetics 51, 143-151

22. Seabra, M. C., Ho, Y. K., and Anant, J. S. (1995) Deficient geranylgeranylation of Ram/Rab27 in choroideremia. Journal of Biological Chemistry 270, 24420-24427

23. Winter-Vann, A. M., and Casey, P. J. (2005) Post-prenylation-processing enzymes as new targets in oncogenesis. Nat Rev Cancer 5, 405-412

24. Magee, A. I., and Seabra, M. C. (2003) Are prenyl groups on proteins sticky fingers or greasy handles? Biochem J 376, e3-4

25. Kloog, Y., and Cox, A. D. (2004) Prenyl-binding domains: potential targets for Ras inhibitors and anti-cancer drugs. Semin Cancer Biol 14, 253-261

26. Norton, A. W., Hosier, S., Terew, J. M., Li, N., Dhingra, A., Vardi, N., Baehr, W., and Cote, R. H. (2005) Evaluation of the 17-kDa prenyl-binding protein as a regulatory protein for phototransduction in retinal photoreceptors. J Biol Chem 280, 1248-1256

27. Norton, A. W., Hosier, S., Terew, J. M., Li, N., Dhingra, A., Vardi, N., Baehr, W., and Cote, R. H. (2005) Evaluation of the 17-kDa prenyl-binding protein as a regulatory protein for phototransduction in retinal photoreceptors. Journal of Biological Chemistry 280, 1248-1256

28. Zhang, H., Hosier, S., Terew, J. M., Zhang, K., Cote, R. H., and Baehr, W. (2005) Assay and functional properties of PrBP(PDEdelta), a prenyl-binding protein interacting with multiple partners. Methods Enzymol 403, 42-56

29. Zhang, H., Li, S., Doan, T., Rieke, F., Detwiler, P., Frederick, J., and Baehr, W. (2007) Deletion of $\mathrm{PrBP} / \delta$ impedes transport of GRK1 and PDE6 catalytic subunits to photoreceptor outer segments. Proceedings of the National Academy of Sciences 104, 8857-8862

30. Zhang, H., Li, S., Doan, T., Rieke, F., Detwiler, P. B., Frederick, J. M., and Baehr, W. (2007) Deletion of PrBP/delta impedes transport of GRK1 and PDE6 catalytic subunits to photoreceptor outer segments. Proc Natl Acad Sci U S A 104, 8857-8862

31. Casey, P. J., and Seabra, M. C. (1996) Protein prenyltransferases. Journal of Biological Chemistry 271, 5289-5292

32. Magee, A. I., and Seabra, M. C. (2003) Are prenyl groups on proteins sticky fingers or greasy handles? Biochemical Journal 376, e3-e4 
33. Mijimolle, N., Velasco, J., Dubus, P., Guerra, C., Weinbaum, C. A., Casey, P. J., Campuzano, V., and Barbacid, M. (2005) Protein farnesyltransferase in embryogenesis, adult homeostasis, and tumor development. Cancer cell 7, 313-324

34. Sjogren, A.-K. M., Andersson, K. M., Liu, M., Cutts, B. A., Karlsson, C., Wahlstrom, A. M., Dalin, M., Weinbaum, C., Casey, P. J., and Tarkowski, A. (2007) GGTase-I deficiency reduces tumor formation and improves survival in mice with K-RAS-induced lung cancer. The Journal of clinical investigation 117, 1294-1304

35. Larijani, B., Hume, A. N., Tarafder, A. K., and Seabra, M. C. (2003) Multiple factors contribute to inefficient prenylation of Rab27a in Rab prenylation diseases. Journal of Biological Chemistry 278, 46798-46804

36. Fukada, Y., Takao, T., Ohguro, H., Yoshizawa, T., Akino, T., and Shimonishi, Y. (1990) Farnesylated gamma-subunit of photoreceptor G protein indispensable for GTP-binding. Nature 346, 658-660

37. Inglese, J., Koch, W. J., Caron, M. G., and Lefkowitz, R. J. (1992) Isoprenylation in regulation of signal transduction by G-protein-coupled receptor kinases. Nature 359, 147150

38. Anant, J. S., Ong, O. C., Xie, H. Y., Clarke, S., O'Brien, P. J., and Fung, B. K. (1992) In vivo differential prenylation of retinal cyclic GMP phosphodiesterase catalytic subunits. $J$ Biol Chem 267, 687-690

39. Ong, O. C., Ota, I. M., Clarke, S., and Fung, B. K. (1989) The membrane binding domain of rod cGMP phosphodiesterase is posttranslationally modified by methyl esterification at a C-terminal cysteine. Proc Natl Acad Sci U S A 86, 9238-9242

40. Pullarkat, R. K., and Morris, G. N. (1997) Farnesylation of Batten disease CLN3 protein. Neuropediatrics 28, 42-44

41. Gillespie, P. G., and Beavo, J. A. (1988) Characterization of a bovine cone photoreceptor phosphodiesterase purified by cyclic GMP-sepharose chromatography. Journal of Biological Chemistry 263, 8133-8141

42. Christiansen, J. R., Pendse, N. D., Kolandaivelu, S., Bergo, M. O., Young, S. G., and Ramamurthy, V. (2016) Deficiency of Isoprenylcysteine Carboxyl Methyltransferase (ICMT) Leads to Progressive Loss of Photoreceptor Function. J Neurosci 36, 5107-5114

43. Zhang, F. L., and Casey, P. J. (1996) Protein prenylation: molecular mechanisms and functional consequences. Annu Rev Biochem 65, 241-269

44. Bergo, M. O., Lieu, H. D., Gavino, B. J., Ambroziak, P., Otto, J. C., Casey, P. J., Walker, Q. M., and Young, S. G. (2004) On the physiological importance of endoproteolysis of CAAX proteins: heart-specific RCE1 knockout mice develop a lethal cardiomyopathy. $J$ Biol Chem 279, 4729-4736

45. Michaelson, D., Ali, W., Chiu, V. K., Bergo, M., Silletti, J., Wright, L., Young, S. G., and Philips, M. (2005) Postprenylation CAAX processing is required for proper localization of Ras but not Rho GTPases. Mol Biol Cell 16, 1606-1616

46. Christiansen, J. R., Kolandaivelu, S., Bergo, M. O., and Ramamurthy, V. (2011) RASconverting enzyme 1-mediated endoproteolysis is required for trafficking of rod phosphodiesterase 6 to photoreceptor outer segments. Proc Natl Acad Sci U S A

47. Hrycyna, C. A., Sapperstein, S. K., Clarke, S., and Michaelis, S. (1991) The Saccharomyces cerevisiae STE14 gene encodes a methyltransferase that mediates Cterminal methylation of a-factor and RAS proteins. EMBO J 10, 1699-1709 
48. Griggs, A. M., Hahne, K., and Hrycyna, C. A. (2010) Functional oligomerization of the Saccharomyces cerevisiae isoprenylcysteine carboxyl methyltransferase, Ste14p. J Biol Chem 285, 13380-13387

49. Anderson, J. L., and Hrycyna, C. A. (2006) 9 Structure and function of isoprenylcysteine carboxylmethyltransferase (Icmt): A key enzyme in CaaX processing. The Enzymes 24, 245-272

50. Swanson, R. J., and Applebury, M. L. (1983) Methylation of proteins in photoreceptor rod outer segments. J Biol Chem 258, 10599-10605

51. Ohguro, H., Fukada, Y., and Akino, T. (1991) Structure and function of gamma-subunit of photoreceptor G-protein (transducin). Comp Biochem Physiol B 100, 433-438

52. Fukada, Y., Takao, T., Ohguro, H., Yoshizawa, T., Akino, T., and Shimonishi, Y. (1990) Farnesylated gamma-subunit of photoreceptor G protein indispensable for GTP-binding. Nature 346, 658-660

53. Bergo, M. O., Leung, G. K., Ambroziak, P., Otto, J. C., Casey, P. J., Gomes, A. Q., Seabra, M. C., and Young, S. G. (2001) Isoprenylcysteine carboxyl methyltransferase deficiency in mice. J Biol Chem 276, 5841-5845

54. Svensson, A. W., Casey, P. J., Young, S. G., and Bergo, M. O. (2006) Genetic and pharmacologic analyses of the role of Icmt in ras membrane association and function. Methods in enzymology 407, 144-159

55. Bergo, M. O., Gavino, B. J., Hong, C., Beigneux, A. P., McMahon, M., Casey, P. J., and Young, S. G. (2004) Inactivation of Icmt inhibits transformation by oncogenic K-Ras and B-Raf. J Clin Invest 113, 539-550

56. Parish, C. A., and Rando, R. R. (1996) Isoprenylation/methylation of proteins enhances membrane association by a hydrophobic mechanism. Biochemistry 35, 8473-8477

57. Cook, T. A., Ghomashchi, F., Gelb, M. H., Florio, S. K., and Beavo, J. A. (2000) Binding of the delta subunit to rod phosphodiesterase catalytic subunits requires methylated, prenylated C-termini of the catalytic subunits. Biochemistry 39, 13516-13523

58. Ohguro, H., Fukada, Y., Takao, T., Shimonishi, Y., Yoshizawa, T., and Akino, T. (1991) Carboxyl methylation and farnesylation of transducin gamma-subunit synergistically enhance its coupling with metarhodopsin II. The EMBO journal 10, 3669

59. Christiansen, J. R., Pendse, N. D., Kolandaivelu, S., Bergo, M. O., Young, S. G., and Ramamurthy, V. (2016) Deficiency of Isoprenylcysteine Carboxyl Methyltransferase (ICMT) Leads to Progressive Loss of Photoreceptor Function. The Journal of Neuroscience 36, 5107-5114

60. Linton, J. D., Holzhausen, L. C., Babai, N., Song, H., Miyagishima, K. J., Stearns, G. W., Lindsay, K., Wei, J., Chertov, A. O., Peters, T. A., Caffe, R., Pluk, H., Seeliger, M. W., Tanimoto, N., Fong, K., Bolton, L., Kuok, D. L., Sweet, I. R., Bartoletti, T. M., Radu, R. A., Travis, G. H., Zagotta, W. N., Townes-Anderson, E., Parker, E., Van der Zee, C. E., Sampath, A. P., Sokolov, M., Thoreson, W. B., and Hurley, J. B. (2010) Flow of energy in the outer retina in darkness and in light. Proc Natl Acad Sci U S A 107, 8599-8604

61. Young, R. W. (1967) The renewal of photoreceptor cell outer segments. J Cell Biol 33, 61-72

62. Liu, Q., Tan, G., Levenkova, N., Li, T., Pugh, E. N., Jr., Rux, J. J., Speicher, D. W., and Pierce, E. A. (2007) The proteome of the mouse photoreceptor sensory cilium complex. Mol Cell Proteomics 6, 1299-1317 
63. Artemyev, N. O., Natochin, M., Busman, M., Schey, K. L., and Hamm, H. E. (1996) Mechanism of photoreceptor cGMP phosphodiesterase inhibition by its gamma-subunits. Proceedings of the National Academy of Sciences 93, 5407-5412

64. Muradov, K. G., Granovsky, A. E., Schey, K. L., and Artemyev, N. O. (2002) Direct interaction of the inhibitory $\gamma$-subunit of rod cGMP phosphodiesterase (PDE6) with the PDE6 GAFa domains. Biochemistry 41, 3884-3890

65. Tcheudji, J. F. K., Lebeau, L., Virmaux, N., Maftei, C. G., Cote, R. H., Lugnier, C., and Schultz, P. (2001) Molecular organization of bovine rod cGMP-phosphodiesterase 6. Journal of molecular biology 310, 781-791

66. Burns, M. E., and Arshavsky, V. Y. (2005) Beyond counting photons: trials and trends in vertebrate visual transduction. Neuron 48, 387-401

67. Angleson, J. K., and Wensel, T. G. (1993) A GTPase-accelerating factor for transducin, distinct from its effector cGMP phosphodiesterase, in rod outer segment membranes. Neuron 11, 939-949

68. Lobanova, E. S., Finkelstein, S., Song, H., Tsang, S. H., Chen, C.-K., Sokolov, M., Skiba, N. P., and Arshavsky, V. Y. (2007) Transducin translocation in rods is triggered by saturation of the GTPase-activating complex. The Journal of neuroscience 27, 11511160

69. Matsuda, T., Takao, T., Shimonishi, Y., Murata, M., Asano, T., Yoshizawa, T., and Fukada, Y. (1994) Characterization of interactions between transducin alpha/beta gamma-subunits and lipid membranes. Journal of Biological Chemistry 269, 3035830363

70. Skiba, N. P., Hopp, J. A., and Arshavsky, V. Y. (2000) The effector enzyme regulates the duration of $\mathrm{G}$ protein signaling in vertebrate photoreceptors by increasing the affinity between transducin and RGS protein. Journal of Biological Chemistry 275, 32716-32720

71. Kerov, V., Rubin, W. W., Natochin, M., Melling, N. A., Burns, M. E., and Artemyev, N. O. (2007) N-terminal fatty acylation of transducin profoundly influences its localization and the kinetics of photoresponse in rods. J Neurosci 27, 10270-10277

72. Kolesnikov, A. V., Rikimaru, L., Hennig, A. K., Lukasiewicz, P. D., Fliesler, S. J., Govardovskii, V. I., Kefalov, V. J., and Kisselev, O. G. (2011) G-protein betagammacomplex is crucial for efficient signal amplification in vision. $J$ Neurosci 31, 8067-8077

73. Michaelson, D., Ahearn, I., Bergo, M., Young, S., and Philips, M. (2002) Membrane trafficking of heterotrimeric $\mathrm{G}$ proteins via the endoplasmic reticulum and Golgi. Mol Biol Cell 13, 3294-3302

74. Kassai, H., Aiba, A., Nakao, K., Nakamura, K., Katsuki, M., Xiong, W.-H., Yau, K.-W., Imai, H., Shichida, Y., and Satomi, Y. (2005) Farnesylation of retinal transducin underlies its translocation during light adaptation. Neuron 47, 529-539

75. Finkelstein, S., Lobanova, E., and Arshavsky, V. (2009) Knockout of the Rod Transducin Gamma Subunit Induces Cell Death. Investigative Ophthalmology \& Visual Science 50, 4472-4472

76. Miyake, Y., Yagasaki, K., Horiguchi, M., Kawase, Y., and Kanda, T. (1986) Congenital stationary night blindness with negative electroretinogram: a new classification. Archives of ophthalmology 104, 1013-1020

77. Sandberg, M. A., Pawlyk, B. S., Dan, J., Arnaud, B., Dryja, T. P., and Berson, E. L. (1998) Rod and cone function in the Nougaret form of stationary night blindness. Archives of Ophthalmology 116, 867-872 
78. Baehr, W., Devlin, M. J., and Applebury, M. L. (1979) Isolation and characterization of cGMP phosphodiesterase from bovine rod outer segments. J Biol Chem 254, 1166911677

79. Gillespie, P. G., Prusti, R. K., Apel, E. D., and Beavo, J. A. (1989) A soluble form of bovine rod photoreceptor phosphodiesterase has a novel $15-\mathrm{kDa}$ subunit. J Biol Chem 264, 12187-12193

80. Cote, R. H. (2006) Photoreceptor phosphodiesterase (PDE6): a G-protein-activated PDE regulating visual excitation in rod and cone photoreceptor cells.

81. Veske, A., Orth, U., Ruther, K., Zrenner, E., Rosenberg, T., Baehr, W., Gal, A. (1995) Mutations in the gene fro the b-subunit of rod photoreceptor cgmp-specific phosphodiesterase (PDEB) in patients with retinal dystrophies and dysfunctions. Degenerative Diseases of the Retina, 313-322

82. Kolandaivelu, S., Chang, B., and Ramamurthy, V. (2011) Rod Phosphodiesterase-6 (PDE6) catalytic subunts restores cone function in a mouse model lacking cone PDE6 catalytic subunit. J Biol Chem

83. Ramamurthy, V., Niemi, G. A., Reh, T. A., and Hurley, J. B. (2004) Leber congenital amaurosis linked to AIPL1: a mouse model reveals destabilization of cGMP phosphodiesterase. Proceedings of the National Academy of Sciences of the United States of America 101, 13897-13902

84. Kolandaivelu, S., Huang, J., Hurley, J. B., and Ramamurthy, V. (2009) AIPL1, a protein associated with childhood blindness, interacts with $\alpha$-subunit of rod phosphodiesterase (PDE6) and is essential for its proper assembly. Journal of Biological Chemistry 284, 30853-30861

85. Kolandaivelu, S., and Ramamurthy, V. (2014) AIPL1 protein and its indispensable role in cone photoreceptor function and survival. in Retinal Degenerative Diseases, Springer. pp 43-48

86. Kolandaivelu, S., Singh, R. K., and Ramamurthy, V. (2013) AIPL1, A protein linked to blindness, is essential for the stability of enzymes mediating cGMP metabolism in cone photoreceptor cells. Human molecular genetics, ddt496

87. Kirschman, L. T., Kolandaivelu, S., Frederick, J. M., Dang, L., Goldberg, A. F., Baehr, W., and Ramamurthy, V. (2009) The Leber congenital amaurosis protein, AIPL1, is needed for the viability and functioning of cone photoreceptor cells. Human molecular genetics, ddp571

88. Horner, T. J., Osawa, S., Schaller, M. D., and Weiss, E. R. (2005) Phosphorylation of GRK1 and GRK7 by cAMP-dependent protein kinase attenuates their enzymatic activities. Journal of Biological Chemistry 280, 28241-28250

89. Weiss, E. R., Ducceschi, M. H., Horner, T. J., Li, A., Craft, C. M., and Osawa, S. (2001) Species-specific differences in expression of G-protein-coupled receptor kinase (GRK) 7 and GRK1 in mammalian cone photoreceptor cells: implications for cone cell phototransduction. The Journal of Neuroscience 21, 9175-9184

90. Chen, C. K., Burns, M. E., Spencer, M., Niemi, G. A., Chen, J., Hurley, J. B., Baylor, D. A., and Simon, M. I. (1999) Abnormal photoresponses and light-induced apoptosis in rods lacking rhodopsin kinase. Proc Natl Acad Sci U S A 96, 3718-3722

91. Roosing, S., Rohrschneider, K., Beryozkin, A., Sharon, D., Weisschuh, N., Staller, J., Kohl, S., Zelinger, L., Peters, T. A., and Neveling, K. (2013) Mutations in RAB28, 
encoding a farnesylated small GTPase, are associated with autosomal-recessive cone-rod dystrophy. The American Journal of Human Genetics 93, 110-117

92. Roosing, S., Thiadens, A. A., Hoyng, C. B., Klaver, C. C., den Hollander, A. I., and Cremers, F. P. (2014) Causes and consequences of inherited cone disorders. Progress in retinal and eye research $\mathbf{4 2}, 1-26$

93. Ávila-Fernández, A., Cantalapiedra, D., Aller, E., Vallespín, E., Aguirre-Lambán, J., Blanco-Kelly, F., Corton, M., Riveiro-Álvarez, R., Allikmets, R., and Trujillo-Tiebas, M. J. (2010) Mutation analysis of 272 Spanish families affected by autosomal recessive retinitis pigmentosa using a genotyping microarray.

94. Armstrong, S. A., Brown, M. S., Goldstein, J. L., and Seabra, M. C. (1995) [5] Preparation of recombinant Rab geranylgeranyltransferase and Rab escort proteins. Methods in enzymology 257, 30-41

95. Pereira-Leal, J. B., Hume, A. N., and Seabra, M. C. (2001) Prenylation of Rab GTPases: molecular mechanisms and involvement in genetic disease. FEBS letters 498, 197-200

96. Lim, Y. S., Chua, C. E. L., and Tang, B. L. (2011) Rabs and other small GTPases in ciliary transport. Biology of the Cell 103, 209-221

97. Sanders, A. A. W. M. (2014) Identification and functional characterisation of new ciliary base proteins and investigation of diffusion kinetics across the ciliary transition zone in Caenorhabditis elegans roundworms.

98. Järvelä, I., Lehtovirta, M., Tikkanen, R., Kyttälä, A., and Jalanko, A. (1999) Defective intracellular transport of CLN3 is the molecular basis of Batten disease (JNCL). Human molecular genetics 8, 1091-1098

99. Katz, M. L., Gao, C.-L., Prabhakaram, M., Shibuya, H., Liu, P.-C., and Johnson, G. S. (1997) Immunochemical localization of the Batten disease (CLN3) protein in retina. Investigative ophthalmology \& visual science 38, 2375-2386

100. Lerner, T. J., Boustany, R.-M. N., Anderson, J. W., D'Arigo, K. L., Schlumpf, K., Buckler, A. J., Gusella, J. F., and Haines, J. L. (1995) Isolation of a novel gene underlying Batten disease, CLN3. Cell 82, 949-957

101. Seigel, G. M., Lotery, A., Kummer, A., Bernard, D. J., Greene, N. D., Turmaine, M., Derksen, T., Nussbaum, R. L., Davidson, B., and Wagner, J. (2002) Retinal pathology and function in a Cln3 knockout mouse model of juvenile Neuronal Ceroid Lipofuscinosis (batten disease). Molecular and Cellular Neuroscience 19, 515-527

102. Luiro, K., Kopra, O., Lehtovirta, M., and Jalanko, A. (2001) CLN3 protein is targeted to neuronal synapses but excluded from synaptic vesicles: new clues to Batten disease. Human molecular genetics 10, 2123-2131

103. Pereira-Leal, J. B., Hume, A. N., and Seabra, M. C. (2001) Prenylation of Rab GTPases: molecular mechanisms and involvement in genetic disease. FEBS Lett 498, 197-200

104. Petersen-Jones, S. M., Entz, D. D., and Sargan, D. R. (1999) cGMP phosphodiesterasealpha mutation causes progressive retinal atrophy in the Cardigan Welsh corgi dog. Invest Ophthalmol Vis Sci 40, 1637-1644

105. Suber, M. L., Pittler, S. J., Qin, N., Wright, G. C., Holcombe, V., Lee, R. H., Craft, C. M., Lolley, R. N., Baehr, W., and Hurwitz, R. L. (1993) Irish setter dogs affected with $\mathrm{rod} /$ cone dysplasia contain a nonsense mutation in the rod cGMP phosphodiesterase betasubunit gene. Proc Natl Acad Sci U S A 90, 3968-3972

106. Christiansen, J. R., Kolandaivelu, S., Bergo, M. O., and Ramamurthy, V. (2011) RASconverting enzyme 1-mediated endoproteolysis is required for trafficking of rod 
phosphodiesterase 6 to photoreceptor outer segments. Proceedings of the National Academy of Sciences 108, 8862-8866

107. Qin, N., and Baehr, W. (1994) Expression and mutagenesis of mouse rod photoreceptor cGMP phosphodiesterase. Journal of Biological Chemistry 269, 3265-3271

108. Ong, O. C., Ota, I. M., Clarke, S., and Fung, B. (1989) The membrane binding domain of rod cGMP phosphodiesterase is posttranslationally modified by methyl esterification at a C-terminal cysteine. Proceedings of the National Academy of Sciences 86, 9238-9242

109. Anant, J., Ong, O., Xie, H., Clarke, S., O'Brien, P., and Fung, B. (1992) In vivo differential prenylation of retinal cyclic GMP phosphodiesterase catalytic subunits. Journal of Biological Chemistry 267, 687-690

110. Inglese, J., Koch, W. J., Caron, M. G., and Lefkowitz, R. J. (1992) Isoprenylation in regulation of signal transduction by G-protein-coupled receptor kinases. Nature 359, 147150

111. Akhmedov, N. B., Piriev, N. I., Pearce-Kelling, S., Acland, G. M., Aguirre, G. D., and Farber, D. B. (1998) Canine cone transducin-gamma gene and cone degeneration in the cd dog. Investigative ophthalmology \& visual science 39, 1775-1781

112. Bigay, J., Faurobert, E., Franco, M., and Chabre, M. (1994) Roles of lipid modifications of transducin subunits in their GDP-dependent association and membrane binding. Biochemistry 33, 14081-14090

113. Wensel, T. G. (2008) Signal transducing membrane complexes of photoreceptor outer segments. Vision research 48, 2052-2061

114. Cote, R. (2004) Characteristics of photoreceptor PDE (PDE6): similarities and differences to PDE5. International journal of impotence research 16, S28-S33

115. Li, T., Volpp, K., and Applebury, M. L. (1990) Bovine cone photoreceptor cGMP phosphodiesterase structure deduced from a cDNA clone. Proceedings of the National Academy of Sciences 87, 293-297

116. Baehr, W., Devlin, M. J., and Applebury, M. L. (1979) Isolation and characterization of cGMP phosphodiesterase from bovine rod outer segments. J Biol Chem 254, 1166911677

117. Deterre, P., Bigay, J., Forquet, F., Robert, M., and Chabre, M. (1988) cGMP phosphodiesterase of retinal rods is regulated by two inhibitory subunits. Proceedings of the National Academy of Sciences 85, 2424-2428

118. Hamilton, S. E., and Hurley, J. B. (1990) A phosphodiesterase inhibitor specific to a subset of bovine retinal cones. Journal of Biological Chemistry 265, 11259-11264

119. Zhang, Z., He, F., Constantine, R., Baker, M. L., Baehr, W., Schmid, M. F., Wensel, T. G., and Agosto, M. A. (2015) Domain organization and conformational plasticity of the $\mathrm{G}$ protein effector, PDE6. Journal of Biological Chemistry 290, 12833-12843

120. Kolandaivelu, S., Chang, B., and Ramamurthy, V. (2011) Rod phosphodiesterase-6 (PDE6) catalytic subunits restore cone function in a mouse model lacking cone PDE6 catalytic subunit. Journal of Biological Chemistry 286, 33252-33259

121. Le, Y.-Z., Ash, J. D., Al-Ubaidi, M. R., Chen, Y., Ma, J.-X., and Anderson, R. E. (2004) Targeted expression of Cre recombinase to cone photoreceptors in transgenic mice. $\mathrm{Mol}$ Vis 10, 1011-1018

122. Justice, J. M., Murtagh, J. J., Moss, J., and Vaughan, M. (1995) Hydrophobicity and subunit interactions of rod outer segment proteins investigated using Triton X-114 phase partitioning. Journal of Biological Chemistry 270, 17970-17976 
123. Hurwitz, R. L., Bunt-Milam, A. H., and Beavo, J. A. (1984) Immunologic characterization of the photoreceptor outer segment cyclic GMP phosphodiesterase. Journal of Biological Chemistry 259, 8612-8618

124. Cheguru, P., Zhang, Z., and Artemyev, N. O. (2014) The GAFa domain of phosphodiesterase- 6 contains a rod outer segment localization signal. Journal of neurochemistry 129, 256-263

125. Muradov, H., Boyd, K. K., and Artemyev, N. O. (2010) Rod phosphodiesterase-6 PDE6A and PDE6B subunits are enzymatically equivalent. Journal of Biological Chemistry 285, 39828-39834

126. Baker, S. A., Haeri, M., Yoo, P., Gospe, S. M., Skiba, N. P., Knox, B. E., and Arshavsky, V. Y. (2008) The outer segment serves as a default destination for the trafficking of membrane proteins in photoreceptors. The Journal of cell biology 183, 485-498

127. Santucci, R., Mackley, P. A., Sebti, S., and Alsina, M. (2003) Farnesyltransferase inhibitors and their role in the treatment of multiple myeloma. Cancer Control 10, 384387

128. Karan, S., Zhang, H., Li, S., Frederick, J. M., and Baehr, W. (2008) A model for transport of membrane-associated phototransduction polypeptides in rod and cone photoreceptor inner segments. Vision research 48, 442-452

129. Apolloni, A., Prior, I. A., Lindsay, M., Parton, R. G., and Hancock, J. F. (2000) H-ras but not K-ras traffics to the plasma membrane through the exocytic pathway. Molecular and cellular biology 20, 2475-2487

130. Choy, E., Chiu, V. K., Silletti, J., Feoktistov, M., Morimoto, T., Michaelson, D., Ivanov, I. E., and Philips, M. R. (1999) Endomembrane trafficking of ras: the CAAX motif targets proteins to the ER and Golgi. Cell 98, 69-80

131. Seabra, M. C. (1998) Membrane association and targeting of prenylated Ras-like GTPases. Cellular signalling 10, 167-172

132. Lee, R., Chang, S. Y., Trinh, H., Tu, Y., White, A. C., Davies, B. S., Bergo, M. O., Fong, L. G., Lowry, W. E., and Young, S. G. (2010) Genetic studies on the functional relevance of the protein prenyltransferases in skin keratinocytes. Human molecular genetics, ddq036

133. Khan, O. M., Ibrahim, M. X., Jonsson, M., Karlsson, C., Liu, M., Sjogren, A.-K. M., Olofsson, F. J., Brisslert, M., Andersson, S., and Ohlsson, C. (2011)

Geranylgeranyltransferase type I (GGTase-I) deficiency hyperactivates macrophages and induces erosive arthritis in mice. The Journal of clinical investigation 121, 628-639

134. Beigneux, A., Withycombe, S. K., Digits, J. A., Tschantz, W. R., Weinbaum, C. A., Griffey, S. M., Bergo, M., Casey, P. J., and Young, S. G. (2002) Prenylcysteine lyase deficiency in mice results in the accumulation of farnesylcysteine and geranylgeranylcysteine in brain and liver. Journal of Biological Chemistry 277, 3835838363

135. Yang, S. H., Bergo, M. O., Farber, E., Qiao, X., Fong, L. G., and Young, S. G. (2009) Caution! Analyze transcripts from conditional knockout alleles. Transgenic research $\mathbf{1 8}$, 483-489

136. Yang, S. H., Meta, M., Qiao, X., Frost, D., Bauch, J., Coffinier, C., Majumdar, S., Bergo, M. O., Young, S. G., and Fong, L. G. (2006) A farnesyltransferase inhibitor improves disease phenotypes in mice with a Hutchinson-Gilford progeria syndrome mutation. The Journal of clinical investigation 116, 2115-2121 
137. Goc, A., Chami, M., Lodowski, D. T., Bosshart, P., Moiseenkova-Bell, V., Baehr, W., Engel, A., and Palczewski, K. (2010) Structural characterization of the rod cGMP phosphodiesterase 6. Journal of molecular biology 401, 363-373

138. Wensel, T. G., and Stryer, L. (1986) Reciprocal control of retinal rod cyclic GMP phosphodiesterase by its $\gamma$ subunit and transducin. Proteins: Structure, Function, and Bioinformatics 1, 90-99

139. Kokame, K., Fukada, Y., Yoshizawa, T., Takao, T., and Shimonishi, Y. (1992) Lipid modification at the $\mathrm{N}$ terminus of photoreceptor G-protein $\alpha$-subunit.

140. Linder, M. E., Middleton, P., Hepler, J. R., Taussig, R., Gilman, A. G., and Mumby, S. M. (1993) Lipid modifications of $G$ proteins: alpha subunits are palmitoylated.

Proceedings of the National Academy of Sciences 90, 3675-3679

141. Wedegaertner, P. B., Wilson, P. T., and Bourne, H. R. (1995) Lipid modifications of trimeric $\mathrm{G}$ proteins. Journal of Biological Chemistry 270, 503-506

142. Sokolov, M., Lyubarsky, A. L., Strissel, K. J., Savchenko, A. B., Govardovskii, V. I., Pugh, E. N., and Arshavsky, V. Y. (2002) Massive light-driven translocation of transducin between the two major compartments of rod cells: a novel mechanism of light adaptation. Neuron 34, 95-106

143. Sokolov, M., Strissel, K. J., Leskov, I. B., Michaud, N. A., Govardovskii, V. I., and Arshavsky, V. Y. (2004) Phosducin Facilitates Light-driven Transducin Translocation in Rod Photoreceptors EVIDENCE FROM THE PHOSDUCIN KNOCKOUT MOUSE. Journal of Biological Chemistry 279, 19149-19156

144. Pittler, S. J., Fliesler, S. J., Fisher, P. L., Keller, P., and Rapp, L. M. (1995) In vivo requirement of protein prenylation for maintenance of retinal cytoarchitecture and photoreceptor structure. The Journal of cell biology 130, 431-439

145. Furuta, Y., Lagutin, O., Hogan, B. L., and Oliver, G. C. (2000) Retina-and ventral forebrain-specific Cre recombinase activity in transgenic mice. Genesis 26, 130-132

146. Liu, M., Sjogren, A.-K. M., Karlsson, C., Ibrahim, M. X., Andersson, K. M., Olofsson, F. J., Wahlstrom, A. M., Dalin, M., Yu, H., and Chen, Z. (2010) Targeting the protein prenyltransferases efficiently reduces tumor development in mice with K-RAS-induced lung cancer. Proceedings of the National Academy of Sciences 107, 6471-6476

147. Rattner, A., Sun, H., and Nathans, J. (1999) Molecular genetics of human retinal disease. Annual review of genetics 33, 89-131

148. Hartong, D. T., Berson, E. L., and Dryja, T. P. (2006) Retinitis pigmentosa. The Lancet 368, 1795-1809

149. Saada, J., Perrault, I., Amiel, J., Litzler, J., Filhol, E., Elkhartoufi, N., Kwong, M., Burglen, L., Chassaing, N., and é Encha-Ravazi, F. A Homozygous PDE6D Mutation in Joubert Syndrome Impairs Targeting of Farnesylated INPP5E Protein to the Primary Cilium.

150. Bielas, S. L., Silhavy, J. L., Brancati, F., Kisseleva, M. V., Al-Gazali, L., Sztriha, L., Bayoumi, R. A., Zaki, M. S., Abdel-Aleem, A., and Rosti, R. O. (2009) Mutations in INPP5E, encoding inositol polyphosphate-5-phosphatase E, link phosphatidyl inositol signaling to the ciliopathies. Nature genetics 41, 1032-1036

151. Jacoby, M., Cox, J. J., Gayral, S., Hampshire, D. J., Ayub, M., Blockmans, M., Pernot, E., Kisseleva, M. V., Compère, P., and Schiffmann, S. N. (2009) INPP5E mutations cause primary cilium signaling defects, ciliary instability and ciliopathies in human and mouse. Nature genetics 41, 1027-1031 
152. Rao, K. N., Zhang, W., Li, L., Anand, M., and Khanna, H. (2016) Prenylated retinal ciliopathy protein RPGR interacts with PDE6 $\delta$ and regulates ciliary localization of Joubert syndrome-associated protein INPP5E. Human Molecular Genetics, ddw281

153. Constantine, R., Zhang, H., Gerstner, C. D., Frederick, J. M., and Baehr, W. (2012) Uncoordinated (UNC) 119: coordinating the trafficking of myristoylated proteins. Vision research 75, 26-32

154. Zhang, H., Constantine, R., Vorobiev, S., Chen, Y., Seetharaman, J., Huang, Y. J., Xiao, R., Montelione, G. T., Gerstner, C. D., and Davis, M. W. (2011) UNC119 is required for $\mathrm{G}$ protein trafficking in sensory neurons. Nature neuroscience 14, 874-880

155. Furuta, Y., Lagutin, O., Hogan, B. L., and Oliver, G. C. (2000) Retina- and ventral forebrain-specific Cre recombinase activity in transgenic mice. Genesis 26, 130-132

156. Young SG, A. P., Kim E, Clarke S. (2000) Postisoprenylation protein processing: CXXX (CaaX) endoproteases and isoprenylcysteine carboxyl methyltransferase. The Enzymes, $155-213$

157. Kloog, Y., and Cox, A. D. (2004) Prenyl-binding domains: potential targets for Ras inhibitors and anti-cancer drugs. in Seminars in cancer biology, Elsevier

158. Lackner, M. R., Kindt, R. M., Carroll, P. M., Brown, K., Cancilla, M. R., Chen, C., de Silva, H., Franke, Y., Guan, B., and Heuer, T. (2005) Chemical genetics identifies Rab geranylgeranyl transferase as an apoptotic target of farnesyl transferase inhibitors. Cancer cell 7, 325-336

159. Sebti, S. M., and Hamilton, A. D. (2000) Farnesyltransferase and geranylgeranyltransferase I inhibitors and cancer therapy: lessons from mechanism and bench-to-bedside translational studies. Oncogene 19

160. Joberty, G., Tavitian, A., and Zahraoui, A. (1993) Isoprenylation of Rab proteins possessing a C-terminal Caax Motif. FEBS letters 330, 323-328

161. Leung, K. F., Baron, R., Ali, B. R., Magee, A. I., and Seabra, M. C. (2007) Rab GTPases containing a CAAX motif are processed post-geranylgeranylation by proteolysis and methylation. Journal of Biological Chemistry 282, 1487-1497 